\title{
Eigenfunction Expansions of Functions Describing Systems with Symmetries
}

\author{
Ivan KACHURYK ${ }^{\dagger}$ and Anatoliy KLIMYK ${ }^{\ddagger}$ \\ † Khmel'nyts'kyy National University, Khmel'nyts'kyy, Ukraine \\ E-mail: kachuryk@ief.tup.km.ua \\ $\ddagger$ Bogolyubov Institute for Theoretical Physics, 14-b Metrologichna Str., Kyiv-143, 03143 Ukraine \\ E-mail: aklimyk@bitp.kiev.ua
}

Received March 02, 2007; Published online March 28, 2007

Original article is available at http://www.emis.de/journals/SIGMA/2007/055/

\begin{abstract}
Physical systems with symmetries are described by functions containing kinematical and dynamical parts. We consider the case when kinematical symmetries are described by a noncompact semisimple real Lie group $G$. Then separation of kinematical parts in the functions is fulfilled by means of harmonic analysis related to the group $G$. This separation depends on choice of a coordinate system on the space where a physical system exists. In the paper we review how coordinate systems can be chosen and how the corresponding harmonic analysis can be done. In the first part we consider in detail the case when $G$ is the de Sitter group $S O_{0}(1,4)$. In the second part we show how the corresponding theory can be developed for any noncompact semisimple real Lie group.
\end{abstract}

Key words: representations; eigenfunction expansion; special functions; de Sitter group; semisimple Lie group; coordinate systems; invariant operators

2000 Mathematics Subject Classification: 22E43; 22E46; 33C80; 42C10; 45C05; 81Q10

\section{Introduction}

A symmetry is mathematically described by some group $G$. If a system (a system of particles in quantum mechanics, a system of differential equations, a system of particles in macrophysics etc) admits a symmetry that is given by a group $G$, then a function (functions) that describes this system (wave functions, scattering amplitudes, solutions of a system of differential equations, functions describing a motion in macrophysics etc) contains a part (parts), that is determined by the symmetry (and is independent of a concrete system), and a part (parts) that characterizes a concrete system.

For example, if a system $A$ of differential equations admits a symmetry group $G$, then, using the symmetry, very often one can reduces this system $A$ to simpler system $B$ that does not have a symmetry described by the group $G$. In fact, the system $B$ is obtained from the system $A$ by excluding the symmetry (see, for example, [1]).

As another example, we cite scattering theory (see, for example, [2]). A scattering amplitude decomposes into series in spherical functions $Y_{m}^{l}(\theta, \varphi)$ that characterize a symmetry with respect to the rotation group $S O(3)$. Coefficients of this decomposition are called partial amplitudes. Partial amplitudes depend on smaller number of variables and they characterize the scattering under consideration. There are different collections of partial amplitudes corresponding to different types of scatterings, whereas the functions $Y_{m}^{l}(\theta, \varphi)$ are the same for all types of scatterings.

Separation in the functions, characterizing a system, of a part (parts) depending on symmetry and of a part (parts) characterizing a concrete system is in fact separation of kinematic and dynamical parts. Essential part for studying of a concrete system is a dynamical one. For 
this reason, separation of a dynamical part in a function (in functions), characterizing a given physical system, is a very important procedure. For example, a dynamical part in scattering theory is represented by partial amplitudes which can be directly compared with experimental data.

In a simplest case, separation of kinematic and dynamical parts is a representation of functions, characterizing a system, as a product of kinematic and dynamical parts. It is a degenerate case.

In a more general case (when a symmetry group is compact), functions describing a system are represented as a sum of products of kinematic and dynamical parts (it is a case in the scattering theory).

A main aim of this paper is separation of kinematic parts in functions, describing a system, and studying these parts. The first step in separation of parts, depending on symmetries, is choosing variables in such a way that a part of these variables corresponds to the symmetries (kinematical variables) and remaining ones correspond to dynamics of the system. Further on, one studies harmonic analysis of the kinematical part. Under such analysis, functions describing a physical system are considered as functions depending only on kinematical variables, that is, one pays no attention to dynamical variables.

In the framework of the harmonic analysis of the kinematical part, the functions are expanded into basis functions which are common eigenfunctions of a collection of self-adjoint operators that are determined by the symmetry group. It is clear that coefficients of such expansion depend on dynamical variables.

Such collection of self-adjoint operators is not determined uniquely. This collection depends on kinematical variables. Kinematical variables are not determined uniquely too. As a rule, choice of kinematical variables depends on a dynamical problem that has to be solved.

There exists a one-to-one correspondence between the following collections:

(a) a collection of kinematical variables;

(b) a chain of subgroups of the symmetry group $G$;

(c) a collection of self-adjoint differential operators that are, as a rule, Casimir operators of the group $G$ and of members of the chain of subgroups. These Casimir operators are very often Laplace operators expressed in the corresponding coordinate systems.

A description of such triples for an example of the sphere $S^{n-1}$ in the $n$-dimensional Euclidean space with the symmetry group $S O(n)$ is given in [3,4]. Coordinates (kinematical variables) in this case are polyspherical. There are different types of polyspherical coordinates. Laplace operators on the corresponding manifolds of $S^{n-1}$ expressed in the corresponding polyspherical coordinates serve as a collection of self-adjoint operators. Subgroups of the rotation group $S O(n)$ (corresponding to the chosen type of polyspherical coordinates) with successive inclusions serve as a chain of subgroups of $S O(n)$. Note that harmonic analysis of functions given on quotient spaces of the group $S O(n)$ is used extensively in nuclear physics (see [5, 6] and references therein).

In this paper we review the case when a symmetry group $G$ is a simple noncompact Lie group (for example, the Lorentz group, the de Sitter group, the conformal group etc). The case when $G$ coincides with the motion group $S O_{0}(1,2)$ of the upper sheet of the hyperboloid in the 3-dimensional Minkowski space-time is simple and well-known (see, for example, [7, Chapter 7] and [8]). The case when $G=S O_{0}(1,3)$ is also well-studied (see, for example, $\left.[9,10,11]\right)$. In the relativistic physics, the groups $S O(3), S O_{0}(1,2), I S O(3)$ and $S O_{0}(1,3)$ appear as little groups for the Poincaré group $I S O_{0}(1,3)$ (see $[12,13,14]$ ).

As an example, in the first part of the paper we consider in detail the case when $G$ is the de Sitter group $S O_{0}(1,4)$ that is a motion group of the 5-dimensional Minkowski space-time. This space is a base space for the Caluza-Klein theory. 
In the second part of the paper we consider the case when $G$ is a generic linear simple noncompact real Lie group. Then $G$ is a group of $n \times n$ matrices, where $n$ is some positive integer. We define a hyperboloid and a cone with motion group $G$ and consider how different coordinate systems can be determined on them. Then we perform harmonic analysis of functions given on these general hyperboloids and cones in these coordinate systems. For this purpose, the general harmonic analysis on semisimple noncompact Lie groups [15] and on the corresponding homogeneous spaces [16] is used. Different coordinate systems on a hyperboloid and on a cone with a motion group $G$ are connected to different decompositions of the group $G$ into products of its subgroups (the Iwasawa decomposition, the Cartan decomposition, the generalized Cartan decomposition etc).

The basis for this review is our books [3, 17]. Our consideration is closely related to special functions and orthogonal polynomials, especially with those special functions which admit orthogonality relations. In [3, 17] and also in $[7,18,19]$ one can find a detailed description of the relation between the group representation and special functions. The basic information on special functions and orthogonal polynomials may be found in [20, 21].

\section{The de Sitter group $S O_{0}(1,4)$ and its representations}

The de Sitter group $S O_{0}(1,4)$ consists of all real $5 \times 5$ matrices $g$ with $\operatorname{det} g=1$ which leave invariant the quadratic form

$$
x_{0}^{2}-x_{1}^{2}-x_{2}^{2}-x_{3}^{2}-x_{4}^{2} .
$$

The Lie algebra so $(1,4)$ of $S O_{0}(1,4)$ consists of all real matrices

$$
\left[\begin{array}{ccccc}
0 & a_{01} & a_{02} & a_{03} & a_{04} \\
a_{01} & 0 & -a_{12} & -a_{13} & -a_{14} \\
a_{02} & a_{12} & 0 & -a_{23} & -a_{24} \\
a_{03} & a_{13} & a_{23} & 0 & -a_{34} \\
a_{04} & a_{14} & a_{24} & a_{34} & 0
\end{array}\right]
$$

Thus, the Lie algebra so(1,4) is spanned upon the basis elements

$$
\begin{aligned}
& L_{r s}=-e_{r s}+e_{s r}, \quad s, r=1,2,3,4, \quad s<r, \\
& L_{0 r}=e_{0 r}+e_{r 0}, \quad r=1,2,3,4,
\end{aligned}
$$

where $e_{r s}$ is a matrix with matrix elements $\left(e_{r s}\right)_{p q}=\delta_{r p} \delta_{s q}$. The basis elements (2.2) and (2.3) satisfy the commutation relations

$$
\left[L_{\mu \nu}, L_{\rho \delta}\right]=g_{\nu \rho} L_{\mu \delta}+g_{\mu \delta} L_{\nu \rho}-g_{\mu \rho} L_{\nu \delta}-g_{\nu \delta} L_{\mu \rho}, \quad \rho, \mu, \nu, \delta=0,1,2,3,4,
$$

where $g_{k 0}=g_{0 k}=\delta_{0 k}, g_{k s}=-\delta_{k s}, k, s=1,2,3,4$. The maximal compact subgroup $K$ of $S O_{0}(1,4)$ is isomorphic to the group $S O(4)$ and consists of matrices

$$
\left(\begin{array}{ll}
1 & 0 \\
0 & k
\end{array}\right), \quad k \in S O(4)
$$

In construction of representations of the group $S O_{0}(1,4)$ one uses the Cartan decomposition of the Lie algebra so(1,4) and the Iwasawa decomposition of $S O_{0}(1,4)$. In the Cartan decomposition so $(1,4)=\operatorname{so}(4)+\mathfrak{p}$ the subspace $\mathfrak{p}$ is spanned by basis elements (2.3). Let $\mathfrak{a}$ be a maximal commutative subalgebra in $\mathfrak{p}$. This subalgebra is one-dimensional. The matrix $L_{04}$ can be taken 
as a basis element of $\mathfrak{a}$. The subgroup $A=\exp \mathfrak{a}$ is important in the representation theory of the group $S O_{0}(1,4)$. This subgroup consists of matrices

$$
\left[\begin{array}{ccccc}
\cosh \alpha & 0 & 0 & 0 & \sinh \alpha \\
0 & 1 & 0 & 0 & 0 \\
0 & 0 & 1 & 0 & 0 \\
0 & 0 & 0 & 1 & 0 \\
\sinh \alpha & 0 & 0 & 0 & \cosh \alpha
\end{array}\right], \quad 0 \leqslant \alpha<\infty
$$

Using the commutation relations (2.4), one directly checks that the Lie subalgebra $\mathfrak{n}$ of so(1,4) with the basis $L_{01}+L_{14}, L_{02}+L_{24}, L_{03}+L_{34}$ is nilpotent and, moreover, commutative. The subgroup $N=\exp \mathfrak{n}$ of $S O_{0}(1,4)$ consists of the matrices

$$
\left[\begin{array}{ccccc}
1+\left(r^{2}+s^{2}+t^{2}\right) / 2 & t & r & s & -\left(r^{2}+s^{2}+t^{2}\right) / 2 \\
t & 1 & 0 & 0 & -t \\
r & 0 & 1 & 0 & -r \\
s & 0 & 0 & 1 & -s \\
\left(r^{2}+s^{2}+t^{2}\right) / 2 & t & r & s & 1-\left(r^{2}+s^{2}+t^{2}\right) / 2
\end{array}\right]
$$

with $t, r, s \in \mathbb{R}$. The subgroups $K \equiv S O(4), A$ and $N$ determine the Iwasawa decomposition

$$
S O_{0}(1,4)=S O(4) \cdot N A
$$

of $S O_{0}(1,4)$. The subgroup $M$ of $S O(4) \subset S O_{0}(1,4)$, whose elements commute with elements of $A$, is isomorphic to the group $S O(3)$. The subgroup

$$
P=S O(3) \cdot N A
$$

is called a parabolic subgroup of $S O_{0}(1,4)$. This subgroup is used for construction of irreducible unitary representations of $S O_{0}(1,4)$.

Now we construct representations $\pi_{\delta \lambda}$ of the principal nonunitary series (and, consequently, of the principal unitary series) of the group $S O_{0}(1,4)$. These representations are constructed by means of irreducible unitary representations $\delta$ of the subgroup $S O(3)$ and of complex linear forms $\lambda$ on the subalgebra a. Since irreducible unitary representations $\delta$ of the subgroup $S O(3)$ are given by a non-negative integer or half-integer $l$ and a linear form $\lambda$ is given by a complex number $\sigma=\lambda\left(L_{04}\right)$, then the representations $\pi_{\delta \lambda}$ are determined by the numbers $l$ and $\sigma$. For this reason, we use the notation $\pi_{l \sigma}$ for the representations $\pi_{\delta \lambda}$. We shall use only those representations $\pi_{l \sigma}$ for which $l=0$. These representations will be denoted by $\pi^{\sigma}$. The representations $\pi^{\sigma}$ are characterized by the property that the restriction $\pi^{\sigma} \downarrow_{S O(4)}$ contains the trivial (one-dimensional) representation of the subgroup $S O(4)$. Moreover, they exhaust all principal nonunitary series representations of $S O_{0}(1,4)$ with this property. These representations are called representations of class 1 with respect to $S O(4)$.

For $\sigma=\mathrm{i} \rho-\frac{3}{2}, \rho \in \mathbb{R}$, the representations $\pi^{\sigma}$ are unitary and belong to the principal unitary series.

The group $S O_{0}(1,4)$ has two independent Casimir operators

$$
\begin{aligned}
F= & L_{12}^{2}+L_{13}^{2}+L_{14}^{2}+L_{23}^{2}+L_{24}^{2}+L_{34}^{2}-L_{01}^{2}-L_{02}^{2}-L_{03}^{2}-L_{04}^{2}, \\
W= & \left(L_{12} L_{24}-L_{13} L_{24}+L_{14} L_{23}\right)^{2} \\
& -\left(L_{02} L_{34}-L_{03} L_{24}+L_{04} L_{23}\right)^{2}-\left(L_{01} L_{34}-L_{03} L_{14}+L_{04} L_{13}\right)^{2} \\
& -\left(L_{01} L_{24}-L_{02} L_{14}+L_{04} L_{12}\right)^{2}-\left(L_{01} L_{23}-L_{02} L_{13}+L_{03} L_{12}\right)^{2} .
\end{aligned}
$$


It is known (see, for example, [22]) that the Casimir operator $W$ vanishes on the representations $\pi^{\sigma}$ of $S O_{0}(1,4)$. The Casimir operator $F$ takes on the representations $\pi^{\sigma}$ the values $-\sigma(\sigma+3)$.

Under restriction to the subgroup $K=S O(4)$, the representation $\pi^{\sigma}$ decomposes into a direct sum of irreducible representations of $S O(4)$ with highest weights $(m, 0), m=0,1,2, \ldots$ The group $S O(4)$ is locally isomorphic to the group $S O(3) \times S O(3)$. The irreducible representation of $S O(4)$ with the highest weight $(m, 0)$, as a representation of $S O(3) \times S O(3)$, is given by the numbers $\frac{m}{2}$ and $\frac{m}{2}$.

Let us consider representations of $S O_{0}(1,4)$ realized on functions on the upper sheet of the two-sheeted hyperboloid and on functions on the upper sheet of the cone. The upper sheet $H_{+}^{4}$ of the two-sheeted hyperboloid $H^{4}$ can be obtained as a quotient space $S O_{0}(1,4) / S O(4)$. In order to show this, we consider the upper sheet $H_{+}^{4}$,

$$
H_{+}^{4}: x_{0}^{2}-x_{1}^{2}-x_{2}^{2}-x_{3}^{2}-x_{4}^{2}=1, \quad x_{0}>0,
$$

and the point $x^{0}=(1,0,0,0,0)$ on it. Elements of $S O_{0}(1,4)$ transform the hyperboloid $H_{+}^{4}$ into $H_{+}^{4}$. Besides, for any two points $x^{\prime}$ and $x^{\prime \prime}$ of $H_{+}^{4}$ there exists an element $g \in S O_{0}(1,4)$ such that $g x^{\prime}=x^{\prime \prime}$, that is, the group $S O_{0}(1,4)$ acts transitively on $H_{+}^{4}$. A set of points of $S O_{0}(1,4)$, leaving the point $x^{0}$ invariant, coincides with the subgroup $S O(4)$. Therefore, $H_{+}^{4}$ can be identified with $S_{0}(1,4) / S O(4)$. Note that on $H_{+}^{4}$ the 4 -dimensional Lobachevsky space $\mathcal{L}^{4}$ is realized, which is also called the de Sitter space. As to the spherical functions on this space, see in [23].

One constructs on functions $f(x)$ given on $H_{+}^{4}$ the quasi-regular representation of the group $S O_{0}(1,4)$. Let $L^{2}\left(H_{+}^{4}\right)$ be the Hilbert space of functions on $H_{+}^{4}$ with the scalar product

$$
\left\langle f_{1}, f_{2}\right\rangle=\int_{H_{+}^{4}} f_{1}(x) \overline{f_{2}(x)} d \mu(x),
$$

where $d \mu(x)$ is an invariant (with respect to $S O_{0}(1,4)$ ) measure on $H_{+}^{4}$. This measure is determined by the formula

$$
d \mu(x)=d^{4} x / x_{0}=d x_{1} d x_{2} d x_{3} d x_{4} / x_{0} .
$$

The quasi-regular representation $\pi$ of $S O_{0}(1,4)$ is given on $L^{2}\left(H_{+}^{4}\right)$ by the formula

$$
\pi(g) f(x)=f\left(g^{-1} x\right), \quad x \in H_{+}^{4} .
$$

It is easy to show that this representation is unitary. However, this representation is reducible and decomposes [24] into a direct integral of irreducible unitary representations $\pi^{\sigma}\left(\sigma=-\frac{3}{2}+i \rho\right.$, $0 \leq \rho<\infty)$.

The quasi-regular representation of $S O_{0}(1,4)$ on the Hilbert space $L^{2}\left(C_{+}^{4}\right)$ of functions on the upper sheet $C_{+}^{4}$ of the cone $C^{4}$,

$$
C_{+}^{4}: x_{0}^{2}-x_{1}^{2}-x_{2}^{2}-x_{3}^{2}-x_{4}^{2}=0, \quad x_{0}>0,
$$

is constructed analogously:

$$
\pi(g) f(x)=f\left(g^{-1} x\right), \quad x \in C_{+}^{4} .
$$

The cone $C_{+}^{4}$ can be identified with the homogeneous space $S O_{0}(1,4) /(S O(3) \times N)$. In order to check this we have to take the point $x^{0}=(1,0,0,0,1) \in C_{+}^{4}$ and to verify that $S O(3) \times N$ is a subgroup of $S O_{0}(1,4)$ whose elements leave $x^{0}$ invariant. The subgroup $S O(3) \times N$ is isomorphic to the group $I S O(3)$ of motions of the 3-dimensional Euclidean space. 
The representation $(2.12)$ is unitary with respect to the scalar product

$$
\left\langle f_{1}, f_{2}\right\rangle=\int_{C_{+}^{4}} f_{1}(x) \overline{f_{2}(x)} d \mu(x)
$$

on $L^{2}\left(C_{+}^{4}\right)$. Here $d \mu(x)=d^{4} x / x_{0}$ is an invariant (with respect to $S O_{0}(1,4)$ ) measure on $C_{+}^{4}$. This representation is also reducible. Irreducible unitary representations of $S O_{0}(1,4)$ can be constructed on spaces of homogeneous functions on the cone (see, for example, [25]). Representations of the group $S O_{0}(1,4)$ (as well as of the group $S O_{0}(1, p)$ ) are well investigated (see $[26,27,28,29,30])$.

Under exposition of harmonic analysis of functions on the homogeneous space $H_{+}^{4}$ of the group $S O_{0}(1,4)$ we shall use the method developed by Vilenkin and Smorodinsky [9] for the Lorentz group $S O_{0}(1,3)$ and the results of the paper [24] related to the integral geometry.

The de Sitter group $S O_{0}(1,4)$ is used in different branches of contemporary physics [31]. This is a group of motions of a symmetric Riemannian space-time, which generalizes the Poincaré group $I S O_{0}(1,3)$ [32]. The de Sitter group $S O_{0}(1,4)$ is known as a group of invariance of non-relativistic hydrogen atom since it contains the dynamical group $S O_{0}(1,3)$ (continuous spectrum) and the dynamical group $S O(4)$ (discrete spectrum) [33, 34]. There exist also other directions of applications of the de Sitter group [35].

\section{Subgroups of the group $S O_{0}(1,4)$}

Below we shall construct different bases of the space $L^{2}\left(H_{+}^{4}\right)$ and shall derive formulas for expansion of functions of $L^{2}\left(H_{+}^{4}\right)$ in these bases. However, we shall consider not arbitrary bases of $L^{2}\left(H_{+}^{4}\right)$ but only those of them which correspond to chains of subgroups of the group $S O_{0}(1,4)$. These bases can be constructed also by means of collections of commuting self-adjoint operators. Basis functions on $H_{+}^{4}$ consist of common eigenfunctions of these collections of operators. Bases of the space $L^{2}\left(C_{+}^{4}\right)$ are constructed analogously.

A standard way for construction of a collection of self-adjoint operators is to construct the corresponding chain of subgroups $G^{\prime} \supset G^{\prime \prime} \supset G^{\prime \prime \prime} \supset \cdots$ of $S O_{0}(1,4)$. Then one creates commuting self-adjoint operators, consisting of Casimir operators of the subgroups of the chain. To each such chain there corresponds a collection of self-adjoint operators.

We consider these collections of operators as differential operators on homogeneous spaces of the group $S O_{0}(1,4)$ (on the hyperboloid $H_{+}^{4}$ or on the cone $C_{+}^{4}$ ). For obtaining these differential operators, we construct coordinate systems such that the corresponding variables can be separated in the differential equations for eigenvalues and eigenfunctions of the collection of operators. To each chain of subgroups (and, therefore, to each collection of self-adjoint operators) there corresponds such coordinate system.

In this section we determine subgroups of the group $S O_{0}(1,4)$ that will be used for construction of chains of subgroups.

In order to deal with self-adjoint operators, we shall use the elements $J_{\mu \nu}=\mathrm{i} L_{\mu \nu}$ of $\operatorname{so}(1,4)$ instead of elements $L_{\mu \nu}$. For 10 generators $J_{\mu \nu}$ of the group $S O_{0}(1,4)$ we introduce the notations

$$
\begin{aligned}
& \mathbf{M}=\left(M_{1} \equiv J_{23}, M_{2} \equiv J_{31}, M_{3} \equiv J_{12}\right) \\
& \mathbf{P}=\left(P_{1} \equiv J_{14}, P_{2} \equiv J_{24}, P_{3} \equiv J_{34}\right) \\
& \mathbf{N}=\left(N_{1} \equiv J_{01}, N_{2} \equiv J_{02}, N_{3} \equiv J_{03}\right) \\
& P_{0}=J_{04}
\end{aligned}
$$

In these notations we have the following expressions for Casimir operators:

$$
F=\left(P_{0}^{2}+\mathbf{N}^{2}\right)-\left(\mathbf{P}^{2}+\mathbf{M}^{2}\right),
$$




$$
W=(\mathbf{M} \cdot \mathbf{P})^{2}-\left(P_{0} \mathbf{M}-\mathbf{P} \times \mathbf{N}\right)^{2}-(\mathbf{M} \cdot \mathbf{N})^{2} .
$$

Commutation relations for the generators $\mathbf{M}, \mathbf{P}, \mathbf{N}$ and $P_{0}$ are of the form

$$
\begin{aligned}
& {\left[M_{k}, M_{l}\right]=\mathrm{i} \varepsilon_{k l m} M_{m}, \quad\left[N_{k}, N_{l}\right]=-\mathrm{i} \varepsilon_{k l m} M_{m}, \quad\left[P_{k}, P_{l}\right]=\mathrm{i} \varepsilon_{k l m} M_{m},} \\
& {\left[M_{k}, N_{l}\right]=\mathrm{i} \varepsilon_{k l m} N_{m}, \quad\left[M_{k}, P_{l}\right]=\mathrm{i} \varepsilon_{k l m} P_{M},} \\
& {\left[M_{k}, N_{k}\right]=\left[M_{k}, P_{k}\right]=\left[M_{k}, P_{0}\right]=0,} \\
& {\left[P_{0}, N_{k}\right]=\mathrm{i} P_{k}, \quad\left[P_{0}, P_{k}\right]=\mathrm{i} N_{k}, \quad\left[P_{k}, N_{l}\right]=\mathrm{i} \delta_{k l} P_{0}}
\end{aligned}
$$

where $\varepsilon_{k l m}, k, l, m=1,2,3$, is the antisymmetric tensor of the third order equal to 0 or \pm 1 . Using the generators $\mathbf{M}, \mathbf{P}, \mathbf{N}$ and $P_{0}$ we construct subgroups of the group $S O_{0}(1,4)$.

Subgroup $S O(3)$. This subgroup corresponds to the generators $\mathbf{M}=\left(M_{1}, M_{2}, M_{3}\right)$ of the Lie algebra so(1,4):

$$
\left[M_{k}, M_{l}\right]=\mathrm{i} \varepsilon_{k l m} M_{m} .
$$

Subgroup $S O(4)$. There are the generators $\mathbf{M}=\left(M_{1}, M_{2}, M_{3}\right)$ and $\mathbf{P}=\left(P_{1}, P_{2}, P_{3}\right)$ of the Lie algebra so $(1,4)$ corresponding to this subgroup:

$$
\left[M_{k}, M_{l}\right]=\mathrm{i} \varepsilon_{k l m} M_{m}, \quad\left[M_{k}, P_{l}\right]=\mathrm{i} \varepsilon_{k l m} P_{m}, \quad\left[P_{k}, P_{l}\right]=\mathrm{i} \varepsilon_{k l m} M_{m} .
$$

Taking the linear combinations $\mathbf{V}=(\mathbf{M}+\mathbf{P}) / 2, \mathbf{V}^{\prime}=(\mathbf{M}-\mathbf{P}) / 2$, we obtain instead of (3.7) the relations

$$
\left[V_{k}, V_{l}\right]=\mathrm{i} \varepsilon_{k l m} V_{m}, \quad\left[V_{k}^{\prime}, V_{l}^{\prime}\right]=\mathrm{i} \varepsilon_{k l m} V_{m}^{\prime}, \quad\left[V_{k}, V_{l}^{\prime}\right]=0,
$$

that is, triples of the operators $\mathbf{V}$ and $\mathbf{V}^{\prime}$ constitute bases of two independent Lie algebras so(3). This means that the group $S O(4)$ is locally isomorphic to $S O(3) \otimes S O(3)$.

Subgroup $S O_{0}(1,3)$. It is the Lorentz group which is generated by $\mathbf{M}=\left(M_{1}, M_{2}, M_{3}\right)$ and $\mathbf{N}=\left(N_{1}, N_{2}, N_{3}\right)$. We have the commutation relations

$$
\left[M_{k}, M_{l}\right]=\mathrm{i} \varepsilon_{k l m} M_{m}, \quad\left[M_{k}, N_{l}\right]=\mathrm{i} \varepsilon_{k l m} N_{m}, \quad\left[N_{k}, N_{l}\right]=-\mathrm{i} \varepsilon_{k l m} M_{m} .
$$

If we introduce the generators $\mathbf{L}^{(1)}=(\mathbf{M}+\mathrm{i} \mathbf{N}) / 2$ and $\mathbf{L}^{(2)}=(\mathbf{M}-\mathrm{i} \mathbf{N}) / 2$, then we obtain the commutation relations for the generators of the Lie algebra so(3):

$$
\left[L_{k}^{(\tau)}, L_{l}^{\left(\tau^{\prime}\right)}\right]=\mathrm{i} \varepsilon_{k l m} L_{m}^{(\tau)} \delta_{\tau \tau^{\prime}}, \quad \tau, \tau^{\prime}=1,2 .
$$

Subgroup $S O_{0}(1,1) \otimes S O(3)$. This subgroup is generated by the generators $\mathbf{M}=\left(M_{1}, M_{2}\right.$, $\left.M_{3}\right), P_{0}$. We have

$$
\left[M_{k}, M_{l}\right]=\mathrm{i} \varepsilon_{k l m} M_{m}, \quad\left[M_{k}, P_{0}\right]=0 .
$$

Subgroup $S O_{0}(1,2) \otimes S O^{\prime}(2)$. This subgroup is generated by the generators $N_{1}, N_{2}, M_{3}, P_{3}$. They satisfy the commutation relations

$$
\begin{aligned}
& {\left[N_{1}, N_{2}\right]=-\mathrm{i} M_{3}, \quad\left[M_{3}, N_{1}\right]=\mathrm{i} N_{2}, \quad\left[M_{3}, N_{2}\right]=-\mathrm{i} N_{1},} \\
& {\left[M_{3}, P_{3}\right]=\left[N_{1}, P_{3}\right]=\left[N_{2}, P_{3}\right]=0 .}
\end{aligned}
$$

The subgroup $S O^{\prime}(2)$ of the group $S O_{0}(1,2) \otimes S O^{\prime}(2)$ is generated by the generator $P_{3}$.

Subgroup $I S O(3)$. We create from the generators $P_{1}, P_{2}, P_{3}$ and $N_{1}, N_{2}, N_{3}$ of the Lie algebra so $(1,4)$ the following linear combinations:

$$
E_{1}=P_{1}+N_{1}, \quad E_{2}=P_{2}+N_{2}, \quad E_{3}=P_{3}+N_{3} .
$$


It is easy to show that two triples of generators $\mathbf{E}=\left(E_{1}, E_{2}, E_{3}\right)$ and $\mathbf{M}=\left(M_{1}, M_{2}, M_{3}\right)$ satisfy the commutation relations for the basis generators of the Lie algebra $I S O(3)$, which is the Lie algebra of the group of motions of the 3 -dimensional Euclidean space $\mathbb{R}^{3}$. These relations are

$$
\left[M_{k}, M_{l}\right]=\mathrm{i} \varepsilon_{k l m} M_{m}, \quad\left[M_{k}, E_{l}\right]=\mathrm{i} \varepsilon_{k l m} E_{m}, \quad\left[E_{k}, E_{l}\right]=0 .
$$

This group is a semidirect product of the group $S O(3)$ and the group $T(3)$ of shifts in $\mathbb{R}^{3}$, $I S O(3)=S O(3) \times T(3)$. Note that $T(3)$ coincides with the subgroup $N$.

Subgroup $I S O(2)$. Three operators

$$
\mathcal{E}_{1}=E_{1}, \quad \mathcal{E}_{2}=E_{2}, \quad \mathcal{E}_{3}=E_{3}+M_{3}
$$

generate the Lie algebra of the group $I S O(2)$ such that $I S O(2) \subset I S O(3)$. We have

$$
\left[\mathcal{E}_{1}, \mathcal{E}_{2}\right]=0, \quad\left[\mathcal{E}_{1}, \mathcal{E}_{3}\right]=-\mathrm{i} \mathcal{E}_{2}, \quad\left[\mathcal{E}_{2}, \mathcal{E}_{3}\right]=\mathrm{i} \mathcal{E}_{1}
$$

Subgroup $I S O(2) \otimes T_{\perp}$. The set of generators $E_{1}, E_{2}, M_{3}, E_{3}$ satisfying the commutation relations

$$
\left[E_{k}, E_{j}\right]=0, \quad\left[M_{3}, E_{3}\right]=0, \quad\left[M_{3}, E_{2}\right]=-\mathrm{i} E_{1}, \quad\left[M_{3}, E_{1}\right]=\mathrm{i} E_{2}
$$

generate a Lie algebra of the group $I S O(2) \otimes T_{\perp}$ belonging to the group $I S O(3)$. The group $T_{\perp}$ is a group of shifts along of the direct line, which is perpendicular to the plane with the group of motion $I S O(2)$. The generators $M_{3}$ and $E_{3}$ generate the subgroup $S O(2) \otimes T_{\perp}$ of the group $I S O(2) \otimes T_{\perp}$ and we have $\left[M_{3}, E_{3}\right]=0$.

Subgroup $T(3)$. A basis of the Lie algebra of the group $T(3) \subset I S O(3)$ consists of the generators $E_{1}, E_{2}, E_{3}$. Each of the generators $E_{k}$ generates a one-parameter subgroup $T_{k} \subset T(3)$ of shifts along the corresponding coordinate axes.

\section{Coordinate systems on hyperboloid $\boldsymbol{H}_{+}^{4}$ and generators of the group $S_{0}(1,4)$}

In this section we consider coordinate systems on the hyperboloid $H_{+}^{4}$ and find differential form of generators of the de Sitter group $S O_{0}(1,4)$ in an explicit form. Points of the hyperboloid $H_{+}^{4}$ are characterized by 5 orthogonal coordinates $x_{\mu}, \mu=0,1,2,3,4$, such that

$$
H_{+}^{4}:[x, x]:=x_{0}^{2}-x_{1}^{2}-x_{2}^{2}-x_{3}^{2}-x_{4}^{2}=1, \quad x_{0}>0 .
$$

These coordinates are called homogeneous. Since $[x, x]=1$, then the numbers $x_{\mu}$, giving a point $x$ of the space $H_{+}^{4} \equiv \mathcal{L}^{4}$, are in fact its projective coordinates.

New coordinates on $H_{+}^{4}$ will be given by means of relations connecting them with the coordinates $x_{\mu}, \mu=0,1,2,3,4$.

Spherical coordinate system $S$ (coordinates $a, \beta, \theta, \varphi)$ :

$$
\begin{aligned}
& x_{0}=\cosh a, \quad x_{1}=\sinh a \sin \beta \sin \theta \cos \varphi, \quad x_{2}=\sinh a \sin \beta \sin \theta \sin \varphi, \\
& x_{3}=\sinh a \sin \beta \cos \theta, \quad x_{4}=\sinh a \cos \beta, \\
& 0 \leqslant a<\infty, \quad 0 \leqslant \beta, \theta<\pi, \quad 0 \leqslant \phi<2 \pi .
\end{aligned}
$$

Hyperbolic coordinate system $H$ (coordinates $a, b, \theta, \varphi)$ :

$$
x_{0}=\cosh a \cosh b, \quad x_{1}=\cosh a \sinh b \sin \theta \cos \varphi,
$$




$$
\begin{aligned}
& x_{2}=\cosh a \sinh b \sin \theta \sin \varphi, \quad x_{3}=\cosh a \sinh b \cos \theta, \quad x_{4}=\sinh a, \\
& -\infty<a<\infty, \quad 0 \leqslant b<\infty, \quad 0 \leqslant \theta<\pi, \quad 0 \leqslant \varphi<2 \pi .
\end{aligned}
$$

Orispherical coordinate system $O$ (coordinates $a, r, \theta, \varphi)$ :

$$
\begin{aligned}
& x_{0}-x_{4}=e^{a}, \quad x_{0}+x_{4}=e^{-a}+r^{2} e^{a}, \\
& x_{1}=e^{a} r \sin \theta \cos \varphi, \quad x_{2}=e^{a} r \sin \theta \sin \varphi, \quad x_{3}=e^{a} r \cos \theta, \\
& -\infty<a<\infty, \quad 0 \leqslant r<\infty, \quad 0 \leqslant \theta<\pi, \quad 0 \leqslant \varphi<2 \pi .
\end{aligned}
$$

Orispherically-cylindric coordinate system $O C$ (coordinates $a, \xi, z, \varphi)$ :

$$
\begin{array}{ll}
x_{0}-x_{4}=e^{a}, & x_{0}+x_{4}=e^{-a}+\left(\xi^{2}+z^{2}\right) e^{a}, \\
x_{1}=e^{a} \xi \cos \varphi, & x_{2}=e^{a} \xi \sin \varphi, \quad x_{3}=e^{a} \xi, \\
-\infty<a<\infty, & 0 \leqslant \xi<\infty, \quad-\infty<z<\infty,
\end{array}
$$

Orispherically-translational coordinate system $O T$ (coordinates $a, y_{1}, y_{2}, y_{3}$ ):

$$
\begin{aligned}
& x_{0}-x_{4}=e^{a}, \quad x_{0}+x_{4}=e^{-a}+y^{2} e^{a}, \\
& x_{1}=e^{a} y_{1}, \quad x_{2}=e^{a} y_{2}, \quad p_{3}=e^{a} y_{3}, \\
& y^{2}=y_{1}^{2}+y_{2}^{2}+y_{3}^{2}, \quad-\infty<a<\infty, \quad-\infty<y_{i}<\infty .
\end{aligned}
$$

Cylindric coordinate system $C$ (coordinates $a, b, \theta, \varphi)$ :

$$
\begin{array}{ll}
x_{0}=\cosh a \cosh b, \quad x_{1}=\sinh a \sin \theta \cos \varphi, & x_{2}=\sinh a \sin \theta \sin \varphi, \\
x_{3}=\sinh a \cos \theta, \quad x_{4}=\cosh a \sinh b, & \\
0 \leqslant a<\infty, \quad-\infty<b<\infty, \quad 0 \leqslant \theta<\pi, & 0 \leqslant \varphi<2 \pi .
\end{array}
$$

Spherically-hyperbolic coordinate system $S H$ (coordinates $a, b, \varphi, \Phi)$ :

$$
\begin{aligned}
& x_{0}=\cosh a \cosh b, \quad x_{1}=\cosh a \sinh b \cos \varphi, \quad x_{2}=\cosh a \sinh b \sin \varphi, \\
& x_{3}=\sinh a \cos \Phi, \quad x_{4}=\sinh a \sin \Phi, \\
& 0 \leqslant a, b<\infty, \quad 0 \leqslant \varphi, \Phi<2 \pi .
\end{aligned}
$$

Now we go to the representation $(2.11)$ of the group $S O_{0}(1,4)$ realized on the space $L^{2}\left(H_{+}^{4}\right)$. This representation gives in fact a realization of this group on the hyperboloid $H_{+}^{4}$. Functions of the space $L^{2}\left(H_{+}^{4}\right)$ can be considered as functions of parameters of any of the coordinate systems on $H_{+}^{4}$. We shall give a differential form of infinitesimal operators of the representation (2.11) or, equivalently, a differential form of generators of the group $S O_{0}(1,4)$, realized on $H_{+}^{4}$.

Let $I$ be an infinitesimal generator of the group $S O_{0}(1,4)$, realized on $H_{+}^{4}$. Then $\exp t I$ is a one-parameter subgroup of $S O_{0}(1,4)$. The operator $\pi(\exp t I)$ acts on $L^{2}\left(H_{+}^{4}\right)$ as

$$
\pi(\exp t I) f(x)=f\left((\exp t I)^{-1} x\right) .
$$

Since $\pi(I)=\frac{d}{d t} \pi(\exp t I)_{t=0}$, then

$$
\pi(I) f(x)=\lim _{t \rightarrow 0} \frac{f\left((\exp t I)^{-1} x\right)-f(x)}{t} .
$$

Thus, in the homogeneous coordinates $x_{\mu}$ we have

$$
J_{r s}=-\mathrm{i}\left(x_{r} \frac{\partial}{\partial x_{s}}-x_{s} \frac{\partial}{\partial x_{r}}\right), \quad J_{0 s}=-\mathrm{i}\left(x_{0} \frac{\partial}{\partial x_{s}}+x_{s} \frac{\partial}{\partial x_{0}}\right) .
$$


(Here and below we write down the operators $\pi(I)$ as $I$.) Substituting into (4.9) the expressions for $x_{\mu}, \mu=0,1,2,3,4$, in terms of the corresponding coordinates we find a differential form of the generators $I$ in these coordinates. Let us give the result of such calculation.

S-system:

$$
\begin{aligned}
M_{1}= & -\mathrm{i}\left(-\sin \varphi \frac{\partial}{\partial \theta}-\cot \theta \cos \varphi \frac{\partial}{\partial \varphi}\right), \quad M_{2}=-\mathrm{i}\left(\cos \varphi \frac{\partial}{\partial \theta}-\cot \theta \sin \varphi \frac{\partial}{\partial \varphi}\right), \\
M_{3}= & -\mathrm{i} \frac{\partial}{\partial \varphi}, \quad P_{0}=-\mathrm{i}\left(\cos \beta \frac{\partial}{\partial a}-\operatorname{coth} a \sin \beta \frac{\partial}{\partial \beta}\right), \\
P_{1}= & -\mathrm{i}\left(-\sin \theta \cos \varphi \frac{\partial}{\partial \beta}-\cot \beta \cos \theta \cos \varphi \frac{\partial}{\partial \theta}+\cot \beta \frac{\sin \varphi}{\sin \theta} \frac{\partial}{\partial \varphi}\right), \\
P_{2}= & -\mathrm{i}\left(-\sin \theta \sin \varphi \frac{\partial}{\partial \beta}-\cot \beta \cos \theta \sin \varphi \frac{\partial}{\partial \theta}-\cot \beta \frac{\cos \varphi}{\sin \theta} \frac{\partial}{\partial \varphi}\right), \\
P_{3}= & -\mathrm{i}\left(-\cos \theta \frac{\partial}{\partial \beta}+\cot \beta \sin \theta \frac{\partial}{\partial \theta}\right), \\
N_{1}= & -\mathrm{i}\left(\sin \beta \sin \theta \cos \varphi \frac{\partial}{\partial a}+\operatorname{coth} a \cos \beta \sin \theta \cos \varphi \frac{\partial}{\partial \beta}\right. \\
& \left.+\operatorname{coth} a \frac{\cos \theta \cos \varphi}{\sin \beta} \frac{\partial}{\partial \theta}-\operatorname{coth} a \frac{\sin \varphi}{\sin \beta \sin \theta} \frac{\partial}{\partial \varphi}\right), \\
N_{2}= & -\mathrm{i}\left(\sin \beta \sin \theta \sin \varphi \frac{\partial}{\partial a}+\operatorname{coth} a \cos \beta \sin \theta \sin \varphi \frac{\partial}{\partial \beta}\right. \\
& \left.+\operatorname{coth} a \frac{\cos \theta \sin \varphi}{\sin \beta} \frac{\partial}{\partial \theta}+\operatorname{coth} a \frac{\cos \varphi}{\sin \beta \sin \theta} \frac{\partial}{\partial \varphi}\right), \\
N_{3}= & -\mathrm{i}\left(\sin \beta \cos \theta \frac{\partial}{\partial a}+\operatorname{coth} a \cos \beta \cos \theta \frac{\partial}{\partial \beta}-\operatorname{coth} a \frac{\sin \theta}{\sin \beta} \frac{\partial}{\partial \theta}\right) .
\end{aligned}
$$

\section{$H$-system:}

$$
\begin{aligned}
M_{1}= & -\mathrm{i}\left(-\sin \varphi \frac{\partial}{\partial \theta}-\cot \theta \cos \varphi \frac{\partial}{\partial \varphi}\right), \quad M_{2}=-\mathrm{i}\left(\cos \varphi \frac{\partial}{\partial \theta}-\cot \theta \sin \varphi \frac{\partial}{\partial \varphi}\right), \\
M_{3}= & -\mathrm{i} \frac{\partial}{\partial \varphi}, \quad P_{0}=-\mathrm{i}\left(\cosh b \frac{\partial}{\partial a}-\tanh a \sinh b \frac{\partial}{\partial b}\right), \\
P_{1}= & -\mathrm{i}\left(\sinh b \sin \theta \cos \varphi \frac{\partial}{\partial a}-\tanh a \cosh b \sin \theta \cos \varphi \frac{\partial}{\partial b}\right. \\
& \left.-\tanh a \frac{\cos \theta \cos \varphi}{\sinh b} \frac{\partial}{\partial \theta}+\tanh a \frac{\sin \varphi}{\sinh b \sin \theta} \frac{\partial}{\partial \varphi}\right), \\
P_{2}= & -\mathrm{i}\left(\sinh b \sin \theta \sin \varphi \frac{\partial}{\partial a}-\tanh a \cosh b \sin \theta \sin \varphi \frac{\partial}{\partial b}\right. \\
& \left.-\tanh a \frac{\cos \theta \sin \varphi}{\sinh b} \frac{\partial}{\partial \theta}-\tanh a \frac{\cos \varphi}{\sinh b \sin \theta} \frac{\partial}{\partial \varphi}\right), \\
P_{3}= & -\mathrm{i}\left(\sinh b \cos \theta \frac{\partial}{\partial a}-\tanh a \cosh b \cos \theta \frac{\partial}{\partial b}+\tanh a \frac{\sin \theta}{\sinh b} \frac{\partial}{\partial \theta}\right), \\
N_{1}= & -\mathrm{i}\left(\sin \theta \cos \varphi \frac{\partial}{\partial b}+\operatorname{coth} b \cos \theta \cos \varphi \frac{\partial}{\partial \theta}-\operatorname{coth} b \frac{\sin \varphi}{\sin \theta} \frac{\partial}{\partial \varphi}\right), \\
N_{2}= & -\mathrm{i}\left(\sin \theta \sin \varphi \frac{\partial}{\partial b}+\operatorname{coth} b \cos \theta \sin \varphi \frac{\partial}{\partial \theta}+\operatorname{coth} b \frac{\cos \varphi}{\sin \theta} \frac{\partial}{\partial \varphi}\right), \\
N_{3}= & -\mathrm{i}\left(\cos \theta \frac{\partial}{\partial b}-\operatorname{coth} b \sin \theta \frac{\partial}{\partial \theta}\right) .
\end{aligned}
$$


O-system:

$$
\begin{aligned}
M_{1}= & -\mathrm{i}\left(-\sin \varphi \frac{\partial}{\partial \theta}-\cot \theta \cos \varphi \frac{\partial}{\partial \varphi}\right), \quad M_{2}=-\mathrm{i}\left(\cos \varphi \frac{\partial}{\partial \theta}-\cot \theta \sin \varphi \frac{\partial}{\partial \varphi}\right) \\
M_{3}= & -\mathrm{i} \frac{\partial}{\partial \varphi}, \quad P_{0}=-\mathrm{i}\left(-\frac{\partial}{\partial a}+r \frac{\partial}{\partial r}\right) \\
P_{1}= & -\mathrm{i}\left\{-r \sin \theta \cos \varphi \frac{\partial}{\partial a}+\frac{e^{-a}}{2}\left[-e^{-a}+\left(r^{2}+1\right) e^{a}\right] \sin \theta \cos \varphi \frac{\partial}{\partial r}\right. \\
& \left.-\frac{e^{-a}}{2 r}\left[e^{-a}+\left(r^{2}-1\right) e^{a}\right] \cos \theta \cos \varphi \frac{\partial}{\partial \theta}+\frac{e^{-a}}{2 r}\left[e^{-a}+\left(r^{2}-1\right) e^{a}\right] \frac{\sin \varphi}{\sin \theta} \frac{\partial}{\partial \varphi}\right\} \\
P_{2}= & -\mathrm{i}\left\{-r \sin \theta \sin \varphi \frac{\partial}{\partial a}+\frac{e^{-a}}{2}\left[-e^{-a}+\left(r^{2}+1\right) e^{a}\right] \sin \theta \sin \varphi \frac{\partial}{\partial r}\right. \\
& \left.-\frac{e^{-a}}{2 r}\left[e^{-a}+\left(r^{2}-1\right) e^{a}\right] \cos \theta \sin \varphi \frac{\partial}{\partial \theta}-\frac{e^{-a}}{2 r}\left[e^{-a}+\left(r^{2}-1\right) e^{a}\right] \frac{\cos \varphi}{\sin \theta} \frac{\partial}{\partial \varphi}\right\} \\
P_{3}= & -\mathrm{i}\left\{-r \cos \theta \frac{\partial}{\partial a}+\frac{e^{-a}}{2}\left[-e^{-a}+\left(r^{2}+1\right) e^{a}\right] \cos \theta \frac{\partial}{\partial r}\right. \\
& \left.+\frac{e^{-a}}{2 r}\left[e^{-a}+\left(r^{2}-1\right) e^{a}\right] \sin \theta \frac{\partial}{\partial \theta}\right\}, \\
N_{1}= & -\mathrm{i}\left\{r \sin \theta \cos \varphi \frac{\partial}{\partial a}-\frac{e^{-a}}{2}\left[-e^{-a}+\left(r^{2}-1\right) e^{a}\right] \sin \theta \cos \varphi \frac{\partial}{\partial r}\right. \\
& \left.+\frac{e^{-a}}{2 r}\left[e^{-a}+\left(r^{2}+1\right) e^{a}\right] \cos \theta \cos \varphi \frac{\partial}{\partial \theta}-\frac{e^{-a}}{2 r}\left[e^{-a}+\left(r^{2}+1\right) e^{a}\right] \frac{\sin \varphi}{\sin \theta} \frac{\partial}{\partial \varphi}\right\} \\
& \left.+\frac{e^{-a}}{2 r}\left[e^{-a}+\left(r^{2}+1\right) e^{a}\right] \cos \theta \sin \varphi \frac{\partial}{\partial \theta}+\frac{e^{-a}}{2 r}\left[e^{-a}+\left(r^{2}+1\right) e^{a}\right] \frac{\cos \varphi}{\sin \theta} \frac{\partial}{\partial \varphi}\right\} \\
N_{3}= & -\mathrm{i}\left\{r \cos \theta \frac{\partial}{\partial a}-\frac{e^{-a}}{2}\left[-e^{-a}+\left(r^{2}-1\right) e^{a}\right] \cos \theta \frac{\partial}{\partial r}\right. \\
N_{2}= & -\mathrm{i}\left\{r \sin \theta \sin \varphi \frac{\partial}{\partial a}-\frac{e^{-a}}{2}\left[-e^{-a}+\left(r^{2}-1\right) e^{a}\right] \sin \theta \sin \varphi \frac{\partial}{\partial r}\right. \\
& \\
&
\end{aligned}
$$

\section{$O C$-system:}

$$
\begin{aligned}
M_{1}= & -\mathrm{i}\left(-z \sin \varphi \frac{\partial}{\partial \xi}+\xi \sin \varphi \frac{\partial}{\partial z}-z \frac{\cos \varphi}{\xi} \frac{\partial}{\partial \varphi}\right), \\
M_{2}= & -\mathrm{i}\left(z \cos \varphi \frac{\partial}{\partial \xi}-\xi \cos \varphi \frac{\partial}{\partial z}-z \frac{\sin \varphi}{\xi} \frac{\partial}{\partial \varphi}\right), \quad M_{3}=-\mathrm{i} \frac{\partial}{\partial \varphi}, \\
P_{0}= & -\mathrm{i}\left(-\frac{\partial}{\partial a}+\xi \frac{\partial}{\partial \xi}+z \frac{\partial}{\partial z}\right), \\
P_{1}= & -\mathrm{i}\left\{-\xi \cos \varphi \frac{\partial}{\partial a}+\frac{e^{-a}}{2}\left[-e^{-a}+\left(\xi^{2}-z^{2}+1\right) e^{a}\right] \cos \varphi \frac{\partial}{\partial \xi}\right. \\
& \left.+\xi z \cos \varphi \frac{\partial}{\partial z}+\frac{e^{-a}}{2 \xi}\left[e^{-a}+\left(\xi^{2}+z^{2}-1\right) e^{a}\right] \sin \varphi \frac{\partial}{\partial \varphi}\right\}, \\
P_{2}= & -\mathrm{i}\left\{-\xi \sin \varphi \frac{\partial}{\partial a}+\frac{e^{-a}}{2}\left[-e^{-a}+\left(\xi^{2}-z^{2}+1\right) e^{a}\right] \sin \varphi \frac{\partial}{\partial \xi}\right. \\
& \left.+\xi z \sin \varphi \frac{\partial}{\partial z}-\frac{e^{-a}}{2 \xi}\left[e^{-a}+\left(\xi^{2}+z^{2}-1\right) e^{a}\right] \cos \varphi \frac{\partial}{\partial \varphi}\right\},
\end{aligned}
$$




$$
\begin{aligned}
P_{3}= & -\mathrm{i}\left\{-z \frac{\partial}{\partial a}+z \xi \frac{\partial}{\partial \xi}+\frac{e^{-a}}{2}\left[e^{-a}-\left(z^{2}-\xi^{2}+1\right) e^{a}\right] \frac{\partial}{\partial z}\right\}, \\
N_{1}= & -\mathrm{i}\left\{\xi \cos \varphi \frac{\partial}{\partial a}+\frac{e^{-a}}{2}\left[e^{-a}+\left(z^{2}-\xi^{2}+1\right) e^{a}\right] \cos \varphi \frac{\partial}{\partial \xi}\right. \\
& \left.-z \xi \cos \varphi \frac{\partial}{\partial z}-\frac{e^{-a}}{2 \xi}\left[e^{-a}+\left(\xi^{2}+z^{2}+1\right) e^{a}\right] \sin \varphi \frac{\partial}{\partial \varphi}\right\}, \\
N_{2}= & -\mathrm{i}\left\{\xi \sin \varphi \frac{\partial}{\partial a}+\frac{e^{-a}}{2}\left[e^{-a}+\left(z^{2}-\xi^{2}+1\right) e^{a}\right] \sin \varphi \frac{\partial}{\partial \xi}\right. \\
& \left.-z \xi \sin \varphi \frac{\partial}{\partial z}+\frac{e^{-a}}{2 \xi}\left[e^{-a}+\left(\xi^{2}+z^{2}+1\right) e^{a}\right] \cos \varphi \frac{\partial}{\partial \varphi}\right\}, \\
N_{3}= & -\mathrm{i}\left\{z \frac{\partial}{\partial a}-z \xi \frac{\partial}{\partial \xi}+\frac{e^{-a}}{2}\left[e^{-a}+\left(\xi^{2}-z^{2}+1\right) e^{a}\right] \frac{\partial}{\partial z}\right\} .
\end{aligned}
$$

\section{OT-system:}

$$
\begin{aligned}
& M_{1}=-\mathrm{i}\left(y_{2} \frac{\partial}{\partial y_{3}}-y_{3} \frac{\partial}{\partial y_{2}}\right), \quad M_{2}=-\mathrm{i}\left(y_{3} \frac{\partial}{\partial y_{1}}-y_{1} \frac{\partial}{\partial y_{3}}\right), \\
& M_{3}=-\mathrm{i}\left(y_{1} \frac{\partial}{\partial y_{2}}-y_{2} \frac{\partial}{\partial y_{1}}\right), \quad P_{0}=-\mathrm{i}\left(-\frac{\partial}{\partial a}+y_{1} \frac{\partial}{\partial y_{1}}+y_{2} \frac{\partial}{\partial y_{2}}+y_{3} \frac{\partial}{\partial y_{3}}\right), \\
& P_{1}=-\mathrm{i}\left\{-y_{1} \frac{\partial}{\partial a}-\frac{e^{-a}}{2}\left[e^{-a}+\left(y^{2}-1\right) e^{a}\right] \frac{\partial}{\partial y_{1}}+y_{1}\left(y_{1} \frac{\partial}{\partial y_{1}}+y_{2} \frac{\partial}{\partial y_{2}}+y_{3} \frac{\partial}{\partial y_{3}}\right)\right\}, \\
& P_{2}=-\mathrm{i}\left\{-y_{2} \frac{\partial}{\partial a}-\frac{e^{-a}}{2}\left[e^{-a}+\left(y^{2}-1\right) e^{a}\right] \frac{\partial}{\partial y_{2}}+y_{2}\left(y_{1} \frac{\partial}{\partial y_{1}}+y_{2} \frac{\partial}{\partial y_{2}}+y_{3} \frac{\partial}{\partial y_{3}}\right)\right\}, \\
& P_{3}=-\mathrm{i}\left\{-y_{3} \frac{\partial}{\partial a}-\frac{e^{-a}}{2}\left[e^{-a}+\left(y^{2}-1\right) e^{a}\right] \frac{\partial}{\partial y_{3}}+y_{3}\left(y_{1} \frac{\partial}{\partial y_{1}}+y_{2} \frac{\partial}{\partial y_{2}}+y_{3} \frac{\partial}{\partial y_{3}}\right)\right\}, \\
& N_{1}=-\mathrm{i}\left\{y_{1} \frac{\partial}{\partial a}-\frac{e^{-a}}{2}\left[e^{-a}+\left(y^{2}+1\right) e^{a}\right] \frac{\partial}{\partial y_{1}}-y_{1}\left(y_{1} \frac{\partial}{\partial y_{1}}+y_{2} \frac{\partial}{\partial y_{2}}+y_{3} \frac{\partial}{\partial y_{3}}\right)\right\}, \\
& N_{2}=-\mathrm{i}\left\{y_{2} \frac{\partial}{\partial a}-\frac{e^{-a}}{2}\left[e^{-a}+\left(y^{2}+1\right) e^{a}\right] \frac{\partial}{\partial y_{2}}-y_{2}\left(y_{1} \frac{\partial}{\partial y_{1}}+y_{2} \frac{\partial}{\partial y_{2}}+y_{3} \frac{\partial}{\partial y_{3}}\right)\right\}, \\
& N_{3}=-\mathrm{i}\left\{y_{3} \frac{\partial}{\partial a}-\frac{e^{-a}}{2}\left[e^{-a}+\left(y^{2}+1\right) e^{a}\right] \frac{\partial}{\partial y_{3}}-y_{3}\left(y_{1} \frac{\partial}{\partial y_{1}}+y_{2} \frac{\partial}{\partial y_{2}}+y_{3} \frac{\partial}{\partial y_{3}}\right)\right\},
\end{aligned}
$$

\section{$C$-system:}

$$
\begin{aligned}
M_{1}= & -\mathrm{i}\left(-\sin \varphi \frac{\partial}{\partial \theta}-\cot \theta \cos \varphi \frac{\partial}{\partial \varphi}\right), \\
M_{2}= & -\mathrm{i}\left(\cos \varphi \frac{\partial}{\partial \theta}-\cot \theta \sin \varphi \frac{\partial}{\partial \varphi}\right), \quad M_{3}=-\mathrm{i} \frac{\partial}{\partial \varphi}, \quad P_{0}=-\mathrm{i} \frac{\partial}{\partial b}, \\
P_{1}= & -\mathrm{i}\left(-\sinh b \sin \theta \cos \varphi \frac{\partial}{\partial a}+\tanh a \cosh b \sin \theta \cos \varphi \frac{\partial}{\partial b}\right. \\
& \left.-\operatorname{coth} a \sinh b \cos \theta \cos \varphi \frac{\partial}{\partial \theta}+\operatorname{coth} a \sinh b \frac{\sin \varphi}{\sin \theta} \frac{\partial}{\partial \varphi}\right), \\
P_{2}= & -\mathrm{i}\left(-\sinh b \sin \theta \sin \varphi \frac{\partial}{\partial a}+\tanh a \cosh b \sin \theta \sin \varphi \frac{\partial}{\partial b}\right. \\
& \left.-\operatorname{coth} a \sinh b \cos \theta \sin \varphi \frac{\partial}{\partial \theta}-\operatorname{coth} a \sinh b \frac{\cos \varphi}{\sin \theta} \frac{\partial}{\partial \varphi}\right), \\
P_{3}= & -\mathrm{i}\left(-\sinh b \cos \theta \frac{\partial}{\partial a}+\tanh a \cosh b \cos \theta \frac{\partial}{\partial b}+\operatorname{coth} a \sinh b \sin \theta \frac{\partial}{\partial \theta}\right),
\end{aligned}
$$




$$
\begin{aligned}
N_{1}= & -\mathrm{i}\left(\cosh b \sin \theta \cos \varphi \frac{\partial}{\partial a}-\tanh a \sinh b \sin \theta \cos \varphi \frac{\partial}{\partial b}\right. \\
& \left.+\operatorname{coth} a \cosh b \cos \theta \cos \varphi \frac{\partial}{\partial \theta}-\operatorname{coth} a \cosh b \frac{\sin \varphi}{\sin \theta} \frac{\partial}{\partial \varphi}\right), \\
N_{2}= & -\mathrm{i}\left(\cosh b \sin \theta \sin \varphi \frac{\partial}{\partial a}-\tanh a \sinh b \sin \theta \sin \varphi \frac{\partial}{\partial b}\right. \\
& \left.+\operatorname{coth} a \cosh b \cos \theta \sin \varphi \frac{\partial}{\partial \theta}+\operatorname{coth} a \cosh b \frac{\cos \varphi}{\sin \theta} \frac{\partial}{\partial \varphi}\right), \\
N_{3}= & -\mathrm{i}\left(\cosh b \cos \theta \frac{\partial}{\partial a}-\tanh a \sinh b \cos \theta \frac{\partial}{\partial b}-\operatorname{coth} a \cosh b \sin \theta \frac{\partial}{\partial \theta}\right) .
\end{aligned}
$$

\section{$S H$-system:}

$$
\begin{aligned}
& M_{1}=-\mathrm{i}\left(\sinh b \sin \varphi \cos \Phi \frac{\partial}{\partial a}-\tanh a \cosh b \sin \varphi \cos \Phi \frac{\partial}{\partial b}\right. \\
& \left.-\tanh a \frac{\cos \varphi \cos \Phi}{\sinh b} \frac{\partial}{\partial \varphi}-\operatorname{coth} a \sinh b \sin \varphi \sin \Phi \frac{\partial}{\partial \Phi}\right), \\
& M_{2}=-\mathrm{i}\left(-\sinh b \cos \varphi \cos \Phi \frac{\partial}{\partial a}+\tanh a \cosh b \cos \varphi \cos \Phi \frac{\partial}{\partial b}\right. \\
& \left.-\tanh a \frac{\sin \varphi \cos \Phi}{\sinh b} \frac{\partial}{\partial \varphi}+\operatorname{coth} a \sinh b \cos \varphi \sin \Phi \frac{\partial}{\partial \Phi}\right), \\
& M_{3}=-\mathrm{i} \frac{\partial}{\partial \varphi}, \quad P_{3}=-\mathrm{i} \frac{\partial}{\partial \Phi}, \\
& P_{1}=-\mathrm{i}\left(\sinh b \cos \varphi \sin \Phi \frac{\partial}{\partial a}-\tanh a \cosh b \cos \varphi \sin \Phi \frac{\partial}{\partial b}\right. \\
& \left.+\tanh a \frac{\sin \varphi \sin \Phi}{\sinh b} \frac{\partial}{\partial \varphi}+\operatorname{coth} a \sinh b \cos \varphi \cos \Phi \frac{\partial}{\partial \Phi}\right), \\
& P_{2}=-\mathrm{i}\left(\sinh b \sin \varphi \sin \Phi \frac{\partial}{\partial a}-\tanh a \cosh b \sin \varphi \sin \Phi \frac{\partial}{\partial b}\right. \\
& \left.-\tanh a \frac{\cos \varphi \sin \Phi}{\sinh b} \frac{\partial}{\partial \varphi}+\operatorname{coth} a \sinh b \sin \varphi \cos \Phi \frac{\partial}{\partial \Phi}\right), \\
& N_{1}=-\mathrm{i}\left(\cos \varphi \frac{\partial}{\partial b}-\operatorname{coth} b \sin \varphi \frac{\partial}{\partial \varphi}\right), \quad N_{2}=-\mathrm{i}\left(\sin \varphi \frac{\partial}{\partial b}+\operatorname{coth} b \cos \varphi \frac{\partial}{\partial \varphi}\right), \\
& N_{3}=-\mathrm{i}\left(\cosh b \cos \Phi \frac{\partial}{\partial a}-\tanh a \sinh b \cos \Phi \frac{\partial}{\partial b}-\operatorname{coth} a \cosh b \sin \Phi \frac{\partial}{\partial \Phi}\right), \\
& P_{0}=-\mathrm{i}\left(\cosh b \sin \Phi \frac{\partial}{\partial a}-\tanh a \sinh b \sin \Phi \frac{\partial}{\partial b}+\operatorname{coth} a \cosh b \cos \Phi \frac{\partial}{\partial \Phi}\right) \text {. }
\end{aligned}
$$

Below the corresponding expressions for the infinitesimal generators $E_{i}, i=1,2,3$, in $O$-, $O C$ - and $O T$-systems will be used. They have the form

\section{$O$-system:}

$$
\begin{aligned}
& E_{1}=-\mathrm{i}\left(\sin \theta \cos \varphi \frac{\partial}{\partial r}+\frac{1}{r} \cos \theta \cos \varphi \frac{\partial}{\partial \theta}-\frac{1}{r} \frac{\sin \varphi}{\sin \theta} \frac{\partial}{\partial \varphi}\right), \\
& E_{2}=-\mathrm{i}\left(\sin \theta \sin \varphi \frac{\partial}{\partial r}+\frac{1}{r} \cos \theta \sin \varphi \frac{\partial}{\partial \theta}+\frac{1}{r} \frac{\cos \varphi}{\sin \theta} \frac{\partial}{\partial \varphi}\right), \\
& E_{3}=-\mathrm{i}\left(\cos \theta \frac{\partial}{\partial r}-\frac{1}{r} \sin \theta \frac{\partial}{\partial \theta}\right) .
\end{aligned}
$$


$O C$-system:

$$
E_{1}=-\mathrm{i}\left(\cos \varphi \frac{\partial}{\partial \xi}-\frac{\sin \varphi}{\xi} \frac{\partial}{\partial \varphi}\right), \quad E_{2}=-\mathrm{i}\left(\sin \varphi \frac{\partial}{\partial \xi}+\frac{\cos \varphi}{\xi} \frac{\partial}{\partial \varphi}\right), \quad E_{3}=-\mathrm{i} \frac{\partial}{\partial z} .
$$

\section{OT-system:}

$$
E_{1}=-\mathrm{i}\left(\frac{\partial}{\partial y_{1}}\right), \quad E_{2}=-\mathrm{i}\left(\frac{\partial}{\partial y_{2}}\right), \quad E_{3}=-\mathrm{i}\left(\frac{\partial}{\partial y_{3}}\right) .
$$

\section{Invariant operators on hyperboloid and their eigenfunctions}

\subsection{Introduction}

For each coordinate system on the hyperboloid $H_{+}^{4}$ we shall find basis functions of the space $L^{2}\left(H_{+}^{4}\right)$. They are constructed as common eigenfunctions of a full collection of commuting selfadjoint operators. Casimir operators of the group $S O_{0}(1,4)$ and of its subgroups are included into these collections. We shall see that the coordinate systems, considered above, are determined by the corresponding chains of subgroups of the group $S O_{0}(1,4)$.

As we have seen, the Casimir operators

$$
\begin{aligned}
& F=\left(P_{0}^{2}+\mathbf{N}^{2}\right)-\left(\mathbf{P}^{2}+\mathbf{M}^{2}\right), \\
& W=(\mathbf{M} \cdot \mathbf{P})^{2}-\left(P_{0} \mathbf{M}-\mathbf{P} \times \mathbf{N}\right)^{2}-(\mathbf{M} \cdot \mathbf{N})^{2}
\end{aligned}
$$

are independent invariants of the Lie algebra so $(1,4)$. The second operator vanishes on the space $L^{2}\left(H_{+}^{4}\right)$. We include the operator $F$ into full collections of commuting self-adjoint operators.

The quasi-regular representation (2.11) is reducible. Since it is unitary, it decomposes into a direct integral of irreducible unitary representations [24]. Since $H_{+}^{4} \equiv S O_{0}(1,4) / S O(4)$ and $S O(4)$ is a compact subgroup, this decomposition can be obtained from the Fourier transform and the Plancherel formula for the regular representation of the group $S O_{0}(1,4)$ [36]. The result of the decomposition of the quasi-regular representation $\pi$ of $S O_{0}(1,4)$ on $L^{2}\left(H_{+}^{4}\right)$ is the following. The representation $\pi$ on the space $L^{2}\left(H_{+}^{4}\right)$ decomposes into the direct integral of the principal unitary representations $\pi^{\sigma}, \sigma=\mathrm{i} \rho-\frac{3}{2}, 0 \leqslant \rho<\infty$, and each of these irreducible representations is contained in the decomposition only once.

The spectrum of the Casimir operator $F$ on $L^{2}\left(H_{+}^{4}\right)$ is determined by this decomposition, since on the representations $\pi^{\sigma}, \sigma=\mathrm{i} \rho-\frac{3}{2}, 0 \leqslant \rho<\infty$, this operator is multiple to the unit operator.

Since on the representation $\pi^{\sigma}$ the operator $F$ takes the value $-\sigma(\sigma+3)$, its spectrum on $L^{2}\left(H_{+}^{4}\right)$ consists of the points

$$
-(\mathrm{i} \rho-3 / 2)(\mathrm{i} \rho+3 / 2)=\rho^{2}+9 / 4, \quad 0 \leqslant \rho<\infty .
$$

The operator $-F$ on $L^{2}\left(H_{+}^{4}\right)$ coincides with the Laplace operator $\Delta\left(H_{+}^{4}\right)$ in the corresponding coordinate system (see [37]).

If we have a collection of commuting self-adjoint operators for each of coordinate systems (4.1)-(4.7), we can find their common eigenfunctions, which constitute a basis of the space $L^{2}\left(H_{+}^{4}\right)$. It is not a basis in the usual sense. It is rather a "continuous" basis (corresponding to continuous spectra of commuting operators). A strict mathematical meaning to such bases can done by using the results of [38]. Eigenfunctions of collections of self-adjoint operators will be found by means of the corresponding differential equations [9].

A differential form of commuting operators, which are contained in a collection, is found by means of differential form of the infinitesimal operators calculated above. 


\section{$5.2 \quad H$-system}

The operator $\Delta\left(H_{+}^{4}\right)=-F$ in the coordinates $a, b, \theta, \varphi$ has the form

$$
\begin{aligned}
\Delta\left(H_{+}^{4}\right)= & \frac{1}{\cosh ^{3} a} \frac{\partial}{\partial a} \cosh ^{3} a \frac{\partial}{\partial a}+\frac{1}{\cosh ^{2} a \sinh ^{2} b}\left[\frac{\partial}{\partial b} \sinh ^{2} b \frac{\partial}{\partial b}\right. \\
& \left.+\frac{1}{\sin \theta}\left(\frac{\partial}{\partial \theta} \sin \theta \frac{\partial}{\partial \theta}+\frac{1}{\sin \theta} \frac{\partial^{2}}{\partial \varphi^{2}}\right)\right] .
\end{aligned}
$$

The operators

$$
\begin{aligned}
\mathbf{N}^{2}-\mathbf{M}^{2} & \equiv N_{1}^{2}+N_{2}^{2}+N_{3}^{2}-M_{1}^{2}-M_{2}^{2}-M_{3}^{2} \\
& =-\frac{1}{\sinh ^{2} b}\left(\frac{\partial}{\partial b} \sinh ^{2} b \frac{\partial}{\partial b}+\frac{1}{\sin \theta} \frac{\partial}{\partial \theta} \sin \theta \frac{\partial}{\partial \theta}+\frac{1}{\sin ^{2} \theta} \frac{\partial^{2}}{\partial \varphi^{2}}\right), \\
\mathbf{M}^{2}=-( & \left.\frac{1}{\sin \theta} \frac{\partial}{\partial \theta} \sin \theta \frac{\partial}{\partial \theta}+\frac{1}{\sin ^{2} \theta} \frac{\partial^{2}}{\partial \varphi^{2}}\right), \quad M_{3}=-\mathrm{i} \frac{\partial}{\partial \varphi}
\end{aligned}
$$

commute with the operator $\Delta\left(H_{+}^{4}\right)$.

The operators $\Delta\left(H_{+}^{4}\right), \mathbf{N}^{2}-\mathbf{M}^{2}, \mathbf{M}^{2}, M_{3}^{2}$ are quadratic Casimir operators for the groups $S O_{0}(1,4), S O_{0}(1,3), S O(3)$ and $S O(2)$, respectively.

Let us find spectra and common eigenfunctions of self-adjoint operators $\Delta\left(H_{+}^{4}\right), \mathbf{N}^{2}-\mathbf{M}^{2}, \mathbf{M}^{2}$ and $M_{3}^{2}$ on the space $L^{2}\left(H_{+}^{4}\right)$. (If the operators (5.3) and (5.4) are considered as a product of the infinitesimal operators of the quasi-regular representation $\pi$, then they are symmetric operators and have self-adjoint extensions. If we speak about self-adjoint operators (5.3) and (5.4), then we understand that they are these self-adjoint extensions. This remark concerns also to other collections of operators considered below.)

The spectrum of the operator $\Delta\left(H_{+}^{4}\right)$ is described above. Let us discuss the spectrum of the operator $-\left(\mathbf{N}^{2}-\mathbf{M}^{2}\right)$. Its form coincides with the Laplace operator $\Delta\left(H_{+}^{3}\right)$ on the space $L^{2}\left(H_{+}^{3}\right)$ in the spherical coordinate system on $H_{+}^{3}$, where $H_{+}^{3}$ is the upper sheet of the two-sheeted hyperboloid in 4-dimensional Minkowski space, that is, $H_{+}^{3} \equiv S O_{0}(1,3) / S O(3)$. However, we have to consider $H_{+}^{3}$ as a subset of $H_{+}^{4}$. Let us take the hyperbolic coordinate system $H$ on $H_{+}^{4}$. The operator $\mathbf{N}^{2}-\mathbf{M}^{2}$ does not depend on the coordinate $a$. At each fixed $a$ a point $\left(x_{0}, x_{1}, x_{2}, x_{3}, x_{4}=\sinh a\right) \in H_{+}^{4}$ runs over the upper sheet of two-sheeted hyperboloid $x_{0}^{2}-$ $x_{1}^{2}-x_{2}^{2}-x_{3}^{2}=\cosh ^{2} a$ in the 4-dimensional space of points $\left(x_{0}, x_{1}, x_{2}, x_{3}, x_{4}\right)$. The coordinate $a$ runs over the real line $\mathbb{R}$. It is evident that there are identical 3-dimensional hyperboloids $x_{0}^{2}-x_{1}^{2}-x_{2}^{2}-x_{3}^{2}=\cosh ^{2} a$ with different values of the coordinate $x_{4}: x_{4}=\sinh a$ and $x_{4}=$ $-\sinh a$ corresponding to the points $a$ and $-a$ respectively.

The above reasoning shows that the operator $\mathbf{M}^{2}-\mathbf{N}^{2}$ leads to two Laplace operators $\Delta\left(H_{+}^{3}\right)$ on $L^{2}\left(H_{+}^{4}\right)$. One of them corresponds to the value $a \in(-\infty, 0)$ and the other to the value $a \in(0, \infty)$.

Thus, in order to find a whole spectrum of eigenvalues and the corresponding eigenfunctions of the operators (5.3)-(5.5) it is necessary to solve two systems of equations

$$
\begin{aligned}
& \Delta\left(H_{+}^{4}\right) \Phi_{\rho \nu l m}^{\varepsilon}(a, b, \theta, \varphi)=-\left(\rho^{2}+{ }^{9} / 4\right) \Phi_{\rho \nu l m}^{\varepsilon}(a, b, \theta, \varphi), \\
& \Delta\left(H_{+}^{3}\right) \Phi_{\rho \nu l m}^{\varepsilon}(a, b, \theta, \varphi)=-\left(\nu^{2}+1\right) \Phi_{\rho \nu l m}^{\varepsilon}(a, b, \theta, \varphi), \\
& \mathbf{M}^{2} \Phi_{\rho \nu l m}^{\varepsilon}(a, b, \theta, \varphi)=l(l+1) \Phi_{\rho \nu l m}^{\varepsilon}(a, b, \theta, \varphi), \\
& M_{3} \Phi_{\rho \nu l m}^{\varepsilon}(a, b, \theta, \varphi)=m \Phi_{\rho \nu l m}^{\varepsilon}(a, b, \theta, \varphi) .
\end{aligned}
$$

One system corresponds to $\varepsilon=+$ and the other to $\varepsilon=-$. 
We try to find solutions of the system (5.6) in the form of products of functions of each variables $a, b, \theta, \varphi$ :

$$
\Phi_{\rho \nu l m}^{\varepsilon}(a, b, \theta, \varphi) \equiv\langle a, b, \theta, \varphi \mid \rho, \nu, l, m\rangle^{\varepsilon}=\langle a \mid \rho, \nu\rangle^{\varepsilon}\langle b \mid \nu, l\rangle\langle\theta \mid l, m\rangle\langle\varphi \mid m\rangle .
$$

According to (5.3)-(5.5) the functions $\langle a \mid \rho, \nu\rangle^{\varepsilon},\langle b \mid \nu, l\rangle,\langle\theta \mid l, m\rangle$ and $\langle\varphi \mid m\rangle$ satisfy the equations

$$
\begin{aligned}
& \left(\frac{\partial^{2}}{\partial a^{2}}+\frac{3}{\operatorname{coth} a} \frac{\partial}{\partial a}-\frac{\nu^{2}+1}{\cosh ^{2} a}+\rho^{2}+\frac{9}{4}\right)\langle a \mid \rho, \nu\rangle^{\varepsilon}=0, \\
& \left(\frac{1}{\sinh ^{2} b} \frac{\partial}{\partial b} \sinh ^{2} b \frac{\partial}{\partial b}-\frac{l(l+1)}{\sinh ^{2} b}+\nu^{2}+1\right)\langle b \mid \nu, l\rangle=0, \\
& \left(\frac{1}{\sin \theta} \frac{\partial}{\partial \theta} \sin \theta \frac{\partial}{\partial \theta}-\frac{m^{2}}{\sin ^{2} \theta}+l(l+1)\right)\langle\theta \mid l, m\rangle=0, \\
& \left(i \frac{\partial}{\partial \varphi}+m\right)\langle\varphi \mid m\rangle=0 .
\end{aligned}
$$

The equations (5.9)-(5.11) give a system of equations for eigenvalues and eigenfunctions for the collection of self-adjoint operators on the space $L^{2}\left(H_{+}^{3}\right)$. A solution of this problem is given in [9]. Therefore, solutions of the system of equations (5.8)-(5.11) are the functions

$$
\begin{aligned}
& \langle a \mid \rho, \nu\rangle^{\varepsilon}=(\cosh a)^{-3 / 2} P_{-1 / 2+i \nu}^{-i \rho}(\varepsilon \tanh a), \quad \varepsilon= \pm, \quad 0 \leqslant \rho<\infty, \\
& \langle b \mid \nu, l\rangle=(\sinh b)^{-1 / 2} P_{-1 / 2+i \nu}^{-1 / 2-l}(\cosh b), \quad 0 \leqslant \nu<\infty, \\
& \langle\theta \mid l, m\rangle=P_{l}^{m}(\cos \theta), \quad\langle\varphi \mid m\rangle=e^{i m \varphi}, \quad l=0,1,2, \ldots, \quad-l \leqslant m \leqslant l,
\end{aligned}
$$

where $P_{\nu}^{\mu}(z)$ are associated Legendre functions and $P_{l}^{m}(\cos \theta)$ are associated Legendre functions on the cut. Thus, the functions

$$
\begin{aligned}
&\langle a, b, \theta, \varphi \mid \rho, \nu, l, m\rangle^{\varepsilon}=(\cosh a)^{-3 / 2}(\sinh b)^{-1 / 2} P_{-1 / 2+i \nu}^{-i \rho}(\varepsilon \tanh a) \\
& \times P_{-1 / 2+i \nu}^{-1 / 2-l}(\cosh b) P_{l}^{m}(\cos \theta) e^{i m \varphi}, \\
& \varepsilon= \pm, \quad 0 \leqslant \rho, \nu<\infty, \quad l=0,1,2, \ldots, \quad-l \leqslant m \leqslant l,
\end{aligned}
$$

constitute a basis (not normed) of the space $L^{2}\left(H_{+}^{4}\right)$ in the $H$-coordinate system. This basis corresponds to the chain of subgroups

$$
S O_{0}(1,4) \supset S O_{0}(1,3) \supset S O(3) \supset S O(2) .
$$

Remark. The operators (5.3)-(5.5) do not constitute a full collection of commuting self-adjoint operators in the space $L^{2}\left(H_{+}^{4}\right)$. For obtaining a full collection of such operators we have to add to the collection (5.3)-(5.5) an operator, which separates eigenfunctions with different values of $\varepsilon$. However, we have found the whole system of eigenfunctions without use of this operator. Collections of self-adjoint operators, considered below for other coordinate systems, will constitute a full collections of self-adjoint operators.

\section{$5.3 \quad O$-system}

In this coordinate system, the Laplace operator is of the form

$$
\Delta\left(H_{+}^{4}\right)=\frac{\partial^{2}}{\partial a^{2}}+3 \frac{\partial}{\partial a}+e^{-2 a}\left[\frac{\partial^{2}}{\partial r^{2}}+\frac{2}{r} \frac{\partial}{\partial r}+\frac{1}{r^{2}}\left(\frac{\partial^{2}}{\partial \theta^{2}}+\cot \theta \frac{\partial}{\partial \theta}+\frac{1}{\sin ^{2} \theta} \frac{\partial^{2}}{\partial \varphi^{2}}\right)\right] .
$$


If we introduce the variable $b=e^{-a}$, then $\Delta\left(H_{+}^{4}\right)$ can be written in the form

$$
\Delta\left(H_{+}^{4}\right)=b^{2} \frac{\partial^{2}}{\partial b^{2}}-2 b \frac{\partial}{\partial b}+b^{2}\left[\frac{\partial^{2}}{\partial r^{2}}+\frac{2}{r} \frac{\partial}{\partial r}+\frac{1}{r^{2}}\left(\frac{\partial^{2}}{\partial \theta^{2}}+\cot \theta \frac{\partial}{\partial \theta}+\frac{1}{\sin ^{2} \theta} \frac{\partial^{2}}{\partial \varphi^{2}}\right)\right] .
$$

The operators

$$
\begin{aligned}
& -\mathbf{E}^{2} \equiv \Delta\left(\mathbb{R}^{3}\right)=\frac{\partial^{2}}{\partial r^{2}}+\frac{2}{r} \frac{\partial}{\partial r}+\frac{1}{r^{2}}\left(\frac{\partial^{2}}{\partial \theta^{2}}+\cot \theta \frac{\partial}{\partial \theta}+\frac{1}{\sin ^{2} \theta} \frac{\partial^{2}}{\partial \varphi^{2}}\right), \\
& \mathbf{M}^{2}=-\left(\frac{1}{\sin \theta} \frac{\partial}{\partial \theta} \sin \theta \frac{\partial}{\partial \theta}+\frac{1}{\sin ^{2} \theta} \frac{\partial^{2}}{\partial \varphi^{2}}\right), \quad M_{3}=-i \frac{\partial}{\partial \varphi}
\end{aligned}
$$

commute with the operator (5.13). The operators $\mathbf{E}^{2}, \mathbf{M}^{2}$ and $M_{3}^{2}$ are Casimir operators for the subgroups $I S O(3), S O(3)$ and $S O(2)$, respectively. Therefore, a basis of the space $L^{2}\left(H_{+}^{4}\right)$ in the $O$-coordinate system, which will be found below, corresponds to the chain of subgroups

$$
S O_{0}(1,4) \supset I S O(3) \supset S O(3) \supset S O(2) .
$$

The operator $\Delta\left(\mathbb{R}^{3}\right)$ coincides with the 3-dimensional Laplace operator on the Euclidean space $\mathbb{R}^{3}$ in the spherical coordinate system. The space $\mathbb{R}^{3}$ is obtained from $H_{+}^{4}$ by cutting this hyperboloid by the hyperplane $e^{-a}=$ const. At each fixed $a$, functions $f(a, r, \theta, \varphi)$ of $L^{2}\left(H_{+}^{4}\right)$ lead to functions of $L^{2}\left(\mathbb{R}^{3}\right)$. This means that the space $L^{2}\left(H_{+}^{4}\right)$ is a direct integral over values of $a$ of the spaces $L_{a}^{2}\left(\mathbb{R}^{3}\right) \equiv L^{2}\left(\mathbb{R}^{3}\right)$. Eigenfunctions and eigenvalues of the operator $\Delta\left(\mathbb{R}^{3}\right)$ on the space $L^{2}\left(\mathbb{R}^{3}\right)$ are known. Since $\mathbb{R}^{3} \equiv I S O(3) / S O(3)$, they can be obtained, for example, from the Fourier transform and Plancherel formula for the regular representation of the group $\operatorname{ISO}(3)$.

The system of differential equations for eigenfunctions and eigenvalues of the operators (5.13)-(5.15) in the $O$-coordinate system is of the form

$$
\begin{aligned}
& \Delta\left(H_{+}^{4}\right) \Phi_{l m}^{\rho \kappa}(b, r, \theta, \varphi)=-\left(\rho^{2}+9 / 4\right) \Phi_{l m}^{\rho \kappa}(b, r, \theta, \varphi), \\
& \Delta\left(\mathbb{R}^{3}\right) \Phi_{l m}^{\rho \kappa}(b, r, \theta, \varphi)=-\kappa^{2} \Phi_{l m}^{\rho \kappa}(b, r, \theta, \varphi), \\
& \mathbf{M}^{2} \Phi_{l m}^{\rho \kappa}(b, r, \theta, \varphi)=l(l+1) \Phi_{l m}^{\rho \kappa}(b, r, \theta, \varphi), \\
& M_{3} \Phi_{l m}^{\rho \kappa}(b, r, \theta, \varphi)=m \Phi_{l m}^{\rho \kappa}(b, r, \theta, \varphi) .
\end{aligned}
$$

We try to find solutions in the form of separated variables:

$$
\Phi_{l m}^{\rho \kappa}(b, r, \theta, \varphi) \equiv\langle b, r, \theta, \varphi \mid \rho, \kappa, l, m\rangle=\langle b \mid \rho, \kappa\rangle\langle r \mid \kappa, l\rangle\langle\theta \mid l, m\rangle\langle\varphi \mid m\rangle .
$$

For these solutions the system (5.17) take the form

$$
\begin{aligned}
& \left(\frac{\partial^{2}}{\partial b^{2}}-\frac{2}{b} \frac{\partial}{\partial b}+\left(-\kappa^{2}+\frac{\rho^{2}+9 / 4}{b^{2}}\right)\right)\langle b \mid \rho, \kappa\rangle=0, \quad 0 \leqslant \rho<\infty, \\
& \left(\frac{\partial^{2}}{\partial r^{2}}+\frac{2}{r} \frac{\partial}{\partial r}+\kappa^{2}-\frac{l(l+1)}{r^{2}}\right)\langle r \mid \kappa, l\rangle=0, \quad 0 \leqslant \kappa<\infty, \\
& \left(\frac{1}{\sin \theta} \frac{\partial}{\partial \theta} \sin \theta \frac{\partial}{\partial \theta}-\frac{m^{2}}{\sin ^{2} \theta}-l(l+1)\right)\langle\theta \mid l, m\rangle=0, \quad l=0,1,2, \ldots, \\
& \left(\mathrm{i} \frac{\partial}{\partial \varphi}+m\right)\langle\varphi \mid m\rangle=0, \quad-l \leqslant m \leqslant l .
\end{aligned}
$$

The functions

$$
\langle b \mid \rho, \kappa\rangle=(\kappa b)^{3 / 2} K_{\mathrm{i} \rho}(\kappa b), \quad\langle r \mid \kappa, l\rangle=(\kappa r)^{-1 / 2} J_{l+1 / 2}(\kappa r),
$$




$$
\langle\theta \mid l, m\rangle=P_{l}^{m}(\cos \theta), \quad\langle\varphi \mid m\rangle=e^{\mathrm{i} m \varphi},
$$

are solutions of the equations (5.18)-(5.21), where $K_{\mathrm{i} \rho}(\kappa b)$ is the Macdonald function and $J_{l+1 / 2}(\kappa r)$ is the Bessel function.

Thus, the functions

$$
\begin{aligned}
& \langle b, r, \theta, \varphi \mid \rho, \kappa, l, m\rangle=(\kappa b)^{3 / 2}(\kappa r)^{-1 / 2} K_{\mathrm{i} \rho}(\kappa b) J_{l+1 / 2}(\kappa r) P_{l}^{m}(\cos \theta) e^{\mathrm{i} m \varphi} \\
& 0 \leqslant \rho, \kappa<\infty, \quad l=0,1,2, \ldots, \quad-l \leqslant m \leqslant l, \quad b=e^{-a},
\end{aligned}
$$

constitute a basis of the space $L^{2}\left(H_{+}^{4}\right)$ in the $O$-system of coordinates.

\section{$5.4 O C$-system}

In the $O C$-coordinate system, the Laplace operator is of the form

$$
\Delta\left(H_{+}^{4}\right)=b^{2} \frac{\partial^{2}}{\partial b^{2}}-2 b \frac{\partial}{\partial b}+b^{2}\left(\frac{\partial^{2}}{\partial \xi^{2}}+\frac{1}{\xi} \frac{\partial}{\partial \xi}+\frac{1}{\xi^{2}} \frac{\partial^{2}}{\partial \varphi^{2}}+\frac{\partial^{2}}{\partial z^{2}}\right) .
$$

The operators

$$
\begin{aligned}
& \mathbf{E}^{2}=-\Delta\left(\mathbb{R}^{3}\right)=-\left(\frac{\partial^{2}}{\partial \xi^{2}}+\frac{1}{\xi} \frac{\partial}{\partial \xi}+\frac{1}{\xi^{2}} \frac{\partial^{2}}{\partial \varphi^{2}}+\frac{\partial^{2}}{\partial z^{2}}\right) \\
& E_{3}=-i \frac{\partial}{\partial z}, \quad M_{3}=-i \frac{\partial}{\partial \varphi}, \\
& \tilde{\mathbf{E}}^{2}=E_{1}^{2}+E_{2}^{2}=-\Delta\left(\mathbb{R}^{2}\right)=-\left(\frac{\partial^{2}}{\partial \xi^{2}}+\frac{1}{\xi} \frac{\partial}{\partial \xi}+\frac{1}{\xi^{2}} \frac{\partial^{2}}{\partial \varphi^{2}}\right)
\end{aligned}
$$

commute with the operator (5.23). The operator $\Delta\left(\mathbb{R}^{3}\right)$ is the Laplace operator on the space $\mathbb{R}^{3}$ in the cylindric coordinates. The operators $\tilde{\mathbf{E}}^{2}$ and $E_{3}^{2}$ are Casimir operators of the group $I S O(2) \otimes T_{\perp}$, and the operators $E_{3}^{2}$ and $M_{3}^{2}$ are Casimir operators of $S O(2) \otimes T_{\perp}$. Eigenvalues of the operators $(5.24)-(5.26)$ in $L^{2}\left(H_{+}^{4}\right)$ are

$$
\begin{aligned}
& \nu\left(\mathbf{E}^{2}\right)=\kappa^{2}, \quad \nu\left(E_{3}\right)=q, \quad \nu\left(M_{3}\right)=m, \quad \nu\left(\tilde{\mathbf{E}}^{2}\right)=\eta^{2}, \\
& \kappa^{2}=q^{2}+\eta^{2}, \quad-\infty<q<\infty, \quad 0 \leqslant \eta<\infty, \quad m=0, \pm 1, \pm 2, \ldots
\end{aligned}
$$

We have the following system of differential equations for eigenfunctions:

$$
\begin{aligned}
& \Delta\left(H_{+}^{4}\right) \Phi_{\rho \eta q}^{m}(b, \xi, z, \varphi)=-\left(\rho^{2}+9 / 4\right) \Phi_{\rho \eta q}^{m}(b, \xi, z, \varphi), \quad 0 \leqslant \rho<\infty, \\
& \Delta\left(\mathbb{R}^{2}\right) \Phi_{\rho \eta q}^{m}(b, \xi, z, \varphi)=-\eta^{2} \Phi_{\rho \eta q}^{m}(b, \xi, z, \varphi), \quad 0 \leqslant \eta<\infty, \\
& E_{3} \Phi_{\rho \eta q}^{m}(b, \xi, z, \varphi)=q \Phi_{\rho \eta q}^{m}(b, \xi, z, \varphi), \quad-\infty<q<\infty, \\
& M_{3} \Phi_{\rho \eta q}^{m}(b, \xi, z, \varphi)=m \Phi_{\rho \eta q}^{m}(b, \xi, z, \varphi), \quad m=0, \pm 1, \pm 2, \ldots
\end{aligned}
$$

Solutions of this system can be represented in the form of separated variables:

$$
\Phi_{\rho \eta q}^{m}(b, \xi, z, \varphi) \equiv\langle b, \xi, z, \varphi \mid \rho, \eta, q, m\rangle=\langle b \mid \rho, \kappa\rangle\langle\xi \mid \eta, m\rangle\langle z \mid q\rangle\langle\varphi \mid m\rangle,
$$

where $\kappa^{2}=\eta^{2}+q^{2}$. After separation of variables the system (5.27) takes the form

$$
\begin{aligned}
& \left(\frac{\partial^{2}}{\partial b^{2}}-\frac{2}{b} \frac{\partial}{\partial b}+\frac{\rho^{2}+9 / 4}{b^{2}}-\kappa^{2}\right)\langle b \mid \rho, \kappa\rangle=0, \\
& \left(\frac{\partial^{2}}{\partial \xi^{2}}+\frac{1}{\xi} \frac{\partial}{\partial \xi}-\frac{m^{2}}{\xi^{2}}+\eta^{2}\right)\langle\xi \mid \eta, m\rangle=0
\end{aligned}
$$




$$
\left(i \frac{\partial}{\partial z}+q\right)\langle q \mid z\rangle=0, \quad\left(\mathrm{i} \frac{\partial}{\partial \varphi}+m\right)\langle\varphi \mid m\rangle=0 .
$$

Solutions of these equations are of the form

$$
\begin{aligned}
& \langle b \mid \rho, \kappa\rangle=(\kappa b)^{3 / 2} K_{\mathrm{i} \rho}(\kappa b), \quad\langle\xi \mid \eta, m\rangle=J_{m}(\eta \xi), \quad \eta^{2}=\kappa^{2}-q^{2}, \\
& \langle z \mid q\rangle=e^{\mathrm{i} q z}, \quad\langle\varphi \mid m\rangle=e^{\mathrm{i} m \varphi} .
\end{aligned}
$$

Therefore, the functions

$$
\begin{aligned}
& \langle b, \xi, z, \varphi \mid \rho, \eta, q, m\rangle=(\kappa b)^{3 / 2} K_{\mathrm{i} \rho}(\kappa b) J_{m}(\eta \xi) e^{\mathrm{i} q z} e^{\mathrm{i} m \varphi}, \\
& \eta^{2}=\kappa^{2}-q^{2}, \quad 0 \leqslant \rho, \eta<\infty, \quad-\infty<q<\infty, \quad m=0, \pm 1, \pm 2, \ldots,
\end{aligned}
$$

constitute a basis of the space $L^{2}\left(H_{+}^{4}\right)$ in the $O C$-coordinate system. This basis corresponds to the chain of subgroups

$$
S O_{0}(1,4) \supset I S O(3) \supset I S O(2) \otimes T_{\perp} \supset S O(2) \otimes T_{\perp} .
$$

\section{$5.5 O T$-system}

In this coordinate system

$$
\Delta\left(H_{+}^{4}\right)=b^{2} \frac{\partial^{2}}{\partial b^{2}}-2 b \frac{\partial}{\partial b}+b^{2}\left(\frac{\partial^{2}}{\partial y_{1}^{2}}+\frac{\partial^{2}}{\partial y_{2}^{2}}+\frac{\partial^{2}}{\partial y_{3}^{2}}\right), \quad b=e^{-a} .
$$

The operators

$$
\mathbf{E}^{2}=\Delta\left(\mathbb{R}^{3}\right)=-\left(\frac{\partial^{2}}{\partial y_{1}^{2}}+\frac{\partial^{2}}{\partial y_{2}^{2}}+\frac{\partial^{2}}{\partial y_{3}^{2}}\right), \quad E_{j}=-i \frac{\partial}{\partial y_{j}}, \quad j=1,2,3,
$$

commute with $\Delta\left(H_{+}^{4}\right)$. The operator $\Delta\left(\mathbb{R}^{3}\right)$ is an invariant for the group $I S O(3)$. It is the Laplace operator on the 3-dimensional Euclidean space in the homogeneous coordinates $y_{1}, y_{2}, y_{3}$. The $I S O(3)$ is a motion group of this Euclidean space. Eigenvalues of the operators (5.33) are

$$
\nu\left(\mathbf{E}^{2}\right)=\kappa^{2}, \quad \nu\left(E_{j}\right)=\kappa_{j}, \quad-\infty<\kappa_{j}<\infty, \quad 0 \leqslant \kappa<\infty, \quad \kappa_{1}^{2}+\kappa_{2}^{2}+\kappa_{3}^{2}=\kappa^{2} .
$$

The spectrum of the collection of operators (5.32) and (5.33) is simple. We represent eigenfunctions $\langle b, \mathbf{y} \mid \rho, \boldsymbol{\kappa}\rangle, \boldsymbol{\kappa}=\left(\kappa_{1}, \kappa_{2}, \kappa_{3}\right), \mathbf{y}=\left(y_{1}, y_{2}, y_{3},\right)$, of this collection of operators in the form

$$
\langle b, \mathbf{y} \mid \rho, \boldsymbol{\kappa}\rangle=\langle b \mid \rho, \kappa\rangle\langle\mathbf{y} \mid \boldsymbol{\kappa}\rangle, \quad \kappa=|\boldsymbol{\kappa}| .
$$

For $\langle b \mid \rho, \kappa\rangle$ and $\langle\mathbf{y} \mid \boldsymbol{\kappa}\rangle$ we have the equations

$$
\left(\frac{\partial^{2}}{\partial b^{2}}-\frac{2}{b} \frac{\partial}{\partial b}+\frac{\rho^{2}+9 / 4}{b^{2}}-\kappa^{2}\right)\langle b \mid \rho, \kappa\rangle=0, \quad\left(\mathrm{i} \frac{\partial}{\partial \mathbf{y}}+\boldsymbol{\kappa}\right)\langle\mathbf{y} \mid \boldsymbol{\kappa}\rangle=0 .
$$

Solutions of these equations are

$$
\langle b \mid \rho, \kappa\rangle=(\kappa b)^{3 / 2} K_{\mathrm{i} \rho}(\kappa b), \quad\langle\mathbf{y} \mid \boldsymbol{\kappa}\rangle=e^{\mathrm{i} \kappa \mathbf{y}}, \quad b=e^{-a},
$$

where $\boldsymbol{\kappa} \mathbf{y}=\kappa_{1} y_{1}+\kappa_{2} y_{2}+\kappa_{3} y_{3}$. Therefore, the functions

$$
\begin{aligned}
& \langle b, \mathbf{y} \mid \rho, \boldsymbol{\kappa}\rangle=(\kappa b)^{3 / 2} K_{\mathrm{i} \rho}(\kappa b) e^{\mathrm{i} \kappa \mathbf{y}}, \quad b=e^{-a}, \\
& \kappa^{2}=\kappa_{1}^{2}+\kappa_{2}^{2}+\kappa_{3}^{2}, \quad 0 \leqslant \rho, \kappa<\infty, \quad-\infty<\kappa_{j}<\infty,
\end{aligned}
$$

constitute a basis of the space $L^{2}\left(H_{+}^{4}\right)$ in the $O T$-coordinate system. 


\section{$5.6 C$-system}

In this coordinate system the Laplace operator on $H_{+}^{4}$ is of the form

$$
\begin{aligned}
\Delta\left(H_{+}^{4}\right)= & \frac{1}{\cosh a \sinh ^{2} a} \frac{\partial}{\partial a} \cosh a \sinh ^{2} a \frac{\partial}{\partial a}+\frac{1}{\cosh ^{2} a} \frac{\partial^{2}}{\partial b^{2}} \\
& +\frac{1}{\sinh ^{2} a}\left(\frac{1}{\sin \theta} \frac{\partial}{\partial \theta} \sin \theta \frac{\partial}{\partial \theta}+\frac{1}{\sin ^{2} \theta} \frac{\partial^{2}}{\partial \varphi^{2}}\right) .
\end{aligned}
$$

The operators

$$
\begin{aligned}
& \mathbf{M}^{2}=-\frac{1}{\sin \theta} \frac{\partial}{\partial \theta} \sin \theta \frac{\partial}{\partial \theta}-\frac{1}{\sin ^{2} \theta} \frac{\partial^{2}}{\partial \varphi^{2}}, \\
& M_{3}=-\mathrm{i} \frac{\partial}{\partial \varphi}, \quad P_{0}=-\mathrm{i} \frac{\partial}{\partial b}
\end{aligned}
$$

commute with $\Delta\left(H_{+}^{4}\right)$. They are invariants of the subgroups $S O(3), S O(2)$ and $S O_{0}(1,1)$ of the group $S O_{0}(1,1) \otimes S O(3)$. Their eigenvalues are

$$
\begin{array}{lr}
\nu\left(\mathbf{M}^{2}\right)=l(l+1), \quad \nu\left(M_{3}\right)=m, & \nu\left(P_{0}\right)=\tau, \\
-\infty<\tau<\infty, & l=0,1,2, \ldots,
\end{array}
$$

We try to find eigenfunctions of the operators (5.36) and (5.37) in the form

$$
\langle a, b, \theta, \varphi \mid \rho, \tau, l, m\rangle=\langle a \mid \rho, \tau, l\rangle\langle b \mid \tau\rangle\langle\theta \mid l, m\rangle\langle\varphi \mid m\rangle .
$$

The functions on the right hand side satisfy the equations

$$
\begin{aligned}
& \left\{\frac{\partial^{2}}{\partial a^{2}}+(\tanh a+2 \operatorname{coth} a) \frac{\partial}{\partial a}+\rho^{2}+\frac{9}{4}-\frac{\tau^{2}}{\cosh ^{2} a}-\frac{l(l+1)}{\sinh ^{2} a}\right\}\langle a \mid \rho, \tau, l\rangle=0, \\
& \left\{\frac{1}{\sin \theta} \frac{\partial}{\partial \theta} \sin \theta \frac{\partial}{\partial \theta}-\frac{m^{2}}{\sin ^{2} \theta}+l(l+1)\right\}\langle\theta \mid l, m\rangle=0, \\
& \left(i \frac{\partial}{\partial b}+\tau\right)\langle b \mid \tau\rangle=0, \quad\left(i \frac{\partial}{\partial \varphi}+m\right)\langle\varphi \mid m\rangle=0 .
\end{aligned}
$$

These equations have solutions

$$
\begin{aligned}
& \langle a \mid \rho, \tau, l\rangle=\tanh ^{l} a(\cosh a)^{\mathrm{i} \rho-3 / 2}{ }_{2} F_{1}\left(\frac{l-\mathrm{i} \rho+\mathrm{i} \tau+3 / 2}{2}, \frac{l-\mathrm{i} \rho-\mathrm{i} \tau+3 / 2}{2} ; l+\frac{3}{2} ; \tanh ^{2} a\right), \\
& \langle\theta \mid l, m\rangle=P_{l}^{m}(\cos \theta), \quad\langle b \mid \tau\rangle=e^{\mathrm{i} \tau b}, \quad\langle\varphi \mid m\rangle=e^{\mathrm{i} m \varphi},
\end{aligned}
$$

where ${ }_{2} F_{1}$ is a Gauss hypergeometric function. Thus, the functions

$$
\begin{aligned}
& \langle a, b, \theta, \varphi \mid \rho, \tau, l, m\rangle=\tanh ^{l} a(\cosh a)^{\mathrm{i} \rho-3 / 2} \\
& \quad \times{ }_{2} F_{1}\left(\frac{l-\mathrm{i} \rho+\mathrm{i} \tau+3 / 2}{2}, \frac{l-\mathrm{i} \rho-\mathrm{i} \tau+3 / 2}{2} ; l+\frac{3}{2} ; \tanh ^{2} a\right) P_{l}^{m}(\cos \theta) e^{\mathrm{i} \tau b} e^{\mathrm{i} m \varphi}, \\
& 0 \leqslant \rho<\infty, \quad-\infty<\tau<\infty, \quad l=0,1,2, \ldots, \quad-l \leqslant m \leqslant l,
\end{aligned}
$$

constitute a basis of the space $L^{2}\left(H_{+}^{4}\right)$ in the $C$-coordinate system. This basis corresponds to the chain of subgroups

$$
S O_{0}(1,4) \supset S O_{0}(1,1) \otimes S O(3) \supset S O(2) .
$$




\section{$5.7 \quad S H$-system}

In this coordinate system the Laplace operator on $H_{+}^{4}$ is of the form

$$
\begin{aligned}
\Delta\left(H_{+}^{4}\right)= & \frac{1}{\cosh ^{2} a \sinh a} \frac{\partial}{\partial a} \cosh ^{2} a \sinh a \frac{\partial}{\partial a}+\frac{1}{\sinh ^{2} a} \frac{\partial^{2}}{\partial \Phi^{2}} \\
& +\frac{1}{\cosh ^{2} a}\left(\frac{\partial^{2}}{\partial b^{2}}+\operatorname{coth} b \frac{\partial}{\partial b}+\frac{1}{\sinh ^{2} b} \frac{\partial^{2}}{\partial \varphi^{2}}\right) .
\end{aligned}
$$

The operators

$$
\begin{aligned}
& M_{3}^{2}-N_{1}^{2}-N_{2}^{2}=\Delta\left(H_{+}^{2}\right)=\frac{\partial^{2}}{\partial b^{2}}+\operatorname{coth} b \frac{\partial}{\partial b}+\frac{1}{\sinh ^{2} b} \frac{\partial^{2}}{\partial \varphi^{2}}, \\
& M_{3}=-\mathrm{i} \frac{\partial}{\partial \varphi}, \quad P_{3}=-\mathrm{i} \frac{\partial}{\partial \Phi}
\end{aligned}
$$

commute with the operator $\Delta\left(H_{+}^{4}\right)$. They are invariants of the subgroups $S O_{0}(1,2), S O(2)$ and $S O^{\prime}(2)$ of the group $S O_{0}(1,2) \otimes S O^{\prime}(2)$, where $S O^{\prime}(2) \backsim S O(2)$. The subgroup $S O^{\prime}(2)$ is generated by the generator $P_{3}$. The operator $\Delta\left(H_{+}^{2}\right)$ is the Laplace operator on $H_{+}^{2} \equiv$ $S O_{0}(1,2) / S O(2)$ in the spherical coordinates. Eigenvalues of the above operators are

$$
\begin{array}{ll}
\nu\left(\Delta\left(H_{+}^{4}\right)\right)=-\left(\rho^{2}+9 / 4\right), & \nu\left(\Delta\left(H_{+}^{2}\right)\right)=-\left(\omega^{2}+1 / 4\right), \quad \nu\left(M_{3}\right)=m, \quad \nu\left(P_{3}\right)=m^{\prime}, \\
0 \leqslant \rho<\infty, \quad 0 \leqslant \omega<\infty, & m, m^{\prime}=0, \pm 1, \pm 2, \ldots
\end{array}
$$

We represent solutions of the system of equations (5.44) and (5.45) in the form

$$
\left\langle a, b, \Phi, \varphi \mid \rho, \omega, m^{\prime}, m\right\rangle=\langle a \mid \rho, \omega\rangle\langle b \mid \omega, m\rangle\left\langle\Phi \mid m^{\prime}\right\rangle\langle\varphi \mid m\rangle .
$$

Then this separation of variables leads to the equations

$$
\begin{aligned}
& \left\{\frac{\partial^{2}}{\partial a^{2}}+(2 \tanh a+\operatorname{coth} a) \frac{\partial}{\partial a}-\frac{\omega^{2}+1 / 4}{\cosh ^{2} a}-\frac{m^{\prime 2}}{\sinh ^{2} a}+\rho^{2}+\frac{9}{4}\right\}\left\langle a \mid \rho, \omega, m^{\prime}\right\rangle=0, \\
& \left(\frac{\partial^{2}}{\partial b^{2}}+\operatorname{coth} b \frac{\partial}{\partial b}-\frac{m^{2}}{\sinh ^{2} b}+\omega^{2}+1 / 4\right)\langle b \mid \omega, m\rangle=0, \\
& \left(\mathrm{i} \frac{\partial}{\partial \Phi}+m^{\prime}\right)\left\langle\Phi \mid m^{\prime}\right\rangle=0, \quad\left(\mathrm{i} \frac{\partial}{\partial \varphi}+m\right)\langle\varphi \mid m\rangle=0 .
\end{aligned}
$$

Solutions of these equations are the functions

$$
\begin{aligned}
&\left\langle a \mid \rho, \omega, m^{\prime}\right\rangle=(\tanh a)^{m^{\prime}(\cosh a)^{\mathrm{i} \rho-3 / 2}} \\
& \times{ }_{2} F_{1}\left(\frac{m^{\prime}-\mathrm{i} \rho+\mathrm{i} \omega+1}{2}, \frac{m^{\prime}-\mathrm{i} \rho-\mathrm{i} \omega+1}{2} ; m^{\prime}+1 ; \tanh ^{2} a\right), \\
&\langle b \mid \omega, m\rangle=P_{\mathrm{i} \omega-1 / 2}^{m}(\cosh b), \quad\left\langle\Phi \mid m^{\prime}\right\rangle=e^{\mathrm{i} m^{\prime} \Phi}, \quad\langle\varphi \mid m\rangle=e^{\mathrm{i} m \varphi} .
\end{aligned}
$$

Thus, the functions

$$
\begin{aligned}
\left\langle a, b, \Phi, \varphi \mid \rho, \omega, m^{\prime}, m\right\rangle= & (\tanh a)^{m^{\prime}}(\cosh a)^{i \rho-3 / 2} P_{\mathrm{i} \omega-1 / 2}^{m}(\cosh b) e^{\mathrm{i} m^{\prime} \Phi} e^{\mathrm{i} m \varphi} \\
& \times{ }_{2} F_{1}\left(\frac{m^{\prime}-\mathrm{i} \rho+\mathrm{i} \omega+1}{2}, \frac{m^{\prime}-\mathrm{i} \rho-\mathrm{i} \omega+1}{2} ; m^{\prime}+1 ; \tanh ^{2} a\right), \\
0 \leqslant \rho, \omega<\infty, \quad m, m^{\prime}= & 0, \pm 1, \pm 2, \ldots,
\end{aligned}
$$

constitute a basis of the space $L^{2}\left(H_{+}^{4}\right)$ in the $S H$-coordinate system. This basis corresponds to the chain of subgroups

$$
S O_{0}(1,4) \supset S O_{0}(1,2) \otimes S O^{\prime}(2) \supset S O(2) .
$$




\section{$5.8 S$-system}

The problem of finding basis functions of the Hilbert space $L^{2}\left(H_{+}^{4}\right)$ in the spherical coordinates is solved in [25] (see also [3]). The Laplace operator in this coordinate system has the form

$$
\begin{aligned}
\Delta\left(H_{+}^{4}\right)= & \frac{1}{\sinh ^{3} a} \frac{\partial}{\partial a} \sinh ^{3} a \frac{\partial}{\partial a} \\
& +\frac{1}{\sin ^{2} \beta}\left(\frac{\partial}{\partial \beta} \sin ^{2} \beta \frac{\partial}{\partial \beta}+\frac{1}{\sin \theta} \frac{\partial}{\partial \theta} \sin \theta \frac{\partial}{\partial \theta}+\frac{1}{\sin ^{2} \theta} \frac{\partial^{2}}{\partial \varphi^{2}}\right) .
\end{aligned}
$$

The following operators commute with $\Delta_{L}\left(H_{+}^{4}\right)$ :

$$
\begin{aligned}
\mathbf{J}^{2} & \equiv \mathbf{M}^{2}+\mathbf{P}^{2}=-\Delta\left(S^{3}\right) \\
& =-\frac{1}{\sin ^{2} \beta}\left(\frac{\partial}{\partial \beta} \sin ^{2} \beta \frac{\partial}{\partial \beta}+\frac{1}{\sin \theta} \frac{\partial}{\partial \theta} \sin \theta \frac{\partial}{\partial \theta}+\frac{1}{\sin ^{2} \theta} \frac{\partial^{2}}{\partial \varphi^{2}}\right), \\
\mathbf{M}^{2} & =-\left(\frac{1}{\sin \theta} \frac{\partial}{\partial \theta} \sin \theta \frac{\partial}{\partial \theta}+\frac{1}{\sin ^{2} \theta} \frac{\partial^{2}}{\partial \varphi^{2}}\right), \quad M_{3}=-\mathrm{i} \frac{\partial}{\partial \varphi} .
\end{aligned}
$$

They are invariants of the groups $S O(4), S O(3)$ and $S O(2)$, respectively. The operator $\Delta\left(S^{3}\right)$ in (5.51) is the Laplace operator on the sphere $S^{3}$ in the 3-dimensional Euclidean space. Eigenvalues $\nu(I)$ of the operators (5.50)-(5.52) are

$$
\begin{aligned}
& \nu\left(\Delta\left(H_{+}^{4}\right)\right)=-\left(\rho^{2}+9 / 4\right), \quad \nu\left(\mathbf{J}^{2}\right)=j(j+2), \quad \nu\left(\mathbf{M}^{2}\right)=l(l+1), \quad \nu\left(M_{3}\right)=m, \\
& 0 \leqslant \rho<\infty, \quad j=0,1,2, \ldots, \quad 0 \leqslant l \leqslant j, \quad-l \leqslant m \leqslant l,
\end{aligned}
$$

and the spectrum of this collection of operators is simple. Eigenfunctions of the collection $(5.50)-(5.52)$ are $[25]$

$$
\langle a, \beta, \theta, \varphi \mid \rho, j, l, m\rangle=\sinh ^{-1} a \sin ^{-1 / 2} \beta P_{\mathrm{i} \rho-1 / 2}^{-j-1}(\cosh a) P_{j+1 / 2}^{-l-1 / 2}(\cos \beta) P_{l}^{m}(\cos \theta) e^{\mathrm{i} m \varphi} .
$$

They form a basis of the space $L^{2}\left(H_{+}^{4}\right)$ in the $S$-coordinate system. This basis corresponds to the chain of subgroups

$$
S O_{0}(1,4) \supset S O(4) \supset S O(3) \supset S O(2) .
$$

\section{The method of orispherical transforms}

Now we consider another problem - expansion of functions $\psi(x) \in L^{2}\left(H_{+}^{4}\right)$ in eigenfunctions of a full collection of self-adjoint operators. Derivation of the inverse formulas will allow us to represent these eigenfunctions in a normed form. Since functions $\psi(x)$ are determined on the hyperboloid $H_{+}^{4}$, then for construction of expansion we can use the method of orispheres, which was worked out by Gel'fand and Graev on the base of integral geometry [24]. This method allows us to go from studying functions $\psi(x)$ on $H_{+}^{4}$ to studying corresponding functions $h(k)$ on the upper sheet $C_{+}^{4}$ of the cone $C^{4}$. Consideration of functions on the cone is convenient, since we can expand them in functions $\Psi(k, \sigma)$ homogeneous in the set of the homogeneous coordinates $k=\left(k_{0}, k_{1}, k_{2}, k_{3}, k_{4}\right)$. Under shifts $\psi(x) \rightarrow \psi\left(g^{-1} x\right), g \in S O_{0}(1,4)$, the functions $\Psi(k, \sigma)$ transform under irreducible representations $\pi^{\sigma}$ of the group $S O_{0}(1,4)$. Let us give an information on the method of orispheres [24, 25].

An orisphere of the hyperboloid $H_{+}^{4}$ is a cut of $H_{+}^{4}$ by the plane

$$
[x, k] \equiv x_{0} k_{0}-x_{1} k_{1}-x_{2} k_{2}-x_{3} k_{3}-x_{4} k_{4}=1,
$$


where $k$ is a fixed point of the cone $C_{+}^{4}$ given by the equation

$$
[k, k] \equiv k_{0} k_{0}-k_{1} k_{1}-k_{2} k_{2}-k_{3} k_{3}-k_{4} k_{4}=0, \quad k_{0}>0 .
$$

Thus, an orisphere on $H_{+}^{4}$ is given by a point $k \in C_{+}^{4}$. Then the set of orispheres can be considered as the set of all points of $C_{+}^{4}$.

According to the method of orispheres, to each finite function $\psi(x)$ on $H_{+}^{4}$ there corresponds a function $h(\omega)$, determined on the set of orispheres $\omega$ of $H_{+}^{4}$. This correspondence is given by the Gel'fand-Graev formula

$$
h(\omega)=\int_{\omega} d \omega \psi(x),
$$

where the integral of the function $\psi(x)$ on the orisphere $\omega:[x, k]=1$ is defined by the formula

$$
\int_{\omega} d \omega \psi(x)=\int_{H_{+}^{4}} \frac{d^{4} x}{x_{0}} \psi(x) \delta([x, k]-1) .
$$

Here $d^{4} x / x_{0}=d x_{1} d x_{2} d x_{3} d x_{4} / x_{0}$ is an invariant (with respect to $S O_{0}(1,4)$ ) measure on $H_{+}^{4}$. Under shifts of points $x$ and $k$ by an element $g \in S O_{0}(1,4)$ the bilinear form $[x, k]$ and the measure $d^{4} x / x_{0}$ are conserved. For this reason, the measure $d \omega$, determined by formula (6.2), is conserved under shifts $\omega \rightarrow g \omega$. This means that

$$
\int_{\omega} d \omega \psi(g x)=\int_{g \omega} d \omega_{g} \psi(x), \quad g \in S O_{0}(1,4),
$$

where $d \omega_{g}$ is a measure on the orisphere $g \omega$. In particular, if a shift $g$ leaves an orisphere $\omega$ invariant, then

$$
\int_{\omega} d \omega \psi(g x)=\int_{\omega} d \omega \psi(x)
$$

Since there exists a one-to-one correspondence between points of the set of orispheres $\omega$ of $H_{+}^{4}$ and points $k \in C_{+}^{4}$, the functions $h(\omega)$ can be considered as functions on $C_{+}^{4}$. Instead of $h(\omega)$ we shall write $h(k)$. Then according to (6.1) and (6.2) we have

$$
h(k)=\int_{H_{+}^{4}} \frac{d^{4} x}{x_{0}} \psi(x) \delta([x, k]-1) .
$$

This transform, turning a function $\psi(x)$ into a function $h(k)$, is called Gel'fand-Graev integral transform.

It is proved in [39] that if $\psi(x)$ is infinitely differentiable finite function on $H_{+}^{4}$, then the function $h(k)$ on $C_{+}^{4}$ is infinitely differentiable, finite, and vanishes inside of some neighborhood of the point $k=0$ of the cone $C_{+}^{4}$.

The inverse formula for the integral transform (6.3) is of the form [39]

$$
\psi(x)=\frac{\Gamma(4)}{(2 \pi)^{4}} \int_{C_{+}^{4}} \frac{d^{4} k}{k_{0}} h(k)([x, k]-1)^{-4},
$$

where $d^{4} k / k_{0}=d k_{1} d k_{2} d k_{3} d k_{4} / k_{0}$ is an invariant (with respect to $S O_{0}(1,4)$ ) measure on the cone $C_{+}^{4}$. Here the integral is understood in the sense of a value, regularized by analytic continuation in a power:

$$
\int_{C_{+}^{4}} \frac{d^{4} k}{k_{0}} h(k)([x, k]-1)^{-4}=\left.\int_{C_{+}^{4}} \frac{d^{4} k}{k_{0}} h(k)([x, k]-1)^{\tau}\right|_{\tau \rightarrow-4} .
$$


We wish to know a form of the operator $\pi(g)$ of the quasi-regular representation (2.11) of $S O_{0}(1,4)$ (restricted onto the space of infinitely differentiable finite functions on $H_{+}^{4}$ ) under the transform (6.3). It is easy to see that this transform turns the function $\pi(g) \psi(x)=\psi\left(g^{-1} x\right)$ on $H_{+}^{4}$ into the function $h_{g}(k) \equiv h\left(g^{-1} k\right)$ on $C_{+}^{4}$. Therefore, to the shift operators $\pi(g)$ on $H_{+}^{4}$ there corresponds shift operators $\hat{\pi}(g)$ on the space of functions $h(k)$ on $C_{+}^{4}$ :

$$
\hat{\pi}(g) h(k)=h\left(g^{-1} k\right), \quad g \in S O_{0}(1,4) .
$$

This equality determines the quasi-regular representation of $S O_{0}(1,4)$ on the space of infinitely differentiable functions $h(k)$ on the cone $C_{+}^{4}$, satisfying an additional symmetry condition [39].

A function $h(k)$ on $C_{+}^{4}$ can be expanded in functions on the cone $C_{+}^{4}$ homogeneous in coordinates $k=\left(k_{0}, k_{1}, k_{2}, k_{3}, k_{4}\right)$ :

$$
\Psi(k, \sigma)=\int_{0}^{\infty} d t h(t k) t^{-\sigma-1}, \quad \sigma \in \mathbb{C} .
$$

These functions have homogeneity degrees $\sigma$ :

$$
\Psi(a k, \sigma)=a^{\sigma} \Psi(k, \sigma), \quad a>0 .
$$

This expansion has the form (see, for example, [25])

$$
h(k)=\frac{1}{2 \pi \mathrm{i}} \int_{\delta-\mathrm{i} \infty}^{\delta+\mathrm{i} \infty} d \sigma t^{\sigma} \Psi(k, \sigma),
$$

where a value $\delta$ is taken in such a way that the function $\Psi(k, \sigma)$ does not have poles on the strip $0 \leqslant \operatorname{Re} \sigma \leqslant \delta$.

It is easy to check that to the function $h_{g}(k)=\hat{\pi}(g) h(k)=h\left(g^{-1} k\right)$ on the cone $C_{+}^{4}$ there corresponds a homogeneous function $\Psi_{g}(k, \sigma)=\Psi\left(g^{-1} k, \sigma\right), \sigma \in \mathbb{C}$, on $C_{+}^{4}$. Thus, to the quasiregular representation $\hat{\pi}$ on the space of functions $h(k)$ on $C_{+}^{4}$ there corresponds a collection of representations $T^{\sigma}, \sigma \in \mathbb{C}$, and $T^{\sigma}$ acts on the space of homogeneous functions $\Psi(k, \sigma)$ on $C_{+}^{4}$ by the formula

$$
T^{\sigma}(g) \Psi(k, \sigma)=\Psi\left(g^{-1} k, \sigma\right), \quad g \in S O_{0}(1,4) .
$$

The representation $T^{\sigma}$ is irreducible for all values of $\sigma$ except for the case when $\sigma$ or $-\sigma-3$ is a non-negative integer. Therefore, the representations $T^{\sigma}$ are irreducible components of the representation $\hat{\pi}$ or of the representation $\pi$, equivalent to $\hat{\pi}$. Note that for $\sigma=\mathrm{i} \rho-3 / 2, \rho \in \mathbb{R}$, the representation $T^{\sigma}$ is unitary.

The representation $T^{\sigma}, \sigma \in \mathbb{C}$, in fact, coincides with the representation $\pi^{\sigma}$ considered in Section 2. To show this, we note that the representation $\pi^{\sigma}$ acts in the space of functions $f(g)$ on $S O_{0}(1,4)$ such that

$$
f(g m n h)=\exp (-\lambda(\log h)) f(g), \quad m \in M=S O(3), \quad n \in N, \quad h \in A=\exp \mathfrak{a},
$$

where $\lambda$ is a linear form on the commutative subalgebra $\mathfrak{a}$ of the Lie algebra so(1,4), determined by the number $\sigma$ (see Section 2). This representation is given by the formula

$$
\pi^{\sigma}\left(g_{0}\right) f(g)=f\left(g_{0}^{-1} g\right) .
$$

The product $m n h, m \in M, n \in N, h \in A$, has the property $m n h=h m^{\prime} n^{\prime}, m^{\prime} \in M, n^{\prime} \in N$. Thus, the functions $f(g)$, on which the representation $\pi^{\sigma}$ is defined, satisfy the condition

$$
f(g h m n)=\exp (-\lambda(\log h)) f(g), \quad m \in M, \quad n \in N, \quad h \in A .
$$


This means that the representation $\pi^{\sigma}$ can be realized on functions $f(x)$ on the space $X=$ $S O_{0}(1,4) / M N$, satisfying the condition $f(x h)=\exp (-\lambda(\log h)) f(x)$. Since $M N=S O(3) \times$ $T(3)$, then $X=C_{+}^{4}$. Besides, the latter condition is the condition of homogeneity (of degree $\sigma$ ) of the function $f(x)$ with respect to the collection of variables $k_{0}, k_{1}, k_{2}, k_{3}, k_{4}$. This shows that the representations $T^{\sigma}$ and $\pi^{\sigma}$ are equivalent.

Using the formulas (6.4)-(6.6) we can find a connection between the functions $\psi(x)$ and $\Psi(k, \sigma)[9]$ :

$$
\begin{aligned}
& \psi(x)=\frac{\mathrm{i}}{2(2 \pi)^{4}} \int_{\delta-i \infty}^{\delta+i \infty} \frac{\Gamma(\sigma+3)}{\Gamma(\sigma)} \cot \pi \sigma \int_{\Gamma} d \mu\left(k^{\prime}\right) \Psi\left(k^{\prime}, \sigma\right)\left[x, k^{\prime}\right]^{-\sigma-3}, \\
& \Psi\left(k^{\prime}, \sigma\right)=\int_{H_{+}^{4}} \frac{d^{4} x}{x_{0}} \psi(x)\left[x, k^{\prime}\right]^{\sigma},
\end{aligned}
$$

where $-3<\delta<1$ (this condition for $\delta$ is connected with poles of the function $\Gamma(\sigma+3) \cot \pi \sigma / \Gamma(\sigma)$ ). Integration contour $\Gamma$ in the formula (6.7) is any surface on the cone $C_{+}^{4}$ intersecting once each generator of the cone, and $d \mu\left(k^{\prime}\right)$ is an invariant measure on $\Gamma$ determined by means of the invariant measure $d^{4} k / k_{0}$ on the cone $C_{+}^{4}$ using the equality

$$
d^{4} k / k_{0}=t^{2} d t d \mu\left(k^{\prime}\right)
$$

where $k \in C_{+}^{4}$ and $k^{\prime} \in \Gamma$ are connected by $k=t k^{\prime}$.

Below we shall consider cuts of the cone by the surfaces $k_{4}= \pm 1, k_{0}-k_{4}=1, k_{0}^{2}-k_{4}^{2}=1$, $k_{0}^{2}-k_{1}^{2}-k_{2}^{2}=1, k_{0}=1$. To these choices of $\Gamma$ there will correspond expansions of the function $\psi(x)$ in basis functions in the respective coordinate systems on $H_{+}^{4}$. In order to obtain an expansion of $\psi(x)$ in basis functions on $H_{+}^{4}$, we use formulas (6.7) and (6.8). First we expand the functions $\Psi\left(k^{\prime}, \sigma\right)$ in basis functions on $\Gamma$ and then take the corresponding integrals over $\Gamma$. Let us consider expansions of $\psi(x)$ for each of the cuts, given above.

\section{Expansion of functions on the hyperboloid}

\subsection{Expansion in $\boldsymbol{H}$-coordinate system}

The cone $C_{+}^{4}$ is determined by the relations

$$
C_{+}^{4}:[k, k]=k_{0}^{2}-k_{1}^{2}-k_{2}^{2}-k_{3}^{2}-k_{4}^{2}=0, \quad k_{0}>0 .
$$

We consider the cut of $C_{+}^{4}$ by the surfaces $k_{4}= \pm 1$. We obtain the contour $\Gamma$ coinciding with upper sheets of two hyperboloids

$$
H_{\varepsilon}^{3}: k_{0}^{2}-k_{1}^{2}-k_{2}^{2}-k_{3}^{2}=1, \quad k_{4}=\varepsilon= \pm 1 .
$$

The motion group of the hyperboloid $k_{0}^{2}-k_{1}^{2}-k_{2}^{2}-k_{3}^{2}=1$ is isomorphic to the Lorentz group $S O_{0}(1,3)$ (see [25]). We denote points of this hyperboloid by $k^{\prime}: k_{0}^{\prime 2}-k_{1}^{\prime 2}-k_{2}^{\prime 2}-k_{3}^{\prime 2}=1$. If $k_{4}=+1$ we have the hyperboloid $H_{+}^{3}$, and if $k_{4}=-1$ then we have the hyperboloid $H_{-}^{3}$. Thus, the contour $\Gamma$ consists of two parts: $\Gamma=\Gamma_{+} \cup \Gamma_{-}$.

Expansion of functions given on the 3-dimensional hyperboloid in different coordinate systems is derived in [9]. In the spherical coordinate system, corresponding to the reduction $S O_{0}(1,3) \supset$ $S O(3) \supset S O(2)$, the expansion is of the form

$$
\phi\left(k^{\prime}\right)=\frac{\mathrm{i}}{2} \sum_{l=0}^{\infty} \sum_{m=-l}^{l} \int_{\gamma-\mathrm{i} \infty}^{\gamma+i \infty} d \tau C_{l m}(\tau) \frac{\Gamma(-\tau+1)}{\Gamma(-\tau-l-1)}(\sinh c)^{-1 / 2} P_{\tau+1 / 2}^{-l-1 / 2}(\cosh c) Y_{l m}(\Theta, \Phi),
$$




$$
C_{l m}(\tau)=\frac{\Gamma(\tau+1)}{\Gamma(\tau-l+1)} \int_{H_{+}^{3}} d k^{\prime} \phi\left(k^{\prime}\right)(\sinh c)^{-1 / 2} P_{-\tau-3 / 2}^{-l-1 / 2}(\cosh c) \overline{Y_{l m}(\Theta, \Phi)}
$$

where $Y_{l m}(\theta, \Phi)$ is the $S O(3)$-spherical function

$$
Y_{l m}(\Theta, \Phi)=\left[\frac{2 l+1}{4 \pi} \frac{(l-m) !}{(l+m) !}\right]^{1 / 2} P_{l}^{m}(\cos \Theta) e^{\mathrm{i} m \Phi}
$$

and

$$
\begin{array}{ll}
k_{0}^{\prime}=\cosh c, & k_{1}^{\prime}=\sinh c \sin \Theta \cos \Phi, \quad k_{2}^{\prime}=\sinh c \sin \Theta \sin \Phi, \quad k_{3}^{\prime}=\sinh c \cos \Theta, \\
0 \leqslant c<\infty, & 0 \leqslant \Theta<\pi, \quad 0 \leq \Phi<2 \pi, \quad d k^{\prime} \equiv \sinh ^{2} c \sin \Theta d c d \Theta d \Phi .
\end{array}
$$

Applying this decomposition to the functions $\Psi_{+}\left(k^{\prime}, \sigma\right)$ (with $k^{\prime} \in \Gamma_{+} \equiv H_{+}^{3}$ ) and $\Psi_{-}\left(k^{\prime}, \sigma\right)$ (with $k^{\prime} \in \Gamma_{-} \equiv H_{-}^{3}$ ), substituting this expressions into (6.7) and permuting an order of integrals, we obtain

$$
\begin{aligned}
\psi(x)= & -\frac{1}{4(2 \pi)^{4}} \sum_{l, m, \varepsilon} \int_{\delta-\mathrm{i} \infty}^{\delta+\mathrm{i} \infty} d \sigma(\sigma+2)(\sigma+1) \sigma \cot \pi \sigma \int_{\gamma-\mathrm{i} \infty}^{\gamma+\mathrm{i} \infty} d \tau \frac{\Gamma(-\tau+2)}{\Gamma(-\tau-l-1)} C_{l m}^{\varepsilon}(\tau, \sigma) \\
& \times \int_{\Gamma_{\varepsilon}} d k^{\prime}\left[x, k^{\prime}\right]^{-\sigma-3}(\sinh c)^{-1 / 2} P_{\tau+1 / 2}^{-l-1 / 2}(\cosh c) Y_{l m}(\Theta, \Phi) .
\end{aligned}
$$

Note that the point $x$ belongs to $H_{+}^{4}$, and $c, \Theta, \Phi$ parametrize points of the hyperboloids $H_{\varepsilon}^{3}$.

Let us calculate the integrals over the contours $\Gamma_{\varepsilon}$ in (7.2):

$$
J_{\sigma \tau l m}^{\varepsilon}(x)=\int_{\Gamma_{\varepsilon}} d k^{\prime}\left[x, k^{\prime}\right]^{-\sigma-3}(\sinh c)^{-1 / 2} P_{\tau+1 / 2}^{-l-1 / 2}(\cosh c) P_{l}^{m}(\cos \Theta) e^{\mathrm{i} m \Phi} .
$$

First we calculate the integrals for the point $x^{0}=(\cosh a, 0,0,0, \sinh a)$ corresponding to the hyperbolic rotation $g_{04}(a) \in S O_{0}(1,4)$, and then, by means of transformations of the subgroup $S O_{0}(1,3)$, we go from $x^{0}$ to $x$. For $x^{0}$ we have

$$
J_{\sigma \tau}^{\varepsilon}(a)=4 \pi \int_{1}^{\infty} d(\cosh c)(\cosh a \cosh c-\varepsilon \sinh a)^{-\sigma-3} \sinh ^{1 / 2} c \times P_{\tau+1 / 2}^{-1 / 2}(\cosh c),
$$

where $J_{\sigma \tau}^{\varepsilon}(a) \equiv J_{\sigma \tau 00}^{\varepsilon}\left(x^{0}\right)$. We calculate the integral

$$
J_{\sigma \tau}(u)=\int_{1}^{\infty} d y\left(y^{2}-1\right)^{1 / 4} P_{\tau+1 / 2}^{-1 / 2}(y)(y+u)^{-\sigma-3}, \quad u \equiv-\varepsilon \tanh a .
$$

For this we substitute into (7.4) the expression

$$
P_{\tau+1 / 2}^{-1 / 2}(y)=\frac{\left(y^{2}-1\right)^{-1 / 4}}{\sqrt{\pi}} \int_{1}^{y} d t(y-t)^{-1 / 2} P_{\tau+1 / 2}(t)
$$

(see formula $3.7(29)$ in [40]) for $P_{\tau+1 / 2}^{-1 / 2}(y)$ :

$$
J_{\sigma \tau}(u)=\frac{1}{\sqrt{\pi}} \int_{1}^{\infty} d y \int_{1}^{y} d t(y+u)^{-\sigma-3}(y-t)^{-1 / 2} P_{\tau+1 / 2}(t) .
$$

The following integral representation holds for the function $P_{\tau+1 / 2}(t)$ :

$$
P_{\tau+1 / 2}(t)=\frac{2^{-\tau-1 / 2}}{2 \pi \mathrm{i}} \int_{C} d t^{\prime} \frac{\left(t^{\prime 2}-1\right)^{\tau+1 / 2}}{\left(t^{\prime}-t\right)^{\tau+3 / 2}},
$$


where the contour $C$ is a closed curve in the plane of complex variable $t^{\prime}$, containing inside the points 1 and $t$; integration is in the counter-clockwise direction. Therefore,

$$
J_{\sigma \tau}(u)=\frac{2^{-\tau-3 / 2}}{i \pi^{3 / 2}} \int_{1}^{\infty} d y(y+u)^{-\sigma-3} \int_{1}^{y} d t(y-t)^{-1 / 2} \int_{C} d t^{\prime}\left(t^{\prime 2}-1\right)^{\tau+1 / 2}\left(t^{\prime}-t\right)^{-\tau-3 / 2} .
$$

Making some transformations (see [17]) we reduce this expression for $J_{\sigma \tau}(u)$ to the form

$$
\begin{aligned}
J_{\sigma \tau}(u) & =\lambda \int_{1}^{\infty} d y \int_{1}^{y} d t^{\prime} \frac{\left(t^{\prime 2}-1\right)^{\tau+1 / 2}}{\left(y-t^{\prime}\right)^{\tau+1}(y+u)^{\sigma+3}} \\
& =\lambda \int_{1}^{\infty} d t^{\prime}\left(t^{\prime 2}-1\right)^{\tau+1 / 2} \int_{t^{\prime}}^{\infty} d y\left(y-t^{\prime}\right)^{-\tau-1}(y+u)^{-\sigma-3},
\end{aligned}
$$

where $\lambda=2^{-\tau-1 / 2} \cos \pi \tau \Gamma(-\tau-1 / 2) / \pi \Gamma(-\tau)$. Using the formula 3.196(2) from [40], we get

$$
J_{\sigma \tau}(u)=\lambda \frac{\Gamma(-\tau) \Gamma(\sigma+\tau+3)}{\Gamma(\sigma+3)} \int_{1}^{\infty} d t^{\prime} \frac{\left(t^{\prime 2}-1\right)^{\tau+1 / 2}}{\left(t^{\prime}+u\right)^{\sigma+\tau+3}} .
$$

Since

$$
2^{-\tau-1 / 2} \int_{1}^{\infty} d t^{\prime} \frac{\left(t^{\prime} 2-1\right)^{\tau+1 / 2}}{\left(t^{\prime}+u\right)^{\sigma+\tau+3}}=\frac{\Gamma(\sigma-\tau+1)}{[\Gamma(\tau+3 / 2)]^{-1}}\left(1-u^{2}\right)^{-(\sigma+3 / 2) / 2} P_{\tau+1 / 2}^{-\sigma-3 / 2}(u),
$$

then

$$
J_{\sigma \tau}(u)=-\frac{\Gamma(\sigma+\tau+3) \Gamma(\sigma-\tau+1)}{\Gamma(\sigma+3)}\left(1-u^{2}\right)^{-(\sigma+3 / 2) / 2} P_{\tau+1 / 2}^{-\sigma-3 / 2}(u),
$$

where the equality $\Gamma(-\tau+1 / 2) \Gamma(\tau+1 / 2)=\pi \cos ^{-1}(\pi \tau)$ was used. Thus, we have

$$
J_{\sigma \tau}^{\varepsilon}(a)=-4 \pi \frac{\Gamma(\sigma+\tau+3) \Gamma(\sigma-\tau+1)}{\Gamma(\sigma+3)}(\cosh a)^{-3 / 2} P_{\tau+1 / 2}^{-\sigma-3 / 2}(-\varepsilon \tanh a) .
$$

For any $x=x(a, b, \theta, \varphi)$, where $a, b, \theta, \varphi$ are the parameters from (4.2), the integral $J_{\sigma \tau l m}^{\varepsilon}(x)$ is easily calculated as in [9] and we have

$$
\begin{aligned}
J_{\sigma \tau l m}^{\varepsilon}(x)= & -(2 \pi)^{3 / 2} \frac{\Gamma(\sigma+\tau+3) \Gamma(\sigma-\tau+1)}{\Gamma(\sigma+3)}(\cosh a)^{-3 / 2}(\sinh b)^{-1 / 2} \\
& \times P_{\tau+1 / 2}^{-\sigma-3 / 2}(-\varepsilon \tanh a) P_{\tau+1 / 2}^{-l-1 / 2}(\cosh b) P_{l}^{m}(\cos \theta) e^{\mathrm{i} m \varphi} .
\end{aligned}
$$

Thus, according to the formula (7.2) we get

$$
\begin{aligned}
\psi(x)= & \frac{1}{4(2 \pi)^{5 / 2}} \sum_{l=0}^{\infty} \sum_{m=-l}^{l} \int_{\delta-\mathrm{i} \infty}^{\delta+\mathrm{i} \infty} d \sigma \frac{\cot \pi \sigma}{\Gamma(\sigma)} \\
& \times \int_{\gamma-\mathrm{i} \infty}^{\gamma+\mathrm{i} \infty} d \tau(\tau+1) \tau \Gamma(\sigma-\tau+1) \Gamma(\sigma+\tau+3) V_{\tau l m}(b, \theta, \varphi) \\
& \times\left[C_{l m}^{+}(\tau, \sigma) P_{\tau+1 / 2}^{-\sigma-3 / 2}(-\tanh a)+C_{l m}^{-}(\tau, \sigma) P_{\tau+1 / 2}^{-\sigma-3 / 2}(\tanh a)\right](\cosh a)^{-3 / 2}
\end{aligned}
$$

where

$$
V_{\tau l m}(b, \theta, \varphi)=\frac{\Gamma(-\tau-1)}{\Gamma(-\tau-l-1)}(\sinh b)^{-1 / 2} P_{\tau+1 / 2}^{-l-1 / 2}(\cosh b) Y_{l m}(\theta, \varphi) .
$$

The functions (7.7) coincide in a form with the functions from (7.1). 
The formula (7.6) expresses the functions $\psi(x)$ in terms of the functions $C_{l m}^{ \pm}(\tau, \sigma)$. Let us find an expression for $C_{l m}^{ \pm}(\tau, \sigma)$ in terms of $\psi(x)$. Using the expansions (6.8) and (7.1) we have

$$
\begin{aligned}
C_{l m}^{\varepsilon}(\tau, \sigma)= & \frac{\Gamma(\tau+1)}{\Gamma(\tau-l+1)} \int_{H_{+}^{4}} \frac{d^{4} x}{x_{0}} \psi(x) \\
& \times \int_{\Gamma_{\varepsilon}} d k^{\prime}\left[x, k^{\prime}\right]^{\sigma}(\sinh c)^{-1 / 2} P_{-\tau-3 / 2}^{-l-1 / 2}(\cosh c) \overline{Y_{l m}(\Theta, \Phi)} .
\end{aligned}
$$

Integration over $k^{\prime}$ is fulfilled in the same way as in the case of the integral (7.3). We obtain

$$
\begin{aligned}
C_{l m}^{\varepsilon}(\tau, \sigma)= & (2 \pi)^{3 / 2} \frac{\Gamma(\tau+1) \Gamma(-\sigma-\tau-2) \Gamma(\tau-\sigma)}{\Gamma(\tau-l+1) \Gamma(-\sigma)} \int_{H_{+}^{4}} \frac{d^{4} x}{x_{0}} \psi(x)(\cosh a)^{-3 / 2}(\sinh b)^{-1 / 2} \\
& \times P_{-\tau-3 / 2}^{\sigma+3 / 2}(-\varepsilon \tanh a) P_{-\tau-3 / 2}^{-l-1 / 2}(\cosh b) \overline{Y_{l m}(\theta, \phi)}
\end{aligned}
$$

where the measure $d^{4} x / x_{0}$ in the coordinates $a, b, \theta, \varphi$ has the form

$$
d^{4} x / x_{0}=\cosh ^{3} a \sinh ^{2} b \sin \theta d a d b d \theta d \varphi .
$$

For the unitary case when $\sigma=\mathrm{i} \rho-3 / 2$ (that is, $\delta=-3 / 2)$ and $\tau=\mathrm{i} \nu-1$ (that is $\gamma=-1$ ), where $\rho$ and $\nu$ are real numbers, we receive

$$
\begin{aligned}
& \psi(x)= \frac{1}{(2 \pi)^{5 / 2}} \sum_{l=0}^{\infty} \sum_{m=-l}^{l} \int_{0}^{\infty} d \rho\left(\rho^{2}+1 / 4\right) \rho \tanh \pi \rho \int_{0}^{\infty} d \nu \nu^{2} \\
& \times \frac{\Gamma(\mathrm{i} \rho+\mathrm{i} \nu+1 / 2) \Gamma(\mathrm{i} \rho-\mathrm{i} \nu+1 / 2)}{\Gamma(\mathrm{i} \rho+3 / 2)} V_{\nu l m}(b, \theta, \varphi)(\cosh a)^{-3 / 2} \\
& \times\left[C_{l m}^{+}(\nu, \rho) P_{\mathrm{i} \nu-1 / 2}^{-\mathrm{i} \rho}(-\tanh a)+C_{l m}^{-}(\nu, \rho) P_{\mathrm{i} \nu-1 / 2}^{-\mathrm{i} \rho}(\tanh a)\right] \\
& C_{l m}^{\varepsilon}(\nu, \rho)=(2 \pi)^{3 / 2} \frac{\Gamma(-\mathrm{i} \rho-\mathrm{i} \nu+1 / 2) \Gamma(-\mathrm{i} \rho+\mathrm{i} \nu+1 / 2)}{\Gamma(-\mathrm{i} \rho+3 / 2)} \\
& \quad \times \int_{H_{+}^{4}} \frac{d^{4} x}{x_{0}} \psi(x)(\cosh a)^{-3 / 2} P_{-\mathrm{i} \nu-1 / 2}^{\mathrm{i} \rho}(-\varepsilon \tanh a) \overline{V_{\nu l m}(b, \theta, \phi)},
\end{aligned}
$$

where

$$
V_{\nu l m}(b, \theta, \varphi)=\frac{\Gamma(\mathrm{i} \nu)}{\Gamma(\mathrm{i} \nu-l)}(\sinh b)^{-1 / 2} P_{\mathrm{i} \nu-1 / 2}^{-l-1 / 2}(\cosh b) Y_{l m}(\theta, \phi) .
$$

Here we have taken into account that

$$
\begin{aligned}
& \tau(\tau+1) d \tau=-\nu(\mathrm{i} \nu-1) d \nu, \quad \cot \pi \sigma=-\mathrm{i} \tanh \pi \rho \\
& (\sigma+2)(\sigma+1) \sigma d \sigma=-\mathrm{i}\left(\rho^{2}+1 / 4\right)(\mathrm{i} \rho-3 / 2) d \rho \\
& \int_{-\infty}^{\infty} d \rho \Omega(\rho)(\cdots)=\int_{-\infty}^{0} d \rho \Omega(\rho)(\cdots)+\int_{0}^{\infty} d \rho \Omega(\rho)(\cdots) \\
& =2 \mathrm{i} \int_{0}^{\infty} d \rho \rho\left(\rho^{2}+1 / 4\right) \tanh \pi \rho(\cdots),
\end{aligned}
$$

where $\Omega(\rho)=\left(\rho^{2}+1 / 4\right)(\mathrm{i} \rho-3 / 2) \tanh \pi \rho$ and $(\cdots)$ is the expression under the sign of the integral over $\rho$ in (7.9).

The formula (7.9) is an expansion of the function $\psi(x)$ in basis functions (5.12). It follows from the expansions (7.9)-(7.11) that the functions (5.12) have the following normed form:

$$
\Phi_{\rho \nu l m}^{\varepsilon}(a, b, \theta, \varphi)=\frac{|\Gamma(\mathrm{i} \rho+\mathrm{i} \nu+1 / 2) \Gamma(\mathrm{i} \rho-\mathrm{i} \nu+1 / 2) \Gamma(\mathrm{i} \nu)|}{\sqrt{2 \pi}|\Gamma(\mathrm{i} \rho+3 / 2) \Gamma(\mathrm{i} \nu-l)|}(\cosh a)^{-3 / 2}
$$




$$
\times P_{\mathrm{i} \nu-1 / 2}^{-\mathrm{i} \rho}(\varepsilon \tanh a)(\sinh b)^{-1 / 2} P_{\mathrm{i} \nu-1 / 2}^{-l-1 / 2}(\cosh b) Y_{l m}(\theta, \phi) .
$$

This functions are normed by the formula

$$
\begin{aligned}
\left\langle\Phi_{\rho^{\prime} \nu^{\prime} l^{\prime} m^{\prime}}^{\varepsilon^{\prime}}, \Phi_{\rho \nu l m}^{\varepsilon}\right\rangle & =\int_{H_{+}^{4}} \frac{d^{4} x}{x_{0}} \Phi_{\rho \nu l m}^{\varepsilon}(a, b, \theta, \varphi) \overline{\Phi_{\rho^{\prime} \nu^{\prime} l^{\prime} m^{\prime}}^{\varepsilon^{\prime}}(a, b, \theta, \varphi)} \\
& =\frac{\delta\left(\rho-\rho^{\prime}\right)}{\rho\left(\rho^{2}+1 / 4\right) \tanh \pi \rho} \frac{\delta\left(\nu-\nu^{\prime}\right)}{\nu^{2}} \delta_{l l^{\prime}} \delta_{m m^{\prime}} \delta_{\varepsilon \varepsilon^{\prime}} .
\end{aligned}
$$

The Plancherel formula for the transforms (7.9)-(7.10) is of the form

$$
\begin{aligned}
\int_{H_{+}^{4}} \frac{d^{4} x}{x_{0}}|\psi(x)|^{2}= & \frac{1}{(2 \pi)^{4}} \sum_{l=0}^{\infty} \sum_{m=-l}^{l} \int_{0}^{\infty} d \rho\left(\rho^{2}+1 / 4\right) \rho \tanh \pi \rho \\
& \times \int_{0}^{\infty} d \nu \nu^{2}\left[\left|C_{l m}^{+}(\nu, \rho)\right|^{2}+\left|C_{l m}^{-}(\nu, \rho)\right|^{2}\right] .
\end{aligned}
$$

\subsection{Expansion in $\boldsymbol{O}$-coordinate system}

In this case, the cut of $C_{+}^{4}$ by the plane $k_{0}-k_{4}=1$ is considered as a contour $\Gamma$. We parametrize this contour $\Gamma \equiv \Gamma_{O}$ by the coordinates $\zeta, \Theta, \Phi$ :

$$
\begin{array}{ll}
k_{0}^{\prime}=\left(1+\zeta^{2}\right) / 2, & k_{1}^{\prime}=\zeta \sin \Theta \cos \Phi, \quad k_{2}^{\prime}=\zeta \sin \Theta \sin \Phi, \\
k_{3}^{\prime}=\zeta \cos \Theta, \quad k_{4}^{\prime}=\left(-1+\zeta^{2}\right) / 2, \\
0 \leqslant \zeta<\infty, \quad 0 \leqslant \Theta<\pi, \quad 0 \leqslant \Phi<2 \pi .
\end{array}
$$

We expand the function $\Psi\left(k^{\prime}, \sigma\right)$, considered on this contour $\Gamma_{0}$, in the spherical Bessel functions

$$
j_{l}(\kappa \zeta)=\left(\frac{\pi}{2 \kappa \zeta}\right)^{1 / 2} J_{l+1 / 2}(\kappa \zeta)
$$

and spherical harmonics $Y_{l m}(\Theta, \Phi)$. Then

$$
\Psi\left(k^{\prime}, \sigma\right)=\sum_{l=0}^{\infty} \sum_{m=-l}^{l} \int_{0}^{\infty} d \kappa \kappa^{2} A_{l m}(\kappa, \sigma) \Psi_{\kappa l m}\left(k^{\prime}\right),
$$

where $\Psi_{\kappa l m}\left(k^{\prime}\right)=j_{l}(\kappa \zeta) Y_{l m}(\Theta, \Phi)$. The inverse transform is of the form

$$
A_{l m}(\kappa, \sigma)=\frac{2}{\pi} \int_{\Gamma_{O}} d k^{\prime} \Psi\left(k^{\prime}, \sigma\right) \overline{\Psi_{\kappa l m}\left(k^{\prime}\right)}
$$

where $d k^{\prime}=\zeta^{2} \sin \Theta d \zeta d \Theta d \Phi$. Now we have

$$
\begin{aligned}
\psi(x)= & \frac{\mathrm{i}}{2(2 \pi)^{4}} \sum_{l=0}^{\infty} \sum_{m=-l}^{l} \int_{\delta-\mathrm{i} \infty}^{\delta+\mathrm{i} \infty} d \sigma \frac{\Gamma(\sigma+3)}{\Gamma(\sigma)} \cot \pi \sigma \int_{0}^{\infty} d \kappa \kappa^{2} A_{l m}(\kappa, \sigma) \\
& \times \int_{\Gamma_{O}} d k^{\prime}\left[x, k^{\prime}\right]^{-\sigma-3} \Psi_{\kappa l m}\left(k^{\prime}\right) .
\end{aligned}
$$

We have to calculate the integral

$$
J_{l m}^{\kappa \sigma}\left(x \equiv=\int_{\Gamma_{O}} d k^{\prime}\left[x, k^{\prime}\right]^{-\sigma-3}\left(\frac{\pi}{2 \kappa \zeta}\right)^{1 / 2} J_{l+1 / 2}(\kappa \zeta) Y_{l m}(\Theta, \Phi) .\right.
$$


Due to orthogonality of the Legendre functions $P_{l}^{m}(\cos \Theta)$ and orthogonality of the exponential functions $\exp \operatorname{im} \Phi$, for the point $x^{0}=x^{0}(a)=(\cosh a, 0,0,0,-\sinh a)$ we have $J_{l m}^{\kappa \sigma}\left(x^{0}\right)=0$ for $l \neq m, m \neq 0$, and

$$
J_{00}^{\kappa \sigma}\left(x_{0}\right)=\pi\left(2 e^{-a}\right)^{\sigma+3} \int_{0}^{\infty} d \zeta \zeta^{2}\left(e^{-2 a}+\zeta\right)^{-\sigma-3}\left(\frac{2}{\kappa \zeta}\right)^{1 / 2} J_{1 / 2}(\kappa \zeta) .
$$

Setting $e^{-a}=b$ and using the formula 6.565(4) in [40], we get

$$
J_{00}^{\kappa \sigma}\left(x^{0}\right)=(2 b)^{3 / 2} \pi \kappa^{\sigma+3 / 2} \frac{1}{\Gamma(\sigma+3)} K_{\sigma+3 / 2}(\kappa b) .
$$

For arbitrary $x$ we have (see [9])

$$
J_{l m}^{\kappa \sigma}(x)=\sqrt{4 \pi} J_{00}^{\kappa \sigma}\left(x^{0}(a)\right) \Psi_{\kappa l m}(r, \theta, \varphi) .
$$

Thus,

$$
\begin{aligned}
\psi(x)= & \frac{\mathrm{i} b^{3 / 2}}{(2 \pi)^{5 / 2}} \sum_{l=0}^{\infty} \sum_{m=-l}^{l} \int_{\delta-\mathrm{i} \infty}^{\delta+\mathrm{i} \infty} d \sigma \frac{\cot \pi \sigma}{\Gamma(\sigma)} \int_{0}^{\infty} d \kappa A_{l m}(\kappa, \sigma) \\
& \times \kappa^{\sigma+7 / 2} K_{\sigma+3 / 2}(\kappa b) \Psi_{\kappa l m}(r, \theta, \varphi), \quad b=e^{-a},
\end{aligned}
$$

where $\Psi_{\kappa l m}$ is such function as in (7.16) but determined for other variables.

In order to express $A_{l m}(\kappa, \sigma)$ in terms of $\Psi\left(k^{\prime}, \sigma\right)$, we substitute the expression (6.8) into (7.17):

$$
A_{l m}(\kappa, \sigma)=\frac{2}{\pi} \int_{H_{+}^{4}} \frac{d^{4} x}{x_{0}} \psi(x) \int_{\Gamma_{0}} d k^{\prime}\left[x, k^{\prime}\right]^{\sigma} \overline{\Psi_{\kappa l m}\left(k^{\prime}\right)} .
$$

Integrating over $k^{\prime}$ we obtain

$$
A_{l m}(\kappa, \sigma)=\frac{8(2 \pi)^{1 / 2} \kappa^{-\sigma-3 / 2}}{\Gamma(-\sigma)} \int_{H_{+}^{4}} \frac{d^{4} x}{x_{0}} \psi(x) b^{3 / 2} K_{\sigma+3 / 2}(\kappa b) \overline{\Psi_{\kappa l m}(r, \theta, \varphi)},
$$

where

$$
d^{4} x / x_{0}=e^{3 a} r^{2} \sin \theta d a d r d \theta d \varphi .
$$

For the unitary case, when $\sigma=\mathrm{i} \rho-3 / 2, \rho \in \mathbb{R}, \delta=-3 / 2$, the relations (7.19) and (7.20) take the form

$$
\begin{gathered}
\psi(x)=\frac{b^{3 / 2}}{\pi(2 \pi)^{3 / 2}} \sum_{l=0}^{\infty} \sum_{m=-l}^{l} \int_{0}^{\infty} d \rho\left(\rho^{2}+1 / 4\right) \rho \frac{\tanh \pi \rho}{\Gamma(\mathrm{i} \rho+3 / 2)} \\
\times \int_{0}^{\infty} d \kappa \kappa^{2} A_{l m}(\kappa, \rho) \kappa^{\mathrm{i} \rho} K_{\mathrm{i} \rho}(\kappa b) \Psi_{\kappa l m}(r, \theta, \varphi), \\
A_{l m}(\kappa, \rho)=\frac{8(2 \pi)^{1 / 2} \kappa^{-\mathrm{i} \rho}}{\Gamma(-\mathrm{i} \rho+3 / 2)} \int_{H_{+}^{4}} \frac{d^{4} x}{x_{0}} \psi(x) b^{3 / 2} K_{\mathrm{i} \rho}(\kappa b) \overline{\Psi_{\kappa l m}(r, \theta, \varphi)} .
\end{gathered}
$$

The formula (7.21) is an expansion of the function $\psi(x)$ in basis elements (5.22). The formulas (7.21) and (7.22) show that the functions (5.22) in a normed form have the form

$$
\Phi_{l m}^{\rho \kappa}(b, r, \theta, \varphi)=\frac{(2 / \pi \kappa r)^{1 / 2} b^{3 / 2}}{|\Gamma(i \rho+3 / 2)|} K_{\mathrm{i} \rho}(\kappa b) J_{l+1 / 2}(\kappa r) Y_{l m}(\theta, \varphi) .
$$


The normalization condition is

$$
\left\langle\Phi_{l^{\prime} m^{\prime}}^{\rho^{\prime} \kappa^{\prime}}, \Phi_{l m}^{\rho \kappa}\right\rangle=\frac{\delta\left(\rho-\rho^{\prime}\right)}{\rho\left(\rho^{2}+1 / 4\right) \tanh \pi \rho} \frac{\delta\left(\kappa-\kappa^{\prime}\right)}{\kappa^{2}} \delta_{l l^{\prime}} \delta_{m m^{\prime}} .
$$

The Plancherel formula for the transforms $(7.21)-(7.22)$ is

$$
\int_{H_{+}^{4}} \frac{d^{4} x}{x_{0}}|\psi(x)|^{2}=\frac{1}{4(2 \pi)^{3}} \sum_{l=0}^{\infty} \sum_{m=-l}^{l} \int_{0}^{\infty} d \rho\left(\rho^{2}+\frac{1}{4}\right) \rho \tanh \pi \rho \int_{0}^{\infty} d \kappa \kappa^{2}\left|A_{l m}(\kappa, \rho)\right|^{2} .
$$

\subsection{Expansion in $O C$-coordinate system}

We leave in this case the contour $\Gamma_{O}$ of the previous subsection and introduce on it the coordinates $\zeta, s, \Phi$ :

$$
\begin{array}{llll}
k_{0}^{\prime}=\left(\zeta^{2}+s^{2}+1\right) / 2, & k_{1}^{\prime}=\zeta \cos \Phi, & k_{2}^{\prime}=\zeta \sin \Phi, & k_{3}^{\prime}=s, \\
k_{4}^{\prime}=\left(\zeta^{2}+s^{2}-1\right) / 2, & 0 \leqslant \zeta<\infty, & -\infty<s<\infty, & 0 \leqslant \Phi<2 \pi .
\end{array}
$$

We expand the function $\Psi\left(k^{\prime}, \sigma\right)$ in basis functions on $\Gamma_{O}$,

$$
\Psi_{\eta q}^{m}\left(k^{\prime}\right) \equiv \Psi_{\eta q}^{m}(\zeta, s, \Phi)=\frac{1}{2 \pi} J_{m}(\eta \zeta) e^{\mathrm{i} q s} e^{\mathrm{i} m \Phi} .
$$

This expansion is of the form

$$
\begin{aligned}
& \Psi\left(k^{\prime}, \sigma\right)=\sum_{m=-\infty}^{\infty} \int_{0}^{\infty} d \eta \eta \int_{-\infty}^{\infty} d q A_{m}(\eta, q, \sigma) \Psi_{\eta q}^{m}\left(k^{\prime}\right), \\
& A_{m}(\eta, q, \sigma)=\int_{\Gamma_{O}} d k^{\prime} \Psi\left(k^{\prime}, \sigma\right) \overline{\Psi_{\eta q}^{m}\left(k^{\prime}\right)}
\end{aligned}
$$

where $d k^{\prime}=\zeta d \zeta d s d \Phi$. Substituting the expression (7.28) into (6.7), we get

$$
\begin{aligned}
\psi(x)= & \frac{\mathrm{i}}{2(2 \pi)^{4}} \sum_{m=-\infty}^{\infty} \int_{\delta-\mathrm{i} \infty}^{\delta+\mathrm{i} \infty} d \sigma \frac{\Gamma(\sigma+3)}{\Gamma(\sigma)} \cot \pi \sigma \int_{0}^{\infty} d \eta \eta \int_{-\infty}^{\infty} d q A_{m}(\eta, q, \sigma) \\
& \times \int_{\Gamma_{O}} d k^{\prime}\left[x, k^{\prime}\right]^{-\sigma-3} \Psi_{\eta q}^{m}\left(k^{\prime}\right) .
\end{aligned}
$$

The integral

$$
J_{m}^{\eta q \sigma}(x)=\int_{\Gamma_{O}} d k^{\prime}\left[x, k^{\prime}\right]^{-\sigma-3} \Psi_{\eta q}^{m}\left(k^{\prime}\right)
$$

for $x=x^{0}(a)=(\cosh a, 0,0,0,-\sinh a)$ and $m=0$ takes the form

$$
J_{0}^{\eta q \sigma}\left(x^{0}(a)\right)=(2 b)^{\sigma+3} \int_{0}^{\infty} d \zeta \zeta J_{0}(\eta \zeta) \int_{-\infty}^{\infty} d s e^{\mathrm{i} q s}\left(\zeta^{2}+s^{2}+b^{2}\right)^{-\sigma-3},
$$

where $e^{-a} \equiv b$. Using the formula

$$
\int_{-\infty}^{\infty} d y\left(1+y^{2}\right)^{\lambda} e^{\mathrm{i} y t}=\frac{2 \sqrt{\pi}}{\Gamma(-\lambda)}\left(\frac{|t|}{2}\right)^{-\lambda-1 / 2} K_{-\lambda-1 / 2}(|t|),
$$

by means of the relation $6.596(7)$ in [40] we get

$$
J_{0}^{\eta q \sigma}(a)=2(2 \pi)^{1 / 2} b^{3 / 2} \frac{\kappa^{\sigma+3 / 2}}{\Gamma(\sigma+3)} K_{\sigma+3 / 2}(\kappa b),
$$


where $\kappa^{2}=\eta^{2}+q^{2}$ and $b=e^{-a}$. Then

$$
J_{m}^{\eta q \sigma}(x)=2(2 \pi)^{3 / 2} b^{3 / 2} \frac{\kappa^{\sigma+3 / 2}}{\Gamma(\sigma+3)} K_{\sigma+3 / 2}(\kappa b) \Psi_{\eta q}^{m}(\xi, z, \varphi) .
$$

It follows from (7.30) that

$$
\begin{aligned}
\psi(x)= & \frac{\mathrm{i} b^{3 / 2}}{(2 \pi)^{5 / 2}} \sum_{m=-\infty}^{\infty} \int_{\delta-\mathrm{i} \infty}^{\delta+\mathrm{i} \infty} d \sigma \frac{\cot \pi \sigma}{\Gamma(\sigma)} \int_{0}^{\infty} d \eta \eta \int_{-\infty}^{\infty} d q A_{m}(\eta, q, \sigma) \\
& \times \kappa^{\sigma+3 / 2} K_{\sigma+3 / 2}(\kappa b) \Psi_{\eta q}^{m}(\xi, z, \varphi),
\end{aligned}
$$

where $\kappa^{2}=\eta^{2}+q^{2}, e^{-a}=b$, and $\Psi_{\eta q}^{m}(\xi, z, \varphi)$ is such function as in (7.27) but determined for other variables.

We have from (7.29) and (6.8) that

$$
A_{m}(\eta, q, \sigma)=\int_{H_{+}^{4}} \frac{d^{4} x}{x_{0}} \psi(x) \int_{\Gamma_{O}} d k^{\prime}\left[x, k^{\prime}\right]^{\sigma} \overline{\Psi_{\eta q}^{m}\left(k^{\prime}\right)} .
$$

Integrating over $k^{\prime}$ we get

$$
A_{m}(\eta, q, \sigma)=2(2 \pi)^{3 / 2} \frac{\kappa^{-\sigma-3 / 2}}{\Gamma(-\sigma)} \int_{H_{+}^{4}} \frac{d^{4} x}{x_{0}} \psi(x) b^{3 / 2} K_{\sigma+3 / 2}(\kappa b) \overline{\Psi_{\eta q}^{m}(\xi, z, \varphi)}
$$

where

$$
d x^{4} / x_{0}=e^{3 a} d a \xi d \xi d z d \varphi .
$$

For the unitary case, when $\sigma=i \rho-3 / 2$ and $\delta=-3 / 2$, formulas (7.31) and (7.32) take the form

$$
\begin{gathered}
\psi(x)=\frac{1}{\pi}\left(\frac{b}{2 \pi}\right)^{3 / 2} \sum_{m=-\infty}^{\infty} \int_{0}^{\infty} d \rho\left(\rho^{2}+1 / 4\right) \frac{\rho \tanh \pi \rho}{\Gamma(\mathrm{i} \rho+3 / 2)} \\
\times \int_{0}^{\infty} d \eta \eta \int_{-\infty}^{\infty} d q A_{m}(\eta, q, \rho) \kappa^{\mathrm{i} \rho} K_{\mathrm{i} \rho}(\kappa b) \Psi_{\eta q}^{m}(\xi, z, \varphi), \\
A_{m}(\eta, q, \rho)=\frac{2(2 \pi)^{3 / 2} \kappa^{-\mathrm{i} \rho}}{\Gamma(-\mathrm{i} \rho+3 / 2)} \int_{H_{+}^{4}} \frac{d^{4} x}{x_{0}} \psi(x) b^{3 / 2} K_{\mathrm{i} \rho}(\kappa b) \overline{\Psi_{\eta q}^{m}(\xi, z, \Phi)} .
\end{gathered}
$$

The formula (7.33) is an expansion of the function $\psi(x)$ in basis elements (5.31). The formulas (7.33) and (7.34) show that the functions (5.31) in a normed form have the form

$$
\Phi_{\rho \eta q}^{m}(b, \xi, z, \varphi)=\frac{1}{\pi \sqrt{2 \pi}} \frac{1}{|\Gamma(\mathrm{i} \rho+3 / 2)|} b^{3 / 2} K_{\mathrm{i} \rho}(\kappa b) J_{m}(\eta \xi) e^{\mathrm{i} q z} e^{\mathrm{i} m \varphi} .
$$

The normalization condition is

$$
\left\langle\Phi_{\rho^{\prime} \eta^{\prime} q^{\prime}}^{m^{\prime}}, \Phi_{\rho \eta q}^{m}\right\rangle=\frac{\delta\left(\rho-\rho^{\prime}\right)}{\rho\left(\rho^{2}+1 / 4\right) \tanh \pi \rho} \frac{\delta\left(\eta-\eta^{\prime}\right)}{\eta} \delta\left(q-q^{\prime}\right) \delta_{m m^{\prime}} .
$$

The Plancherel formula for the transforms (7.33)-(7.34) is

$$
\int_{H_{+}^{4}} \frac{d^{4} x}{x_{0}}|\psi(x)|^{2}=\frac{1}{(2 \pi)^{4}} \sum_{m=-\infty}^{\infty} \int_{0}^{\infty} d \rho\left(\rho^{2}+\frac{1}{4}\right) \rho \tanh \pi \rho \int_{0}^{\infty} d \eta \eta \int_{-\infty}^{\infty} d q\left|A_{m}(\eta, q, \rho)\right|^{2}
$$




\subsection{Expansion in $O T$-coordinate system}

We leave in this case the contour $\Gamma_{O}$ of two previous subsections and introduce on it the coordinates $\chi_{1}, \chi_{2}, \chi_{3}$ :

$$
\begin{array}{ll}
k_{0}^{\prime}=\left(1+\chi^{2}\right) / 2, & k_{1}^{\prime}=\chi_{1}, \quad k_{2}^{\prime}=\chi_{2}, \quad k_{3}^{\prime}=\chi_{3}, \quad k_{4}^{\prime}=\left(-1+\chi^{2}\right) / 2, \\
\chi^{2}=\chi_{1}^{2}+\chi_{2}^{2}+\chi_{3}^{2}, & -\infty<\chi_{i}<\infty, \quad i=1,2,3 .
\end{array}
$$

We consider the expansion of the function $\Psi\left(k^{\prime}, \sigma\right)$ on this contour in functions

$$
\Psi_{\kappa}\left(k^{\prime}\right) \equiv \Psi_{\boldsymbol{\kappa}}(\chi)=(2 \pi)^{-3 / 2} e^{i \kappa \chi}, \quad \kappa=\left(\kappa_{1}, \kappa_{2}, \kappa_{3}\right), \quad \chi=\left(\chi_{1}, \chi_{2}, \chi_{3}\right),
$$

and have

$$
\Psi\left(k^{\prime}, \sigma\right)=\int_{\mathbb{R}^{3}} d \boldsymbol{\kappa} A(\boldsymbol{\kappa}, \sigma) \Psi_{\boldsymbol{\kappa}}\left(k^{\prime}\right) .
$$

The inverse transform is

$$
A(\boldsymbol{\kappa}, \sigma)=\int_{\Gamma_{O}} d k^{\prime} \Psi\left(k^{\prime}, \sigma\right) \overline{\Psi_{\kappa}\left(k^{\prime}\right)}
$$

where $d k^{\prime}=d \boldsymbol{\chi}$. Substituting the expression (7.38) into (6.7) we get

$$
\psi(x)=\frac{\mathrm{i}}{2(2 \pi)^{4}} \int_{\delta-\mathrm{i} \infty}^{\delta+\mathrm{i} \infty} d \sigma \frac{\Gamma(\sigma+3)}{\Gamma(\sigma)} \cot \pi \sigma \int_{\mathbb{R}^{3}} d \boldsymbol{\kappa} A(\boldsymbol{\kappa}, \sigma) \int_{\Gamma_{O}} d k^{\prime}\left[x, k^{\prime}\right]^{-\sigma-3} \Psi_{\boldsymbol{\kappa}}\left(k^{\prime}\right) .
$$

We have to calculate the integral

$$
J^{\kappa \sigma}(x)=\int_{\Gamma_{O}} d k^{\prime}\left[x, k^{\prime}\right]^{-\sigma-3} \Psi_{\kappa}\left(k^{\prime}\right) .
$$

First we consider the integral

$$
J^{\kappa \sigma}(a) \equiv J^{\kappa \sigma}\left(x_{0}(a)\right)=(2 \pi)^{-3 / 2}\left(2 e^{-a}\right)^{\sigma+3} \int_{\mathbb{R}^{3}} d \boldsymbol{\chi}\left(e^{-2 a}+\chi^{2}\right)^{-\sigma-3} e^{i \kappa \chi} .
$$

We use the spherical coordinates. Taking into account that

$$
\int_{0}^{\pi} d \theta \sin \theta e^{\mathrm{i} \kappa \chi \cos \theta}=\left(\frac{2 \pi}{\kappa \chi}\right)^{1 / 2} J_{1 / 2}(\kappa \chi)
$$

where $\theta$ is an angle between $\boldsymbol{\kappa}$ and $\boldsymbol{\chi}$, we have

$$
J^{\kappa \sigma}(a)=\left(2 e^{-a}\right)^{\sigma+3} \kappa^{-1 / 2} \int_{0}^{\infty} d \chi \chi^{3 / 2}\left(e^{-2 a}+\chi^{2}\right)^{-\sigma-3} J_{1 / 2}(\kappa \chi),
$$

where $\kappa=\left(\kappa_{1}^{2}+\kappa_{2}^{2}+\kappa_{3}^{2}\right)^{1 / 2}$. Using formula 6.565(4) in [40], one receives

$$
J^{\kappa \sigma}(a)=2 \kappa^{\sigma+3 / 2} b^{3 / 2} \frac{1}{\Gamma(\sigma+3)} K_{\sigma+3 / 2}(\kappa b), \quad b=e^{-a} .
$$

Since $x=x(a, \mathbf{y})$ is obtained from $x^{0}(a)$ by means of the shift from the subgroup $T(3)$, we get

$$
J^{\kappa \sigma}(\mathbf{x})=2(2 \pi)^{3 / 2} b^{3 / 2} \kappa^{\sigma+3 / 2} \frac{1}{\Gamma(\sigma+3)} K_{\sigma+3 / 2}(\kappa b) \Psi_{\kappa}(\mathbf{y}) .
$$


Thus, we have the following formula for an expansion of the function $\psi(x)$ :

$$
\psi(x)=\frac{\mathrm{i} b^{3 / 2}}{(2 \pi)^{5 / 2}} \int_{\delta-i \infty}^{\delta+\mathrm{i} \infty} d \sigma \frac{\cot \pi \sigma}{\Gamma(\sigma)} \int_{\mathbb{R}^{3}} d \boldsymbol{\kappa} A(\boldsymbol{\kappa}, \sigma) \kappa^{\sigma+3 / 2} K_{\sigma+3 / 2}(\kappa b) \Psi_{\boldsymbol{\kappa}}(\mathbf{y}),
$$

where $b=e^{-a}$. In order to express $A(\boldsymbol{\kappa}, \sigma)$ in terms of $\psi(x)$ we substitute the expression (6.8) for $\Psi\left(k^{\prime}, \sigma\right)$ into (7.39). Then

$$
A(\boldsymbol{\kappa}, \sigma)=\int_{H_{+}^{4}} \frac{d^{4} x}{x_{0}} \psi(x) \int_{\Gamma_{O}} d k^{\prime}\left[x, k^{\prime}\right]^{\sigma} \overline{\Psi_{\boldsymbol{\kappa}}\left(k^{\prime}\right)} .
$$

Performing integration in $k^{\prime}$ over the contour $\Gamma_{O}$, we find

$$
A(\boldsymbol{\kappa}, \sigma)=\frac{2(2 \pi)^{3 / 2}}{\Gamma(-\sigma)} \kappa^{-\sigma-3 / 2} \int_{H_{+}^{4}} \frac{d^{4} x}{x_{0}} \psi(x) b^{3 / 2} K_{\sigma+3 / 2}(\kappa b) \overline{\Psi_{\boldsymbol{\kappa}}(\mathbf{y})},
$$

where

$$
d^{4} x / x_{0}=e^{3 a} d a d \mathbf{y}=e^{3 a} d a d y_{1} d y_{2} d y_{3} .
$$

For the unitary case (when $\sigma=\mathrm{i} \rho-3 / 2$ and $\delta=-3 / 2$ ) one gets

$$
\begin{aligned}
& \psi(x)=\frac{1}{\pi}\left(\frac{b}{2 \pi}\right)^{3 / 2} \int_{0}^{\infty} d \rho\left(\rho^{2}+\frac{1}{4}\right) \frac{\rho \tanh \pi \rho}{\Gamma\left(\mathrm{i} \rho+\frac{3}{2}\right)} \int_{\mathbb{R}^{3}} d \boldsymbol{\kappa} A(\boldsymbol{\kappa}, \rho) \kappa^{\mathrm{i} \rho} K_{\mathrm{i} \rho}(\kappa b) \Psi_{\boldsymbol{\kappa}}(\mathbf{y}), \\
& A(\boldsymbol{\kappa}, \rho)=\frac{2(2 \pi)^{3 / 2}}{\Gamma(-\mathrm{i} \rho+3 / 2)} \kappa^{-\mathrm{i} \rho} \int_{H_{+}^{4}} \frac{d^{4} x}{x_{0}} \psi(x) b^{3 / 2} K_{\mathrm{i} \rho}(\kappa b) \overline{\Psi_{\boldsymbol{\kappa}}(\mathbf{y})} .
\end{aligned}
$$

The formula (7.42) gives an expansion of the function $\psi(x)$ in the basis functions (5.35). A normed form of these basis functions is

$$
\Phi^{\rho \boldsymbol{\kappa}}(b, \mathbf{y})=\frac{1}{2 \pi^{2}} \frac{1}{|\Gamma(\mathrm{i} \rho+3 / 2)|} b^{3 / 2} K_{\mathrm{i} \rho}(\kappa b) e^{\mathrm{i} \kappa \mathbf{y}} .
$$

The normalization condition is

$$
\left\langle\Phi^{\rho^{\prime} \boldsymbol{\kappa}^{\prime}}, \Phi^{\rho \boldsymbol{\kappa}}\right\rangle=\frac{\delta\left(\rho-\rho^{\prime}\right)}{\left(\rho^{2}+1 / 4\right) \rho \tanh \pi \rho} \delta\left(\boldsymbol{\kappa}-\boldsymbol{\kappa}^{\prime}\right) .
$$

The Plancherel formula for the transforms (7.42)-(7.43) is

$$
\int_{H_{+}^{4}} \frac{d^{4} x}{x_{0}}|\psi(x)|^{2}=\frac{1}{(2 \pi)^{4}} \int_{0}^{\infty} d \rho\left(\rho^{2}+\frac{1}{4}\right) \rho \tanh \pi \rho \int_{\mathbb{R}^{3}} d \boldsymbol{\kappa}|A(\boldsymbol{\kappa}, \rho)|^{2} .
$$

\subsection{Expansion in $C$-coordinate system}

In this case we take a cut of the cone $C_{+}^{4}$ by the cylinder $k_{0}^{2}-k_{4}^{2}=1$. This contour is denote by $\Gamma_{C}$. We parametrize this contour by the coordinates $c, \Theta, \Phi$ such that

$$
\begin{aligned}
& k_{0}^{\prime}=\cosh c, \quad k_{1}^{\prime}=\sin \Theta \cos \Phi, \quad k_{2}^{\prime}=\sin \Theta \sin \Phi, \quad k_{3}^{\prime}=\cos \Theta, \quad k_{4}^{\prime}=\sinh c, \\
& -\infty<c<\infty, \quad 0<\Theta \leqslant \pi, \quad 0 \leqslant \Phi<2 \pi .
\end{aligned}
$$

We expand the function $\Psi\left(k^{\prime}, \sigma\right)$ in the spherical functions $Y_{l m}(\Theta, \Phi)$ and fulfil the Fourier transform in the parameter $c$. As a result, we get

$$
\Psi\left(k^{\prime}, \sigma\right)=\sum_{l=0}^{\infty} \sum_{m=-l}^{l} \int_{-\infty}^{\infty} d \tau C_{l m}(\tau, \sigma) e^{\mathrm{i} \tau c} Y_{l m}(\Theta, \Phi) .
$$


The coefficients $C_{l m}(\tau, \sigma)$ are given by

$$
C_{l m}(\tau, \sigma)=\frac{1}{2 \pi} \int_{\Gamma_{C}} d k^{\prime} \Psi\left(k^{\prime}, \sigma\right) e^{-\mathrm{i} \tau c} \overline{Y_{l m}(\Theta, \Phi)}
$$

where $d k^{\prime}=\sin \Theta d c d \Theta d \Phi$. Substituting this expression for the coefficients $C_{l m}(\tau, \sigma)$ into $(6.7)$ one has

$$
\begin{aligned}
\psi(x)= & \frac{\mathrm{i}}{2(2 \pi)^{4}} \sum_{l=0}^{\infty} \sum_{m=-l}^{l} \int_{\delta-\mathrm{i} \infty}^{\delta+\mathrm{i} \infty} d \sigma \frac{\Gamma(\sigma+3)}{\Gamma(\sigma)} \cot \pi \sigma \int_{-\infty}^{\infty} d \tau C_{l m}(\tau, \sigma) \\
& \times \int_{\Gamma_{C}} d k^{\prime}\left[x, k^{\prime}\right]^{-\sigma-3} e^{\mathrm{i} \tau c} Y_{l m}(\Theta, \Phi) .
\end{aligned}
$$

We have to calculate the integral

$$
J_{l m}^{\sigma \tau}(x)=\int_{-\infty}^{\infty} d c e^{\mathrm{i} \tau c} \int_{0}^{\pi} d \Theta \sin \Theta \int_{0}^{2 \pi} d \Phi\left[x, k^{\prime}\right]^{-\sigma-3} Y_{l m}(\Theta, \Phi) .
$$

We do this first for the point $x^{0}=(\cosh a, 0,0, \sinh a, 0)$. The problem is reduced to calculation of the integral

$$
J_{l}^{\sigma \tau}(a)=\lambda \int_{-\infty}^{\infty} d c e^{\mathrm{i} \tau c} \int_{0}^{\pi} d \Theta \sin \Theta(\cosh a \cosh c-\cos \Theta \sinh a)^{-\sigma-3} P_{l}(\cos \Theta),
$$

where $\lambda=\sqrt{(2 l+1) \pi}$. Using the relation

$$
P_{l}(x)=\frac{(-1)^{l}}{2^{l} l !} \frac{d^{l}}{d x^{l}}\left[\left(1-x^{2}\right)^{l}\right], \quad x=\cos \theta,
$$

and integrating $l$ times by parts, one gets

$$
\begin{aligned}
J_{l}^{\sigma \tau}(a)= & \lambda \frac{(-1)^{l} \Gamma(-\sigma-2) \sinh ^{l} a}{\Gamma(-\sigma-l-2) 2^{l} l !} \int_{-\infty}^{\infty} d c e^{\mathrm{i} \tau c} \int_{0}^{\pi} d \Theta(\sin \Theta)^{2 l+1} \\
& \times(\cosh a \cosh c-\cos \Theta \sinh a)^{-\sigma-l-3} .
\end{aligned}
$$

Decomposing $(\cosh a \cosh c-\cos \Theta \sinh a)^{-\sigma-l-3}$ into series by using the formula for the Newton binomial and performing termwise integration we find

$$
J_{l}^{\sigma \tau}(a)=\frac{\pi(2 l+1)^{1 / 2} 2^{\sigma+2} \Gamma(A) \Gamma(B) \tanh ^{l} a}{\Gamma(\sigma+3) \Gamma(D) \cosh ^{\sigma+3} a} F_{1}\left(A, B ; D ; \tanh ^{2} a\right),
$$

where

$$
A=(\sigma+l+\mathrm{i} \tau+3) / 2, \quad B=(\sigma+l-\mathrm{i} \tau+3) / 2, \quad D=l+3 / 2 .
$$

For arbitrary value of $x$ we get

$$
J_{l m}^{\sigma \tau}(x)=\frac{2^{\sigma+3} \pi^{3 / 2}}{\Gamma(\sigma+3)} \frac{\Gamma(A) \Gamma(B) \tanh ^{l} a}{\Gamma(D) \cosh ^{\sigma+3} a}{ }_{2} F_{1}\left(A, B ; D ; \tanh ^{2} a\right) Y_{l m}(\theta \varphi) e^{\mathrm{i} \tau b} .
$$

Now we obtain the following expansion of the function $\psi(x)$ :

$$
\psi(x)=\frac{\mathrm{i}}{(4 \pi)^{5 / 2}} \sum_{l=0}^{\infty} \sum_{m=-l}^{l} \tanh ^{l} a \int_{-\infty}^{\infty} d \tau e^{\mathrm{i} \tau b} \int_{\delta-\mathrm{i} \infty}^{\delta+\mathrm{i} \infty} d \sigma \frac{\cot \pi \sigma}{\Gamma(\sigma)}\left(\frac{2}{\cosh a}\right)^{\sigma+3}
$$




$$
\times C_{l m}(\tau, \sigma) \frac{\Gamma(A) \Gamma(B)}{\Gamma(D)}{ }_{2} F_{1}\left(A, B ; D ; \tanh ^{2} a\right) Y_{l m}(\theta, \varphi) .
$$

In order to express the coefficients $C_{l m}(\tau, \sigma)$ in terms of $\psi(x)$, we substitute the expression (6.8) for $\Phi\left(k^{\prime}, \sigma\right)$ into (7.49):

$$
C_{l m}(\tau, \sigma)=\frac{1}{2 \pi} \int_{H_{+}^{4}} \frac{d^{4} x}{x_{0}} \psi(x) \int_{\Gamma_{C}} d k^{\prime}\left[x, k^{\prime}\right]^{\sigma} e^{-\mathrm{i} \tau c} \overline{Y_{l m}(\Theta, \Phi)} .
$$

Integrating in $c, \Theta$ and $\Phi$ one obtains

$$
\begin{aligned}
C_{l m}(\tau, \sigma)= & \frac{\pi^{1 / 2} \Gamma\left(A^{\prime}\right) \Gamma\left(B^{\prime}\right)}{2 \Gamma\left(D^{\prime}\right) \Gamma(-\sigma)} \int_{H_{+}^{4}} \frac{d^{4} x}{x_{0}} \psi(x) \tanh ^{l} a\left(\frac{2}{\cosh a}\right)^{-\sigma} \\
& \times e^{-\mathrm{i} \tau b}{ }_{2} F_{1}\left(A^{\prime}, B^{\prime} ; D^{\prime} ; \tanh ^{2} a\right) \overline{Y_{l m}(\theta, \varphi)},
\end{aligned}
$$

where

$$
\begin{aligned}
& A^{\prime}=(l-\sigma+\mathrm{i} \tau) / 2, \quad B^{\prime}=(l-\sigma-\mathrm{i} \tau) / 2, \quad D^{\prime}=D=l+3 / 2, \\
& d^{4} x / x_{0}=\sinh ^{3} a \sin \theta d a d b d \theta d \varphi .
\end{aligned}
$$

For the unitary case (when $\sigma=\mathrm{i} \rho-3 / 2$ and $\delta=-3 / 2$ ) we have

$$
\begin{aligned}
\psi(x)= & \frac{1}{(2 \pi)^{5 / 2}} \sum_{l=0}^{\infty} \sum_{m=-l}^{l} \tanh ^{l} a \int_{0}^{\infty} d \rho\left(\rho^{2}+1 / 4\right) \rho \tanh \pi \rho 2^{i \rho}(\cosh a)^{-\mathrm{i} \rho-3 / 2} \\
& \times \int_{-\infty}^{\infty} d \tau e^{\mathrm{i} \tau b} C_{l m}(\tau, \rho) \frac{\Gamma\left(\frac{\mathrm{i} \rho+\mathrm{i} \tau+l+3 / 2}{2}\right) \Gamma\left(\frac{\mathrm{i} \rho-\mathrm{i} \tau+l+3 / 2}{2}\right)}{\Gamma(\mathrm{i} \rho+3 / 2) \Gamma(l+3 / 2)} \\
& \times{ }_{2} F_{1}\left(\frac{\mathrm{i} \rho+\mathrm{i} \tau+l+3 / 2}{2}, \frac{\mathrm{i} \rho-\mathrm{i} \tau+l+3 / 2}{2} ; l+\frac{3}{2} ; \tanh ^{2} a\right) Y_{l m}(\theta, \varphi),
\end{aligned}
$$

and

$$
\begin{aligned}
C_{l m}(\tau, \rho)= & (2 \pi)^{1 / 2} \frac{\Gamma\left(\frac{-\mathrm{i} \rho+\mathrm{i} \tau+l+3 / 2}{2}\right) \Gamma\left(\frac{-\mathrm{i} \rho-\mathrm{i} \tau+l+3 / 2}{2}\right)}{\Gamma(l+3 / 2) \Gamma(-\mathrm{i} \rho+3 / 2)} 2^{-\mathrm{i} \rho} \\
& \times \int_{H_{+}^{4}} \frac{d^{4} x}{x_{0}} \psi(x) \tanh ^{l} a(\cosh a)^{\mathrm{i} \rho-3 / 2} e^{-\mathrm{i} \tau b} \overline{Y_{l m}(\theta, \varphi)} \\
& \times{ }_{2} F_{1}\left(\frac{-\mathrm{i} \rho+\mathrm{i} \tau+l+3 / 2}{2}, \frac{-\mathrm{i} \rho-\mathrm{i} \tau+l+3 / 2}{2} ; l+\frac{3}{2} ; \tanh ^{2} a\right) .
\end{aligned}
$$

It follows from these formulas that the function $\psi(x)$ expands in the basis (5.43) and the normed basis functions are of the form

$$
\begin{aligned}
\Psi_{l m}^{\rho \tau}(a, b, \theta, \varphi)= & \frac{1}{2 \pi} \frac{\left|\Gamma\left(\frac{\mathrm{i} \rho+\mathrm{i} \tau+l+3 / 2}{2}\right) \Gamma\left(\frac{\mathrm{i} \rho-\mathrm{i} \tau+l+3 / 2}{2}\right)\right|}{\Gamma(\mathrm{i} \rho+3 / 2) \Gamma(l+3 / 2)}(\cosh a)^{-3 / 2-\mathrm{i} \rho} \tanh ^{l} a \\
& \times{ }_{2} F_{1}\left(\frac{\mathrm{i} \rho+\mathrm{i} \tau+l+3 / 2}{2}, \frac{\mathrm{i} \rho-\mathrm{i} \tau+l+3 / 2}{2} ; l+\frac{3}{2} ; \tanh ^{2} a\right) e^{\mathrm{i} \tau b} Y_{l m}(\theta, \varphi) .
\end{aligned}
$$

The normalization condition is

$$
\left\langle\Psi_{l^{\prime} m^{\prime}}^{\rho^{\prime} \tau^{\prime}}, \Psi_{l m}^{\rho \tau}\right\rangle=\frac{\delta\left(\rho-\rho^{\prime}\right)}{\rho\left(\rho^{2}+1 / 4\right) \tanh \pi \rho} \delta\left(\tau-\tau^{\prime}\right) \delta_{l l^{\prime}} \delta_{m m^{\prime}} .
$$

The Plancherel formula holds:

$$
\int_{H_{+}^{4}} \frac{d^{4} x}{x_{0}}|\psi(x)|^{2}=\frac{1}{(2 \pi)^{3}} \sum_{l=0}^{\infty} \sum_{m=-l}^{l} \int_{0}^{\infty} d \rho\left(\rho^{2}+1 / 4\right) \rho \tanh \pi \rho \int_{-\infty}^{\infty} d \tau\left|C_{l m}(\tau, \rho)\right|^{2} .
$$




\subsection{Expansion in $\boldsymbol{S H}$-coordinate system}

In this case we take the cut $\Gamma_{S H}$ of $C_{+}^{4}$ by the cylinder $k_{3}^{2}+k_{4}^{2}=1$ as the contour $\Gamma$. This contour is a product of a circle and the higher sheet of the two-sheeted hyperboloid $H_{+}^{2}$. We choose on $\Gamma_{S H}$ the coordinates $c, \alpha, \beta$, where

$$
\begin{array}{ll}
k_{0}^{\prime}=\cosh c, & k_{1}^{\prime}=\sinh c \cos \alpha, \quad k_{2}^{\prime}=\sinh c \sin \alpha, \quad k_{3}^{\prime}=\cos \beta, \quad k_{4}^{\prime}=\sin \beta, \\
0 \leqslant c<\infty, & 0 \leqslant \alpha, \beta<2 \pi .
\end{array}
$$

Let us perform the Fourier transform of the function $\psi\left(k^{\prime}, \sigma\right)$ in the parameter $\beta$. One can consider coefficients of this expansion as functions on $H_{+}^{2}$, parametrized by spherical coordinates $c$ and $\alpha$. We expand these coefficients in basis functions on $H_{+}^{2}$ in spherical coordinates (they correspond to the reduction $\left.S O_{0}(1,2) \supset S O(2)\right)$ using the formulas

$$
\begin{aligned}
& \phi(c, \alpha)=\frac{1}{4 \pi \mathrm{i}} \sum_{m=-\infty}^{\infty} \int_{\varepsilon-\mathrm{i} \infty}^{\varepsilon+\mathrm{i} \infty} d \lambda \lambda \cot \pi \lambda \frac{\Gamma(-\lambda)}{\Gamma(-\lambda+m)} B_{m}(\lambda) P_{-\lambda-1}^{m}(\cosh c) e^{\mathrm{i} m \alpha}, \\
& B_{m}(\lambda)=\frac{\Gamma(\lambda+1)}{\Gamma(\lambda-m+1)} \int_{0}^{\infty} d c \int_{0}^{2 \pi} d \alpha \phi(c, \alpha) P_{\lambda}^{-m}(\cosh c) e^{-\mathrm{i} m \alpha}
\end{aligned}
$$

As a result, the formulas (6.7)-(6.8) take the form

$$
\begin{aligned}
& \psi(x)= \frac{1}{4(2 \pi)^{5}} \sum_{m, m^{\prime}=-\infty}^{\infty} \int_{\delta-\mathrm{i} \infty}^{\delta+\mathrm{i} \infty} d \sigma \frac{\Gamma(\sigma+3)}{\Gamma(\sigma)} \cot \pi \sigma \int_{\varepsilon-\mathrm{i} \infty}^{\varepsilon+\mathrm{i} \infty} d \lambda \lambda \cot \pi \lambda \frac{\Gamma(-\lambda)}{\Gamma(-\lambda+m)} \\
& \times B_{m m^{\prime}}(\lambda, \sigma) \int_{\Gamma_{S H}} d k^{\prime}\left[x, k^{\prime}\right]^{-\sigma-3} P_{-\lambda-1}^{m}(\cosh c) e^{\mathrm{i} m \alpha} e^{\mathrm{i} m^{\prime} \beta}, \\
& B_{m m^{\prime}}(\lambda, \sigma)=\frac{1}{2 \pi} \frac{\Gamma(\lambda+1)}{\Gamma(\lambda+m+1)} \int_{H_{+}^{4}} \frac{d^{4} x}{x_{0}} \int_{\Gamma_{S H}} d k^{\prime}\left[x, k^{\prime}\right]^{\sigma} P_{\lambda}^{m}(\cosh c) e^{-\mathrm{i} m \alpha} e^{-\mathrm{i} m^{\prime} \beta},
\end{aligned}
$$

where $d k^{\prime}=\sinh c d c d \alpha d \beta$. One integrates over $\Gamma_{S H}$ in the same way as in the previous cases. We give a final formula for expansion of the function $\psi(x)$ in basis functions related to unitary representations $\left(\sigma=\mathrm{i} \rho-\frac{3}{2}, \lambda=\mathrm{i} \omega-\frac{1}{2}, 0 \leqslant \rho, \omega<\infty\right)$ :

$$
\begin{aligned}
\psi(x)= & \frac{1}{\pi(4 \pi)^{5 / 2}} \sum_{m^{\prime}=-\infty}^{\infty} \sum_{m=-\infty}^{\infty} \int_{0}^{\infty} d \rho\left(\rho^{2}+\frac{1}{4}\right) \rho \tanh \pi \rho\left(\frac{2}{\cosh a}\right)^{\mathrm{i} \rho+3 / 2} \\
& \times \int_{0}^{\infty} d \omega \omega \tanh \pi \omega \frac{\Gamma\left(-\mathrm{i} \omega+\frac{1}{2}\right) \Gamma\left(\frac{\mathrm{i} \rho-\mathrm{i} \omega+m^{\prime}+1}{2}\right) \Gamma\left(\frac{\mathrm{i} \omega+\mathrm{i} \rho+m^{\prime}+1}{2}\right)}{m^{\prime} ! \Gamma\left(\mathrm{i} \rho+\frac{3}{2}\right) \Gamma\left(-\mathrm{i} \omega+m+\frac{1}{2}\right)} B_{m^{\prime} m}(\omega, \rho) \\
& \times P_{-\mathrm{i} \omega-1 / 2}^{m}(\cosh b) e^{\mathrm{i} m \varphi} e^{\mathrm{i} m^{\prime} \Phi}{ }_{2} F_{1}\left(\frac{\mathrm{i} \rho+\mathrm{i} \omega+m^{\prime}+1}{2}, \frac{\mathrm{i} \rho-\mathrm{i} \omega+m^{\prime}+1}{2} ; m^{\prime}+1 ; \tanh ^{2} a\right),
\end{aligned}
$$

where

$$
\begin{aligned}
B_{m^{\prime} m}(\omega, \rho)= & \frac{\sqrt{\pi} \Gamma\left(\mathrm{i} \omega+\frac{1}{2}\right) \Gamma\left(\frac{-\mathrm{i} \rho+\mathrm{i} \omega+m^{\prime}+1}{2}\right) \Gamma\left(\frac{-\mathrm{i} \rho-\mathrm{i} \omega+m^{\prime}+1}{2}\right)}{2 \Gamma\left(-\mathrm{i} \rho+\frac{3}{2}\right) \Gamma\left(m^{\prime}+1\right) \Gamma\left(\mathrm{i} \omega+m+\frac{1}{2}\right)} \\
& \times \int_{H_{+}^{4}} \frac{d^{4} x}{x_{0}} \psi(x)\left(\frac{2}{\cosh a}\right)^{-\mathrm{i} \rho+3 / 2} \tanh ^{m^{\prime}} a P_{\mathrm{i} \omega-1 / 2}^{m}(\cosh b) e^{-\mathrm{i} m \varphi} e^{-\mathrm{i} m^{\prime} \Phi} \\
& \times{ }_{2} F_{1}\left(\frac{-\mathrm{i} \rho-\mathrm{i} \omega+m^{\prime}+1}{2}, \frac{\mathrm{i} \omega-\mathrm{i} \rho+m^{\prime}+1}{2} ; m^{\prime}+1 ; \tanh ^{2} a\right),
\end{aligned}
$$


and

$$
d^{4} x / x_{0}=\sinh ^{3} a \sinh b d a d b d \varphi d \Phi .
$$

It follows from these formulas that the function $\psi(x)$ expands in the basis (5.49) and the normed basis functions are of the form

$$
\begin{aligned}
\Psi_{\rho \omega \tilde{m} m}(a, b, \varphi, \Phi)= & \frac{1}{(2 \pi)^{3 / 2}} \frac{\left|\Gamma\left(\frac{\mathrm{i} \rho+\mathrm{i} \omega+\tilde{m}+1}{2}\right) \Gamma\left(\frac{\mathrm{i} \rho-\mathrm{i} \omega+\tilde{m}+1}{2}\right)\right|}{\Gamma(\tilde{m}+1)|\Gamma(\mathrm{i} \rho+3 / 2)|} \frac{|\Gamma(i \omega+1 / 2)|}{|\Gamma(\mathrm{i} \omega+m+1 / 2)|} \\
& \times \tanh ^{\tilde{m}} a(\cosh a)^{-\mathrm{i} \rho-3 / 2} e^{\mathrm{i}(m \varphi+\tilde{m} \Phi)} P_{\mathrm{i} \omega-1 / 2}^{m}(\cosh b) \\
& \times{ }_{2} F_{1}\left(\frac{\mathrm{i} \rho+\mathrm{i} \omega+\tilde{m}+1}{2}, \frac{\mathrm{i} \rho-\mathrm{i} \omega+\tilde{m}+1}{2} ; \tilde{m}+1 ; \tanh ^{2} a\right) .
\end{aligned}
$$

The normalization condition is

$$
\left\langle\Psi_{\rho^{\prime} \omega^{\prime} \tilde{m}^{\prime} m^{\prime}}, \Psi_{\rho \omega \tilde{m} m}\right\rangle=\frac{\delta\left(\rho-\rho^{\prime}\right)}{\rho\left(\rho^{2}+1 / 4\right) \tanh \pi \rho} \frac{\delta\left(\omega-\omega^{\prime}\right)}{\omega \tanh \pi \omega} \delta_{\tilde{m} \tilde{m}^{\prime}} \delta_{m m^{\prime}} .
$$

The Plancherel formula for the expansion (7.62)-(7.63) is of the form

$$
\begin{aligned}
\int_{H_{+}^{4}} \frac{d^{4} x}{x_{0}}|\psi(x)|^{2}= & \frac{1}{(2 \pi)^{4}} \sum_{m, m^{\prime}=-\infty}^{\infty} \int_{0}^{\infty} d \rho\left(\rho^{2}+\frac{1}{4}\right) \rho \tanh \pi \rho \\
& \times \int_{0}^{\infty} d \omega \omega \tanh \pi \omega\left|B_{m m^{\prime}}(\omega, \rho)\right|^{2} .
\end{aligned}
$$

\subsection{Expansion in $S$-coordinate system}

In this case, we take the cut $\Gamma_{S}$ of $C_{+}^{4}$ by the plane $k_{0}=1$ as a contour $\Gamma$. This contour is the three-dimensional sphere $S^{3}$. We choose on $\Gamma_{S}$ the spherical coordinates determined by the formulas

$$
\begin{aligned}
& k_{1}^{\prime}=\sin \gamma \sin \Theta \cos \Phi, \quad k_{2}^{\prime}=\sin \gamma \sin \Theta \sin \Phi, \quad k_{3}^{\prime}=\sin \gamma \cos \Theta, \quad k_{4}^{\prime}=\cos \gamma, \\
& 0 \leqslant \gamma, \Theta<\pi, \quad 0 \leqslant \Phi<\pi, \quad d k^{\prime}=\sin ^{2} \gamma \sin \Theta d \gamma d \Theta d \Phi .
\end{aligned}
$$

This case is well-studied (see, for example, [3]). We formulate only the result. The expansion of the function $\psi(x)$ is of the form

$$
\begin{aligned}
\psi(x)= & \frac{1}{(2 \pi)^{2}} \sum_{j=0}^{\infty} \sum_{l=0}^{j} \sum_{m=-l}^{l} \int_{0}^{\infty} d \rho\left(\rho^{2}+\frac{1}{4}\right) \rho \tanh \pi \rho A_{j l m}(\rho) \frac{(-1)^{j} \Gamma\left(-\mathrm{i} \rho-\frac{1}{2}\right)}{\Gamma\left(-\mathrm{i} \rho-j-\frac{1}{2}\right)} \\
& \times(\sinh a)^{-1} P_{\mathrm{i} \rho-\frac{1}{2}}^{-j-1}(\cosh a) Y_{j l m}(\beta, \theta, \varphi),
\end{aligned}
$$

where

$$
A_{j l m}(\rho)=\frac{(2 \pi)^{2}(-1)^{j} \Gamma\left(\mathrm{i} \rho-\frac{1}{2}\right)}{\Gamma\left(\mathrm{i} \rho-j-\frac{1}{2}\right)} \int_{H_{+}^{4}} \frac{d^{4} x}{x_{0}} \psi(x)(\sinh a)^{-1} P_{\mathrm{i} \rho-1 / 2}^{-j-1}(\cosh a) \overline{Y_{j l m}(\beta, \theta, \varphi)} .
$$

Here $Y_{j l m}(\beta, \theta, \varphi)$ is the spherical function on $S^{3}$,

$$
Y_{j l m}(\beta, \theta, \varphi)=\left[\frac{(j+1) \Gamma(j+l+2)}{\Gamma(j-l+1)}\right]^{1 / 2}(\sin \beta)^{-1 / 2} P_{j+1 / 2}^{-l-1 / 2}(\cos \beta) Y_{l m}(\theta, \varphi)
$$


and

$$
d^{4} x / x_{0}=\sinh ^{3} a \sin ^{2} \beta \sin \theta d a d \beta d \theta d \varphi .
$$

Therefore, the functions

$$
\Phi_{\rho j l m}(\alpha, \beta, \theta, \varphi)=\frac{\left|\Gamma\left(\mathrm{i} \rho-\frac{1}{2}\right)\right|}{\left|\Gamma\left(\mathrm{i} \rho-j-\frac{1}{2}\right)\right|} \sinh ^{-1} a P_{\mathrm{i} \rho-1 / 2}^{-j-1}(\cosh a) Y_{j l m}(\beta, \theta, \varphi)
$$

constitute a normed basis in the space $L^{2}\left(H_{+}^{4}\right)$ and normalization condition is

$$
\left\langle\Phi_{\rho^{\prime} j^{\prime} l^{\prime} m^{\prime}}, \Phi_{\rho j l m}\right\rangle=\frac{\delta\left(\rho-\rho^{\prime}\right)}{\rho\left(\rho^{2}+1 / 4\right) \tanh \pi \rho} \delta_{j j^{\prime}} \delta_{l l^{\prime}} \delta_{m m^{\prime}}
$$

The Plancherel formula is of the form

$$
\int_{H_{+}^{4}} \frac{d^{4} x}{x_{0}}|\psi(x)|^{2}=(2 \pi)^{-4} \sum_{j=0}^{\infty} \sum_{l=0}^{j} \sum_{m=-l}^{l} \int_{0}^{\infty} d \rho\left(\rho^{2}+1 / 4\right) \rho \tanh \pi \rho\left|A_{j l m}(\rho)\right|^{2} .
$$

\section{Coordinate systems and generators of $S O_{0}(1,4)$ on the cone}

In this section we consider coordinate systems on the upper sheet $C_{+}^{4}$ of the cone $C^{4}$ in the 4-dimensional Minkowski space, which is determined by the equations

$$
[x, x]=x_{0}^{2}-x_{1}^{2}-x_{2}^{2}-x_{3}^{2}-x_{4}^{2}=0, \quad x_{0}>0 .
$$

The group $S O_{0}(1,4)$ is a transitive group of transformations of $C_{+}^{4}$. We take the point $x^{0}=$ $(1,0,0,0,1)$ of $C_{+}^{4}$. A maximal subgroup of $S O_{0}(1,4)$, whose elements leave this point invariant, is the subgroup $S O(3) \times T(3)$, where $T(3)$ is generated by $E_{i}=P_{i}+N_{i}, i=1,2,3$ (see (3.13)). Thus, the cone $C_{+}^{4}$ is homeomorphic to the coset space $S O_{0}(1,4) /(S O(3) \times T(3))$.

As in the case of the hyperboloid $H_{+}^{4}$, coordinate systems will be determined by expressions of homogeneous coordinates $x_{\mu}, \mu=0,1,2,3,4$, in terms of corresponding angles of a coordinate system under consideration. We restrict ourselves by 7 coordinate systems. They correspond to the same subgroup chains of $S O_{0}(1,4)$ as in the case of the hyperboloid. Coordinate systems on $C_{+}^{4}$ will be denoted by the same symbols as for the hyperboloid $H_{+}^{4}$.

Spherical coordinate system $S$ (coordinates $a, \beta, \theta, \varphi)$ :

$$
\begin{aligned}
& x_{0}=e^{a} / 2, \quad x_{1}=\left(e^{a} / 2\right) \sin \beta \sin \theta \cos \varphi, \quad x_{2}=\left(e^{a} / 2\right) \sin \beta \sin \theta \sin \varphi, \\
& x_{3}=\left(e^{a} / 2\right) \sin \beta \cos \theta, \quad x_{4}=\left(e^{a} / 2\right) \cos \beta, \\
& -\infty<a<\infty, \quad 0 \leqslant \beta, \theta<\pi, \quad 0 \leqslant \varphi<2 \pi .
\end{aligned}
$$

Hyperbolic coordinate system $H$ (coordinates $a, b, \theta, \varphi)$ :

$$
\begin{aligned}
& x_{0}=\left(e^{a} / 2\right) \cosh b, \quad x_{1}=\left(e^{a} / 2\right) \sinh b \sin \theta \cos \varphi, \quad x_{2}=\left(e^{a} / 2\right) \sinh b \sin \theta \sin \varphi, \\
& x_{3}=\left(e^{a} / 2\right) \sinh b \cos \theta, \quad x_{4}=\varepsilon\left(e^{a} / 2\right), \\
& -\infty<a<\infty, \quad 0 \leqslant b<\infty, \quad 0 \leqslant \theta<\pi, \quad 0 \leqslant \varphi<2 \pi, \quad \varepsilon=\operatorname{sign} x_{4}= \pm .
\end{aligned}
$$

Orispherical coordinate system $O$ (coordinates $a, r, \theta, \varphi)$ :

$$
\begin{aligned}
& x_{0}=e^{a}\left(r^{2}+1\right) / 2, \quad x_{1}=e^{a} r \sin \theta \cos \varphi, \quad x_{2}=e^{a} r \sin \theta \sin \varphi, \\
& x_{3}=e^{a} r \cos \theta, \quad x_{4}=e^{a}\left(r^{2}-1\right) / 2, \\
& -\infty<a<\infty, \quad 0 \leqslant r<\infty, \quad 0 \leqslant \theta<\pi, \quad 0 \leqslant \varphi<2 \pi .
\end{aligned}
$$


Cylindric coordinate system $C$ (coordinates $a, b, \theta, \varphi)$ :

$$
\begin{aligned}
& x_{0}=\left(e^{a} / 2\right) \cosh b, \quad x_{1}=\left(e^{a} / 2\right) \sin \theta \cos \varphi, \quad x_{2}=\left(e^{a} / 2\right) \sin \theta \sin \varphi, \\
& x_{3}=\left(e^{a} / 2\right) \cos \theta, \quad x_{4}=\left(e^{a} / 2\right) \sinh b, \\
& -\infty<a<\infty, \quad-\infty<b<\infty, \quad 0 \leqslant \theta<\pi, \quad 0 \leqslant \varphi<2 \pi .
\end{aligned}
$$

Spherically-hyperbolic coordinate system $S H$ (coordinates $a, b, \Phi, \varphi)$ :

$$
\begin{aligned}
& x_{0}=\left(e^{a} / 2\right) \cosh b, \quad x_{1}=\left(e^{a} / 2\right) \sinh b \cos \varphi, \quad x_{2}=\left(e^{a} / 2\right) \sinh b \sin \varphi, \\
& x_{3}=\left(e^{a} / 2\right) \cos \Phi, \quad x_{4}=\left(e^{a} / 2\right) \sin \Phi, \\
& -\infty<a<\infty, \quad 0 \leqslant b<\infty, \quad 0 \leqslant \varphi, \Phi<2 \pi .
\end{aligned}
$$

Orispherically-cylindric coordinate system $O C$ (coordinates $a, \xi, z, \varphi)$ :

$$
\begin{aligned}
& x_{0}=e^{a}\left(\xi^{2}+z^{2}+1\right) / 2, \quad x_{1}=e^{a} \xi \cos \varphi, \quad x_{2}=e^{a} \xi \sin \varphi, \\
& x_{3}=e^{a} \xi, \quad x_{4}=e^{a}\left(\xi^{2}+z^{2}-1\right) / 2, \\
& -\infty<a<\infty, \quad 0 \leqslant \xi<\infty, \quad-\infty<z<\infty, \quad 0 \leqslant \varphi<2 \pi .
\end{aligned}
$$

Orispherically-translational coordinate system $O T$ (coordinates $\left.a, y_{1}, y_{2}, y_{3}\right)$ :

$$
\begin{aligned}
& x_{0}=e^{a}\left(y^{2}+1\right) / 2, \quad x_{1}=e^{a} y_{1}, \quad x_{2}=e^{a} y_{2}, \quad x_{3}=e^{a} y_{3}, \\
& x_{4}=e^{a}\left(y^{2}-1\right) / 2, \quad y^{2}=y_{1}^{2}+y_{2}^{2}+y_{3}^{2}, \\
& -\infty<a<\infty, \quad-\infty<y_{i}<\infty, \quad i=1,2,3 .
\end{aligned}
$$

Since the cone $C_{+}^{4}$ is an asymptotical surface for the hyperboloid $H_{+}^{4}$, the coordinate systems on $C_{+}^{4}$ are asymptotically (at $a \rightarrow \infty$ ) obtained from the corresponding coordinate systems on $H_{+}^{4}$.

We can write down a differential form of infinitesimal operators $J_{\mu \nu}, \mu, \nu=0,1,2,3,4, \mu<\nu$, on the cone $C_{+}^{4}$. For this we use the formulas

$$
J_{r s}=-\mathrm{i}\left(x_{r} \frac{\partial}{\partial x_{s}}-x_{s} \frac{\partial}{\partial x_{r}}\right), \quad J_{0 s}=-\mathrm{i}\left(x_{0} \frac{\partial}{\partial x_{s}}+x_{s} \frac{\partial}{\partial x_{0}}\right),
$$

where $r, s=1,2,3,4$. We substitute into these formulas the expressions for $x_{\mu}, \mu=0,1,2,3,4$, in terms of coordinates of the corresponding system. The results of such calculations can be found in [17], section 2.6. As an example, we give here a differential form for generators of $S O_{0}(1,4)$ in the hyperbolic coordinate system.

$H$-system:

$$
\begin{aligned}
& M_{1}=-\mathrm{i}\left(-\sin \varphi \frac{\partial}{\partial \theta}-\cot \theta \cos \varphi \frac{\partial}{\partial \varphi}\right), \quad M_{2}=-\mathrm{i}\left(\cos \varphi \frac{\partial}{\partial \theta}-\cot \theta \sin \varphi \frac{\partial}{\partial \varphi}\right), \\
& M_{3}=-\mathrm{i} \frac{\partial}{\partial \varphi}, \quad P_{0}=-\mathrm{i} \varepsilon\left(\cosh b \frac{\partial}{\partial a}-\sinh b \frac{\partial}{\partial b}\right), \\
& P_{1}=-\mathrm{i} \varepsilon\left(\sinh b \sin \theta \cos \varphi \frac{\partial}{\partial a}-\cosh b \sin \theta \cos \varphi \frac{\partial}{\partial b}-\frac{\cos \theta \cos \varphi}{\sinh b} \frac{\partial}{\partial \theta}+\frac{\sin \varphi}{\sinh b \sin \theta} \frac{\partial}{\partial \varphi}\right), \\
& P_{2}=-\mathrm{i} \varepsilon\left(\sinh b \sin \theta \sin \varphi \frac{\partial}{\partial a}-\cosh b \sin \theta \sin \varphi \frac{\partial}{\partial b}-\frac{\cos \theta \sin \varphi}{\sinh b} \frac{\partial}{\partial \theta}-\frac{\cos \varphi}{\sinh b \sin \theta} \frac{\partial}{\partial \varphi}\right), \\
& P_{3}=-\mathrm{i} \varepsilon\left(\sinh b \cos \theta \frac{\partial}{\partial a}-\cosh b \cos \theta \frac{\partial}{\partial b}+\frac{\sin \theta}{\sinh b} \frac{\partial}{\partial \theta}\right), \\
& N_{1}=-\mathrm{i}\left(\sin \theta \cos \varphi \frac{\partial}{\partial b}+\operatorname{coth} b \cos \theta \cos \varphi \frac{\partial}{\partial \theta}-\operatorname{coth} b \frac{\sin \varphi}{\sin \theta} \frac{\partial}{\partial \varphi}\right),
\end{aligned}
$$




$$
\begin{aligned}
& N_{2}=-\mathrm{i}\left(\sin \theta \sin \varphi \frac{\partial}{\partial b}+\operatorname{coth} b \cos \theta \sin \varphi \frac{\partial}{\partial \theta}+\operatorname{coth} b \frac{\cos \varphi}{\sin \theta} \frac{\partial}{\partial \varphi}\right), \\
& N_{3}=-\mathrm{i}\left(\cos \theta \frac{\partial}{\partial b}-\operatorname{coth} b \sin \theta \frac{\partial}{\partial \theta}\right) .
\end{aligned}
$$

\section{Invariant operators and their eigenfunctions on the cone}

Our aim in this section is to find basis functions on the cone $C_{+}^{4}$ corresponding to different coordinate systems. As in the case of the hyperboloid $H_{+}^{4}$, these functions are constructed as eigenfunctions of a full system of self-adjoint differential operators on the cone $C_{+}^{4}$, including the Casimir operator $F$ (see formula $(2.7)$ ) of $S O_{0}(1,4)$ and Casimir operators of the corresponding chain of subgroups.

In all coordinate systems on the cone $C_{+}^{4}$ the Casimir operator $F$ is of the form

$$
F=-\frac{\partial^{2}}{\partial a^{2}}-3 \frac{\partial}{\partial a}
$$

This expression can be obtained from the differential form of the operator $F$ in any coordinate system on $H_{+}^{4}$ by the asymptotic limit $a \rightarrow \infty$.

Let us give the rest of self-adjoint operators of a full system in each coordinate system on $C_{+}^{4}$.

S-system.

$$
\begin{aligned}
& \mathbf{M}^{2}+\mathbf{P}^{2}=-\frac{1}{\sin ^{2} \beta}\left(\frac{\partial}{\partial \beta} \sin ^{2} \beta \frac{\partial}{\partial \beta}+\frac{1}{\sin \theta} \frac{\partial}{\partial \theta} \sin \theta \frac{\partial}{\partial \theta}+\frac{1}{\sin ^{2} \theta} \frac{\partial^{2}}{\partial \varphi^{2}}\right), \\
& \mathbf{M}^{2}=-\left(\frac{1}{\sin \theta} \frac{\partial}{\partial \theta} \sin \theta \frac{\partial}{\partial \theta}+\frac{1}{\sin ^{2} \theta} \frac{\partial^{2}}{\partial \varphi^{2}}\right), \quad M_{3}=-i \frac{\partial}{\partial \varphi} .
\end{aligned}
$$

These operators correspond to the chain of subgroups

$$
S O_{0}(1,4) \supset S O(4) \supset S O(3) \supset S O(2) \text {. }
$$

\section{$H$-system.}

$$
\begin{aligned}
& \mathbf{N}^{2}-\mathbf{M}^{2}=-\frac{1}{\sinh ^{2} \beta}\left(\frac{\partial}{\partial b} \sinh ^{2} b \frac{\partial}{\partial b}+\frac{1}{\sin \theta} \frac{\partial}{\partial \theta} \sin \theta \frac{\partial}{\partial \theta}+\frac{1}{\sin ^{2} \theta} \frac{\partial^{2}}{\partial \varphi^{2}}\right), \\
& \mathbf{M}^{2}=-\left(\frac{1}{\sin \theta} \frac{\partial}{\partial \theta} \sin \theta \frac{\partial}{\partial \theta}+\frac{1}{\sin ^{2} \theta} \frac{\partial^{2}}{\partial \varphi^{2}}\right), \quad M_{3}=-i \frac{\partial}{\partial \varphi} .
\end{aligned}
$$

These operators correspond to the chain of subgroups

$$
S O_{0}(1,4) \supset S O_{0}(1,3) \supset S O(3) \supset S O(2) .
$$

\section{$O$-system.}

$$
\begin{aligned}
& \mathbf{E}^{2}=-\left[\frac{\partial^{2}}{\partial r^{2}}+\frac{2}{r} \frac{\partial}{\partial r}+\frac{1}{r^{2}}\left(\frac{\partial^{2}}{\partial \theta^{2}}+\cot \theta \frac{\partial}{\partial \theta}+\frac{1}{\sin ^{2} \theta} \frac{\partial^{2}}{\partial \varphi^{2}}\right)\right], \\
& \mathbf{M}^{2}=-\left(\frac{1}{\sin \theta} \frac{\partial}{\partial \theta} \sin \theta \frac{\partial}{\partial \theta}+\frac{1}{\sin ^{2} \theta} \frac{\partial^{2}}{\partial^{2} \varphi}\right), \quad M_{3}=-i \frac{\partial}{\partial \varphi} .
\end{aligned}
$$

These operators correspond to the chain of subgroups

$$
S O_{0}(1,4) \supset I S O(3) \supset S O(3) \supset S O(2) \text {. }
$$


$O C$-system.

$$
\begin{aligned}
& \mathbf{E}^{2}=-\left(\frac{\partial^{2}}{\partial \xi^{2}}+\frac{1}{\xi} \frac{\partial}{\partial \xi}+\frac{1}{\xi^{2}} \frac{\partial^{2}}{\partial \varphi^{2}}+\frac{\partial^{2}}{\partial z^{2}}\right), \quad E_{3}=-i \frac{\partial}{\partial z} \\
& M_{3}=-i \frac{\partial}{\partial \varphi}, \quad \tilde{\mathbf{E}^{2}} \equiv E_{1}^{2}+E_{2}^{2}=-\left(\frac{\partial^{2}}{\partial \xi^{2}}+\frac{1}{\xi} \frac{\partial}{\partial \xi}+\frac{1}{\xi^{2}} \frac{\partial^{2}}{\partial \varphi^{2}}\right) .
\end{aligned}
$$

These operators correspond to the chain of subgroups

$$
S O_{0}(1,4) \supset I S O(3) \supset I S O(2) \otimes T_{\perp} \supset S O(2) \otimes T_{\perp} .
$$

OT-system.

$$
\mathbf{E}^{2}=-\left(\frac{\partial^{2}}{\partial y_{1}^{2}}+\frac{\partial^{2}}{\partial y_{2}^{2}}+\frac{\partial^{2}}{\partial y_{3}^{2}}\right), \quad E_{j}=-i \frac{\partial}{\partial y_{j}}, \quad j=1,2,3 .
$$

The corresponding chain of subgroups is

$$
S O_{0}(1,4) \supset I S O(3) \supset T(1) \otimes T(2) \otimes T(3) .
$$

$C$-system.

$$
\mathbf{M}^{2}=-\left(\frac{1}{\sin \theta} \frac{\partial}{\partial \theta} \sin \theta \frac{\partial}{\partial \theta}+\frac{1}{\sin ^{2} \theta} \frac{\partial^{2}}{\partial^{2} \varphi}\right), \quad M_{3}=-i \frac{\partial}{\partial \varphi}, \quad P_{0}=-i \frac{\partial}{\partial b} .
$$

These operators correspond to the chain of subgroups

$$
S O_{0}(1,4) \supset S O_{0}(1,1) \otimes S O(3) \supset S O(2) .
$$

\section{$S H$-system.}

$$
\begin{aligned}
& N_{1}^{2}+N_{2}^{2}-M_{3}^{2}=-\left(\frac{\partial^{2}}{\partial b^{2}}+\operatorname{coth} b \frac{\partial}{\partial b}+\frac{1}{\sinh ^{2} b} \frac{\partial^{2}}{\partial^{2} \varphi}\right), \\
& M_{3}=-i \frac{\partial}{\partial \varphi}, \quad P_{3}=-i \frac{\partial}{\partial \Phi} .
\end{aligned}
$$

The corresponding chain of subgroups is

$$
S O_{0}(1,4) \supset S O_{0}(1,2) \otimes S O^{\prime}(2) \supset S O(2), \quad S O(2) \sim S O^{\prime}(2),
$$

(the subgroup $S O(2)$ is embedded into $S O_{0}(1,2)$ ).

As we see from the expressions for invariant operators in each coordinate system given above their differential form (except for the operator $F$ ) coincides with the corresponding differential form in the corresponding coordinate system on the hyperboloid $H_{+}^{4}$.

It is known (see [3], Chapter 10) that the quasi-regular representation of $S O_{0}(1,4)$ on the Hilbert space $L^{2}\left(C_{+}^{4}\right)$ decomposes into a direct integral of irreducible unitary representations of this group, and each of these irreducible representations is contained in the decomposition twice. (Let us recall that the quasi-regular representation of $S O_{0}(1,4)$ on the Hilbert space $L^{2}\left(H_{+}^{4}\right)$ decomposes into a direct integral of irreducible unitary representations of this group, and each of them is contained in the decomposition once.) Therefore, the spectrum of each full collection of self-adjoint operators in $L^{2}\left(C_{+}^{4}\right)$ given above differs from the corresponding spectrum on $L^{2}\left(H_{+}^{4}\right)$ only by the fact that multiplicity of each eigenvalue is doubled.

As in the case of functions on the hyperboloid, we try to find eigenfunctions of the collections of self-adjoint differential operators on $C_{+}^{4}$ in the form of separated variables. As we have said, 
differential forms of invariant operators (except for the operator $F$ ) for $H_{+}^{4}$ and for $C_{+}^{4}$ coincide in the same type of coordinate systems. For this reason, the corresponding solutions for them are the same. Thus, in order to find eigenfunctions of full collections of self-adjoint operators we have to solve the equation

$$
\left(\frac{\partial^{2}}{\partial a^{2}}+3 \frac{\partial}{\partial a}\right)\langle a \mid \sigma\rangle^{\prime}=\sigma(\sigma+3)\langle a \mid \sigma\rangle^{\prime},
$$

corresponding to the operator $F$. For each fixed complex value of $\sigma$, there are two corresponding linearly independent solutions of this equation:

$$
\langle a \mid \sigma\rangle_{1}^{\prime}=\exp \sigma a, \quad\langle a \mid \sigma\rangle_{2}^{\prime}=\exp (-\sigma-3) a .
$$

In particular, for values of $\sigma$, corresponding to the principal unitary series representations of $S O_{0}(1,4)$ (that is, for $\left.\sigma=\mathrm{i} \rho-\frac{3}{2}, \rho \in \mathbb{R}\right)$, we have the solutions

$$
\langle a \mid \rho\rangle \equiv\left\langle a \mid-\frac{3}{2}+i \rho\right\rangle^{\prime}=\exp \left(-\frac{3}{2} \pm i \rho\right) a .
$$

Thus, a full system of eigenfunctions of a collection of self-adjoint operators, corresponding to a fixed coordinate system, is given by the formula

$$
\Phi_{\rho \gamma}(a, \alpha) \equiv\langle a, \alpha \mid \rho, \gamma\rangle=\langle a \mid \rho\rangle\langle\alpha \mid \gamma\rangle,
$$

where $\alpha$ is a collection of all coordinates except for the coordinate $a, \gamma$ are eigenvalues of all operators except for the operator $F$, and $\langle\alpha \mid \gamma\rangle$ are eigenfunctions of these operators.

It is evident that the function (9.2) is homogeneous in $e^{a}$ of homogeneity degree $(-3 / 2 \pm \mathrm{i} \rho)$. Therefore, the basis functions (9.3) are homogeneous in $e^{a}$.

The orispherical transform (6.3) maps $L^{2}\left(H_{+}^{4}\right)$ not upon the whole space $L^{2}\left(C_{+}^{4}\right)$, but rather upon its subspace (we denote it by $L_{0}^{2}\left(C_{+}^{4}\right)$ ). Indeed, if this transform would map $L^{2}\left(H_{+}^{4}\right)$ upon the whole space $L^{2}\left(C_{+}^{4}\right)$, then multiplicities of irreducible representations of $S O_{0}(1,4)$ in $L^{2}\left(C_{+}^{4}\right)$ would exceed those in $L^{2}\left(H_{+}^{4}\right)$; this cannot be true.

Basis functions of the space $L^{2}\left(C_{+}^{4}\right)$, corresponding to a fixed coordinate system, can be found in different ways. One of these ways is making orispherical transform of the corresponding basis functions of $L^{2}\left(H_{+}^{4}\right)$. However, for most coordinate systems this transform leads to divergent integrals (let us remind that our basis functions are normed to the delta-function). For this reason, we choose another way. As we have said, in the homogeneous coordinates on $H_{+}^{4}$ and on $C_{+}^{4}$ coordinates and differential operators of the operator $F$ and other operators coincide in the asymptotics $a \rightarrow \infty$. The corresponding basis functions on $H_{+}^{4}$ and on $C_{+}^{4}$ also coincide in this asymptotics. The part $\Phi_{\rho}(a)$ of a basis function of $L_{0}^{2}\left(C_{+}^{4}\right)$, which depends on $a$, is a linear combination of the functions $\exp \sigma a$ and $\exp (-\sigma-3) a, \sigma=i \rho-3 / 2$ :

$$
\Phi_{\rho}(a)=C_{1} \exp \sigma a+C_{2} \exp (-\sigma-3) a,
$$

where $C_{1}$ and $C_{2}$ are independent of $a$. The coefficients $C_{1}$ and $C_{2}$ can be found using the fact that the function $\Phi_{\rho}(a)$ and the corresponding basis function on the hyperboloid $H_{+}^{4}$ coincide in the asymptotics $a \rightarrow \infty$. As an example, let us consider the case of the spherical coordinate system.

We take the function $(\sinh a)^{-1} P_{\mathrm{i} \rho-1 / 2}^{-j-1}(\cosh a)$ of $a$, entering into the expression for the basis function $\Phi_{\rho j l m}(a, \beta, \theta, \varphi)$ of the space $L^{2}\left(H_{+}^{4}\right)$ (see formula (7.67)). Expressing the function $P_{\mathrm{i} \rho-1 / 2}^{-j-1}(\cosh a)$ in terms of the Gauss hypergeometric function and using the formula (15.3.6) in [41], we find that for large $a$ the following asymptotics holds:

$$
(\sinh a)^{-1} P_{\mathrm{i} \rho-1 / 2}^{-j-1}(\cosh a) \simeq \frac{2}{\sqrt{\pi}}\left[\frac{\Gamma(\mathrm{i} \rho) \exp (-3 / 2+\mathrm{i} \rho) a}{\Gamma(\mathrm{i} \rho+j+3 / 2)}+\frac{\Gamma(-\mathrm{i} \rho) \exp (-3 / 2-\mathrm{i} \rho) a}{\Gamma(-\mathrm{i} \rho+j+3 / 2)}\right] .
$$


Therefore, for large $a$ the function $\Phi_{\rho j l m}(a, \beta, \theta, \varphi)$ has the form

$$
\begin{aligned}
\Phi_{\rho j l m}(a, \beta, \theta, \varphi) \simeq & \frac{2|\Gamma(\mathrm{i} \rho-1 / 2)|}{\sqrt{\pi}|\Gamma(\mathrm{i} \rho-j-1 / 2)|} Y_{j l m}(\beta, \theta, \varphi) \\
& \times\left[\frac{\Gamma(\mathrm{i} \rho) \exp (-3 / 2+\mathrm{i} \rho) a}{\Gamma(\mathrm{i} \rho+j+3 / 2)}+\frac{\Gamma(-i \rho) \exp (-3 / 2-\mathrm{i} \rho) a}{\Gamma(-\mathrm{i} \rho+j+3 / 2)}\right] .
\end{aligned}
$$

Then

$$
C_{1,2} \equiv C_{ \pm}=\frac{2}{\sqrt{\pi}} \frac{\left|\Gamma\left(\mathrm{i} \rho-\frac{1}{2}\right)\right|}{\left|\Gamma\left(\mathrm{i} \rho-j-\frac{1}{2}\right)\right|} \frac{\Gamma( \pm \mathrm{i} \rho)}{\Gamma\left( \pm \mathrm{i} \rho+j+\frac{3}{2}\right)} .
$$

Thus, in the $S$-coordinate system the basis functions of the space $L_{0}^{2}\left(C_{+}^{4}\right)$, corresponding to the basis functions on $L^{2}\left(H_{+}^{4}\right)$, are of the form

$$
\begin{aligned}
\Phi_{\rho j l m}^{\prime}(a, \beta, \theta, \varphi)= & \frac{2}{\sqrt{\pi}} \frac{|\Gamma(\mathrm{i} \rho-1 / 2)|}{|\Gamma(\mathrm{i} \rho-j-1 / 2)|} Y_{j l m}(\beta, \theta, \varphi) \\
& \times\left[\frac{\Gamma(\mathrm{i} \rho)}{\Gamma(\mathrm{i} \rho+j+3 / 2)} e^{(-3 / 2+\mathrm{i} \rho) a}+\frac{\Gamma(-\mathrm{i} \rho)}{\Gamma(-\mathrm{i} \rho+j+3 / 2)} e^{(-3 / 2-\mathrm{i} \rho) a}\right] .
\end{aligned}
$$

The domains of definition for the indices $\rho, j, l, m$ are the same as in the case of the space $L^{2}\left(H_{+}^{4}\right)$. This remark is true for other coordinate systems considered below.

An explicit form of basis functions in other coordinate systems can be found analogously. Let us write down basis functions on the cone $C_{+}^{4}$ in all the coordinate systems, normed by a delta-function.

\section{$S$-system.}

$$
\Phi_{\rho j l m}(a, \beta, \theta, \varphi) \equiv\langle a, \beta, \theta, \varphi \mid \rho, j, l, m\rangle=\left(C_{S}^{+} e^{(\mathrm{i} \rho-3 / 2) a}+C_{S}^{-} e^{(-\mathrm{i} \rho-3 / 2) a}\right) Y_{j l m}(\beta, \theta, \varphi),
$$

where

$$
C_{S}^{ \pm}=\frac{\sqrt{2}|\Gamma(\mathrm{i} \rho+j+3 / 2)| \Gamma( \pm \mathrm{i} \rho)}{\sqrt{\pi}|\Gamma(\mathrm{i} \rho+3 / 2)| \Gamma( \pm \mathrm{i} \rho+j+3 / 2)} .
$$

The orthogonality relation has the form

$$
\left\langle\Phi_{\rho^{\prime} j^{\prime} l^{\prime} m^{\prime}}, \Phi_{\rho j l m}\right\rangle=\int_{C_{+}^{4}} \frac{d^{4} x}{x_{0}} \Phi_{\rho^{\prime} j^{\prime} l^{\prime} m^{\prime}}(a, \beta, \theta, \varphi) \overline{\Phi_{\rho j l m}(a, \beta, \theta, \varphi)}=\frac{\delta\left(\rho-\rho^{\prime}\right) \delta_{j j^{\prime}} \delta_{l l^{\prime}} \delta_{m m^{\prime}}}{\rho\left(\rho^{2}+1 / 4\right) \tanh \pi \rho},
$$

where $d^{4} x / x_{0}=\frac{1}{8} e^{3 a} \sin ^{2} \beta \sin \theta d a d \beta d \theta d \varphi$.

\section{$H$-system.}

$$
\begin{aligned}
\Phi_{\rho \nu l m}^{ \pm}(a, \beta, \theta, \varphi) & \equiv\langle a, \beta, \theta, \varphi \mid \rho, \nu, l, m\rangle \\
& =\left(C_{H_{ \pm}}^{+} e^{(-3 / 2+\mathrm{i} \rho) a}+C_{H_{ \pm}}^{-} e^{(-3 / 2-\mathrm{i} \rho) a}\right) V_{\nu l m}(\beta, \theta, \varphi),
\end{aligned}
$$

where

$$
\begin{aligned}
& V_{\nu l m}(\beta, \theta, \varphi)=\frac{|\Gamma(\mathrm{i} \nu)|}{|\Gamma(\mathrm{i} \nu-l)|}(\sinh b)^{-1 / 2} P_{\mathrm{i} \nu-1 / 2}^{-l-1 / 2}(\cosh b) Y_{l m}(\theta, \varphi), \\
& C_{H_{ \pm}}^{+}=\frac{\sqrt{2}|\Gamma(\mathrm{i} \rho+\mathrm{i} \nu+1 / 2) \Gamma(\mathrm{i} \rho-\mathrm{i} \nu+1 / 2)| \Gamma(\mathrm{i} \rho)}{\sqrt{\pi}|\Gamma(\mathrm{i} \rho+3 / 2)| \Gamma(\mathrm{i} \rho+\mathrm{i} \nu+1 / 2) \Gamma(\mathrm{i} \rho-\mathrm{i} \nu+1 / 2)}, \\
& C_{H_{ \pm}}^{-}=\frac{\sqrt{2}|\Gamma(\mathrm{i} \rho+\mathrm{i} \nu+1 / 2) \Gamma(\mathrm{i} \rho-\mathrm{i} \nu+1 / 2)|}{\sqrt{\pi}|\Gamma(\mathrm{i} \rho+3 / 2)|}\left(\frac{\cosh \pi \rho}{\pi} \Gamma(-\mathrm{i} \rho) \pm \frac{1}{\Gamma(\mathrm{i} \rho+1)}\right) .
\end{aligned}
$$


The orthogonality relation for the functions (9.7) is

$$
\begin{aligned}
\left\langle\Phi_{\rho^{\prime} \nu^{\prime} l^{\prime} m^{\prime}}^{ \pm}, \Phi_{\rho \nu l m}^{ \pm}\right\rangle & =\int_{C_{+}^{4}} \frac{d^{4} x}{x_{0}} \Phi_{\rho^{\prime} \nu^{\prime} l^{\prime} m^{\prime}}^{ \pm}(a, \beta, \theta, \varphi) \overline{\Phi_{\rho \nu l m}^{ \pm}(a, \beta, \theta, \varphi)} \\
& =\frac{\delta\left(\rho-\rho^{\prime}\right)}{\rho\left(\rho^{2}+1 / 4\right) \tanh \pi \rho} \frac{\delta\left(\nu-\nu^{\prime}\right)}{\nu^{2}} \delta_{l l^{\prime}} \delta_{m m^{\prime}}, \\
\left\langle\Phi_{\rho^{\prime} \nu^{\prime} l^{\prime} m^{\prime}}^{ \pm}, \Phi_{\rho \nu l m}^{\mp}\right\rangle & =0,
\end{aligned}
$$

where $d^{4} x / x_{0}=\left(e^{3 a} / 8\right) \sinh ^{2} b \sin \theta d a d b d \theta d \varphi$.

\section{$O$-system.}

$$
\Phi_{l m}^{\rho \kappa}(a, r, \theta, \varphi) \equiv\langle a, r, \theta, \varphi \mid \rho, \kappa, l, m\rangle=\left(C_{O}^{+} e^{(-3 / 2+\mathrm{i} \rho) a}+C_{O}^{-} e^{(-3 / 2-\mathrm{i} \rho) a}\right) J_{\kappa l m}(r, \theta, \varphi),
$$

where

$$
J_{\kappa l m}(r, \theta, \varphi)=(\kappa r)^{-1 / 2} J_{l+1 / 2}(\kappa r) Y_{l m}(\theta, \varphi), \quad C_{O}^{ \pm}=\frac{(\kappa / 2)^{\mp \mathrm{i} \rho} \Gamma( \pm \mathrm{i} \rho)}{2 \sqrt{\pi}|\Gamma(\mathrm{i} \rho-3 / 2)|} .
$$

The orthogonality relation for the functions (9.8) is

$$
\begin{aligned}
\left\langle\Phi_{l^{\prime} m^{\prime}}^{\rho^{\prime} \kappa^{\prime}}, \Phi_{l m}^{\rho \kappa}\right\rangle & =\int_{C_{+}^{4}} \frac{d^{4} x}{x_{0}} \Phi_{l^{\prime} m^{\prime}}^{\rho^{\prime} \kappa^{\prime}}(a, r, \theta, \varphi) \overline{\Phi_{l m}^{\rho \kappa}(a, r, \theta, \varphi)} \\
& =\frac{\delta\left(\rho-\rho^{\prime}\right)}{\rho\left(\rho^{2}+1 / 4\right) \tanh \pi \rho} \frac{\delta\left(\kappa-\kappa^{\prime}\right)}{\kappa^{2}} \delta_{l l^{\prime}} \delta_{m m^{\prime}},
\end{aligned}
$$

where $d^{4} x / x_{0}=e^{3 a} r^{2} \sin \theta d a d r d \theta d \varphi$.

$O C$-system.

$$
\Phi_{\rho \eta q}^{m}(a, \xi, z, \varphi) \equiv\langle a, \xi, z, \varphi \mid \rho, \eta, q, m\rangle=\left(C_{O C}^{+} e^{(-3 / 2+\mathrm{i} \rho) a}+C_{O C}^{-} e^{(-3 / 2-\mathrm{i} \rho) a}\right) \Psi_{\eta q}^{m}(\xi, z, \varphi),(9
$$

where $q^{2}+\eta^{2}=\kappa^{2}$ and

$$
\Psi_{\eta q}^{m}(\xi, z, \varphi)=\frac{1}{2 \pi} J_{m}(\eta \xi) e^{i q z} e^{i m \varphi}, \quad C_{O C}^{ \pm}=C_{O}^{ \pm}=\frac{(\kappa / 2)^{\mp \mathrm{i} \rho} \Gamma( \pm \mathrm{i} \rho)}{2 \sqrt{\pi}|\Gamma(\mathrm{i} \rho+3 / 2)|} .
$$

The orthogonality relation for the functions (9.9) is

$$
\begin{aligned}
\left\langle\Phi_{\rho^{\prime} \eta^{\prime} q^{\prime}}^{m^{\prime}}, \Phi_{\rho \eta q}^{m}\right\rangle & =\int_{C_{+}^{4}} \frac{d^{4} x}{x_{0}} \Phi_{\rho^{\prime} \eta^{\prime} q^{\prime}}^{m^{\prime}}(a, \xi, z, \varphi) \overline{\Phi_{\rho \eta q}^{m}(a, \xi, z, \varphi)} \\
& =\frac{\delta\left(\rho-\rho^{\prime}\right)}{\rho\left(\rho^{2}+1 / 4\right) \tanh \pi \rho} \frac{\delta\left(\eta-\eta^{\prime}\right)}{\eta} \delta\left(q-q^{\prime}\right) \delta_{m m^{\prime}}
\end{aligned}
$$

where $d^{4} x / x_{0}=e^{3 a} \xi d a d \xi d z d \varphi$.

OT-system.

$$
\Phi^{\rho \kappa}(a, \mathbf{y}) \equiv\langle a, \mathbf{y}, \mid \rho, \boldsymbol{\kappa}\rangle=\left(C_{O T}^{+} e^{(-3 / 2+\mathrm{i} \rho) a}+C_{O T}^{-} e^{(-3 / 2-\mathrm{i} \rho) a}\right) \Psi_{\boldsymbol{\kappa}}(\mathbf{y}),
$$

where

$$
\begin{aligned}
& \Psi_{\boldsymbol{\kappa}}(\mathbf{y})=(2 \pi)^{-3 / 2} \exp (\mathrm{i} \boldsymbol{\kappa y}), \\
& C_{O T}^{ \pm}=C_{O}^{ \pm}=\frac{(\kappa / 2)^{\mp \mathrm{i} \rho} \Gamma( \pm i \rho)}{2 \sqrt{\pi}|\Gamma(\mathrm{i} \rho+3 / 2)|}, \quad \kappa=|\boldsymbol{\kappa}| .
\end{aligned}
$$


The orthogonality relation for the functions (9.10) is

$$
\left\langle\Phi^{\rho^{\prime} \boldsymbol{\kappa}^{\prime}}, \Phi^{\rho \boldsymbol{\kappa}}\right\rangle=\int_{C_{+}^{4}} \frac{d^{4} x}{x_{0}} \Phi^{\rho^{\prime} \boldsymbol{\kappa}^{\prime}}(a, \mathbf{y}) \overline{\Phi^{\rho \boldsymbol{\kappa}}(a, \mathbf{y})}=\frac{\delta\left(\rho-\rho^{\prime}\right) \delta\left(\boldsymbol{\kappa}-\boldsymbol{\kappa}^{\prime}\right)}{\rho\left(\rho^{2}+1 / 4\right) \tanh \pi \rho},
$$

where $d^{4} x / x_{0}=e^{3 a} d a d \mathbf{y}$.

$C$-system.

$$
\Psi_{l, m}^{\rho \tau}(a, b, \theta, \varphi) \equiv\langle a, b, \theta, \varphi \mid \rho, \tau, l, m\rangle=\left(C_{C}^{+} e^{(-3 / 2+i \rho) a}+C_{C}^{-} e^{(-3 / 2-i \rho) a}\right) Y_{l m}^{\tau}(b, \theta, \varphi),
$$

where

$$
\begin{aligned}
& Y_{l m}^{\tau}(b, \theta, \varphi)=(2 \pi)^{-1 / 2} \exp (\mathrm{i} \tau b) Y_{l m}(\theta, \varphi), \\
& C_{C}^{ \pm}=\frac{\sqrt{2}|\Gamma[(\mathrm{i} \rho+\mathrm{i} \tau+l+3 / 2) / 2] \Gamma[(\mathrm{i} \rho-\mathrm{i} \tau+l+3 / 2) / 2]| 2^{\mp \mathrm{i} \rho} \Gamma( \pm \mathrm{i} \rho)}{\sqrt{\pi}|\Gamma(\mathrm{i} \rho+3 / 2)| \Gamma[( \pm \mathrm{i} \rho+\mathrm{i} \tau+l+3 / 2) / 2] \Gamma[( \pm \mathrm{i} \rho-\mathrm{i} \tau+l+3 / 2) / 2]} .
\end{aligned}
$$

The orthogonality relation for the functions (9.11) is

$$
\begin{aligned}
\left\langle\Psi_{l^{\prime} m^{\prime}}^{\rho^{\prime} \tau^{\prime}}, \Psi_{l m}^{\rho \tau}\right\rangle & =\int_{C_{+}^{4}} \frac{d^{4} x}{x_{0}} \Psi_{l^{\prime} m^{\prime}}^{\rho^{\prime} \tau^{\prime}}(a, b, \theta, \varphi) \overline{\Psi_{l m}^{\rho \tau}(a, b, \theta, \varphi)} \\
& =\frac{\delta\left(\rho-\rho^{\prime}\right)}{\rho\left(\rho^{2}+1 / 4\right) \tanh \pi \rho} \delta\left(\tau-\tau^{\prime}\right) \delta_{l l^{\prime}} \delta_{m m^{\prime}}
\end{aligned}
$$

where $d^{4} x / x_{0}=\frac{1}{8} e^{3 a} \sin \theta d a d b d \theta d \varphi$.

$\mathrm{SH}$-system.

$$
\begin{aligned}
\Psi_{\rho \omega m \tilde{m}}(a, b, \varphi, \Phi) & \equiv\langle a, b, \varphi, \Phi \mid \rho, \omega, m, \tilde{m}\rangle \\
& =\left(C_{S H}^{+} e^{(-3 / 2+\mathrm{i} \rho) a}+C_{S H}^{-} e^{(-3 / 2-\mathrm{i} \rho) a}\right) W_{m \tilde{m}}^{\omega}(b, \varphi, \Phi),
\end{aligned}
$$

where

$$
\begin{aligned}
& W_{m \tilde{m}}^{\omega}(b, \varphi, \Phi)=\frac{1}{2 \pi} \frac{|\Gamma(\mathrm{i} \omega+1 / 2)|}{|\Gamma(\mathrm{i} \omega+m+1 / 2)|} P_{\mathrm{i} \omega-1 / 2}^{m}(\cosh b) e^{\mathrm{i}(m \varphi+\tilde{m} \Phi)}, \\
& C_{S H}^{ \pm}=\frac{\sqrt{2}|\Gamma[(\mathrm{i} \rho+\mathrm{i} \omega+\tilde{m}+1) / 2] \Gamma[(\mathrm{i} \rho-\mathrm{i} \omega+\tilde{m}+1) / 2]| 2^{\mp \mathrm{i} \rho} \Gamma( \pm \mathrm{i} \rho)}{\sqrt{\pi}|\Gamma(\mathrm{i} \rho+3 / 2)| \Gamma[( \pm \mathrm{i} \rho+\mathrm{i} \omega+\tilde{m}+1) / 2] \Gamma[( \pm \mathrm{i} \rho-\mathrm{i} \omega+\tilde{m}+1) / 2]} .
\end{aligned}
$$

The orthogonality relation for these basis functions is

$$
\begin{aligned}
\left\langle\Psi_{\rho^{\prime} \omega^{\prime} m^{\prime} \tilde{m}^{\prime}}, \Psi_{\rho \omega m \tilde{m}}\right\rangle & =\int_{C_{+}^{4}} \frac{d^{4} x}{x_{0}} \Psi_{\rho^{\prime} \omega^{\prime} m^{\prime} \tilde{m}^{\prime}}(a, b, \varphi, \Phi) \overline{\Psi_{\rho \omega m \tilde{m}}(a, b, \varphi, \Phi)} \\
& =\frac{\delta\left(\rho-\rho^{\prime}\right)}{\rho\left(\rho^{2}+1 / 4\right) \tanh \pi \rho} \frac{\delta\left(\omega-\omega^{\prime}\right)}{\omega \tanh \pi \omega} \delta_{m m^{\prime}} \delta_{\tilde{m} \tilde{m^{\prime}}},
\end{aligned}
$$

where $d^{4} x / x_{0}=\frac{1}{8} e^{3 a} \sinh b d a d b d \varphi d \Phi$.

Transition coefficients. In applications of harmonic analysis on the hyperboloid and on the cones, it is necessary sometimes to go from expansion of functions $\psi(x), x \in H_{+}^{4}$ (or $x \in C_{+}^{4}$ ), in basis elements in a certain coordinate system to expansion of this function in basis elements in another coordinate system. This transition is fulfilled by means of matrix elements (kernels) of the corresponding transition operator. These matrix elements (kernels) are called coefficients of the transition. 
If we denote a basis function in a coordinate system $A$ by $\langle x \mid \gamma\rangle$ and in a coordinate system $B$ by $\langle x \mid \mu\rangle$, where $x \in H_{+}^{4}\left(\right.$ or $\left.x \in C_{+}^{4}\right)$, then $\langle x \mid \gamma\rangle$ and $\langle x \mid \mu\rangle$ are connected by the formula

$$
\langle x \mid \mu\rangle=\int d \nu(\gamma)\langle x \mid \gamma\rangle\langle\gamma \mid \mu\rangle
$$

where $d \nu(\gamma)$ is the measure in continuous parameters and a sum in discrete parameters in $\gamma$ (this measure coincides with the measure with respect to which the expansion of the function $\psi(x)$ in basis functions $\langle x \mid \gamma\rangle$ is made. If $\langle\gamma \mid \mu\rangle$ is continuous function in variables $\gamma$, then according to orthogonality properties of the functions $\langle x \mid \gamma\rangle$ we have

$$
\langle\gamma \mid \mu\rangle=\int \frac{d^{4} x}{x_{0}}\langle\gamma \mid x\rangle\langle x \mid \mu\rangle, \quad\langle\gamma \mid x\rangle=\overline{\langle x \mid \gamma\rangle}
$$

where integration is over the hyperboloid or the cone.

The quasi-regular representations of the group $S O_{0}(1,4)$ are realized on the spaces $L^{2}\left(H_{+}^{4}\right)$ and $L_{0}^{2}\left(C_{+}^{4}\right)$. These representations are unitary equivalent and the equivalence is given by the orispherical transform (6.3). Besides, for each coordinate system there exist basis functions of these spaces, which are also connected by the orispherical transform (6.3). Therefore, coefficients of transition from one basis to another in $L^{2}\left(H_{+}^{4}\right)$ coincide with the corresponding coefficients of transition in the space $L_{0}^{2}\left(C_{+}^{4}\right)$,

$$
\int_{H_{+}^{4}} \frac{d^{4} x}{x_{0}}\langle\gamma \mid x\rangle\langle x \mid \mu\rangle=\int_{C_{+}^{4}} \frac{d^{4} x}{x_{0}}(O\langle\gamma \mid x\rangle)(O\langle x \mid \mu\rangle),
$$

where $O$ is the operator of the orispherical transform. Thus, we do not need to derive coefficients of transition in $L^{2}\left(H_{+}^{4}\right)$ and in $L_{0}^{2}\left(C_{+}^{4}\right)$. Since basis functions on $L_{0}^{2}\left(C_{+}^{4}\right)$ are simpler than basis functions on $L^{2}\left(H_{+}^{4}\right)$, these coefficients usually derive for the space $L_{0}^{2}\left(C_{+}^{4}\right)$.

\section{Information on semisimple Lie groups and Lie algebras}

Let $G$ be a connected linear (matrix) noncompact real semisimple Lie group and let $\mathfrak{g}$ be its Lie algebra. Let $K$ be a maximal compact subgroup in $G$ and let $\mathfrak{k}$ be the Lie subalgebra in $\mathfrak{g}$ corresponding to $K$. Then one has a Cartan decomposition $\mathfrak{g}=\mathfrak{k}+\mathfrak{p}$, where $\mathfrak{p}$ is a linear subspace of $\mathfrak{g}$. This sum is direct. The Cartan decomposition is characterized by the inclusions

$$
[\mathfrak{k}, \mathfrak{k}] \subset \mathfrak{k}, \quad[\mathfrak{p}, \mathfrak{p}] \subset \mathfrak{k}, \quad[\mathfrak{k}, \mathfrak{p}] \subset \mathfrak{p} .
$$

The formula

$$
B(X, Y)=\operatorname{Tr}((\operatorname{ad} X)(\operatorname{ad} Y)), \quad X, Y \in \mathfrak{g},
$$

determines a symmetric bilinear form on $\mathfrak{g}$, which is called a Killing-Cartan form. Here ad $X$ is the operator on $\mathfrak{g}$ acting as $(\operatorname{ad} X) Y=[X, Y], Y \in \mathfrak{g}$. The fact that a Lie algebra $\mathfrak{g}$ is semisimple means that the bilinear form $B(X, Y)$ is non-degenerate. If $\mathfrak{g}=\mathfrak{k}+\mathfrak{p}$ is a Cartan decomposition of $\mathfrak{g}$, then $B(X, X)<0$ for $X \in \mathfrak{k}$ and $B(X, X)>0$ for $X \in \mathfrak{p}$.

Let $\theta$ be the involutive Cartan automorphism on $\mathfrak{g}$. Then $\theta$ leaves elements of $\mathfrak{k}$ invariant and multiplies elements of $\mathfrak{p}$ by -1 . We introduce on $\mathfrak{g}$ the bilinear form

$$
\langle X, Y\rangle=-B(X, \theta Y)
$$

This form determines a positive scalar product on $\mathfrak{g}$. The algebra $\mathfrak{g}$ with this scalar product turns into a finite dimensional Hilbert space. 
Let $\mathfrak{a}$ be a maximal commutative subalgebra in $\mathfrak{p}$. A dimension of $\mathfrak{a}$ is called a real rank of the Lie algebra $\mathfrak{g}$. We consider the set of operators ad $H, H \in \mathfrak{a}$, acting on the space $\mathfrak{g}$. It is easy to see that

$$
\langle(\operatorname{ad} H) X, Y\rangle=\langle X,(\operatorname{ad} H) Y\rangle, \quad X, Y \in \mathfrak{g},
$$

that is, ad $H$ is a self-adjoint operator on $\mathfrak{g}$. Thus, if $\mathfrak{g}$ is supplied by the scalar product (10.3), then the operators ad $H, H \in \mathfrak{a}$, constitute a commuting collection of self-adjoint operators. For this reason, $\mathfrak{g}$ decomposes into an orthogonal sum of eigenspaces of these operators:

$$
\mathfrak{g}=\mathfrak{g}_{0}+\sum_{\gamma} \mathfrak{g}_{\gamma}
$$

where $\mathfrak{g}_{0}$ is an eigenspace with zero eigenvalue for all operators ad $H, H \in \mathfrak{a}$, and $\mathfrak{g}_{\gamma}$ are eigenspaces with eigenvalues $\gamma(H), H \in \mathfrak{a}$ ( $\gamma$ are linear forms on $\mathfrak{a})$.

It is evident that elements of different eigenspaces $\mathfrak{g}_{\gamma}$ are orthogonal with respect to the scalar product (10.3). Linear forms $\gamma$ on $\mathfrak{a}$ in (10.4) are called restricted roots of the algebra $\mathfrak{g}$ with respect to $\mathfrak{a}$ (or restricted roots of the pair $(\mathfrak{g}, \mathfrak{a})$ ). The subspaces $\mathfrak{g}_{\gamma}$ are called root subspaces.

Restricted roots are split into two sets: a set of positive restricted roots and a set of negative restricted roots. In order to determine a sign of a restricted root we have to fix a basis $H_{1}, H_{2}, \ldots, H_{l}$ of the subalgebra $\mathfrak{a}$. If the first non-zero number in the set $\gamma\left(H_{1}\right), \gamma\left(H_{2}\right), \ldots$, $\gamma\left(H_{l}\right)$ is positive (negative), then the root $\gamma$ is positive (negative). The set $\alpha_{1}, \alpha_{2}, \ldots, \alpha_{l}$ of restricted positive roots is called a system of simple roots if each restricted positive root is a linear combination of $\alpha_{1}, \alpha_{2}, \ldots, \alpha_{l}$ with non-negative integral coefficients.

Restricted roots and root subspaces possess the following properties:

(a) if $\gamma_{1}, \gamma_{2}$ and $\gamma_{1}+\gamma_{2}$ are restricted roots of the pair $(\mathfrak{g}, \mathfrak{a})$, then $\left[\mathfrak{g}_{\gamma_{1}}, \mathfrak{g}_{\gamma_{2}}\right] \subset \mathfrak{g}_{\gamma_{1}+\gamma_{2}} ;$ if $\gamma_{1}+\gamma_{2}$ is not a restricted root, then $\left[\mathfrak{g}_{\gamma_{1}}, \mathfrak{g}_{\gamma_{2}}\right]=0$;

(b) if $\gamma$ is a restricted root, then $-\gamma$ is also a restricted root, that is, to each positive root $\gamma$ there corresponds a negative root $-\gamma$;

(c) root subspaces $\mathfrak{g}_{\gamma}$ may be more than one-dimensional;

(d) only the roots $2 \gamma, \gamma,-\gamma,-2 \gamma$ or the roots $\gamma, \gamma / 2,-\gamma / 2, \gamma$ can be roots multiple to a restricted root $\gamma$; in particular, if a semisimple Lie algebra is of rank 1 , then the subalgebra $\mathfrak{a}$ is one-dimensional and all restricted roots are linearly dependent, that is, in this case there are only two restricted roots $\gamma,-\gamma$ or 4 restricted roots $2 \gamma, \gamma,-\gamma,-2 \gamma$.

A dimension of a root subspace $\mathfrak{g}_{\gamma}$ is called a multiplicity of the restricted root $\gamma$, which is denoted by $m(\gamma)$.

Let $\gamma_{1}, \gamma_{2}, \ldots, \gamma_{n}$ be a set of all positive restricted roots of the pair $(\mathfrak{g}, \mathfrak{a})$. The linear form

$$
\rho=\frac{1}{2} \sum_{i=1}^{n} m\left(\gamma_{i}\right) \gamma_{i}
$$

is called a half-sum of positive restricted roots of the pair $(\mathfrak{g}, \mathfrak{a})$. This form is important in the representation theory of semisimple Lie groups.

Let $\mathfrak{n}=\sum_{\gamma>0} \mathfrak{g}_{\gamma}$, where summation is over positive restricted roots of the pair $(\mathfrak{g}, \mathfrak{a})$. Then $\mathfrak{n}$ is a maximal nilpotent subalgebra in $\mathfrak{g}$ and the Iwasawa decomposition

$$
\mathfrak{g}=\mathfrak{k}+\mathfrak{a}+\mathfrak{n}
$$

holds, where the sum is direct. 
Let $\mathfrak{m}$ be the centralizer of the subalgebra $\mathfrak{a}$ in $\mathfrak{k}$ (that is, the set of all elements of $\mathfrak{k}$ which commute with all elements of $\mathfrak{a}$ ). Then $\mathfrak{m}$ is a subalgebra in $\mathfrak{g}$. Moreover, $\mathfrak{m}$ is a reductive Lie algebra (that is, it is a direct sum of a semisimple subalgebra of $\mathfrak{g}$ and a center of $\mathfrak{m}$ ). It is clear that $\mathfrak{m}$ belongs to the subalgebra $\mathfrak{g}_{0}$ from (10.4). Moreover, the subalgebra $\mathfrak{g}_{0}$ coincides with the sum $\mathfrak{a}+\mathfrak{m}$.

Let $N$ be a closed subgroup in $G$ with a Lie algebra $\mathfrak{n}$ and let $A$ be a subgroup in $G$ with the Lie algebra a. Then $G=A N K$. Moreover, each element $g \in G$ decomposes uniquely as a product $g=h n k, h \in A, n \in N, k \in K$. The mapping $(h, n, k) \rightarrow h n k$ is an analytic diffeomorphism of the manifold $A \times N \times K$ onto $G$. The decomposition of elements of the group $G$ into a product of elements of the subgroups $A, N$ and $K$ is called its Iwasawa decomposition. It is a global analogue of the Iwasawa decomposition $\mathfrak{g}=\mathfrak{k}+\mathfrak{a}+\mathfrak{n}$ of the Lie algebra $\mathfrak{g}$. This decomposition is extensively used in the representation theory.

The Iwasawa decomposition of $G$ can be written in another form. Since $G=G^{-1}=$ $(A N K)^{-1}=K^{-1} N^{-1} A^{-1}=K N A$, then $G=K N A$. Each element $g \in G$ decomposes uniquely as a product $g=k n h, k \in K, n \in N, h \in A$.

Let $M$ be the centralizer of the subgroup $A$ in $K$, that is, $M$ consists of all elements of $K$ commuting with all elements of $A$. Then the subalgebra $\mathfrak{m}$ is a Lie algebra for $M$. The subgroup $M$ may be not connected. It can be represented in the form $M=Z M_{0}$, where $M_{0}$ is a connected component of $M$, containing the unit element, and $Z$ is a finite group. The subgroup $P=A N M=M N A$ of the group $G$ is called a minimal parabolic subgroup of $G$. A subgroup of $G$, containing a minimal parabolic subgroup, is called a parabolic subgroup of $G$. Below we shall consider representations of the group $G$, induced by irreducible representations of a minimal parabolic subgroup.

Let us consider a connection of compact and noncompact Lie groups and a connection of their Lie algebras. Let $G$ and $\mathfrak{g}$ be as above. Since $G$ is a linear group, it has a complexification; let us denote it by $[G]$. Let $[\mathfrak{g}]$ be a complexification of the Lie algebra $\mathfrak{g}$. Let $G_{k}$ be a compact real form of the complex group $[G]$, and let $\mathfrak{g}_{k}$ be a compact real form of the Lie algebra $[\mathfrak{g}]$. If $\mathfrak{g}=\mathfrak{k}+\mathfrak{p}$ is a Cartan decomposition of the Lie algebra $\mathfrak{g}$, then for the Lie algebra $\mathfrak{g}_{k}$ we have the decomposition

$$
\mathfrak{g}_{k}=\mathfrak{k}+\mathrm{ip}, \quad \mathrm{i}=\sqrt{-1},
$$

(see, for example, [16]).There exists also a connection between decompositions of the groups $G$ and $G_{k}$. For $G$, the decomposition $G=K A K$ (the Cartan decomposition) holds. The subgroup $A$ is commutative and noncompact. Moreover, it is a direct product of $l$ copies of the group $\mathbb{R}^{+}$of positive real numbers with the usual multiplication as a group operation. Under complexification of $G$ the subgroup $A \subset G$ turns into the commutative subgroup $[A] \subset[G]$. Then $A_{k}=[A] \cap G_{k}$ is a commutative subgroup in $G_{k}$. Thus, the subgroup $A_{k} \subset G_{k}$ is obtained by analytic continuation of the parameters of the subgroup $A \subset G$ to the corresponding compact domain. To the decomposition $G=K A K$ there corresponds the decomposition $G_{k}=K A_{k} A$ of the compact group $G_{k}$. This fact is used in consideration of finite dimensional representations of semisimple Lie groups. It is known that finite dimensional representations of the groups $G$ and $G_{k}$ are obtained by analytic continuation in group parameters [42]. It follows from the decompositions $G=K A K$ and $G_{k}=K A_{k} K$ that it is enough to make an analytical continuation only for parameters of the subgroups $A$ and $A_{k}$.

More details on the structure of semisimple Lie groups and Lie algebra can be found in $[15,16]$ and [43]. 


\section{Representations of semisimple Lie groups and Lie algebras}

We consider representations of the principal nonunitary series of a noncompact real semisimple Lie group $G$. These representations are constructed by means of irreducible finite dimensional representations of a minimal parabolic subgroup $P=M N A$. Let $\delta$ be a unitary irreducible representation of the compact subgroup $M$ on a finite dimensional Hilbert space $\mathcal{V}$, and let $\lambda$ be a complex linear form on $\mathfrak{a}$. Then the mapping $h \rightarrow \exp (\lambda(\log h)), h \in A$, is a representation of the commutative subgroup $A$. Here log denotes the mapping of the group $A$ onto its Lie algebra $\mathfrak{a}$, which is inverse to the exponential mapping exp $: \mathfrak{a} \rightarrow A$. The correspondence

$$
m n h \rightarrow \exp (\lambda(\log h)) \delta(m), \quad h \in A, \quad n \in N, \quad m \in M,
$$

is an irreducible representation of the group $P=M N A$.

By means of the representations (11.1) of $P$ one constructs (induces) representations of the group $G$. Let us first construct Hilbert spaces on which these representations of $G$ act. Let $f$ be a function from $G$ to the finite dimensional Hilbert space $\mathcal{V}$ satisfying the relation

$$
f(g m n h)=\delta\left(m^{-1}\right) \exp (-\lambda(\log h)) f(g), \quad m \in M, \quad n \in N, \quad h \in A .
$$

These functions are uniquely determined by their values on representatives of cosets in $G / P=$ $G / M N A$. Indeed, each element $g \in G$ is uniquely decomposed into a product $g=x p=x m n h$, $p=m n h \in P=M N A$, where $x$ denotes representatives of $G / P$. Then

$$
f(g)=f(x m n h)=\delta\left(m^{-1}\right) \exp (-\lambda(\log h)) f(x) .
$$

Thus, if we have $f(x)$, then $f(g), g \in G$, are uniquely determined.

Since $G / P=K N A / M N A \sim K / M$, then instead of representatives of $G / P$ we can take representatives of cosets in $K / M$. If we extend the set of the latter representatives to the whole subgroup $K$, then instead of functions $f(g)$ on $G$, satisfying the condition (11.2), we obtain functions on $K$, satisfying the condition

$$
f(k m)=\delta\left(m^{-1}\right) f(k), \quad m \in M .
$$

Now we construct a Hilbert space of functions $f(k)$ on $K$ with values in $\mathcal{V}$, satisfying the condition (11.4) and the conditio

$$
\int_{K}\|f(k)\|_{\mathcal{V}}^{2} d k<\infty
$$

where $d k$ is an invariant measure on $K$ and $\|f(k)\|_{\mathcal{V}}$ is a norm on $\mathcal{V}$. Namely, we define a scalar product in the space of such functions by the formula

$$
\left\langle f_{1}, f_{2}\right\rangle=\int_{K}\left\langle f_{1}(k), f_{2}(k)\right\rangle_{\mathcal{V}} d k
$$

and close this space by means of this scalar product. Note that this scalar product can be introduced both in the space of functions on $G$, satisfying the condition (11.2), and in the space of functions on $K$, satisfying the condition (11.4). In the first case the Hilbert space is denoted by $\mathcal{H}_{\delta \lambda}$ and in the second case by $L_{\delta}^{2}(K, \mathcal{V})$. Now the formula

$$
\pi_{\delta \lambda}\left(g_{0}\right) f(g)=f\left(g_{0}^{-1} g\right), \quad g_{0} \in G,
$$

determines a linear representation of the group $G$ on the space $\mathcal{H}_{\delta \lambda}$ which is denoted by $\pi_{\delta \lambda}$. Going from functions given on $G$ to functions given on $K$ we obtain

$$
\pi_{\delta \lambda}(g) f(k)=\exp (-\lambda(\log h)) f\left(k_{g}\right), \quad g \in G,
$$


where $h \in A$ and $k_{g} \in K$ are determined by the Iwasawa decomposition of the element $g^{-1} k$ :

$$
g^{-1} k=k_{g} n h, \quad n \in N .
$$

Thus, in $L_{\delta}^{2}(K, \mathcal{V})$ the representation $\pi_{\delta \lambda}$ is given by formula (11.7). The representations (11.6) and (11.7), realized on the spaces $\mathcal{H}_{\delta \lambda}$ and $L_{\delta}^{2}(K, \mathcal{V})$, respectively, are in fact different realizations of the same representation. One says that this representation is induced by the representation (11.1) of the subgroup $P$.

If $\delta$ runs over all non-equivalent irreducible unitary representations of the subgroup $M$ and $\lambda$ runs over all complex linear forms on $\mathfrak{a}$, then $\pi_{\delta \lambda}$ constitute the principal nonunitary series of representations of $G$.

The restriction of the representation $\pi_{\delta \lambda}$ to the subgroup $K$ acts on $L_{\delta}^{2}(K, \mathcal{V})$ by the formula

$$
\pi_{\delta \lambda}\left(k_{0}\right) f(k)=f\left(k_{0}^{-1} k\right) .
$$

Since the functions $f$ satisfy the condition $f(k m)=\delta\left(m^{-1}\right) f(k), m \in M$, the space $L_{\delta}^{2}(K, \mathcal{V})$ decomposes into orthogonal sum of linear finite dimensional subspaces, on which irreducible unitary representations (we denote them by $\nu$ ) of the subgroup $K$ are realized. Moreover, each such representation $\nu$ is contained in the decomposition of the representation $\pi_{\delta \lambda} \downarrow_{K}$ of $K$ with multiplicity $b_{\delta}^{\nu}$, where $b_{\delta}^{\nu}$ is the multiplicity of the representation $\nu$ of $M$ in the representation $\delta$ of $K$.

The representation $\pi_{\delta \lambda}$ of the group $G$ determines the corresponding representation of the Lie algebra $\mathfrak{g}$, which is denotes also by $\pi_{\delta \lambda}$. To noncompact elements of the Lie algebra $\mathfrak{g}$ there correspond unbounded operators of this representation. For this reason, these operators are not defined on the whole Hilbert space $L_{\delta}^{2}(K, \mathcal{V})$. However, each of these operators is determined on differentiable functions from $L_{\delta}^{2}(K, \mathcal{V})$. Moreover, on the set of infinitely differentiable functions of $L_{\delta}^{2}(K, \mathcal{V})$ an action of products of such operators is determined. In particular, an action of Casimir operators is determined on such functions. Note that the linear space of infinitely differentiable functions of $L_{\delta}^{2}(K, \mathcal{V})$ is dense in this Hilbert space.

\section{Hyperboloids and cones for semisimple noncompact Lie groups and coordinate systems on them}

\subsection{Hyperboloids and cones}

Let us construct analogues of upper sheet of the hyperboloid $H_{+}^{4}$ of the two-sheeted hyperboloid and the upper sheet $C_{+}^{4}$ of the cone constructed in the previous sections. The motion group of $H_{+}^{4}$ and of $C_{+}^{4}$ is the de Sitter group $S O_{0}(1,4)$. The hyperboloid $H_{+}^{4}$ is identified with the homogeneous space $S O_{0}(1,4) / S O(4)$, where $S O(4)$ is a maximal compact subgroup in $S O_{0}(1,4)$, and the cone $C_{+}^{4}$ with the homogeneous space $S O_{0}(1,4) / I S O(3)$, where $I S O(3)$ is the subgroup $M N$ (see the previous section) for $S O_{0}(1,4)$. Taking into account these facts, we define a hyperboloid and a cone for an arbitrary connected linear semisimple noncompact Lie group $G$ as follows. An analogue of the upper sheet $H_{+}^{4}$ of the two-sheeted hyperboloid is the homogeneous space $G / K$, where $K$ is a maximal compact subgroup of $G$. The space $G / K$ is a noncompact Riemannian symmetric space (see [16]). In order to have an analogy with the case of the group $S O_{0}(1,4)$, we call this space a hyperboloid. A cone (an analogue of the upper sheet $C_{+}^{4}$ of the cone) with a motion group $G$ is the homogeneous space $G / M N$, where $N$ is a maximal nilpotent subgroup (see the previous section) and $M$ is a subgroup of $K$, which is a centralizer of the subgroup $A=\exp \mathfrak{a}$ in $K$. A list of classical simple noncompact real Lie groups $G$ and their subgroups $K$ and $M$ is given in Table 1 . 
Table 1

\begin{tabular}{|c|c|c|c|}
\hline$G$ & $G_{k}$ & $K$ & $M$ \\
\hline$S L(n, \mathbb{C})$ & $S U(n) \times S U(n)$ & $S U(n)$ & $D$ \\
\hline$S O(n, \mathbb{C})$ & $S O(n) \times S O(n)$ & $S O(n)$ & $D$ \\
\hline$S p(n, \mathbb{C})$ & $S p(n) \times S p(n)$ & $S p(n)$ & $\left(Z_{2}\right)_{n-1}$ \\
\hline$S L(n, \mathbb{R})$ & $S U(n)$ & $S O(n)$ & $\left(Z_{2}\right)_{n-1}$ \\
\hline$S U^{*}(2 n)$ & $S U(2 n)$ & $S p(n)$ & $S(U(p-q) \times U(1) \times \cdots \times U(1))$ \\
\hline$S U(p, q)$ & $S U(p+q)$ & $S(U(p) \times U(q))$ & $S(1) \times \cdots \times U(1)$ \\
\hline$U(p, q)$ & $U(p+q)$ & $U(p) \times U(q)$ & $U(p-q) \times U(1) \times \cdots\left(Z_{2}\right)_{q}$ \\
\hline$S O_{0}(p, q)$ & $S O(p+q)$ & $S O(p) \times S O(q)$ & $S O(p-q) \times(2) \times \cdots \times S U(2)$ \\
\hline$S O^{*}(2 n)$ & $S O(2 n)$ & $U(n)$ & $S U(2) \times \cdots(2) \times U(1)$ \\
& & & if $n=2 k$ \\
& & & if $n=2 k+1$ \\
\hline$S p(n, \mathbb{R})$ & $S p(n)$ & $U(n)$ & $\left(Z_{2}\right)_{n-1}$ \\
\hline$S p(p, q)$ & $S p(p+q)$ & $S p(p) \times S p(q)$ & $S p(p-q) \times S p(1) \times \cdots \times S p(1)$ \\
\hline
\end{tabular}

Complex groups $S L(n, \mathbb{C}), S O(n, \mathbb{C})$ and $S p(n, \mathbb{C})$ in Table 1 are understood as real groups with double number of real parameters. The subgroups $D$ in these groups coincide with maximal torus in $K . Z_{2}$ denotes a group consisting of two elements and $\left(Z_{2}\right)_{m}$ is a direct product of $m$ copies of the group $Z_{2}$.

The nilpotent subgroup $N$ of $G$ is obtained by exponential mapping from the nilpotent subalgebra $\mathfrak{n}$ of the Lie algebra $\mathfrak{g}$. The subalgebra $\mathfrak{n}$ is constructed by means of the system of restricted roots of the pair $(\mathfrak{g}, \mathfrak{a})$. In [44], examples of construction of subalgebras $\mathfrak{n}$ are given. The subgroup $N$ for the group $S O_{0}(1, n)$ is constructed in [3], Chapter 9. The subgroup $N$ of the group $S U(1, n)$ is given in [44].

\subsection{Cartan decomposition and $S$-coordinate system on $G / K$}

The Cartan decomposition of the group $G$ is given by the formula $G=K A K$. This means that each element $g \in G$ can be represented in the form of a product $k h k^{\prime}, k, k^{\prime} \in K, h \in A$. However the decomposition $g=k h k^{\prime}$ is not unique since $k m h k^{\prime}=k h m k^{\prime}, m \in M$. Therefore, taking a decomposition of elements of $K$ in the form $k=\tilde{k} m, m \in M$, where $\tilde{k}$ are representatives of cosets of the homogeneous space $K / M$, we obtain from the Cartan decomposition $g=k h k^{\prime}$ of $g \in G$ the decomposition

$$
g=\tilde{k} h k \quad \text { or } \quad g=k h \tilde{k} .
$$

The set of elements $\tilde{k}$ of $K$, parametrizing the space $K / M$, is denoted by $(K / M)$. An ambiguousness of the decomposition (12.1) is determined by the relation

$$
\tilde{k} h m k=\tilde{k} m h k, \quad m \in M^{*} / M \equiv W,
$$

where $M^{*}$ is a normalizer of the subgroup $A$ in $K$. The quotient group $W$ is finite and is called a Weyl group of the pair $(\mathfrak{g}, \mathfrak{a})$. Thus, the decomposition (12.1) will be unique almost for each $g \in G$ if $h$ is taken from the part $\overline{A^{+}}$of the subgroup $A$, where $\overline{A^{+}}$is a closure of the set $A^{+}$. The set $\overline{A^{+}}$is characterized by the property that there are no two elements $h$ and $h^{\prime}$ in $\overline{A^{+}}$ such that $h=m h^{\prime} m^{-1}$ for some $m \in M^{*}$. The set $\overline{A^{+}}$can be constructed in the following way. The set $A^{+}$is the set of all elements $h=\exp H, H \in \mathfrak{a}$, satisfying the conditions $\alpha_{i}(H)>0$, $i=1,2, \ldots, l$, where $\alpha_{i}, i=1,2, \ldots, l$, are simple restricted roots of the pair $(\mathfrak{g}, \mathfrak{a})$. The set $\overline{A^{+}}$ coincides with the closure of the set $A^{+}$in $A$. Thus, starting from the decomposition

$$
G=(K / M) \overline{A^{+}} K,
$$


we obtain the decomposition $g=\tilde{k} h k, \tilde{k} \in(K / M), h \in \overline{A^{+}}, k \in K$, of elements of the group $G$. According to the formula (12.2), there exists a set $(G / K)$ of representative of cosets of $G / K$,

$$
(G / K)=(K / M) \overline{A^{+}},
$$

such that to each element of $(G / K)$ there corresponds only one coset in $G / K$ and vise versa.

If $l$ is a real rank of the group $G$, then for elements $h \in A$ we have

$$
h=\exp H=\exp \left(t_{1} H_{1}+t_{2} H_{2}+\cdots+t_{l} H_{l}\right)=\prod_{i=1}^{l} \exp t_{i} H_{i},
$$

where $H_{1}, H_{2}, \ldots, H_{l}$ is a basis of the subalgebra a. The part $\overline{A^{+}}$of the subgroup $A$ is characterized by the condition that the parameters $t_{1}, t_{2}, \ldots, t_{l}$ of elements $h=\exp H \in \overline{A^{+}}$satisfy the conditions $\alpha_{i}(H) \equiv \alpha_{i}\left(t_{1} H_{1}+t_{2} H_{2}+\cdots+t_{l} H_{l}\right) \geq 0, i=1,2, \ldots, l$. It follows from (12.3) that for obtaining a parametrization of the space $G / K$ we have to determine a parametrization of the "sphere" $K / M$. It is clear that the space $K / M$ is compact. The numbers $t_{1}, t_{2}, \ldots, t_{l}$ together with parameters, determining $K / M$, parametrize the set $(G / K)$.

A parametrization of the space $K / M$ is not unique. It can be received as follows. Let $G_{k}^{\prime}$ and $G^{\prime}$ be compact and noncompact connected semisimple real Lie groups with the same complexification $[G]$. The pairs $\left(G^{\prime}, G_{k}^{\prime}\right)$, corresponding to simple Lie groups $G^{\prime}$, are given in Table 1. Let $K^{\prime}$ be a maximal compact subgroup in $G^{\prime}$ and let $G^{\prime}=K^{\prime} A^{\prime} K^{\prime}$ be a Cartan decomposition of the group $G^{\prime}$. The group $G_{k}^{\prime}$ is obtained from the group $G^{\prime}$ by an analytic continuation (in the framework of the complexification $\left[G^{\prime}\right]$ of $G^{\prime}$ ) of noncompact parameters of the group $G^{\prime}$ to the corresponding compact parameters of the group $G_{k}^{\prime}$. It follows from the Cartan decomposition $G^{\prime}=K^{\prime} A^{\prime} K^{\prime}$ that we have to make only an analytical continuation of parameters of the subgroup $A^{\prime}$. As a result of this continuation, we obtain the decomposition $G_{k}^{\prime}=K^{\prime} A_{k}^{\prime} K^{\prime}$ of the group $G_{k}^{\prime}$ with the commutative subgroup (torus) $A_{k}^{\prime}$. If elements of the subgroup $A^{\prime}$ are of the form (12.4), then elements of the subgroup $A_{k}^{\prime}$ are of the form

$$
\prod_{i=1}^{l} \exp \varphi_{i} H_{i}, \quad 0 \leqslant \varphi_{j}<2 \pi .
$$

The decomposition $G_{k}^{\prime}=K^{\prime} A_{k}^{\prime} K^{\prime}$ is not unique. The reason is that elements of the subgroup $M^{\prime} \subset K^{\prime}$ permute with elements of $A_{k}^{\prime}$. Therefore, as in the case of noncompact simple real Lie groups, we have the decomposition $G_{k}^{\prime}=\left(K^{\prime} / M^{\prime}\right) A_{k}^{\prime} K^{\prime}$. This decomposition is not unique, since elements from $M^{\prime *} / M^{\prime} \equiv W^{\prime}$, as well as elements from the set $J=K^{\prime} \cap A_{k}^{\prime}$, permute with $A_{k}^{\prime}$ (for a noncompact group $G^{\prime}$ the set $J=K^{\prime} \cap A^{\prime}$ consists of one (unit) element). Restricting (in an appropriate manner) values of the parameters $\varphi_{1}, \varphi_{2}, \ldots, \varphi_{l}$ in (12.5), we obtain the decomposition $G_{k}^{\prime}=\left(K^{\prime} / M^{\prime}\right) \overline{A_{k}^{\prime}} K^{\prime}$. This decomposition leads to the decomposition of the set $\left(G_{k}^{\prime} / K^{\prime}\right)$ :

$$
\left(G_{k}^{\prime} / K^{\prime}\right)=\left(K^{\prime} / M^{\prime}\right) \overline{A_{k}^{\prime+}} .
$$

Now we set here $G_{k}^{\prime}=K$ and $K^{\prime}=M$ and find

$$
(K / M)=\left(M / M^{\prime}\right) \overline{A_{k}+},
$$

where $A_{k}$ is a commutative subgroup (torus) in $K$ and $M^{\prime}$ is a subgroup in $K$ and in $M$. Note that the subgroup $M$ can be nonconnected. Then it is a product of a connected subgroup $M_{0}$ with a finite subgroup $Z$. In this case the decomposition (12.6) must be applied to the space 
$K / M_{0}$ (not to the space $K / M$ ). Then for transition from $K / M_{0}$ to $K / M$ we have to perform an identification of the corresponding values of parameters.

The relation (12.7) leads a parametrization of the space $K / M$ to a parametrization of the space $M / M^{\prime}$ such that $\operatorname{dim} M / M^{\prime}<\operatorname{dim} K / M$. We may apply decomposition (12.6) to the set $M / M^{\prime}$ and reduce its parametrization to a parametrization of a space which has smaller dimension than that of $M / M^{\prime}$. Continuing this procedure, after a finite number of steps we obtain a parametrization of the whole space $K / M$, and therefore of the space $G / K$. A parametrization, obtained in this way, corresponds to the chain of subgroups $G \supset K \supset M \supset M^{\prime} \supset \cdots$. Such coordinate system is an analogue of the spherical system ( $S$-system) of coordinates for the hyperboloid $H_{4}^{+}$. For this reason, we call such set of coordinates on $G / K$ a spherical coordinate system or an $S$-system of coordinates.

Thus, a parametrization of the space $G / K$ was obtained by using the Cartan decompositions of the semisimple Lie group $G$ and of the corresponding compact Lie groups $G_{k}^{\prime}$. By using these decompositions we can receive a $G$-invariant measure on $G / K$, expressed in terms of the corresponding parameters. If $f$ is a continuous function on $G$ with compact support and $g=k_{1}(\exp H) k_{2}, k_{1}, k_{2} \in K, h=\exp H \in A$, then the following formula holds (see [16]):

$$
\int_{G} f(g) d g=c \frac{1}{|W|} \int_{\mathfrak{a}}\left|\prod_{\alpha>0} \sinh \alpha(H)\right| d H \int_{K} \int_{K} f\left(k_{1}(\exp H) k_{2}\right) d k_{1} d k_{2},
$$

where $d g$ and $d k$ are invariant measures on $G$ and $K$, respectively; the product is over the set of positive restricted roots of the pair $(\mathfrak{g}, \mathfrak{a})$ (and each root $\alpha$ appears in the product the number of times equal to multiplicity of this root), $d H$ is the Lebesgue measure on the space $\mathfrak{a},|W|$ is an order of the Weyl group $W$ of the pair $(\mathfrak{g}, \mathfrak{a})$, and $c$ is a constant, depending on a normalization of measures on $G, \mathfrak{a}$ and $K$.

The formula

$$
\int_{G} f(g) d g=\int_{G / K}\left(\int_{K} f(x k) d k\right) d x
$$

determines an invariant measure $d x$ on $G / K$. Applying this formula to the relation (12.8) we obtain

$$
\int_{G / K} f(x) d x=c_{1} \frac{1}{|W|} \int_{\mathfrak{a}}\left|\prod_{\alpha>0} \sinh \alpha(H)\right| d H \int_{(K / M)} f(y(\exp H)) d y,
$$

where $d y$ is an invariant (with respect to $K$ ) measure on $K / M$. In order to obtain integration over $\overline{A^{+}}$instead of integration over $A=\exp \mathfrak{a}$, it is necessary to restrict the integral over $\mathfrak{a}$ to an integral over $\mathfrak{a}^{+}$. The set $\mathfrak{a}^{+}$consists of elements $H \in \mathfrak{a}$ such that $\alpha_{i}(H)>0$ for any restricted simple root $\alpha_{i}$ of the pair $(\mathfrak{g}, \mathfrak{a})$. The complete algebra $\mathfrak{a}$ is obtained from $\mathfrak{a}^{+}$by action by elements of the Weyl group $W$ (with a subsequent closure). (Note that for any $w_{1}, w_{2} \in W$ the sets $w_{1} \mathfrak{a}^{+}$and $w_{2} \mathfrak{a}^{+}$do not intersect if $w_{1} \neq w_{2}$.) Then

$$
\int_{G / K} f(x) d x=c_{1} \int_{\mathfrak{a}^{+}}\left|\prod_{\alpha>0} \sinh \alpha(H)\right| d H \int_{(K / M)} f(y(\exp H)) d y .
$$

This relation reduces a $G$-invariant measure on $G / K$ to a $K$-invariant measure on $K / M$. In order to obtain the latter measure we use the integral relation for $G_{k}^{\prime}$, which is an analogue of the formula (12.8) (see [16]):

$$
\int_{G_{k}^{\prime}} f(g) d g=c \int_{A_{k}^{\prime}}|D(h)| d h \int_{K^{\prime}} \int_{K^{\prime}} f\left(k_{1} h k_{2}\right) d k_{1} d k_{2} .
$$


The function $D(h) \equiv D(\exp \mathrm{i} H), H \in \mathfrak{a}, h \in A_{k}^{\prime}$, is determined by the relation

$$
D(\operatorname{expi} H)=\prod_{\alpha>0} \sin \alpha(H) .
$$

The relation (12.11) can be easily reduced to the relation similar to (12.10). This gives a possibility to determine a $K$-invariant measure on $K / M$ if we set $G_{K}^{\prime}=K$ and $K^{\prime}=M$.

Remark. The formulas (12.10) and (12.11) contain products over restricted positive roots of the pair $(\mathfrak{g}, \mathfrak{a})$. Systems of simple restricted roots (including their multiplicities) of pairs $(\mathfrak{g}, \mathfrak{a})$ for all simple noncompact Lie algebras $\mathfrak{g}$ are given in [15] (see also Table 3 in [44]). A system of simple roots determines uniquely a system of the corresponding positive roots.

\subsection{Iwasawa decomposition and $T$-coordinate system on $G / K$}

Let $G=N A K$ be an Iwasawa decomposition of the group $G$. It gives a possibility to parametrize the space $G / K$ by means of elements of the subgroup $N A$. The formula (12.4) gives a parametrization of the subgroup $A$ by numbers $t_{1}, t_{2}, \ldots, t_{l}$. In order to obtain a parametrization of the subgroup $N$, it is necessary to represent $N$ in the form of a product of the one-parameter subgroups exp $s_{\alpha} X_{\alpha}$ corresponding to root elements $X_{\alpha}$ of the algebra $\mathfrak{g}$ with positive restricted roots $\alpha$. In an analogy with the group $S O_{0}(1,4)$, this parametrization of $G$ is called a translational system of coordinates on $G / K$, which is also called a T-system of coordinates. Note that for a generic semisimple Lie group $G$ the subgroup $N$ is not commutative. The subgroup $N=\exp \mathfrak{n}$ is commutative if and only if the corresponding Lie algebra $\mathfrak{n}$ is commutative. A commutativity of the algebra $\mathfrak{n}$ is determined by means of a root system of the pair $(\mathfrak{g}, \mathfrak{a})$. If along with roots $\alpha$ and $\beta$ there exists a root $\alpha+\beta$ (or along with a root $\alpha$ there exists a root $2 \alpha$ ), then for the corresponding root subspaces we have $\left[\mathfrak{g}_{\alpha}, \mathfrak{g}_{\beta}\right] \subset \mathfrak{g}_{\alpha+\beta}$. This leads to a violation of commutativity in $\mathfrak{n}$ and, therefore, in $N$. Analyzing systems of simple restricted roots of simple real Lie algebras (see, for example, [44]), we conclude that a subgroup $N$ is commutative only for the groups $S O_{0}(1, n)$.

Using the Iwasawa decomposition $G=N A K$, it is possible to find a $G$-invariant measure on $G / K$, expressed in the $T$-coordinate system. If $f$ is a continuous function with a compact support and $g=n h k, n \in N, h \in A, k \in K$, then (see, for example, [16])

$$
\int_{G} f(g) d g=\int_{K} \int_{A} \int_{N} f(n h k) e^{2 \rho(\log h)} d n d h d k,
$$

where $d g, d n, d h$ and $d k$ are invariant measures on $G, N, A$ and $K$, respectively, and $\rho=\frac{1}{2} \sum_{\alpha>0} \alpha$. Therefore, if an element $x \in G / K$ is represented by an element $n h \in N A$, then

$$
\int_{G / K} f(x) d x=\int_{A} \int_{N} f(n h) e^{2 \rho(\log h)} d n d h,
$$

where $d x$ is a $G$-invariant measure on $G / K$.

\subsection{Iwasawa decomposition and $O$-coordinate system on $G / K$}

Using the Iwasawa decomposition $G=N A K$ of the group $G$, we represent $G$ in the form

$$
G=(N M) A K
$$

where $N M$ is a closed subgroup of $G$. Since $M N M^{-1} \subset N$, then the subgroup $N M$ is a semidirect product of the subgroup $M$ and the normal subgroup $N$. The most interesting case is when 
$N M$ is a semidirect product of the compact subgroup $M$ and the commutative subgroup $N$. As we know this is the case when $G=S O_{0}(1, n)$. For this group the subgroup $N M$ is isomorphic to the group $I S O(n-1)$ (see, for example, [3], Chapter 9).

The decomposition (12.13) is not unique. Indeed, elements of the subgroup $A$ commute with elements of $M$. In order to obtain non-ambiguous decomposition we have to take the space $N M / M$ instead of the space $N M$. Then for $(G / K)$ we have the decomposition

$$
(G / K)=(N M / M) A,
$$

where $(N M / M)$ is the set of representatives of cosets of the quotient space $N M / M$. We take a parametrization of the subgroup $A$ by the numbers $t_{1}, t_{2}, \ldots, t_{l}$. Choosing a parametrization of the quotient space $N M / M$ we obtain, according to the formula (12.4), a parametrization of the space $G / K$. This parametrization is called orispherical. It is also called an $O$-coordinate system on $G / K$. Note that one of possibilities for parametrization of $(N M / M)$ is given by the $T$-system of coordinates.

If we choose (according to a parametrization of the space $N M / M$ ) representatives $y \in N M$ of cosets of $N M / M$ and set $x=y h \in(G / K)$, then we have the integral relation

$$
\int_{G / K} f(x) d x=\int_{N M / M} d y \int_{A} f(y h) e^{2 \rho(\log h)} d h,
$$

where $d h$ is an invariant measure on $A, d x$ is a $G$-invariant measure on $G / K$, and $d y$ is a $N M$ invariant measure on $N M / M$.

\subsection{Generalized Cartan decomposition}

Let $G$ be a semisimple noncompact connected linear real Lie group and let $\mathfrak{g}$ be its Lie algebra. There exists an involutive Cartan automorphism $\theta$ on $\mathfrak{g}$ such that $\mathfrak{g}$ decomposes into a direct sum of eigenspaces of $\theta$ as $\mathfrak{g}=\mathfrak{k}+\mathfrak{p}$ (the Cartan decomposition), where $\mathfrak{k}$ corresponds to eigenvalue +1 and $\mathfrak{p}$ corresponds to eigenvalue -1 . Under transition from $\mathfrak{g}$ to $G$, to the automorphism $\theta$ of $\mathfrak{g}$ there corresponds the automorphism $\Theta$ of $G$. The set of points of $G$, which are invariant with respect to $\Theta$, coincides with the maximal compact subgroup $K$ of $G$. The homogeneous space $G / K$ is a Riemannian symmetric space, which is called a hyperboloid. Thus, we associate a Riemannian symmetric space with the involutive Cartan automorphism $\theta$ of $\mathfrak{g}$.

However, the Lie algebra $\mathfrak{g}$ can have other involutive automorphisms, that is, automorphisms with noncompact stationary subgroup. Let $\tau$ be such an automorphism of $\mathfrak{g}$. Then $\mathfrak{g}$ decomposes into a direct sum of eigenspaces of $\tau$ with eigenvalues \pm 1 (since $\tau^{2}=1$, other eigenvalues cannot exist). Let this decomposition be of the form

$$
\mathfrak{g}=\mathfrak{b}+\mathfrak{q},
$$

where $\mathfrak{b}$ belongs to eigenvalue +1 and $\mathfrak{q}$ to eigenvalue -1 . Since $\tau$ is an automorphism, then the eigenvalues show that

$$
[\mathfrak{b}, \mathfrak{b}] \subset \mathfrak{b}
$$

that is, $\mathfrak{b}$ is a subalgebra of $\mathfrak{g}$. Let $\mathcal{T}$ be an automorphism of the group $G$, corresponding to the automorphism $\tau$ of $\mathfrak{g}$, and let $G_{\tau}$ be a closed subgroup of $G$ consisting of all points of $G$ invariant under $\mathcal{T}$. A connected component of the unit element in $G_{\tau}$ will be denoted by $G_{\tau}^{0}$. If $H$ is a subgroup of $G$ such that $G_{\tau}^{0} \subset H \subset G_{\tau}$, then the homogeneous space $G / H$ is called symmetric. Since the subgroup $H$ is not compact, a quotient space $G / H$ is called pseudo-Riemannian or affine symmetric space. The group $G$ is a motion group of the pseudo-Riemannian symmetric space $G / H$. A classification of pseudo-Riemannian symmetric spaces is given in [45]. 
With a pseudo-Riemannian symmetric space with the transitive motion group $G$ a generalized Cartan decomposition of $G$ is connected. For simplicity, we shall consider only those pseudoRiemannian symmetric spaces for which the automorphism $\tau$ is determined by a signature of the set of restricted roots of the pair $(\mathfrak{g}, \mathfrak{a})$ (see [46]).

Let $\Sigma$ be a set of all restricted roots of the pair $(\mathfrak{g}, \mathfrak{a})$. A mapping $\varepsilon$ of roots of $\Sigma$ to the set $\{+1,-1\}$ is called a signature of $\Sigma$ if

(a) $\varepsilon(\alpha)=\varepsilon(-\alpha), \alpha \in \Sigma$;

(b) if $\alpha, \beta, \alpha+\beta$ are restricted roots, then $\varepsilon(\alpha+\beta)=\varepsilon(\alpha) \varepsilon(\beta)$.

The Lie algebra $\mathfrak{g}$ can be represented in the form

$$
\mathfrak{g}=\mathfrak{m}+\mathfrak{a}+\sum_{\alpha \in \Sigma} \mathfrak{g}_{\alpha}
$$

where $\mathfrak{g}_{\alpha}$ is a root subspace corresponding to a root $\alpha \in \Sigma$. Using a signature $\varepsilon$, we give an automorphism $\theta_{\varepsilon}$ on $\mathfrak{g}$ such that

(a) $\theta_{\varepsilon}(X)=\varepsilon(\alpha) \theta(X), X \in \mathfrak{g}_{\alpha}$, where $\theta$ is the involutive Cartan automorphism, introduced above;

(b) $\theta_{\varepsilon}(X)=X$ for $X \in \mathfrak{m}+\mathfrak{a}$.

It is easy to show that $\theta_{\varepsilon}$ is an involutive automorphism of $\mathfrak{g}$ such that $\theta_{\varepsilon} \neq \theta$ for a nontrivial signature $\varepsilon$ on $\Sigma$.

We write down the decomposition (12.16) for the automorphism $\theta_{\varepsilon}$ in the form

$$
\mathfrak{g}=\mathfrak{t}_{\varepsilon}+\mathfrak{p}_{\varepsilon},
$$

where $\mathfrak{t}_{\varepsilon}$ is a Lie subalgebra (noncompact) in $\mathfrak{g}$. We have $\mathfrak{m} \subset \mathfrak{t}_{\varepsilon}$ and $\mathfrak{a} \subset \mathfrak{p}_{\varepsilon}$.

If the automorphism $\theta_{\varepsilon}$ can be continued to an automorphism of $G$, we denote this automorphism of $G$ by $\Theta_{\varepsilon}$. Let $\left(K_{\varepsilon}\right)_{0}$ be an analytical subgroup of $G$ with the Lie algebra $\mathfrak{t}_{\varepsilon}$. We set $K_{\varepsilon}=\left(K_{\varepsilon}\right)_{0} M$. Since the connected component $M_{0}$ of the unit element in the subgroup $M$ is contained in $\left(K_{\varepsilon}\right)_{0}$ ( since $\mathfrak{m} \subset \mathfrak{k}_{\varepsilon}$ ), then $K_{\varepsilon}=\left(K_{\varepsilon}\right)_{0} Z$, where $Z$ is a discrete subgroup such that $M=M_{0} Z$. The subgroups $\left(K_{\varepsilon}\right)_{0}$ and $K_{\varepsilon}$ have the following properties (see [46]:

(a) for each $m \in M$ we have $m\left(K_{\varepsilon}\right)_{0} m^{-1} \subset\left(K_{\varepsilon}\right)_{0}$;

(b) $K_{\varepsilon}$ is a closed subgroup of $G$ and $\Theta_{\varepsilon} k=k$ for each $k \in K_{\varepsilon}$.

Let us give a classification of pairs $\left(G, K_{\varepsilon}\right)$ for simple Lie groups $G$. A pair $\left(G, K_{\varepsilon}\right)$ is determined by the corresponding pair $\left(\mathfrak{g}, \mathfrak{k}_{\varepsilon}\right)$ for the Lie algebra $\mathfrak{g}$. In order to classify pairs $\left(\mathfrak{g}, \mathfrak{k}_{\varepsilon}\right)$ with a simple Lie algebra $\mathfrak{g}$, we consider signatures of the corresponding root system $\Sigma$. These signatures can be easily described as follows. Let $\alpha_{1}, \alpha_{2}, \cdots, \alpha_{l}$ be simple restricted roots of the pair $(\mathfrak{g}, \mathfrak{a})$. Each restricted root $\alpha \in \Sigma$ can be represented uniquely in the form

$$
\alpha=\sum_{i=1}^{l} m_{i} \alpha_{i} .
$$

Let $\varepsilon_{j}(j=1,2, \ldots, l)$ be a signature of $\Sigma$ such that $\varepsilon_{j}(\alpha)=(-1)^{m_{j}}$, where $m_{j}$ is determined by the decomposition (12.18). Then any signature $\varepsilon$ of the root system $\Sigma$ reduces to some signature $\varepsilon_{j}$, namely, for any signature $\varepsilon$ there exists an element $w$ of the Weyl group $W$ and 
an integer $j(1 \leqslant j \leqslant l)$ such that for each $\alpha \in \Sigma$ we have $\varepsilon(\alpha)=\varepsilon_{j}(w \alpha)$ (see [47]). Thus, any signature $\varepsilon$ can be transformed to some signature $\varepsilon_{j}$.

Let us describe signatures $\varepsilon_{j}$ and the corresponding systems of roots $\Sigma_{\varepsilon}$, where $\Sigma_{\varepsilon}$ is a set of roots $\alpha$ from $\Sigma$ for which $\varepsilon(\alpha)=1$.

Let a root system $\Sigma$ coincide with the root system $A_{l}, l \geqslant 1$. We have signatures

$$
\varepsilon_{j}, \quad 2 j<l+1 .
$$

The corresponding $\Sigma_{\varepsilon_{j}}$ coincide respectively with the root systems

$$
A_{l-j}+A_{j-1} \text {. }
$$

Let a root system $\Sigma$ coincide with the root system $B_{l}, l \geqslant 2$. We have signatures

$$
\varepsilon_{j}, \quad j \leqslant l .
$$

The corresponding $\Sigma_{\varepsilon_{j}}$ coincide respectively with the root systems

$$
B_{l-j}+D_{j}
$$

Let $\Sigma$ coincide with the root system $B C_{l}, l \geqslant 1$. We have signatures

$$
\varepsilon_{j}, \quad j \leqslant l .
$$

The corresponding $\Sigma_{\varepsilon_{j}}$ coincide respectively with the root systems

$$
B C_{l-j}+C_{j}
$$

Let $\Sigma$ coincide with the root system $C_{l}, l \geqslant 3$. We have signatures

$$
\varepsilon_{j}, \quad 2 j \leqslant l, \quad \varepsilon_{l} .
$$

The corresponding $\Sigma_{\varepsilon_{j}}$ coincide respectively with the root systems

$$
C_{l-j}+C_{j} \quad \text { if } \quad 2 j \leqslant l
$$

and

$A_{l} \quad$ for $\quad \varepsilon_{l}$.

Let $\Sigma$ coincide with the root system $D_{l}, l \geqslant 4$. Then we have signatures

$$
\varepsilon_{j}, \quad 2 j \leqslant l, \quad \varepsilon_{l-1}, \quad \varepsilon_{l} .
$$

The corresponding $\Sigma_{\varepsilon_{j}}$ coincide respectively with the root systems

$$
D_{l-j}+D_{j} \quad \text { if } \quad 2 j \leqslant l
$$

and

$$
A_{l} \quad \text { for } \quad \varepsilon_{l-1} \quad \text { and } \quad \varepsilon_{l} \text {. }
$$

Let $\Sigma$ coincide with the root system $E_{6}$. We have signatures

$\varepsilon_{1} \quad$ and $\quad \varepsilon_{2}$. 
Then the corresponding $\Sigma_{\varepsilon_{j}}$ coincide respectively with the root systems

$$
D_{5} \quad \text { for } \quad \varepsilon_{1}
$$

and

$$
A_{1}+A_{5} \quad \text { for } \quad \varepsilon_{2} .
$$

Let a root system $\Sigma$ coincide with the root system $E_{7}$. We have signatures

$$
\varepsilon_{j}, \quad j=1,2,7 .
$$

The corresponding $\Sigma_{\varepsilon_{j}}$ coincide respectively with the root systems

$$
\begin{array}{llll}
A_{1}+D_{6} & \text { for } & \varepsilon_{1}, \\
A_{7} & \text { for } & \varepsilon_{2}, & \\
E_{6} & \text { for } & \varepsilon_{7} . &
\end{array}
$$

Let $\Sigma$ coincide with the root system $E_{8}$. We have signatures

$$
\varepsilon_{j}, \quad j=1,8 .
$$

The corresponding $\Sigma_{\varepsilon_{j}}$ coincide respectively with the root systems

$$
D_{8} \quad \text { for } \quad \varepsilon_{1}
$$

and

$$
A_{1}+E_{7} \quad \text { for } \quad \varepsilon_{8} \text {. }
$$

Let $\Sigma$ coincide with the root system $F_{4}$. We have signatures

$$
\varepsilon_{j}, \quad j=1,4 .
$$

The corresponding $\Sigma_{\varepsilon_{j}}$ coincide respectively with the root system

$$
A_{1}+C_{3} \quad \text { for } \quad \varepsilon_{1}
$$

and with root system

$$
B_{4} \quad \text { for } \quad \varepsilon_{4} \text {. }
$$

Let a root system $\Sigma$ coincide with the root system $G_{2}$. Then we have only one signature $\varepsilon=1$. The corresponding $\Sigma_{\varepsilon_{j}}$ is the root system of

$$
A_{1}+A_{1} \text {. }
$$

For a fixed $\Sigma$, there can be several corresponding pairs $\left(\mathfrak{g}, \mathfrak{k}_{\varepsilon}\right)$. Let us give a list of possible subalgebras $\mathfrak{k}_{\varepsilon}$ for classical simple real Lie algebras:

$$
\begin{array}{lll}
\mathfrak{g}=\operatorname{sl}(l+1, \mathbb{C}): & \mathfrak{k}_{\varepsilon}=\operatorname{su}(l-j+1, j), \quad 0 \leqslant 2 j \leqslant l ; \\
\mathfrak{g}=\operatorname{sl}(l+1, \mathbb{R}): & \mathfrak{k}_{\varepsilon}=\operatorname{so}(l-j+1, j), \quad 0 \leqslant 2 j \leqslant l ; \\
\mathfrak{g}=\operatorname{su}^{*}(2 l+2): & \mathfrak{k}_{\varepsilon}=\operatorname{sp}(l-j+1, j), \quad 0 \leqslant 2 j \leqslant l ; \\
\mathfrak{g}=\operatorname{su}(l+m, l): & \mathfrak{k}_{\varepsilon}=\operatorname{su}(l+m-j, j)+\operatorname{su}(l-j, j)+\operatorname{so}(2), \quad m \geqslant 1, \quad 0 \leqslant j \leqslant l ; \\
\mathfrak{g}=\operatorname{su}(l, l): & \mathfrak{k}_{\varepsilon}=\operatorname{su}(l-j, j)+\operatorname{su}(l-j, j)+\operatorname{so}(2), \quad 0 \leqslant 2 j \leqslant l ;
\end{array}
$$




$$
\begin{aligned}
& \mathfrak{g}=\operatorname{su}(l, l): \quad \mathfrak{k}_{\varepsilon}=\operatorname{sl}(l, \mathbb{C})+\mathbb{R} \\
& \mathfrak{g}=\operatorname{so}(2 l+1, \mathbb{C}): \quad \mathfrak{k}_{\varepsilon}=\operatorname{so}(2 l-2 j+1,2 j), \quad 0 \leqslant j \leqslant l ; \\
& \mathfrak{g}=\operatorname{so}(2 l, \mathbb{C}): \quad \mathfrak{k}_{\varepsilon}=\operatorname{so}(2 l-2 j, 2 j), \quad 0 \leqslant j \leqslant l ; \\
& \mathfrak{g}=\operatorname{so}(2 l, \mathbb{C}): \quad \mathfrak{k}_{\varepsilon}=\operatorname{so}^{*}(2 l) ; \\
& \mathfrak{g}=\operatorname{so}(l+m, l): \quad \mathfrak{k}_{\varepsilon}=\operatorname{so}(l-j+m, j)+\operatorname{so}(l-j, j), \quad m \geqslant 1, \quad 0 \leqslant j \leqslant l ; \\
& \mathfrak{g}=\operatorname{so}(l, l): \quad \mathfrak{k}_{\varepsilon}=\operatorname{so}(l-j, j)+\operatorname{so}(l-j, j), \quad 0 \leqslant 2 j \leqslant l ; \\
& \mathfrak{g}=\operatorname{so}(l, l): \quad \mathfrak{k}_{\varepsilon}=\operatorname{so}(l, \mathbb{C}) ; \\
& \mathfrak{g}=\mathrm{so}^{*}(4 l+2): \quad \mathfrak{k}_{\varepsilon}=\mathrm{u}(2 l-2 j+1,2 j), \quad 0 \leqslant 2 j \leqslant l ; \\
& \mathfrak{g}=\operatorname{so}^{*}(4 l): \quad \mathfrak{k}_{\varepsilon}=\mathrm{u}(2 l-2 j, 2 j), \quad 0 \leqslant 2 j \leqslant l ; \\
& \mathfrak{g}=\mathrm{so}^{*}(4 l): \quad \mathfrak{k}_{\varepsilon}=\mathrm{su}^{*}(2 l)+\mathrm{so}(1,1) \\
& \mathfrak{g}=\operatorname{sp}(l, \mathbb{C}): \quad \mathfrak{k}_{\varepsilon}=\operatorname{sp}(l-j, j), \quad 0 \leqslant 2 j \leqslant l ; \\
& \mathfrak{g}=\operatorname{sp}(l, \mathbb{C}): \quad \mathfrak{k}_{\varepsilon}=\operatorname{sp}(l, \mathbb{R}) ; \\
& \mathfrak{g}=\operatorname{sp}(l, \mathbb{R}): \quad \mathfrak{k}_{\varepsilon}=\mathrm{u}(l-j, j), \quad 0 \leqslant 2 j \leqslant l ; \\
& \mathfrak{g}=\operatorname{sp}(l, \mathbb{R}): \quad \mathfrak{k}_{\varepsilon}=\operatorname{gl}(l, \mathbb{R}) ; \\
& \mathfrak{g}=\operatorname{sp}(l+m, l): \quad \mathfrak{k}_{\varepsilon}=\operatorname{sp}(l+m-j, j)+\operatorname{sp}(l-j, j), \quad m \geqslant 1, \quad 0 \leqslant j \leqslant l ; \\
& \mathfrak{g}=\operatorname{sp}(l, l): \quad \mathfrak{k}_{\varepsilon}=\operatorname{sp}(l-j, j)+\operatorname{sp}(l-j, j), \quad 0 \leqslant 2 j \leqslant l ; \\
& \mathfrak{g}=\operatorname{sp}(l, l): \quad \mathfrak{k}_{\varepsilon}=\operatorname{sp}(l, \mathbb{C}) .
\end{aligned}
$$

A generalized Cartan decomposition of the Lie group $G$ with respect to a pair of subgroups $\left(K_{\varepsilon}, K\right)$ has a form

$$
G=K_{\varepsilon} A K
$$

(see [46]). If

$$
k_{1} h_{1} k_{1}^{\prime}=k_{2} h_{2} k_{2}^{\prime}, \quad k_{1}, k_{2} \in K_{\varepsilon}, \quad h_{1}, h_{2} \in A, \quad k_{1}^{\prime}, k_{2}^{\prime} \in K,
$$

then

$$
k_{1}^{\prime} k_{2}^{\prime-1}=k_{1}^{-1} k_{2} \in K \bigcap K_{\varepsilon}, \quad h_{1}=\left(k_{1}^{-1} k_{2}\right) h_{2}\left(k_{1}^{-1} k_{2}\right)^{-1} .
$$

\subsection{Generalized Cartan decomposition and $H$-coordinate systems on $G / K$}

The decomposition (12.19) does not give a unique decomposition $g=k h k^{\prime}$ of elements $g \in G$ into a product of elements of $K_{\varepsilon}, A$ and $K$. In order to have a unique decomposition we use the following procedure. Let $\mathfrak{a}_{\varepsilon}^{+}$denote the set of all elements $H \in \mathfrak{a}$ for which $\alpha(H)>0$ for each $\alpha \in \Sigma_{\varepsilon}^{+}$, where $\Sigma_{\varepsilon}^{+}$is the set of positive roots in $\Sigma_{\varepsilon}$. Let $A_{\varepsilon}^{+}=\exp \mathfrak{a}_{\varepsilon}^{+}$. We denote a set of representatives of cosets of $K_{\varepsilon} / M$ by $\left(K_{\varepsilon} / M\right)$. Then almost each element $g \in G$ decomposes uniquely as a product

$$
g=y h k, \quad y \in\left(K_{\varepsilon} / M\right), \quad h \in A_{\varepsilon}^{+}, \quad k \in K
$$

(see [46]). Note that the set $A_{\varepsilon}^{+}$does not coincide with the set $A^{+}$in (12.2). Moreover, the set $A_{\varepsilon}^{+}$is a union of the sets $w A^{+}$, where $w$ runs over a part of elements (including the unit element) of the Weyl group $W$ of the pair $(\mathfrak{g}, \mathfrak{a})$.

Taking into account the unique decomposition (12.20), we can state that the space $G / K$ is parametrized almost everywhere by elements from $\left(K_{\varepsilon} / M\right) A_{\varepsilon}^{+}$. A parametrization of $A_{\varepsilon}^{+}$can be obtained from (12.4), if we introduce necessary restrictions upon values of the parameters $t_{1}, t_{2}, \ldots, t_{l}$. 
Thus, a parametrization of the space $G / K$ (by using the generalized Cartan decomposition) is reduced to a parametrization of the space $K_{\varepsilon} / M$. Generally speaking, it is possible to choose many coordinate systems on $K_{\varepsilon} / M$. To each of such coordinate systems on $K_{\varepsilon} / M$ there corresponds a coordinate system on $G / K$. These coordinate systems on $G / K$ are called hyperbolic or H-systems.

A group $G$ may have many subgroups $K_{\varepsilon}$. To each of these subgroups there corresponds its coordinate systems on $G / K$.

Remark. The generalized Cartan decomposition (12.19) can be written in the form $G=K A K_{\varepsilon}$. This form of the decomposition can be used for parametrization of the pseudo-Riemannian symmetric space $G / K_{\varepsilon}$. This space is an analogue of the one-sheeted hyperboloid $S O_{0}(1,4) / S O_{0}(1,3)$ in the 5 -dimensional Minkowski space-time. The space $G / K_{\varepsilon}$ can be parametrized by elements from $(K / M) A_{\varepsilon}^{+}$; then the corresponding parametrization gives an analogue of the spherical coordinate system on $S O_{0}(1,4) / S O_{0}(1,3)$.

\subsection{Iwasawa decomposition and $S$-coordinate systems on $G / M N$}

Let $G=K A N$ be an Iwasawa decomposition of the group $G$. We represent elements of $K$ in the form of the product $k=\tilde{k} m, m \in M$, where $\tilde{k}$ is an element of $K$ representing a coset of $K / M$ containing the element $k$. The set of elements $\tilde{k}$ is denoted as $(K / M)$. Then the decomposition $G=K A N$ can be written as $G=(K / M) M A N=(K / M) A M N$. Therefore,

$$
(G / M N)=(K / M) A
$$

where $(G / M N)$ is a set of representatives of cosets in $G / M N$. The relation (12.4) gives a parametrization of elements $h \in A$ by the parameters $t_{1}, t_{2}, \ldots, t_{l}$. Thus, according to (12.21) a parametrization of the space $G / M N$ is reduced to a parametrization of the space $K / M$. A procedure of the latter parametrization was described above. In analogy with the parametrization of the upper sheet of the cone $C_{4}^{+}$(related to the group $G=S O_{0}(1,4)$ ), we call the coordinate system given by this parametrization of $G / M N$ spherical or the $S$-coordinate system.

Comparing the relations (12.3) and (12.21), we see that $S$-coordinate systems on $G / K$ and on $G / M N$ almost coincide. A difference consists in the fact that for the parametrization of $G / M N$ the whole subgroup $A$ is used, whereas for the parametrization of the space $G / K$ we used only its part $\overline{A^{+}}$.

In order to obtain a $G$-invariant measure on $G / M N$ we use the integral relation

$$
\int_{G} f(g) d g=\int_{K} \int_{A} \int_{N} f(k h n) e^{2 \rho(\log h)} d n d h d k,
$$

where $d g, d k, d h$ and $d n$ are invariant measures on $G, K, A$ and $N$, respectively. If $k=\tilde{k} m$, $m \in M, \tilde{k} \in(K / M)$, then

$$
\int_{K} f(k) d k=\int_{(K / M)} \int_{M} f(\tilde{k} m) d m d \tilde{k}
$$

where $d \tilde{k}$ is a $K$-invariant measure on $(K / M)$. It follows from $(12.22)$ that for $(G / K) \ni x=$ $\tilde{k} h \in(K / M) A$ we have

$$
\int_{G / K} f(x) d x=\int_{(K / M)} \int_{A} f(\tilde{k} h) e^{2 \rho(\log h)} d h d \tilde{k},
$$

where $d x$ is a $G$-invariant measure on $G / K$. 


\subsection{Gelfand-Naimark-Bruhat decomposition and $T$-coordinate systems on $G / M N$}

The decomposition of the Lie algebra $\mathfrak{g}$ into root subspaces is of the form

$$
\mathfrak{g}=\mathfrak{m}+\mathfrak{a}+\sum_{\alpha \in \Sigma} \mathfrak{g}_{\alpha}
$$

where $\Sigma$ is the set of restricted roots of the pair $(\mathfrak{g}, \mathfrak{a})$. We separate here the subspace $\overline{\mathfrak{n}}=\sum_{\alpha<0} \mathfrak{g}_{\alpha}$, where the summation is over all negative restricted roots of the pair $(\mathfrak{g}, \mathfrak{a})$. It is easy to see that $\overline{\mathfrak{n}}$ is a nilpotent subalgebra in $\mathfrak{g}$. Besides, $\overline{\mathfrak{n}}$ is obtained from $\mathfrak{n}$ by acting by the involutive Cartan automorphism $\theta: \theta \mathfrak{n}=\overline{\mathfrak{n}}$. We denote by $\bar{N}$ the analytical subgroup in $G$ with the Lie algebra $\overline{\mathfrak{n}}$. The set $\bar{N} A M N$ is everywhere dense in $G$ and almost each element $g \in G$ decomposes uniquely as a product $g=\tilde{n} h m n, \tilde{n} \in \bar{N}, h \in A, m \in M, n \in N$ (see [48]). This decomposition of $G$ is called the Gelfand-Naimark-Bruhat decomposition. We have

$$
(G / M N)=\bar{N} A \text {. }
$$

The equality here is understood in the sense that $\bar{N} A$ is everywhere dense in $(G / M N)$. The relation (12.4) gives a parametrization of the subgroup $A$ by numbers $t_{1}, t_{2}, \ldots, t_{l}$.

Thus, a parametrization of the space $G / M N$ is reduced to a parametrization of the subgroup $\bar{N}$. This subgroup can be parametrized by representing $\bar{N}$ in a form of a product of one parameter subgroups $\exp \mathfrak{g}_{\alpha}$. This parametrization is called translational (T-system of coordinates on $G / M N)$. The most interesting case is when the subgroup $\bar{N}$ is commutative. It is a case when $G=S O_{0}(1, n)$.

As we have seen, the $T$-coordinate system on $G / K$ is introduced by means of the subgroup $N A$ which differs from the subgroup $\bar{N} A$ in (12.24). However, it can be done that parametrizations of the corresponding subgroups $N$ and $\bar{N}$ (by means of which $T$-coordinate systems are introduced on $G / K$ and $G / M N$ ) will coincide with each other. For this aim we parametrize the set $G / K$ starting from the Iwasawa decomposition $G=\bar{N} A K$ of $G$. Then parametrizations of $G / K$ and $G / M N$ are fulfilled by means of parameters of the same set $\bar{N} A$.

A $G$-invariant measure $d x$ on $G / M N$ in the $T$-coordinate system is determined by the relation

$$
\int_{G / M N} f(x) d x=\int_{\bar{N}} \int_{A} f(\bar{n} h) e^{2 \rho(\log h)} d h d \bar{n},
$$

where $x$ represents the element $\bar{n} h \in \bar{N} A$, and $d \bar{n}$ and $d h$ are invariant measures on $\bar{N}$ and $A$, respectively.

\subsection{Gelfand-Naimark-Bruhat decomposition and $O$-coordinate systems on $G / M N$}

Let $G=\bar{N} A M N$ be the Gelfand-Naimark-Bruhat decomposition of the group $G$, where the equality is understood on a dense subspace in $G$. It can be represented in the form

$$
G=(\bar{N} M) A M N,
$$

where $\bar{N} M$ is a closed subgroup in $G$. This decomposition is not unique. Since elements of the subgroup $A$ commute with elements of $M$, then for obtaining a unique decomposition we have to take a set of representatives $y$ of cosets of $\bar{N} M / M$ instead of the subgroup $\bar{N} M$. We denote the set of these representative by $(\bar{N} M / M)$. It follows from (12.25) that $G=(\bar{N} M / M) A M N$, where the equality is understood on a dense subspace of $G$. Thus,

$$
(G / M N)=(\bar{N} M / M) A .
$$


The relation (12.4) gives a parametrization of the subgroup $A$ by numbers $t_{1}, t_{2}, \ldots, t_{l}$. The relation (12.26) reduces a parametrization of the space $G / M N$ to a parametrization of the space $(\bar{N} M / M)$. A parametrization of the cone $G / M N$, obtained in this way, is called orispherical $(O$ coordinate system). The $O$-coordinate system on the cone $C_{4}^{+}$for $G=S O_{0}(1,4)$ was considered in Section 8.

The most interesting case is when the subgroup $\bar{N}$ is commutative, that is, when $G=$ $S O_{0}(1, n)$. In this case, the subgroup $\bar{N} M$ is isomorphic to the group $I S O(n-1)$ (see [3], Chapter 9).

Comparing the parametrizations (12.14) and (12.26) of the spaces $G / K$ and $G / M N$, we see that they differ by appearing different nilpotent subgroups $N$ and $\bar{N}$. Replacing the Iwasawa decomposition $G=N A K$ by the decomposition $G=\bar{N} A K$ we obtain the relation

$$
(G / K)=(\bar{N} M / M) A
$$

instead of the relation (12.14). Then the spaces $G / K$ and $G / M N$ are parametrized by the same sets.

A $G$-invariant measure $d x$ on $G / M N$ in the $O$-coordinate system is determined by the integral relation

$$
\int_{G / M N} f(x) d x=\int_{(\bar{N} M / M)} \int_{A} f(y h) e^{2 \rho(\log h)} d h d y,
$$

where $d y$ is an $\bar{N} M$-invariant measure on $(\bar{N} M / M)$.

\subsection{Generalized Iwasawa decomposition}

The generalized Iwasawa decompositions are constructed by means of subgroups of $G$ used for construction of the generalized Cartan decomposition of $G$.

Let $M^{*}$ be a normalizer of the subgroup $A$ in $K$. We set $M_{\varepsilon}^{*}=K_{\varepsilon} \bigcap M^{*}$ and $W_{\varepsilon}=M_{\varepsilon}^{*} / M$. Then $W_{\varepsilon}$ is a symmetry group for the system of roots $\Sigma_{\varepsilon}$ (see [46]).

Elements of the quotient space $W_{\varepsilon} \backslash W$ can be represented by elements $w_{1} \equiv 1, w_{2}, \ldots, w_{r}$ of the subgroup $M^{*}$ which do not belong to $M_{\varepsilon}^{*}$. The integer $r$ is equal to the order of the quotient space $W_{\varepsilon} \backslash W$.

We create the sets $K_{\varepsilon} w_{i} A N, i=1,2, \ldots, r$. Then

(a) if $k w_{i} h n=k^{\prime} w_{j} h^{\prime} n^{\prime}$ with $k, k^{\prime} \in K_{\varepsilon}, h, h^{\prime} \in A, n, n^{\prime} \in N$, then $k=k^{\prime}, i=j, h=h^{\prime}$, $n=n^{\prime}$

(b) the mapping $(k, h, n) \rightarrow k w_{i} h n$ is an analytic diffeomorphism of the manifold $K_{\varepsilon} \times A \times N$ onto $K_{\varepsilon} w_{i} A N(i=1,2, \ldots, r)$;

(c) the set $\bigcup_{i=1}^{r} K_{\varepsilon} w_{i} A N$ is open and everywhere dense in $G$ (see [46]). Thus,

$$
G=\bigcup_{i=1}^{r} K_{\varepsilon} w_{i} A N .
$$

This equality is true on a dense manifold of $G$.

According to these assertions, almost each element $g \in G$ can be uniquely decomposed into a product

$$
g=k w_{i} h n, \quad k \in K_{\varepsilon}, \quad 1 \leqslant i \leqslant r, \quad h \in A, \quad n \in N .
$$

The equality (12.28) is called a generalized Iwasawa decomposition of the group $G$. This decomposition is written for those pseudo-Riemannian symmetric spaces $G / K_{\varepsilon}$, which are associated 
with the corresponding signatures $\varepsilon$ of the root system $\Sigma$. These symmetric spaces do not exhaust all pseudo-Riemannian symmetric spaces (see [45]). However, the decomposition (12.28) can be generalized to any pseudo-Riemannian symmetric space [49]. We consider the decomposition (12.28) only for spaces determined by signatures $\varepsilon$ of the root system $\Sigma$.

Integral relations for the usual Iwasawa decomposition of the group $G$ can be generalized to generalized Iwasawa decompositions. We have

$$
\int_{G} f(g) d g=\sum_{i=1}^{r} \int_{K_{\varepsilon}} \int_{A} \int_{N} f\left(k w_{i} h n\right) e^{2 \rho(\log h)} d n d h d k,
$$

where $f$ is a continuous function on $G$ with a compact support, and $d g, d k, d h, d n$ are invariant measures on $G, K_{\varepsilon}, A$ and $N$, respectively. This relation is true for an appropriate normalization of the measures. In another case, the right hand side must be multiplied by a constant.

\subsection{Generalized Iwasawa decomposition and $H$-coordinate systems on $G / M N$}

Let us write down the relation (12.28) in the form

$$
G=\bigcup_{i=1}^{r} K_{\varepsilon} w_{i} A M N
$$

where the equality is understood on a dense manifold in $G$. Since $A M=M A$ and $w_{i} M w_{i}^{-1}=M$, it can be represented as

$$
G=\bigcup_{i=1}^{r}\left(K_{\varepsilon} / M\right) w_{i} A M N
$$

where $\left(K_{\varepsilon} / M\right)$ is a set of representatives of cosets of the quotient space $K_{\varepsilon} / M$. The decomposition (12.30) obeys the following property: If

$$
y w_{i} h m n=y^{\prime} w_{j} h^{\prime} m^{\prime} n^{\prime}, \quad y, y^{\prime} \in\left(K_{\varepsilon} / M\right), \quad h, h^{\prime} \in A, \quad m, m^{\prime} \in M, \quad n, n^{\prime} \in N,
$$

then $y=y^{\prime}, i=j, h=h^{\prime}, m=m^{\prime}, n=n^{\prime}$. By other words, almost every element $g \in G$ can be uniquely decomposed as a product

$$
g=y w_{i} h m n, \quad y \in\left(K_{\varepsilon} / M\right), \quad 1 \leqslant i \leqslant r, \quad h \in A, \quad m \in M, \quad n \in N .
$$

We obtain from the decomposition (12.30) that

$$
(G / M N)=\bigcup_{i=1}^{r}\left(K_{\varepsilon} / M\right) w_{i} A
$$

where the equality is understood on a dense (in $G / M N$ ) set. The relation (12.4) gives a parametrization of the subgroup $A$ by the numbers $t_{1}, t_{2}, \ldots, t_{l}$. Therefore, the relation (12.31) reduces a parametrization of the space $G / M N$ to a parametrization of the quotient space $K_{\varepsilon} / M$. A parametrization of the cone $G / M N$, obtained in this way, is called hyperbolic. The corresponding coordinate system is called $H$-system of coordinates. Clearly, to different subgroups $K_{\varepsilon}$ there corresponds different coordinate systems on $G / M N$.

Remark. The generalized Iwasawa decomposition (12.28) can be written as

$$
G=\bigcup_{i=1}^{r} N A w_{i} K_{\varepsilon}
$$

This relation can be used for parametrization of the pseudo-Riemannian symmetric space $G / K_{\varepsilon}$ by elements of the set $N A \bigcup N A w_{2} \bigcup \cdots \cup N A w_{r}$. As a result, one obtains a $T$-coordinate system on $G / K_{\varepsilon}$. 


\section{Spectra of quasi-regular representations of $G$ on functions on $G / K$ and on $G / M N$}

\subsection{Quasi-regular representations of $G$}

We consider the Hilbert space $L^{2}(G / K)$ of functions on the hyperboloid $G / K$ and the Hilbert space $L^{2}(G / M N)$ of functions on the cone $G / M N$ with respect to $G$-invariant measures on $G / K$ and $G / M N$, respectively. The group $G$ acts on $G / K$ and $G / M N$ as a transitive motion group. To each element $g \in G$ there corresponds transformation $x \rightarrow g^{-1} x$ of points of $G / K$ and of points of $G / M N$. If some coordinate system is introduced on $G / K$ (on $G / M N$ ) then this transform can be written in terms of the corresponding coordinates.

The transform $x \rightarrow g^{-1} x$ on $G / K$ and on $G / M N$ gives a possibility to introduce unitary representations on $L^{2}(G / K)$ and on $L^{2}(G / M N)$. These representations are given by the formula

$$
\pi(g) f(x)=f\left(g^{-1} x\right), \quad x \in G / K \quad \text { or } \quad G / M N,
$$

and are called quasi-regular. The quasi-regular representation on $G / K$ will be denoted by $\pi^{H}$ and on $G / M N$ by $\pi^{C}$.

One of the main problems of our paper is to construct orthogonal bases on the Hilbert spaces $L^{2}(G / K)$ and $L^{2}(G / M N)$ for each coordinate system introduced above. To each coordinate system there corresponds a chain of subgroups of $G$ :

$$
\begin{aligned}
& S \text {-system: } G \supset K \supset \cdots, \\
& T \text {-system: } G \supset \bar{N} \supset \cdots, \\
& O \text {-system: } G \supset \bar{N} M \supset \cdots, \\
& H \text {-system: } G \supset K_{\varepsilon} \supset \cdots .
\end{aligned}
$$

There are several choices for each of these chains. An explicit form of other subgroups gives different varieties of a fixed type of coordinate systems. We do not consider them. As in the case of the group $G=S O_{0}(1,4)$, most interesting bases of $L^{2}(G / K)$ and $L^{2}(G / M N)$ are bases with separated variables. Irreducible representations of the group $G$ and of its subgroups are realized on parts of the corresponding bases. These basis functions are eigenfunctions of operators invariant with respect to the group $G$ and its subgroups associated with the corresponding coordinate system (see the case $G=S O_{0}(1,4)$ ). Eigenvalues of these operators (and also other indices if invariant operators have multiple eigenvalues) characterize basis functions. Therefore, we have to know a list of irreducible representations which are contained in the quasi-regular representations on $L^{2}(G / K)$ and on $L^{2}(G / M N)$ (these representations determine eigenvalues of the corresponding invariant operators). We also have to find decompositions of the irreducible representations of $G$, which are contained in the decomposition of the quasi-regular representations, into irreducible representations of the corresponding subgroups. These representations of subgroups determine a part of indices characterizing basis functions.

\subsection{Decomposition of quasi-regular representation $\pi^{H}$}

Let $\mathcal{F}$ be a space of real linear forms on the subalgebra $\mathfrak{a}$ of the Lie algebra $\mathfrak{g}$. The subset of $\mathcal{F}$, consisting of linear form $\lambda$ for which $\left(\lambda, \alpha_{i}\right) \geqslant 0$ for each simple restricted root $\alpha_{i}$ of the pair $(\mathfrak{g}, \mathfrak{a})$, is denoted by $\mathcal{F}^{+}$. Let $\pi_{\delta \lambda}$ be a principal nonunitary series representation of the group $G$ from section 10. We are interested in representations $\pi_{\delta \lambda}$ of class 1 with respect to the subgroup $K$ (that is, in representations $\pi_{\delta \lambda}$, which contain a trivial representation of $K$ ). A restriction $\left.\pi_{\delta \lambda}\right|_{K}$ of the representation $\pi_{\delta \lambda}$ upon the subgroup $K$ contains an irreducible representation $\omega$ of this subgroup if and only if a restriction of $\omega$ upon the subgroup $M$ contains the representation $\delta$ 
of $M$ (see [15]). Therefore, the representation $\pi_{\delta \lambda}$ is of class 1 with respect to $K$ if and only if $\delta$ is the trivial representation of the subgroup $M$. Such representations $\pi_{\delta \lambda}$ will be denoted by $\pi_{\lambda}$. The representations $\pi_{\lambda}$, for which $\lambda+\rho$ (where $\rho$ is the half-sum of positive restricted roots) are pure imaginary linear forms, are unitary (see, for example, [15]). They constitute class 1 representations of the principal unitary series of the group $G$. These representations are irreducible. In the class of the unitary representations $\pi_{\lambda}, \lambda \in \mathrm{i} \mathcal{F}-\rho$, equivalence relations exist. Namely, representations $\pi_{\lambda}$ and $\pi_{\lambda^{\prime}}$ are equivalent if and only if $\lambda^{\prime}=w(\lambda+\rho)-\rho$, $w \in W$. In order to obtain non-equivalent representations we have to restrict ourselves by the representations $\pi_{\lambda}, \lambda \in \mathrm{i} \mathcal{F}^{+}-\rho$.

Let $g \in G$ and $k \in K$. Then for the element $g^{-1} k$ we have a decomposition

$$
g^{-1} k=k_{g} h n, \quad k_{g} \in K, \quad h \in A, \quad n \in N
$$

(the Iwasawa decomposition). We represent the element $h$ in this decomposition in the form

$$
h=\exp H\left(g^{-1} k\right), \quad H\left(g^{-1} k\right) \in \mathfrak{a} .
$$

By means of this element we introduce a Fourier transform of functions on $G / K$. If $f \in$ $C_{c}^{\infty}(G / K)$, where $C_{c}^{\infty}(G / K)$ is the set of infinitely differentiable functions on $G / K$ with compact support, then $f$ can be considered as a function on $G$ which is constant on cosets from $G / K$. A Fourier transform of the function $f$ is a function on $K / M \times \mathcal{F}$ determined by

$$
\tilde{f}(\tilde{k}, \nu)=\int_{G} f(g) \exp \left\{(-\mathrm{i} \nu-\rho)\left[H\left(g^{-1} k\right)\right]\right\} d g, \quad \nu \in \mathcal{F},
$$

where $\tilde{k}$ is the coset in $K / M$ which contains the element $k \in K$. The function $\tilde{f}(\tilde{k}, \nu)$ can be represented as a function on $K$ constant on cosets of $K / M$. Let us prove that if $f(g)$ transforms under action of $G$ by quasi-regular representation $\pi^{H}$ of $G$, then $\tilde{f}(\tilde{k}, \nu)$ transforms under the representation $\pi_{\lambda} \equiv \pi_{-\mathrm{i} \nu-\rho}$ of the principal unitary series. Indeed, if $f(g)$ is replaced by $F(g):=\pi^{H}\left(g_{0}\right) f(g)=f\left(g_{0}^{-1} g\right)$, then the function (13.2) is replaced by

$$
\int_{G} f\left(g_{0}^{-1} g\right) \exp \left\{(-\mathrm{i} \nu-\rho)\left[H\left(g^{-1} k\right)\right]\right\} d g=\int_{G} f(g) \exp \left\{(-\mathrm{i} \nu-\rho)\left[H\left(g^{-1} g_{0}^{-1} k\right)\right]\right\} d g .
$$

Let us analyze the expression $H\left(g^{-1} g_{0}^{-1} k\right)$. Since

$$
g_{0}^{-1} k=k_{g_{0}} h n, \quad g^{-1} k_{g_{0}}=\left(k_{g_{0}}\right)_{g} h^{\prime} n^{\prime},
$$

then

$$
g^{-1} g_{0}^{-1} k=\left(k_{g_{0}}\right)_{g} h^{\prime} h n^{\prime \prime} n=\left(k_{g_{0}}\right)_{g} \exp \left[H\left(g^{-1} k_{g_{0}}\right)\right] \exp \left[H\left(g_{0}^{-1} k\right)\right] n^{\prime \prime} n .
$$

Therefore,

$$
H\left(g^{-1} g_{0}^{-1} k\right)=H\left(g^{-1} k_{g_{0}}\right)+H\left(g_{0}^{-1} k\right) .
$$

Then

$$
\exp \left\{(-\mathrm{i} \nu-\rho)\left[H\left(g^{-1} g_{0}^{-1} k\right)\right]\right\}=\exp \left\{(-\mathrm{i} \nu-\rho)\left[H\left(g^{-1} k_{g_{0}}\right)\right]\right\} \exp \left\{(-\mathrm{i} \nu-\rho)\left[H\left(g_{0}^{-1} k\right)\right]\right\} .
$$

Substituting this expression into (13.3), we obtain that under the transition

$$
f(g) \rightarrow \pi^{H}\left(g_{0}\right) f(g) \equiv f\left(g_{0}^{-1} g\right)
$$


the function $\tilde{f}(\tilde{k}, \nu)$ turns into the function

$$
\begin{gathered}
\exp \left\{(-\mathrm{i} \nu-\rho)\left[H\left(g_{0}^{-1} k\right)\right]\right\} \int_{G} f(g) \exp \left\{(-\mathrm{i} \nu-\rho)\left[H\left(g^{-1} k_{g_{0}}\right)\right]\right\} d g \\
=\exp \left\{(-\mathrm{i} \nu-\rho)\left[H\left(g_{0}^{-1} k\right)\right]\right\} \tilde{f}\left(\tilde{k}_{g_{0}}, \nu\right)=\pi_{-\mathrm{i} \nu-\rho}\left(g_{0}\right) \tilde{f}(\tilde{k}, \nu) .
\end{gathered}
$$

This proves our assertion.

The transform (13.2) at fixed $\nu$ is called a Poisson transform on $G / K$ (see [51]). The function $f(g)$ from $(13.2)$ can be restored by means of the function $\tilde{f}(\tilde{k}, \nu)$ as [51]

$$
f(g)=|W|^{-1} \int_{\mathcal{F}} \int_{K / M} \tilde{f}(\tilde{k}, \nu) \exp \left\{(\mathrm{i} \nu-\rho)\left[H\left(g^{-1} k\right)\right]\right\}|c(\nu)|^{-2} d \tilde{k} d \nu .
$$

Moreover, the Plancherel formula

$$
\int_{G / K}|f(\tilde{g})|^{2} d \tilde{g}=|W|^{-1} \int_{\mathcal{F}} \int_{K / M}|\tilde{f}(\tilde{k}, \nu)|^{2}|c(\nu)|^{-2} d \tilde{k} d \nu
$$

holds. In (13.4) and (13.5), $|W|$ means an order of the Weyl group $W, d \tilde{g}$ is a $G$-invariant measure on $G / K, d \tilde{k}$ is a $K$-invariant measure on $K / M$, and $d \nu$ is the Lebesgue measure on $\mathcal{F}$. The multiplier $c(\nu)$ in the Plancherel measure $|c(\nu)|^{-2} d \nu$ is determined by the formula

$$
c(\nu)=\int_{\bar{N}} \exp \{(-\mathrm{i} \nu-\rho)[H(\bar{n})]\} d \bar{n}=\frac{I(\mathrm{i} \nu)}{I(\rho)} .
$$

Here $H(\bar{n})$ is determined by the Iwasawa decomposition $\bar{n}=k \exp [H(\bar{n})] n, k \in K, n \in N$, $H(\bar{n}) \in \mathfrak{a}$, of the element $\bar{n} \in \bar{N}$, and $d \bar{n}$ is the normalized measure on $\bar{N}$ (see [15]). For $I(\nu)$ the following formula holds:

$$
I(\nu)=\prod_{\alpha \in \Sigma^{+}} B\left(\frac{m(\alpha)}{2}, \frac{m(\alpha / 2)}{4}+\frac{\langle\nu, \alpha\rangle}{\langle\alpha, \alpha\rangle}\right),
$$

where $\Sigma^{+}$is the set of positive restricted roots of the pair $(\mathfrak{g}, \mathfrak{a})$ (without taking into account multiplicities), $m(\alpha)$ is a multiplicity of the root $\alpha$, and $B(.,$.$) is the beta-function. The formulas$ (13.6) and (13.7) determine the Plancherel measure in (13.4) and (13.5). A proof of formulas (13.4)-(13.7) can be found in [15]. Note that formulas (13.2)-(13.5) demand certain normalization of the measures $d g, d \tilde{k}$ and $d \nu$. A violation of this normalization leads to multiplication of expressions by constants.

The function $\tilde{f}(\tilde{k}, \nu)$ on $K / M \times \mathcal{F}$ satisfies the additional condition (see [50])

$$
\int_{\mathcal{F}} \int_{K / M}|\tilde{f}(\tilde{k}, \nu)|^{2}|c(\nu)|^{-2} d \tilde{k} d \nu=\int_{\mathcal{F}} \int_{K / M}|\tilde{f}(\tilde{k}, w \nu)|^{2}|c(\nu)|^{-2} d \tilde{k} d \nu
$$

where $w$ is any element of the Weyl group $W$. Hence, the relation (13.5) can be written in the form

$$
\int_{G / K}|f(\tilde{g})|^{2} d \tilde{g}=\int_{\mathcal{F}^{+}} \int_{K / M}|\tilde{f}(\tilde{k}, \nu)|^{2}|c(\nu)|^{-2} d \tilde{k} d \nu .
$$

Formulas (13.2), (13.4) and (13.8) can be continued onto the spaces $L^{2}(G / K)$ and $L^{2}(K / M \times$ $\mathcal{F}_{+}$) (the latter space is taken with respect to the measure $|c(\nu)|^{-2} d \tilde{k} d \nu$ ). Then they give a decomposition of the quasi-regular representation $\pi^{H}$ of the group $G$ into irreducible unitary constituents. According to this decomposition we may state that the representation $\pi^{H}$ of $G$ 
decomposes into a direct integral of the unitary representations $\pi_{\lambda} \equiv \pi_{\mathrm{i} \nu-\rho}, \nu \in \mathcal{F}^{+}$, of $G$ and each of these representations appears in the decomposition only once:

$$
\pi^{H}=\int_{\mathcal{F}^{+}} \oplus \pi_{\mathrm{i} \nu-\rho}|c(\nu)|^{-2} d \nu
$$

In order to separate in $L^{2}(G / K)$ those spaces, on which irreducible representations $\pi_{\mathrm{i} \nu-\rho}$ are realized, we have to take by means of the integral transform (see formula (13.4))

$$
\left.\int_{K / M} \tilde{f}(\tilde{k}, \nu) \exp \left\{(\mathrm{i} \nu-\rho) \mid H\left(g^{-1} k\right)\right]\right\} d \tilde{k}
$$

an image in $L^{2}(G / K)$ of the space $L^{2}(K / M) \equiv L_{0}^{2}(K)$, on which $\pi_{\mathrm{i} \nu-\rho}$ is realized.

\subsection{Decomposition of quasi-regular representation $\pi^{C}$}

The problem of decomposition of the quasi-regular representation $\pi^{C}$ into irreducible representations of $G$ can be solved by means of the usual Fourier transform on the commutative group $A$. Let $C_{c}^{\infty}(G / M N)$ be the space of infinitely differentiable functions on $G / M N$ with a compact support. This space is everywhere dense in $L^{2}(G / M N)$. Functions $f \in C_{c}^{\infty}(G / M N)$ are considered as functions $f(\tilde{k}, h)$ on $(K / M) A$ (see subsection 11.7). For these functions the following transform can be constructed:

$$
\tilde{f}(\tilde{k}, \nu)=\int_{A} f(\tilde{k}, h) \exp \{(\mathrm{i} \nu+\rho)(\log h)\} d h, \quad \nu \in \mathcal{F} .
$$

It is easy to see that

$$
f(\tilde{k}, h)=\int_{\mathcal{F}} \tilde{f}(\tilde{k}, \nu) \exp \{(-\mathrm{i} \nu-\rho)(\log h)\} d \nu,
$$

and the Plancherel formula

$$
\int_{G / M N}|f(x)|^{2} d x \equiv \int_{K / M} d \tilde{k} \int_{A}|f(\tilde{k}, h)|^{2} e^{2 \rho(\log h)} d h=\int_{K / M} d \tilde{k} \int_{\mathcal{F}}|\tilde{f}(\tilde{k}, \nu)|^{2} d \nu
$$

holds.

Formulas (13.10)-(13.12) can be continued to the spaces $L^{2}(G / M N)$ and $L^{2}(K / M \times \mathcal{F})$. Using the formula (13.10) it is easy to show that if a function $f(\tilde{k}, h) \in L^{2}(G / M N)$ transforms under the representation $\pi^{C}$, then the function $\tilde{f}(\tilde{k}, \nu)$ at each fixed $\nu$ transforms under the irreducible unitary representation $\pi_{-\mathrm{i} \nu-\rho}$ of the group $G$. Since $f(\tilde{k}, h)$, as a function on $A$, does not satisfy additional conditions, then $\tilde{f}(\tilde{k}, \nu)$, as a function on $\mathcal{F}$, also does not satisfy any additional conditions. Formulas (13.10)-(13.12) give a decomposition of the representation $\pi^{C}$ of the group $G$ into irreducible representations $\pi_{\mathrm{i} \nu-\rho}$ of $G$ :

$$
\pi^{C}=\int_{\mathcal{F}} \oplus \pi_{\mathrm{i} \nu-\rho} d \nu
$$

The representations $\pi_{\mathrm{i} \nu-\rho}$ and $\pi_{w(\mathrm{i} \nu)-\rho}$ are unitary equivalent for any $w \in W$ and other equivalence relations in the set of the representations $\pi_{\mathrm{i} \nu-\rho}$ do not exist. Hence, the representation $\pi^{C}$ decomposes into a direct integral of all irreducible class 1 representations of the principal unitary series, and each of these representations is contained in the decomposition $|W|$ times, where $|W|$ is an order of the group $W$. 
In order to separate in $L^{2}(G / M N)$ a subspace of functions which are transformed under the irreducible representation $\pi_{\mathrm{i} \nu-\rho}$ it is necessary to make the integral transform

$$
\hat{f}(\tilde{k}, h ; \nu)=\int_{A} f\left(\tilde{k}, h h^{\prime}\right) e^{-(\mathrm{i} \nu-\rho)\left(\log h^{\prime}\right)} d h^{\prime} .
$$

(Speaking in a mathematically strict way, this space can be separated not in $L^{2}(G / M N)$, but in the space of infinitely differential functions on $G / M N$.) The functions (13.13) are homogeneous in $h$ of homogeneity degree $(\mathrm{i} \nu-\rho)$. It is easy to show that $\hat{f}(\tilde{k}, h ; \nu)$ are functions on the cone $G / M N \sim(K / M) A$ and are transformed under the representation $\pi_{\mathrm{i} \nu-\rho}$.

Let us consider other spectral problems, in particular, restrictions of representations $\pi_{\lambda}$ of the group $G$ onto the subgroups $K, \bar{N}, \bar{N} M, K_{\varepsilon}$, and decompositions of these restrictions into irreducible constituents.

\subsection{Restriction of representations $\pi_{\lambda}$ onto $K$}

A multiplicity of an irreducible unitary representation $\omega$ of the subgroup $K$ in $\pi_{\lambda} \downarrow_{K}$ coincides with a multiplicity of the unit (identity) representation of the subgroup $M$ in $\omega \downarrow_{M}$. This statement solves completely the problem of restriction of $\pi_{\lambda}$ onto $K$. It is known [50] that if the group $G$ is of real rank 1 (that is, $G$ coincides with one of the groups $S U(1, n), S O_{0}(1, n)$, $\left.S p(1, n), F_{4(-20)}\right)$, then the restriction $\left.\pi_{\lambda}\right|_{K}$ decomposes into a sum of all irreducible representations of $K$ which are of class 1 with respect to $M$ and their multiplicities in the decomposition are equal to 1.

\subsection{Restriction of representations $\pi_{\lambda}$ onto $\bar{N}$}

The representation $\pi_{\lambda}$ can be realized as follows. We take functions $f$ on $G$ satisfying the condition

$$
f(g)=f(x m n h)=\exp (-\lambda(\log h)) f(x),
$$

where $x$ are representatives of cosets of $G / M N A$. We construct a Hilbert space of such functions with the norm

$$
\|f\|^{2}=\int_{K}|f(k)|^{2} d k .
$$

The representation $\pi_{\lambda}$ is realized in this space by the formula

$$
\pi_{\lambda}\left(g_{0}\right) f(g)=f\left(g_{0}^{-1} g\right), \quad g_{0} \in G .
$$

Functions $f$ satisfying the condition (13.14) are determined uniquely by their values on representatives $x$. Therefore, $\pi_{\lambda}$ can be realized on functions $f(x)$. In order to give an action of the operators $\pi_{\lambda}(g)$ on functions $f(x)$ we take into account that $\pi_{\lambda}(g) f(x)=f\left(g^{-1} x\right)$ and decompose $g^{-1} x$ into a product of elements of $X, M, N$ and $A$ (where $X$ denotes the set of representatives $x)$ :

$$
g^{-1} x=x_{g} m^{\prime} n^{\prime} h_{g} .
$$

Since $f\left(g^{-1} x\right)=f\left(x_{g} m^{\prime} n^{\prime} h_{g}\right)=\exp \left(-\lambda\left(\log h_{g}\right)\right) f\left(x_{g}\right)$, then

$$
\pi_{\lambda}(g) f(x)=\exp \left(-\lambda\left(\log h_{g}\right)\right) f\left(x_{g}\right),
$$

where $h_{g}$ and $x_{g}$ are determined by (13.16). When we realize the representation $\pi_{\lambda}$ on the space of functions $f(x)$ it is desirable to have an expression for a norm in this space expressed by means of an integral over $X$. 
According to the Gelfand-Naimark-Bruhat decomposition of the group $G$, almost each element $g \in G$ can be decomposed in the form

$$
g=\bar{n} h m n=\bar{n} m n^{\prime} h, \quad \bar{n} \in \bar{N}, \quad m \in M, \quad n^{\prime} \in N, \quad h \in A .
$$

Hence, the subgroup $\bar{N}$ can be taken as a set $X$ of representatives $x$ of cosets of $G / M N A$. An action formula for operators $\pi_{\lambda}(g), g \in G$, upon functions $f(\bar{n})$ can be obtained in each concrete case by means of formulas (13.16) and (13.17). In this space we have the norm

$$
\|f\|^{2}=\int_{\bar{N}}|f(\bar{n})|^{2} d \bar{n}
$$

where $d \bar{n}$ is an invariant measure on $\bar{N}$, instead of the norm (13.15). The representation $\pi_{\mathrm{i} \nu-\rho}$, $\nu \in \mathcal{F}$, of the principal unitary series, realized on the Hilbert space $L^{2}(\bar{N})$ (with the norm (13.18)), is unitary and is unitary equivalent to the corresponding representation in the Hilbert space with the norm (13.15).

Restrict the representation $\pi_{\lambda}$ of the group $G$, realized on the space $L^{2}(\bar{N})$, upon the subgroup $\bar{N}$. It is easy to see that the operators $\pi_{\lambda}\left(\bar{n}_{0}\right), \bar{n}_{0} \in \bar{N}$, act on $L^{2}(\bar{N})$ as

$$
\pi_{\lambda}\left(\bar{n}_{0}\right) f(\bar{n})=f\left(\bar{n}_{0}^{-1} \bar{n}\right) .
$$

Thus, a restriction of the representation $\pi_{\lambda}$ upon the subgroup $\bar{N}$ is unitary equivalent to the regular representation of this subgroup. We conclude that a decomposition of regular representation of $\bar{N}$ gives a decomposition of the representation $\pi_{\lambda} \downarrow_{\bar{N}}$ into irreducible constituents.

In particular, if $G=S O_{0}(1, n)$, then $\bar{N}$ is a commutative group isomorphic to the group $T_{n-1}$ of real translations of dimension $n-1$ in $\mathbb{R}^{n-1}$. If $\left(x_{1}, x_{2}, \ldots, x_{n-1}\right) \in T_{n-1}$, then an irreducible unitary representation of $T_{n-1}$ can be written in the form

$$
x=\left(x_{1}, x_{2}, \ldots, x_{n-1}\right) \rightarrow e^{\mathrm{i}(x \cdot p)}, \quad p=\left(p_{1}, p_{2}, \ldots, p_{n-1}\right), \quad p_{i} \in \mathbb{R} .
$$

Here $x \cdot p=x_{1} p_{1}+x_{2} p_{2}+\cdots+x_{n-1} p_{n-1}$. The regular representation of the group $\bar{N}=T_{n-1}$ decomposes into a direct integral of all these irreducible unitary representations and each of these representations is contained in the decomposition once. The restriction of the representation $\pi_{\lambda}$ of $G$ upon the subgroup $\bar{N}=T_{n-1}$ decomposes into the same direct integral.

\subsection{Restriction of representations $\pi_{\lambda}$ onto $\bar{N} M$}

We represent the group $G$ in the form $G=(\bar{N} M / M) A M N$. Almost each element $g \in G$ decomposes uniquely as a product of elements of $(\bar{N} M / M), A, M$ and $N$. Therefore, the representation $\pi_{\lambda}$ of $G$ can be realized on the space of functions given on the set $(\bar{N} M / M)$. This set is homeomorphic to the subgroup $\bar{N}$. Hence, a norm on this space can be given by the formula (13.18). According to formulas (13.16) and (13.17), a restriction of the representation $\pi_{\lambda}$ upon $\bar{N} M$ acts upon the functions $f(x), x \in(\bar{N} M / M)$ by the formula

$$
\pi_{\lambda}(s) f(x)=f\left(s^{-1} x\right), \quad s \in \bar{N} M .
$$

Therefore, a restriction of the representation $\pi_{\lambda}$ of the group $G$ upon the subgroup $\bar{N} M$ is unitary equivalent to the quasi-regular representation of this subgroup, realized on the homogeneous space $\bar{N} M / M$.

In particular, if $G=S O_{0}(1, n)$, then $\bar{N} M \sim I S O(n-1)$. The quasi-regular representation of the group $I S O(n-1)$ on the space $L^{2}(I S O(n-1) / S O(n-1))$ decomposes into a direct integral of all unitary irreducible representations of $I S O(n-1)$ of class 1 with respect to the subgroup $M \sim S O(n-1)$ and each of these representations of $I S O(n-1)$ is contained in the decomposition only once (see, for example, [3]). These representations are given by a real positive number. 


\subsection{Restriction of representations $\pi_{\lambda}$ onto $K_{\varepsilon}$}

The generalized Iwasawa decomposition

$$
G=\bigcup_{i=1}^{r} K_{\varepsilon} w_{i} A N
$$

where the equality is understood on a dense subspace of $G$, can be written in the form

$$
G=\bigcup_{i=1}^{r}\left(K_{\varepsilon} / M\right) w_{i} M A N .
$$

Almost each element of $G$ decomposes as a product of elements of $\left(K_{\varepsilon} / M\right) w_{i}(i=1,2, \ldots, r)$, $M, A, N$. Then elements of the set

$$
X=\bigcup_{i=1}^{r}\left(K_{\varepsilon} / M\right) w_{i}
$$

can be taken as representatives of cosets of $G / M A N$. Thus, the representation $\pi_{\lambda}$ of the group $G$ can be realized on a space of functions given on $X$. The representation $\pi_{\mathrm{i} \nu-\rho}, \nu \in \mathcal{F}$, realized on the Hilbert space $L_{0}^{2}(K)$ of invariant (with respect to $M$ ) functions on $K$ with the norm (13.15), is unitary equivalent to the representation $\pi_{\mathrm{i} \nu-\rho}$ realized by formula (13.17) on the Hilbert space $L_{0}^{2}(X)$ of functions on the set (13.20) with the scalar product

$$
\left\langle f, f^{\prime}\right\rangle=\sum_{i=1}^{r} \int_{K_{\varepsilon}} f\left(k_{\varepsilon} w_{i}\right) \overline{f^{\prime}\left(k_{\varepsilon} w_{i}\right)} d k_{\varepsilon},
$$

where $d k_{\varepsilon}$ is an invariant measure on $K_{\varepsilon}$. It follows from (13.16) and (13.17) that a restriction of the representation $\pi_{\lambda}$ of $G$ upon the subgroup $K_{\varepsilon}$ acts on functions $f$ given on $X$ by the formula

$$
\pi_{\lambda}\left(k_{\varepsilon}^{0}\right) f\left(k_{\varepsilon} w_{i}\right)=f\left(\left(k_{\varepsilon}^{0}\right)^{-1} k_{\varepsilon} w_{i}\right), \quad k_{\varepsilon}^{0} \in K_{\varepsilon} .
$$

Thus, a restriction of the representation $\pi_{\lambda}$ of $G$ upon the subgroup $K_{\varepsilon}$ is unitary equivalent to the orthogonal sum of $r$ copies of the quasi-regular representation of $K_{\varepsilon}$ on the space of functions on $K_{\varepsilon} / M$. Since $M$ is a compact subgroup in $K_{\varepsilon}$, then the decomposition of the quasi-regular representation of $K_{\varepsilon}$ into irreducible constituents can be easily obtained from the decomposition into irreducible constituents of the regular representation of $K_{\varepsilon}$ (see [52]).

In particular, if $G=S O_{0}(1, n)$, then $M=S O(n-1)$. One of the possibilities for a subgroup $K_{\varepsilon}$ is $S O_{0}(1, n-1)$. We have $M \subset K_{\varepsilon}$ and $r=2$. A restriction of the representation $\pi_{\lambda}$ of the group $S O_{0}(1, n)$ upon the subgroup $K_{\varepsilon}=S O_{0}(1, n-1)$ is unitary equivalent to the orthogonal sum of two quasi-regular representations of $K_{\varepsilon}$ on the space of functions on $S O_{0}(1, n-1) / S O(n-1)$. This quasi-regular representation decomposes into a direct integral of all representations of class 1 (with respect to $S O(n-1)$ ) from the principal unitary series of $S O_{0}(1, n-1)$ and each of these representations is contained in the decomposition only once (see, for example, [3]).

\section{Expansion of functions on the cone $G / M N$}

In order to obtain basis functions on the cone $G / M N$ for each coordinate system, we can act as in the case $G=S O_{0}(1,4)$. For this, for each coordinate system it is necessary to find differential operators, invariant with respect to the group $G$ and its subgroups characterizing 
the corresponding coordinate systems, then to create a system of differential equations for their eigenfunctions, and to solve this system. As shown below, an expansion of functions on $G / M N$ is reduced to expansion of functions, given on homogeneous spaces of subgroups of smaller dimension. If the latter expansion is known, then we do not need to create a system of differential equations and to solve it. In other words, we shall construct basis functions by using the method of reduction of this problem to the problem for a smaller dimension (without using invariant differential operators). However, the basis functions, which will be obtained, are eigenfunctions of an appropriate system of invariant differential operators. Let us consider each coordinate system separately.

\subsection{Expansion for $S$-system}

Formulas (13.10)-(13.12) reduce an expansion of functions $f(x) \equiv f(\tilde{k}, h)$ on $G / M N$ to an expansion of functions, given on $K / M$. Indeed, under the integral in (13.11) we have a function $\tilde{f}(\tilde{k}, \nu)$, which for each fixed $\nu$ is transformed under irreducible representation $\pi_{\mathrm{i} \nu-\rho}$ of the group $G$ and belongs to $L_{0}^{2}(K) \equiv L^{2}(K / M)$. The function $\tilde{f}(\tilde{k}, \nu)$ can be expanded in matrix elements of irreducible representations of the compact subgroup $K$, invariant on the right with respect to the subgroup $M$. To obtain this expansion it is enough to apply the Peter-Weyl theorem.

Multiplying these representation matrix elements by the exponential functions $\exp [(-\mathrm{i} \nu-$ $\rho)(\log h)]$ and by $\exp [(\mathrm{i} \nu-\rho)(\log h)]($ at fixed $\nu)$ we obtain basis functions for irreducible spaces for the group $G$. Unification of these basis functions for all values of $\nu$ gives a (continuous) basis of the space $L^{2}(G / M N)$.

For example, if $G=S O_{0}(1, n)$, then $K=S O(n)$ and $M=S O(n-1)$. Expansion of functions given on the cone $G / M N$ is reduced by formula (13.11) to expansions on the sphere $S O(n) / S O(n-1)$. A basis for expansion of the latter functions consists of matrix elements of "null" column of irreducible representations of the subgroup $S O(n)$ with highest weights $(m, 0,0, \ldots, 0)$, that is of representations of class 1 with respect to the subgroup $S O(n-1)$ (see [3], Chapter 9). We denote these matrix elements by $D_{\alpha, 0}^{m}(k)$, where $\alpha$ enumerates basis elements of the space, where the irreducible representation with highest weight $(m, 0, \ldots, 0)$ acts. Then the collection of functions (see formula (13.11))

$$
D_{\alpha, 0}^{m}(\tilde{k}) \exp [(-\mathrm{i} \nu-\rho)(\log h)], \quad m=0,1,2, \ldots, \quad-\infty<\nu<\infty,
$$

with different $\alpha$ constitutes the basis of $L^{2}\left(S O_{0}(1, n) / M N\right)$, where $M N \sim I S O(n-1)$.

\subsection{Expansion for $T$-system}

Let us change decompositions (13.10)-(13.12). Namely, we consider functions $f \in C_{c}^{\infty}(G / M N)$ as functions $f(\bar{n}, h)$ on $\bar{N} A$. Then instead of relations (13.10)-(13.12) we have

$$
\begin{aligned}
& \tilde{f}(\bar{n}, \nu)=\int_{A} f(\bar{n}, h) e^{(\mathrm{i} \nu+\rho)(\log h)} d h, \\
& f(\bar{n}, h)=\int_{\mathcal{F}} \tilde{f}(\bar{n}, \nu) e^{(-\mathrm{i} \nu-\rho)(\log h)} d \nu \\
& \int_{G / M N}|f(x)|^{2} d x \equiv \int_{\bar{N}} d \bar{n} \int_{A}|f(\bar{n}, h)|^{2} e^{2 \rho(\log h)} d h=\int_{\bar{N}} d \bar{n} \int_{\mathcal{F}}|\tilde{f}(\bar{n}, \nu)|^{2} d \nu .
\end{aligned}
$$

Thus, anexpansion of functions $f \in C_{c}^{\infty}(G / M N)$ in basis functions is reduced to an expansion in basis functions, given on $\bar{N}$. For each fixed $\nu$, the functions $\tilde{f}(\bar{n}, \nu)$ are transformed under the left regular representation of the subgroup $\bar{N}$. They can be expanded in basis functions by means of decomposition of the regular representation of $\bar{N}$ into irreducible constituents. 
Let, for example, $G=S O_{0}(1, n)$. Then the subgroup $\bar{N}$ is isomorphic to $(n-1)$-dimensional group $T_{n-1}$ of translations. The exponential functions

$$
\begin{aligned}
& \operatorname{expi}(x \cdot p), \quad x=\left(x_{1}, x_{2}, \ldots, x_{n-1}\right) \in T_{n-1}, \\
& p=\left(p_{1}, p_{2}, \ldots, p_{n-1}\right), \quad-\infty<p_{i}<\infty,
\end{aligned}
$$

where $x \cdot p=x_{1} p_{1}+x_{2} p_{2}+\cdots+x_{n-1} p_{n-1}$, constitute a continuous basis for functions given on $\bar{N}$. Thus, the functions $f(x) \equiv f(\bar{n}, h)$ can be expanded in the functions

$$
\operatorname{expi}(x \cdot p) \exp \left[(\exp (-\mathrm{i} \nu-\rho)(\log h)], \quad-\infty<p_{i}<\infty, \quad-\infty<\nu<\infty .\right.
$$

Using formulas (14.1)-(14.3) and formulas for expansion of functions $f(\bar{n})$ in the basis (14.4), it is easy to write down formulas for expansions of functions $f(\bar{n}, h)$ in the basis (14.5) and the corresponding Plancherel formula.

\subsection{Expansion for $O$-system}

In this coordinate system the cone $G / M N$ is parametrized by means of the set $(\bar{N} M / M) A$ (see formula (12.26)). We consider functions $f \in C_{c}^{\infty}(G / M N)$ as functions $f(y, h)$ on $(\bar{N} M / M) \times A$. The expansions

$$
\begin{aligned}
& \tilde{f}(y, \nu)=\int_{A} f(y, h) e^{(\mathrm{i} \nu+\rho)(\log h)} d h \\
& f(y, h)=\int_{\mathcal{F}} \tilde{f}(y, \nu) e^{(-\mathrm{i} \nu-\rho)(\log h)} d \nu \\
& \int_{G / M N}|f(x)|^{2} d x=\int_{\bar{N} M / M} d y \int_{A}|f(y, h)|^{2} e^{2 \rho(\log h)} d h=\int_{\bar{N} M / M} d y \int_{\mathcal{F}}|\tilde{f}(y, \nu)|^{2} d \nu
\end{aligned}
$$

hold. As we see, expansion of functions $f \in C_{c}^{\infty}(G / M N)$ in basis functions is reduced to expansion of functions given on $\bar{N} M / M$. At each fixed $\nu$ the functions $\tilde{f}(y, \nu)$ are transformed under the quasi-regular representation of the subgroup $\bar{N} M$. By means of decomposition of this quasi-regular representation into irreducible representations of the subgroup $\bar{N} M$, the functions $\tilde{f}(y, \nu)$ can be expanded in matrix elements of irreducible representations of $\bar{N} M$ right invariant with respect to the subgroup $M$.

If $G=S O_{0}(1, n)$, then the subgroup $\bar{N} M$ is isomorphic to the group $I S O(n-1)$. The quasiregular representation of $I S O(n-1)$ on the space of functions given on the homogeneous space $I S O(n-1) / S O(n-1)$ decomposes into a direct integral of irreducible unitary representations of $I S O(n-1)$ which are of class 1 with respect to the subgroup $S O(n-1)$. Matrix elements of these representations of $I S O(n-1)$ in $S O(n-1)$-basis are expressed in terms of Bessel functions and are given in [3]. In expansion of functions given on $I S O(n-1) / S O(n-1)$ only matrix elements of the "null" column take part. If $D_{\alpha, 0}^{R}(r), r \in I S O(n-1), 0<R<\infty$, are these matrix elements, then the functions

$$
D_{\alpha, 0}^{R}(r) e^{(-\mathrm{i} \nu-\rho)(\log h)}, \quad 0<R<\infty, \quad-\infty<\nu<\infty,
$$

constitute a collection of basis functions on $S O_{0}(1, n) / S O(n)$ in the $O$-coordinate system.

\subsection{Expansion for $H$-system}

In this coordinate system, the cone $G / M N$ is parametrized by the set

$$
X=\bigcup_{i=1}^{r}\left(K_{\varepsilon} / M\right) w_{i}, \quad w_{i} \in W_{\varepsilon} \backslash W .
$$


We represent a function $f \in C_{c}^{\infty}(G / M N)$ as a function $f(y, h)$ on $X \times A$. Then the transforms

$$
\begin{aligned}
& \tilde{f}(y, \nu)=\int_{A} f(y, h) e^{(\mathrm{i} \nu+\rho)(\log h)} d h, \\
& f(y, h)=\int_{\mathcal{F}} \tilde{f}(y, \nu) e^{(-\mathrm{i} \nu-\rho)(\log h)} d \nu, \\
& \int_{G / M N}|f(x)|^{2} d x=\int_{X} d y \int_{A}|f(y, h)|^{2} e^{2 \rho(\log h)} d h=\int_{X} d y \int_{\mathcal{F}}|\tilde{f}(y, \nu)|^{2} d \nu
\end{aligned}
$$

hold. Thus, an expansion of functions $f \in C_{c}^{\infty}(G / M N)$ in basis functions in $H$-coordinate system is reduced to an expansion of functions given on $K_{\varepsilon} / M$. At each fixed $\nu$ and $w_{i}$ the functions $\tilde{f}(y, \nu)$ are transformed under the quasi-regular representation of the subgroup $K_{\varepsilon}$. By means of decomposition of this quasi-regular representation into irreducible constituents, the functions $\tilde{f}(y, \nu)$ can be expanded in matrix elements of irreducible representations of $K_{\varepsilon}$, invariant on the right with respect to the subgroup $M$. Moreover, matrix elements of representations of $K_{\varepsilon}$ can correspond to different chains of subgroups of $K_{\varepsilon}$. Each basis function is non-vanishing only on one set $\left(K_{\varepsilon} / M\right) w_{i}$.

If $G=S O_{0}(1, n)$, then the subgroup $S O_{0}(1, n-1)$, containing the subgroup $M=S O(n-1)$, can be taken as the subgroup $K_{\varepsilon}$. In this case $r=2$. The elements $w_{1} \equiv 1$ and $w_{2}$ correspond to the upper and lower parts of the hyperboloid $H^{n-1}=S O_{0}(1, n) / S O(n-1)$. Thus,

$$
X=\left(K_{\varepsilon} / M\right) \bigcup\left(K_{\varepsilon} / M\right) w .
$$

The problem of construction of the whole collection of basis functions on the cone $G / M N$ in this case is reduced to construction of basis functions for the space $L^{2}\left(H^{n-1}\right)$ of functions on the hyperboloid $S O_{0}(1, n-1) / S O(n-1)$. On this hyperboloid different coordinate systems can be chosen. A detailed consideration of this case see in [3], Chapters 9 and 10.

\section{Expansion of functions on the hyperboloid $G / K$}

The first way of construction of collections of basis functions on the hyperboloid $G / K$, corresponding to different coordinate systems, is to use reasoning described for the case when $G=S O_{0}(1,4)$ in Section 7 . For $S O_{0}(1,4)$, for each coordinate system we have found a complete system of differential operators, invariant with respect to the group $G$ and its subgroups, which characterize the corresponding coordinate system. Then we have constituted the corresponding system of differential equations and have solved it.

The second way for solving the problem of construction of collections of basis functions consists in using the relations (13.2)-(13.5). Since it is not possible to construct a system of invariant differential operators for the case of a generic group $G$, we use the second way. For each concrete group $G$, the collection of basis functions, which will be found, a priori is a solution

of the corresponding system of invariant differential equations. Let us consider each coordinate system separately.

\subsection{Expansion for $S$-system}

According to the relation (13.4), a function $f(\tilde{g}) \in C_{c}^{\infty}(G / K)$ can be represented as

$$
f(\tilde{g})=|W|^{-1} \int_{\mathcal{F}}\left[\int_{K / M} \tilde{f}(\tilde{k}, \nu) \exp \left(\left\{(\mathrm{i} \nu-\rho)\left[H\left(g^{-1} k\right)\right]\right\} d \tilde{k}\right]|c(\nu)|^{-2} d \nu .\right.
$$

Let us consider the intrinsic integral on the right-hand side of this relation. The function $\tilde{f}(\tilde{k}, \nu)$, as a function on $K$ (constant on cosets with respect to $M$ ), can be expanded in matrix elements 
$D_{\alpha \alpha^{\prime}}^{\omega}(k)$ of irreducible representations $\omega$ of the group $K$. Only the matrix elements, invariant on the right with respect to the subgroup $M$, enter to the expansion. This condition implies a certain conditions for $\alpha^{\prime}$. Thus, by the Peter-Weyl theorem we have

$$
\tilde{f}(\tilde{k}, \nu)=\sum_{\omega, \alpha, \alpha^{\prime}} A_{\alpha \alpha^{\prime}}^{\omega \nu} D_{\alpha \alpha^{\prime}}^{\omega}(\tilde{k})
$$

and

$$
\begin{aligned}
& A_{\alpha \alpha^{\prime}}^{\omega \nu}=\int_{K / M} \tilde{f}(\tilde{k}, \nu) \overline{D_{\alpha \alpha^{\prime}}^{\omega}(\tilde{k})} d \tilde{k}, \\
& \sum_{\omega, \alpha, \alpha^{\prime}}\left|A_{\alpha \alpha^{\prime}}^{\omega \nu}\right|^{2}=\int_{K / M}|\tilde{f}(\tilde{k}, \nu)|^{2} d \tilde{k} .
\end{aligned}
$$

We substitute the expression (15.2) for $\tilde{f}(\tilde{k}, \nu)$ into the intrinsic integral on the right-hand side of the relation (15.1) (that is, into the integral (13.9)) and permute summation and integration. As a result, we obtain

$$
\mathcal{I}(g) \equiv \sum_{\omega, \alpha, \alpha^{\prime}} A_{\alpha \alpha^{\prime}}^{\omega \nu} \int_{K / M} D_{\alpha \alpha^{\prime}}^{\omega}(\tilde{k}) \exp \left\{(\mathrm{i} \nu-\rho)\left[H\left(g^{-1} k\right)\right]\right\} d \tilde{k} .
$$

Since in formula (15.1) we are interested in functions $f$ given on $G / K$ we can suppose that $g=k^{\prime} h$, where $k^{\prime}$ are representatives of cosets in $K / M$ and $h \in A^{+}$. Thus, we put $g=k^{\prime} h$. Making in (15.5) the substitution $k \rightarrow k^{\prime} k$ and taking into account the relation $d \tilde{k}=d\left(k^{\prime} \tilde{k}\right)$, we find that

$$
\begin{aligned}
\mathcal{I}(g) \equiv \mathcal{I}\left(k^{\prime} h\right) & =\sum_{\omega, \alpha, \alpha^{\prime}} A_{\alpha \alpha^{\prime}}^{\omega \nu} \int_{K / M} D_{\alpha \alpha^{\prime}}^{\omega}\left(k^{\prime} \tilde{k}\right) \exp \left\{(\mathrm{i} \nu-\rho)\left[H\left(h^{-1} k\right)\right]\right\} d \tilde{k} \\
& =\sum_{\omega, \alpha, \alpha^{\prime}} \sum_{\alpha^{\prime \prime}} A_{\alpha \alpha^{\prime}}^{\omega \nu} D_{\alpha \alpha^{\prime \prime}}^{\omega}\left(k^{\prime}\right) \int_{K / M} D_{\alpha^{\prime \prime} \alpha^{\prime}}^{\omega}(\tilde{k}) \exp \left\{(\mathrm{i} \nu-\rho)\left[H\left(h^{-1} k\right)\right]\right\} d \tilde{k} .
\end{aligned}
$$

The integral

$$
\int_{K / M} D_{\alpha^{\prime \prime} \alpha^{\prime}}^{\omega}(\tilde{k}) \exp \left\{(\mathrm{i} \nu-\rho)\left[H\left(h^{-1} k\right)\right]\right\} d \tilde{k} \equiv \mathcal{D}_{\omega\left(\alpha^{\prime \prime} \alpha^{\prime}\right), 0}^{\nu}(h)
$$

is a matrix element of the "null" column of the operator $\pi_{\mathrm{i} \nu-\rho}(h)$ in the $K$-basis, and

$$
\mathcal{I}(g) \equiv \mathcal{I}\left(k^{\prime} h\right)=\sum_{\omega, \alpha, \alpha^{\prime}} A_{\alpha \alpha^{\prime}}^{\omega \nu}\left[\sum_{\alpha^{\prime \prime}} D_{\alpha \alpha^{\prime \prime}}^{\omega}\left(k^{\prime}\right) \mathcal{D}_{\omega\left(\alpha^{\prime \prime} \alpha^{\prime}\right), 0}^{\nu}(h)\right] .
$$

Substituting this expression for $\mathcal{I}(g)$ into (15.1) one obtains the relation

$$
f(\tilde{g}) \equiv f(\tilde{k} h)=|W|^{-1} \int_{\mathcal{F}}\left[\sum_{\omega, \alpha, \alpha^{\prime}} A_{\alpha \alpha^{\prime}}^{\omega \nu} F_{\alpha \alpha^{\prime}}^{\omega \nu}\left(k^{\prime}, h\right)\right]|c(\nu)|^{-2} d \nu,
$$

where

$$
F_{\alpha \alpha^{\prime}}^{\omega \nu}\left(k^{\prime}, h\right)=\sum_{\alpha^{\prime \prime}} D_{\alpha \alpha^{\prime \prime}}^{\omega}\left(k^{\prime}\right) \mathcal{D}_{\omega\left(\alpha^{\prime \prime} \alpha^{\prime}\right), 0}^{\nu}(h) .
$$


From (15.3) and (13.2) we find the transform inverse to the transform (15.7):

$$
\begin{aligned}
A_{\alpha \alpha^{\prime}}^{\omega \nu} & =\int_{K / M} d \tilde{k}^{\prime} \int_{G} d g f(g) \exp \left\{(-\mathrm{i} \nu-\rho)\left[H\left(g^{-1} k^{\prime}\right)\right]\right\} \overline{D_{\alpha \alpha^{\prime}}^{\omega}\left(\tilde{k}^{\prime}\right)} \\
& =\int_{G} f(g) \overline{F_{\alpha \alpha^{\prime}}^{\omega \nu}\left(k^{\prime}, h\right)} d g
\end{aligned}
$$

where $g=k h k^{\prime}, k, k^{\prime} \in K, h \in A$. It follows from (15.4) and (13.8) that the Plancherel formula

$$
\int_{G / K}|f(\tilde{g})|^{2} d \tilde{g}=\int_{\mathcal{F}+} \sum_{\omega, \alpha, \alpha^{\prime}}\left|A_{\alpha \alpha^{\prime}}^{\omega \nu}\right|^{2}|c(\nu)|^{-2} d \nu
$$

holds. Thus, functions (15.8) constitute a complete collection of basis functions for the hyperboloid $G / K$.

If $G=S O_{0}(1, n)$, then $K=S O(n)$ and $M=S O(n-1)$. Irreducible representations of $K$, which are of class 1 with respect to $S O(n-1)$, are given by one non-negative integer $m$, and the matrix elements $D_{\alpha \alpha^{\prime}}^{\omega}(\tilde{k})$ from (15.8) and (15.3) take the form $D_{\alpha, 0}^{m}(k), k \in K$. We suppose that these matrix elements are taken in the basis corresponding to the chain of subgroups

$$
S O(n) \supset M \equiv S O(n-1) \supset S O(n-2) \supset \cdots .
$$

Thus, functions (15.8) are products of matrix elements of "null" column of a representation of the subgroup $S O(n)$ and matrix elements of "null" column of the operator $\pi_{\mathrm{i} \nu-\rho}(h)$ :

$$
F_{\alpha, 0}^{m \nu}(\tilde{k}, h)=D_{\alpha, 0}^{m}(\tilde{k}) \mathcal{D}_{m, 0}^{\nu}(h)
$$

(see [3] for an explicit form of these functions).

\subsection{Expansion for $T$-system}

Let us write down the formulas (13.2)-(13.5) in such form that the representation $\pi_{\mathrm{i} \nu-\rho}$ is realized on functions given on $\bar{N}$ (not on functions, given on $K / M$ ). According to (13.16) and (13.17), the operators $\pi_{\mathrm{i} \nu-\rho}(g), g \in G$, act on the space $L^{2}(\bar{N})$ with the norm (13.18) by the formula

$$
\pi_{\lambda}(g) f(\bar{n})=\exp \left\{-\lambda\left[H^{\prime}\left(g^{-1} \bar{n}\right)\right]\right\} f\left(\bar{n}_{g}\right),
$$

where $H^{\prime}\left(g^{-1} \bar{n}\right)$ and $\bar{n}_{g}$ are determined by

$$
\begin{aligned}
& g^{-1} \bar{n}=\bar{n}_{g} m \exp \left[H^{\prime}\left(g^{-1} \bar{n}\right)\right] n, \\
& \bar{n}_{g} \in \bar{N}, \quad m \in M, \quad n \in N, \quad H^{\prime}\left(g^{-1} \bar{n}\right) \in \mathfrak{a} .
\end{aligned}
$$

Due to these formulas, instead of the transform (13.2) we have the transform

$$
\tilde{f}(\bar{n}, \nu)=\int_{G / K} f(\tilde{g}) \exp \left\{(-\mathrm{i} \nu-\rho)\left[H^{\prime}\left(g^{-1} \bar{n}\right)\right]\right\} d \tilde{g} .
$$

Integration over $G / K$ here coincides with integration on the right-hand side of (12.12), where $N$ is replaces by $\bar{N}$. If the functions $f(\tilde{g})$ are transformed under the quasi-regular representation

$\pi^{H}$ of the group $G$, then the functions $\tilde{f}(\bar{n}, \nu)$ are transformed under the representations $\pi_{-\mathrm{i} \nu-\rho}$ (by the formula (15.11)). Instead of formulas (13.4) and (13.8) we have

$$
f(\tilde{g})=|W|^{-1} \int_{\mathcal{F}} \int_{\bar{N}} \tilde{f}(\bar{n}, \nu) \exp \left\{(\mathrm{i} \nu-\rho)\left[H^{\prime}\left(g^{-1} \bar{n}\right)\right]\right\}|c(\nu)|^{-2} d \bar{n} d \nu,
$$




$$
\int_{G / K}|f(\tilde{g})|^{2} d g=\int_{\mathcal{F}^{+}} \int_{\bar{N}}|\tilde{f}(\bar{n}, \nu)|^{2}|c(\nu)|^{-2} d \bar{n} d \nu .
$$

Now we expand $\tilde{f}(\bar{n}, \nu)$, as a function on $\bar{N}$, in matrix elements of irreducible unitary representations of the group $\bar{N}$ :

$$
\tilde{f}(\bar{n}, \nu)=\int_{\hat{N}} \sum_{\alpha, \alpha^{\prime}} A_{\alpha \alpha^{\prime}}^{\omega \nu} D_{\alpha \alpha^{\prime}}^{\omega}(\bar{n}) d \mu(\omega),
$$

where $\hat{N}$ denotes a set of irreducible unitary representations $\omega$ of the group $\bar{N}$. The coefficients $A_{\alpha \alpha^{\prime}}^{\omega \nu}$ are determined by the formula

$$
A_{\alpha \alpha^{\prime}}^{\omega \nu}=\int_{\bar{N}} \tilde{f}(\bar{n}, \nu) \overline{D_{\alpha \alpha^{\prime}}^{\omega}(\bar{n})} d \bar{n}
$$

and the Plancherel formula

$$
\int_{\bar{N}}|\tilde{f}(\bar{n}, \nu)|^{2} d \bar{n}=\int_{\hat{N}} \sum_{\alpha, \alpha^{\prime}}\left|A_{\alpha \alpha^{\prime}}^{\omega \nu}\right|^{2} d \mu(\omega)
$$

holds.

We substitute the expression (15.15) for $\tilde{f}(\bar{n}, \nu)$ into the intrinsic integral (the integral over $\bar{N}$ ) of the right-hand side of (15.13). If permutation of the integrations is possible in the obtained expression (we denote this expression by $\mathcal{I}(g)$ ), then

$$
\mathcal{I}(g)=\int_{\hat{N}} \sum_{\alpha, \alpha^{\prime}} A_{\alpha \alpha^{\prime}}^{\omega \nu} d \mu(\omega) \int_{\bar{N}} D_{\alpha \alpha^{\prime}}^{\omega}(\bar{n}) \exp \left\{(\mathrm{i} \nu-\rho)\left[H^{\prime}\left(g^{-1} \bar{n}\right]\right\} d \bar{n} .\right.
$$

Taking into account the $T$-parametrization of $G$, we assume that elements $g$ have the form $\bar{n}^{\prime} h$, $\bar{n}^{\prime} \in \bar{N}, h \in A$. Therefore, replacing $\bar{n}$ by $\bar{n}^{\prime} \bar{n}$ we find

$$
\mathcal{I}(g) \equiv \mathcal{I}\left(\bar{n}^{\prime} h\right)=\int_{\hat{N}} \sum_{\alpha, \alpha^{\prime}} A_{\alpha \alpha^{\prime}}^{\omega \nu} d \mu(\omega) \sum_{\alpha^{\prime \prime}} D_{\alpha \alpha^{\prime \prime}}^{\omega}\left(\bar{n}^{\prime}\right) \int_{\bar{N}} D_{\alpha^{\prime \prime} \alpha^{\prime}}^{\omega}(\bar{n}) \exp \left\{(\mathrm{i} \nu-\rho)\left[H^{\prime}\left(h^{-1} \bar{n}\right]\right\} d \bar{n} .\right.
$$

If the integral

$$
\int_{\bar{N}} D_{\alpha^{\prime \prime} \alpha^{\prime}}^{\omega}(\bar{n}) \exp \left\{(\mathrm{i} \nu-\rho)\left[H^{\prime}\left(h^{-1} \bar{n}\right]\right\} d \bar{n}\right.
$$

exists, we denote it as $\mathcal{D}_{\omega\left(\alpha^{\prime \prime} \alpha^{\prime}\right)}^{\nu}(h)$. Then it follows from (15.13) that

$$
f(\tilde{g}) \equiv f\left(\bar{n}^{\prime} h\right)=|W|^{-1} \int_{\mathcal{F}} \int_{\hat{N}} \sum_{\alpha, \alpha^{\prime}} A_{\alpha \alpha^{\prime}}^{\omega \nu} F_{\alpha \alpha^{\prime}}^{\omega \nu}\left(\bar{n}^{\prime}, h\right) d \mu(\omega)|c(\nu)|^{-2} d \nu,
$$

where

$$
F_{\alpha \alpha^{\prime}}^{\omega \nu}\left(\bar{n}^{\prime}, h\right)=\sum_{\alpha^{\prime \prime}} D_{\alpha \alpha^{\prime \prime}}^{\omega}\left(\bar{n}^{\prime}\right) \mathcal{D}_{\omega\left(\alpha^{\prime \prime} \alpha^{\prime}\right)}^{\nu}(h)
$$

Using formulas (15.12) and (15.16) one obtains the inverse transform

$$
A_{\alpha \alpha^{\prime}}^{\omega \nu}=\int_{\bar{N}} d \bar{n} \int_{G / K} d \tilde{g} f(\tilde{g}) \exp \left\{(-\mathrm{i} \nu-\rho)\left[H^{\prime}\left(g^{-1} \bar{n}\right]\right\} \overline{D_{\alpha \alpha^{\prime}}^{\omega}(\bar{n})}\right.
$$

A Plancherel formula for these transforms follows from (15.14) and (15.17). Thus, the functions (15.18) are basis functions on $G / K$ in the $T$-coordinate system. 


\subsection{Expansion for $O$-system}

Let us write down the formulas (13.2)-(13.5) in such form that the representation $\pi_{\mathrm{i} \nu-\rho}$ is realized on functions given on $\bar{N} M / M$ (not on $K / M$ ). According to (13.16) and (13.17), the operators $\pi_{\mathrm{i} \nu-\rho}(g), g \in G$, act on the space $L^{2}(\bar{N} M / M)$ by the formula

$$
\pi_{\lambda}(g) f(x)=\exp \left\{-\lambda\left[H^{\prime \prime}\left(g^{-1} x\right)\right]\right\} f\left(x_{g}\right), \quad x \in(\bar{N} M / M),
$$

where $H^{\prime \prime}\left(g^{-1} x\right)$ and $x_{g}$ are determined by

$$
\begin{aligned}
& g^{-1} x=x_{g} m \exp \left[H^{\prime \prime}\left(g^{-1} x\right)\right] n, \\
& x_{g} \in(\bar{N} M / M), \quad m \in M, \quad n \in N, \quad H^{\prime \prime}\left(g^{-1} x\right) \in \mathfrak{a} .
\end{aligned}
$$

Due to these formulas, instead of the transform (13.2) we consider the transform

$$
\tilde{f}(x, \nu)=\int_{G / K} f(\tilde{g}) \exp \left\{(-\mathrm{i} \nu-\rho)\left[H^{\prime \prime}\left(g^{-1} x\right)\right]\right\} d \tilde{g} .
$$

Integration on $G / K$ here coincides with integration on the right-hand side of (12.15), where $N$ is replaces by $\bar{N}$. If the functions $f(\tilde{g})$ are transformed under the quasi-regular representation $\pi^{H}$ of the group $G$, then the functions $\tilde{f}(x, \nu)$ are transformed under the representation $\pi_{-\mathrm{i} \nu-\rho}$ (by the formula (15.20)). Instead of formulas (13.4) and (13.8) we have

$$
\begin{aligned}
& f(\tilde{g})=|W|^{-1} \int_{\mathcal{F}} \int_{\bar{N} M / M} \tilde{f}(x, \nu) \exp \left\{(\mathrm{i} \nu-\rho)\left[H^{\prime \prime}\left(g^{-1} x\right)\right]\right\}|c(\nu)|^{-2} d x d \nu \\
& \int_{G / K}|f(\tilde{g})|^{2} d \tilde{g}=\int_{\mathcal{F}^{+}} \int_{\bar{N} M / M}|\tilde{f}(x, \nu)|^{2}|c(\nu)|^{-2} d x d \nu .
\end{aligned}
$$

Now our reasoning is as in the previous case. We expand $\tilde{f}(x, \nu)$, as a function on $\bar{N} M$, constant on cosets with respect to the subgroup $M$, in matrix elements of irreducible unitary representations of the group $\bar{N} M$ invariant on the right with respect to the $\operatorname{subgroup} M$ :

$$
\tilde{f}(x, \nu)=\int_{N M_{0}} \sum_{\alpha, \alpha^{\prime}} A_{\alpha \alpha^{\prime}}^{\omega \nu} D_{\alpha \alpha^{\prime}}^{\omega}(x) d \mu(\omega),
$$

where $\hat{N M_{0}}$ denotes a set of nonequivalent irreducible unitary representations of the group $\bar{N} M$ of class 1 with respect to the subgroup $M$. Invariance of matrix elements $D_{\alpha \alpha^{\prime}}^{\omega}(x)$ on the right with respect to $M$ imposes some conditions on values of $\alpha^{\prime}$. The coefficients $A_{\alpha \alpha^{\prime}}^{\omega \nu}$ are determined by the formula

$$
A_{\alpha \alpha^{\prime}}^{\omega \nu}=\int_{\bar{N} M / M} \tilde{f}(x, \nu) \overline{D_{\alpha \alpha^{\prime}}^{\omega}(x)} d x
$$

and the Plancherel formula

$$
\int_{\bar{N} M / M}|\tilde{f}(x, \nu)|^{2} d x=\int_{N M_{0}} \sum_{\alpha, \alpha^{\prime}}\left|A_{\alpha \alpha^{\prime}}^{\omega \nu}\right|^{2} d \mu(\omega)
$$

holds.

We substitute the expression (15.24) for the function $\tilde{f}(x, \nu)$ into the intrinsic integral (the integral over $x$ ) of the right-hand side of (15.22). If permutation of the integrations is possible in the obtained expression (we denote this expression by $\mathcal{I}(g)$ ), the

$$
\mathcal{I}(g)=\int_{\hat{N} M_{0}} \sum_{\alpha, \alpha^{\prime}} A_{\alpha \alpha^{\prime}}^{\omega \nu} d \mu(\omega) \int_{\bar{N} M / M} D_{\alpha \alpha^{\prime}}^{\omega}(x) \exp \left\{(\mathrm{i} \nu-\rho)\left[H^{\prime \prime}\left(g^{-1} x\right]\right\} d x .\right.
$$


Due to the $O$-parametrization of $G$, elements $g$ have the form $y h$, where $y$ are representatives of cosets of $\bar{N} M / M$ and $h \in A$. Replacing $x$ by $y x$ and using the relation $d x=d(y x)$ we find

$$
\mathcal{I}(g) \equiv \mathcal{I}(y h)=\int_{N M_{0}} \sum_{\alpha, \alpha^{\prime}} A_{\alpha \alpha^{\prime}}^{\omega \nu} d \mu(\omega) \sum_{\alpha^{\prime \prime}} D_{\alpha \alpha^{\prime \prime}}^{\omega}(y) \int_{\bar{N} M / M} D_{\alpha^{\prime \prime} \alpha^{\prime}}^{\omega}(x) \exp \left\{(\mathrm{i} \nu-\rho)\left[H^{\prime \prime}\left(h^{-1} x\right)\right]\right\} d x .
$$

If the integral

$$
\int_{\bar{N} M / M} D_{\alpha^{\prime \prime} \alpha^{\prime}}^{\omega}(x) \exp \left\{(\mathrm{i} \nu-\rho)\left[H^{\prime \prime}\left(h^{-1} x\right)\right]\right\} d x \equiv \mathcal{D}_{\omega\left(\alpha^{\prime \prime} \alpha^{\prime}\right)}^{\nu}(h)
$$

exists, then it follows from (15.22) that

$$
f(\tilde{g})=|W|^{-1} \int_{\mathcal{F}} \int_{N M_{0}} \sum_{\alpha, \alpha^{\prime}} A_{\alpha \alpha^{\prime}}^{\omega \nu} F_{\alpha \alpha^{\prime}}^{\omega \nu}(y, h) d \mu(\omega)|c(\nu)|^{-2} d \nu
$$

where

$$
F_{\alpha \alpha^{\prime}}^{\omega \nu}(y, h)=\sum_{\alpha^{\prime \prime}} D_{\alpha \alpha^{\prime \prime}}^{\omega}(y) \mathcal{D}_{\omega\left(\alpha^{\prime \prime} \alpha^{\prime}\right)}^{\nu}(h) .
$$

Using formulas (15.21) and (15.25) one obtains the inverse transform

$$
A_{\alpha \alpha^{\prime}}^{\omega \nu}=\int_{\bar{N} M / M} d x \int_{G / K} d \tilde{g} f(\tilde{g}) \exp \left\{(-\mathrm{i} \nu-\rho)\left[H^{\prime \prime}\left(g^{-1} x\right]\right\} \overline{D_{\alpha \alpha^{\prime}}^{\omega}(x)} .\right.
$$

A Plancherel formula for these transforms follows from this formula and from (15.23). Thus, the functions (15.27) are basis functions on $G / K$ in the $O$-coordinate system.

\subsection{Expansion for $\boldsymbol{H}$-system}

Let us give to formulas (13.2)-(13.5) such form for which the representations $\pi_{\mathrm{i} \nu-\rho}$ are realized on functions on the set

$$
X=\bigcup_{i=1}^{r}\left(K_{\varepsilon} / M\right) w_{i}
$$

instead of functions given on $K / M$. According to (13.16) and (13.17) the operators $\pi_{\lambda}(g), g \in G$, act on the space $L^{2}(X)$ by the formula

$$
\pi_{\lambda}(g) f(x)=\exp \left\{-\lambda\left[H^{\prime \prime \prime}\left(g^{-1} x\right)\right]\right\} f\left(x_{g}\right), \quad x \in X,
$$

where $H^{\prime \prime \prime}$ and $x_{g}$ are determined by the relation

$$
g^{-1} x=x_{g} m \exp \left[H^{\prime \prime \prime}\left(g^{-1} x\right)\right] n, \quad x_{g} \in X, \quad m \in M, \quad n \in N, \quad H^{\prime \prime \prime}\left(g^{-1} x\right) \in \mathfrak{a} .
$$

Therefore, instead of the transform (13.2) we consider the transform

$$
\tilde{f}(x, \nu)=\int_{G / K} f(\tilde{g}) \exp \left\{(-\mathrm{i} \nu-\rho)\left[H^{\prime \prime \prime}\left(g^{-1} x\right)\right] d \tilde{g} .\right.
$$

If the function $f(\tilde{g})$ is transformed under the quasi-regular representation $\pi^{H}$, then the function $\tilde{f}(x, \nu)$ is transformed under the representation $\pi_{-\mathrm{i} \nu-\rho}$. Instead of formulas (13.4) and (13.8) we have

$$
f(\tilde{g})=|W|^{-1} \int_{\mathcal{F}} \int_{X} \tilde{f}(x, \nu) \exp \left\{(\mathrm{i} \nu-\rho)\left[H^{\prime \prime \prime}\left(g^{-1} x\right)\right]\right\}|c(\nu)|^{-2} d x d \nu
$$




$$
\begin{gathered}
\equiv|W|^{-1} \sum_{i=1}^{r} \int_{\mathcal{F}} \int_{K_{\varepsilon}} \tilde{f}\left(k_{\varepsilon} w_{i}, \nu\right) \exp \left\{(\mathrm{i} \nu-\rho)\left[H^{\prime \prime \prime}\left(g^{-1} k_{\varepsilon} w_{i}\right)\right]\right\}|c(\nu)|^{-2} d k_{\varepsilon} d \nu \\
\int_{G / K}|f(\tilde{g})|^{2} d \tilde{g}=\sum_{i=1}^{r} \int_{\mathcal{F}^{+}} \int_{K_{\varepsilon}}\left|\tilde{f}\left(k_{\varepsilon} w_{i}, \nu\right)\right|^{2}|c(\nu)|^{-2} d k_{\varepsilon} d \nu
\end{gathered}
$$

where $d k_{\varepsilon}$ is an invariant measure on $K_{\varepsilon}$.

For each fixed $\nu$ and $w_{i}$ we expand the function $\tilde{f}\left(k_{\varepsilon} w_{i}, \nu\right)$ (as a function on the group $K_{\varepsilon}$, constant on cosets with respect to $M$ ) in matrix elements of irreducible unitary representations $\omega$ of the group $K_{\varepsilon}$ of class 1 with respect to the subgroup $M$ :

$$
\tilde{f}\left(k_{\varepsilon} w_{i}, \nu\right)=\int_{\hat{K}_{\varepsilon}} \sum_{\alpha, \alpha^{\prime}} A_{\alpha, \alpha^{\prime}}^{\omega \nu i} D_{\alpha \alpha^{\prime}}^{\omega}\left(k_{\varepsilon}\right) d \mu(\omega),
$$

where $\hat{K}_{\varepsilon}$ means the set of irreducible unitary representations of $K_{\varepsilon}$. The coefficients $A_{\alpha \alpha^{\prime}}^{\omega \nu i}$ are determined by the formula

$$
A_{\alpha \alpha^{\prime}}^{\omega \nu i}=\int_{K_{\varepsilon}} \tilde{f}\left(k_{\varepsilon} w_{i}, \nu\right) \overline{D_{\alpha \alpha^{\prime}}^{\omega}\left(k_{\varepsilon}\right)} d k_{\varepsilon}
$$

The Plancherel formula

$$
\int_{K_{\varepsilon}}\left|\tilde{f}\left(k_{\varepsilon} w_{i}, \nu\right)\right|^{2} d k_{\varepsilon}=\int_{\hat{K}_{\varepsilon}} \sum_{\alpha, \alpha^{\prime}}\left|A_{\alpha \alpha^{\prime}}^{\omega \nu i}\right|^{2} d \mu(\omega)
$$

holds. We substitute the expression (15.31) for the function $\tilde{f}\left(k_{\varepsilon} w_{i}, \nu\right)$ into the intrinsic integral in (15.29) (we denote this integral by $\mathcal{I}(g)$ ). If permutation of the integrals is allowed in the resulting expression for $\mathcal{I}(g)$, then

$$
\mathcal{I}(g)=\int_{\hat{K}_{\varepsilon}} \sum_{\alpha, \alpha^{\prime}} A_{\alpha \alpha^{\prime}}^{\omega \nu i} d \mu(\omega) \int_{K_{\varepsilon}} D_{\alpha \alpha^{\prime}}^{\omega}\left(k_{\varepsilon}\right) \exp \left\{(\mathrm{i} \nu-\rho)\left[H^{\prime \prime \prime}\left(g^{-1} k_{\varepsilon} w_{i}\right)\right]\right\} d k_{\varepsilon} .
$$

Suppose that elements $g$ here have the form $k_{\varepsilon}^{\prime} w_{i} h, h \in A$. Making in (15.34) the substitution $k_{\varepsilon} \rightarrow k_{\varepsilon}^{\prime} k_{\varepsilon}$ and using the relation $d k_{\varepsilon}=d\left(k_{\varepsilon}^{\prime} k_{\varepsilon}\right)$, we find that

$$
\begin{aligned}
\mathcal{I}(g) \equiv \mathcal{I}\left(k_{\varepsilon}^{\prime} w_{j} h\right)= & \int_{\hat{K}_{\varepsilon}} \sum_{\alpha, \alpha^{\prime}} A_{\alpha \alpha^{\prime}}^{\omega \nu i} d \mu(\omega) \sum_{\alpha^{\prime \prime}} D_{\alpha \alpha^{\prime \prime}}^{\omega}\left(k_{\varepsilon}^{\prime}\right) \\
& \times \int_{K_{\varepsilon}} D_{\alpha^{\prime \prime} \alpha^{\prime}}^{\omega}\left(k_{\varepsilon}\right) \exp \left\{(\mathrm{i} \nu-\rho)\left[H^{\prime \prime \prime}\left(h^{-1} w_{j}^{-1} k_{\varepsilon} w_{i}\right)\right]\right\} d k_{\varepsilon} .
\end{aligned}
$$

If the latter integral on the right hand side exists, we denote it as $\mathcal{D}_{\omega\left(\alpha^{\prime \prime}, \alpha^{\prime}\right)}^{\nu i}\left(w_{j} h\right)$. Then the formula (15.29) gives

$$
f(\tilde{g}) \equiv f\left(k_{\varepsilon}^{\prime} w_{j} h\right)=|W|^{-1} \sum_{i=1}^{r} \int_{\mathcal{F}} \int_{\hat{K}_{\varepsilon}} \sum_{\alpha, \alpha^{\prime}} A_{\alpha \alpha^{\prime}}^{\omega \nu i} F_{\alpha \alpha^{\prime}}^{\omega \nu i}\left(k_{\varepsilon}^{\prime}, w_{j} h\right)|c(\nu)|^{-2} d \mu(\omega) d \nu,
$$

where

$$
F_{\alpha \alpha^{\prime}}^{\omega \nu i}\left(k_{\varepsilon}^{\prime}, w_{j} h\right)=\sum_{\alpha^{\prime \prime}} D_{\alpha \alpha^{\prime \prime}}^{\omega}\left(k_{\varepsilon}^{\prime}\right) \mathcal{D}_{\omega\left(\alpha^{\prime \prime}, \alpha^{\prime}\right)}^{\nu i}\left(w_{j} h\right) .
$$

The inverse transform is of the form

$$
A_{\alpha \alpha^{\prime}}^{\omega \nu i}=\int_{K_{\varepsilon}} d k_{\varepsilon} \int_{G / K} d \tilde{g} f(\tilde{g}) \exp \left\{(-\mathrm{i} \nu-\rho)\left[H^{\prime \prime \prime}\left(g^{-1} k_{\varepsilon} w_{i}\right)\right]\right\} \overline{D_{\alpha \alpha^{\prime}}^{\omega}\left(k_{\varepsilon}\right)} .
$$


The Plancherel formula for these transforms follows from (15.30) and (15.33). Thus, the functions (15.35) are basis functions on $G / K$ in the $H$-coordinate system.

In the expansions considered above representations of the subgroups $\bar{N}, \bar{N} M$ and $K_{\varepsilon}$ were considered in discrete bases. The corresponding expansions can be similarly obtained also for the case when these representations are considered in "continuous" bases.

\subsection{Expansion of $K$-invariant functions on $G / K$}

A function $f$ on $G / K$ is called $K$-invariant if $f(k \tilde{g})=f(\tilde{g}), \tilde{g} \in G / K$. Since elements of $G / K$ are determined by representatives $g=k h, k \in K, h \in A$, then $K$-invariant functions $f$ on $G / K$ can be considered as functions on $A$ :

$$
f(\tilde{g})=f(k h)=F(h) .
$$

If $M^{\prime}$ is a normalizer of the subgroup $A$ in $K$, then for $m \in M^{\prime}$ we have $m A m^{-1}=A$ and

$$
F\left(m h m^{-1}\right)=F(h) .
$$

In other words, the function $F$ is uniquely determined by values on $\overline{A^{+}}$.

We consider the Fourier transform (13.2) of $K$-invariant functions $f$ on $G / K$ :

$$
\tilde{f}(\tilde{k}, \nu)=\int_{G} f(g) \exp \left\{(-\mathrm{i} \nu-\rho)\left[H\left(g^{-1} k\right)\right]\right\} d g, \quad \nu \in \mathcal{F} .
$$

Let us show that $\tilde{f}(\tilde{k}, \nu)$ is independent of $\tilde{k}$. For $k_{0} \in K$ we have

$$
\tilde{f}\left(k_{0} \tilde{k}, \nu\right)=\int_{G} f(g) \exp \left\{(-\mathrm{i} \nu-\rho)\left[H\left(g^{-1} k_{0} k\right)\right]\right\} d g .
$$

Since $H\left(g^{-1} k_{0} k\right)=H\left(\left(k_{0}^{-1} g\right)^{-1} k\right)$ and $d\left(k_{0} g\right)=d g$, then

$$
\begin{aligned}
\tilde{f}\left(k_{0} \tilde{k}, \nu\right) & =\int_{G} f\left(k_{0} g\right) \exp \left\{(-\mathrm{i} \nu-\rho)\left[H\left(g^{-1} k\right)\right]\right\} d\left(k_{0} g\right) \\
& =\int_{G} f(g) \exp \left\{(-\mathrm{i} \nu-\rho)\left[H\left(g^{-1} k\right)\right]\right\} d g=\tilde{f}(\tilde{k}, \nu) .
\end{aligned}
$$

This means that $\tilde{f}(\tilde{k}, \nu)$ is independent of $\tilde{k}$, that is $\tilde{f}(\tilde{k}, \nu)=\tilde{F}(\nu)$.

Thus, for $K$-invariant functions $f$ on $G / K$ we have the expansion

$$
f(\tilde{g})=|W|^{-1} \int_{\mathcal{F}} \int_{K / M} \tilde{F}(\nu) \exp \left\{(\mathrm{i} \nu-\rho)\left[H\left(g^{-1} k\right)\right]\right\}|c(\nu)|^{-2} d \tilde{k} d \nu,
$$

where

$$
\tilde{F}(\nu)=\int_{G} f(\tilde{g}) \exp \left\{(-\mathrm{i} \nu-\rho)\left[H\left(g^{-1} k\right)\right]\right\} d g .
$$

The function

$$
\varphi_{\nu}(g)=\int_{K / M} \exp \left\{(\mathrm{i} \nu-\rho)\left[H\left(g^{-1} k\right)\right]\right\} d \tilde{k}
$$

is called a zonal spherical function of the group $G$ corresponding to the representation $\pi_{\mathrm{i} \nu-\rho}$. This function is in fact the matrix element

$$
d_{11}^{\mathrm{i} \nu-\rho}(g)=\left\langle 1, \pi_{\mathrm{i} \nu-\rho}(g) 1\right\rangle
$$


of the representation $\pi_{\mathrm{i} \nu-\rho}$ of the group $G$. It is easy to check that

$$
\varphi_{\nu}\left(k g k^{\prime}\right)=\varphi_{\nu}(g), \quad k, k^{\prime} \in K,
$$

that is, $\varphi_{\nu}(g)$ is a $K$-invariant function on $G / K$.

It follows from (15.36) and (15.38) that

$$
f(\tilde{g})=|W|^{-1} \int_{\mathcal{F}} \tilde{F}(\nu) \varphi_{\nu}(g)|c(\nu)|^{-2} d \nu .
$$

According to the equality (12.8), the measure $d g$ in (15.37) can be decomposed into the product of the measures on $K$ and $A$. Since $f(\tilde{g})=F(h)$, then applying formula (15.38) we have

$$
\tilde{F}(\nu)=c|W|^{-1} \int_{\mathfrak{a}} F(\exp H) \varphi_{\nu}(\exp H)\left|\prod_{\alpha>0} \sinh \alpha(H)\right| d H .
$$

Using the formula (12.8) again we obtain

$$
\tilde{F}(\nu)=\int_{G} f(\tilde{g}) \varphi_{\nu}(g) d g .
$$

The Plancherel formula (13.5) now takes the form

$$
\int_{G / K}|f(\tilde{g})|^{2} d g=|W|^{-1} \int_{\mathcal{F}}|\tilde{F}(\nu)|^{2}|c(\nu)|^{-2} d \nu .
$$

The transform (15.41) is called a spherical transform of $K$-invariant functions $f$ on $G / K$. The formula (15.40) gives an inverse transform, which is an expansion of the function $f$ in zonal spherical functions of the group $G$. The formula (15.42) shows that the spherical transform is isometric.

In the spherical transform only the zonal spherical functions $\varphi_{\nu}(g)$ of the representations $\pi_{\mathrm{i} \nu-\rho}$ from the principal unitary series participate. The formula (15.38) determines zonal spherical

functions for all representations $\pi_{\mathrm{i} \nu-\rho}$ of the principal nonunitary series, that is, for representations with arbitrary complex linear forms $\nu$ on $\mathfrak{a}$.

\section{References}

[1] Fushchych W.I., Nikitin A.G., Symmetries of equations of quantum mechanics, Allerton Press, New York, 1994.

[2] Sitenko G.A., Scattering theory, Springer, Berlin, 1985.

[3] Vilenkin N.Ja., Klimyk A.U., Group representation and special functions, Vol. II, Kluwer, Dordrecht, 1993.

[4] Nikiforov A.F., Suslov S.K., Uvarov V.B., Classical orthogonal polynomials of discrete variables, Nauka, Moscow, 1985 (in Russian).

[5] Smirnov Yu.F., Shitikov K.V., K-harmonic method and the model of shells, Sov. J. Part. Nucl. 8 (1977), 847-910.

[6] Filippov G.F., Ovcharenko V.I., Smirnov Yu.F., Microscopic theory of collective exitations of atomic nuclears, Naukova Dumka, Kiev, 1981 (in Russian).

[7] Vilenkin N.Ja., Klimyk A.U., Group representation and special functions, Vol. I, Kluwer, Dordrecht, 1991.

[8] Basu D., Wolf K.B., The unitary irreducible representations of $S L(2, R)$ in all subgroup reductions, J. Math. Phys. 23 (1982), 189-208.

[9] Vilenkin N.Ja., Smorodinsky Ja.A., Invariant expansion of relativistic amplitudes, Soviet Physics JETP 19 (1964), 1209-1218. 
[10] Winternitz P., Smorodinsky Yu.A., Sheftel M.R., Poincaré and Lorentz invariant expansions of relativistic amplitudes, Yadernaya Fizika 7 (1968), 1325-1338.

[11] Winternitz P., Two variable expansions based on the Lorentz and conformal groups, in Lectures in Theoretical Physics, Editors A.O. Barut and W.E. Brittin, Colorado University Press, Boulder, 1971.

[12] Kalnins E.G., Patera J., Sharp R.T., Winternitz P., Two variable Galilei-group expansion of nonrelativistic scattering amplitudes, Phys. Rev. D 8 (1973), 2552-2572.

[13] Winternitz P., Poincaré groups, its little subgroups and their applications in particle physics, Lectures at the Summer School on Group Representations and Quantum Theory, Dublin, 1969.

[14] Winternitz P., Lucac I., Smorodinsky Yu.A., Quantum numbers in the little groups of the Poincaré group, Yadernaya Fizika 7 (1968), 192-201.

[15] Warner G., Harmonic analysis on semisimple Lie groups, Vols. 1, 2, Springer, Berlin, 1972.

[16] Helgason S., Differential geometry, Lie groups, and symmetric spaces, Academic Press, New York, 1978.

[17] Klimyk A.U., Kachurik I.I., Computation methods in theory of group representations, Vyshcha Shkola, Kiev, 1986 (in Russian).

[18] Vilenkin N.Ja., Klimyk A.U., Group representation and special functions, Vol. III, Kluwer, Dordrecht, 1992.

[19] Vilenkin N.Ja., Klimyk A.U., Group representation and special functions. Recent advances, Kluwer, Dordrecht, 1995.

[20] Erdelyi A., Magnus W., Oberheittinger F., Tricomi F., Higher transcendental functions, Vol. I, McGraw-Hill, New York, 1953.

[21] Erdelyi A., Magnus W., Oberheittinger F., Tricomi F., Higher transcendental functions, Vol. II, McGrawHill, New York, 1954.

[22] Boyer C.P., Matrix elements for the most degenerate principal series of representations of $S_{0}(p, 1)$, J. Math. Phys. 12 (1971), 1599-1603.

[23] Varlamov V.V., Spherical functions on the de Sitter group, J. Phys. A: Math. Teor. 40 (2007), 163-201, math-ph/0604026.

[24] Gel'fand I.M., Graev M.I., Applications of the method of orispheres to spectral analysis of functions on real and imaginary Lobachevsky spaces, Trudy Moscow Math. Soc. 11 (1962), 243-308.

[25] Vilenkin N.Ja., Special functions and the theory of group representations, Amer. Math. Soc., Providence, RI, 1968.

[26] Thomas L.H., On unitary representations of the group of de Sitter space, Ann. Math. 51 (1941), 113-126.

[27] Newton T.D., A note on the representations of the de Sitter group, Ann. Math. 60 (1950), 730-733.

[28] Dixmier J., Representations integrable du groupe de De Sitter, Bull. Soc. Math. France 89 (1961), 9-41.

[29] Takahashi R., Sur les representations unitaire des groupes de Lorentz generalises, Bull. Soc. Math. France 91 (1963), 289-433.

[30] Hirai T., On irreducible representations of the Lorentz group of $n$-th order, Proc. Japan Acad. 38 (1962), $258-262$.

[31] Gurseiy F., Group theoretical concepts and methods in elementary particle physics, Gordon and Breach, New York, 1964.

[32] Fronsdal C., Elementary particles in a curved space, Rev. Mod. Phys. 37 (1965), 221-224.

[33] Malkin I.A., Man'ko V.I., Dynamical symmetries and coherent states of quantum systems, Nauka, Moscow, 1979 (in Russian).

[34] Barut A.O., Bohm A., Dynamical groups and mass formulas, Phys. Rev. B 139 (1965), 1107-1112.

[35] Kachuryk I.I., Invariant expansions of solutions of 5-dimensional Klein-Gordon equation, Ukrainian Phys. J. 21 (1976), 1853-1862.

[36] Hirai T., The Plancherel formula for the Lorentz group of $n$-th order, Proc. Japan Acad. 42 (1966), 323-326.

[37] Limic N., Niederle J., Raczka R., Continuous degenerate representations of noncompact rotation groups, J. Math. Phys. 7 (1966), 2026-2035.

[38] Maurin K., General eigenfunction expansions and unitary representations of topological groups, PWN, Warszawa, 1968.

[39] Gel'fand I.M., Graev M.I., Vilenkin N.Ja., Integral geometry and related problems in the theory of representations, Vol. 5, Generalized functions, Academic Press, New York, 1966. 
[40] Gradshtein I.S., Ryzhik I.M., Table of integrals, series and products, Academic Press, New York, 1980.

[41] Ablamowitz M., Stegun I.A. (Editors), Handbook on special functions, National Bureau of Standards, New York, 1964.

[42] Zhelobenko D.P., Compact Lie groups and their representations, Amer. Math. Soc., Providence, RI, 1973.

[43] Knapp A.W., Representation theory of semisimple Lie groups. An overview based on examples, Princeton Univ. Press, Princeton, NJ, 1986.

[44] Klimyk A.U., Representation matrix elements and Clebsch-Gordan coefficients, Naukova Dumka, Kiev, 1979 (in Russian).

[45] Berger M., Les espaces symmetriques non compacts, Ann. Ecole Norm. Sup. 74 (1957), 85-177.

[46] Oshima T., Sekiguchi J., Eigenspaces of invariant differential operators on an affine symmetric space, Invent. Math. 57 (1980), 1-81.

[47] Muracami S., Sur la classification des algebres de Lie reelles et simples, Osaka J. Math. 2 (1965), 291-307.

[48] Bruhat F., Sur les representations induites des groupes de Lie, Bull. Soc. Math. France 84 (1956), 97-207.

[49] Matsuki T., The orbits of affine symmetric spaces under the action of minimal parabolic subgroups, J. Math. Soc. Jap. 31 (1981), 331-357.

[50] Helgason S., A duality for symmetric spaces, with applications to group representations, Adv. Math. 5 (1970), 1-154.

[51] Helgason S., Geometric analysis on symmetric spaces, Amer. Math. Soc., Providence, RI, 1994.

[52] Harish-Chandra, Harmonic analysis on the real reductive groups, Ann. Math. 104 (1976), 117-201. 